\title{
Metamaterials Based High Resolution 2-D Microwave Imaging
}

A Dissertation
Presented to
the faculty of the School of Engineering and Applied Science
University of Virginia
in partial fulfillment
of the requirements for the degree
Doctor of Philosophy
by
Duncan A. McGillivray
May




\section{Approval Sheet}

The dissertation is submitted in partial fulfillment of the requirements for the degree of

Doctor of Philosophy

Duncan A. McGillivray

The dissertation has been read and approved by the examining committee:

Professor Mool C. Gupta, Advisor (ECE)

Professor Robert M. Weikle, Committee Chair (ECE)

Professor N. Scott Barker (ECE)

Professor Christopher Goyne (MAE)

Dr. Robin L. Cravey (NASA-Langley Research Center)

Accepted for the School of Engineering and Applied Science:

Professor James H. Aylor, Dean, School of Engineering and Applied Science May 2015 
To the crew of the "Verrry Convenient" 


\section{Abstract}

Metamaterials research is a relatively new field that has exhibited tremendous growth since its

first successful demonstration in 2000. It even attracted attention in mainstream media, due to the metamaterials promise of novel capabilities in electromagnetics research, such as cloaking, extremely compact antenna design, high spectral resolution remote sensing, non-ionizing radiation protection, and subwavelength resolution imaging. Unlike conventional materials, metamaterials derive their electromagnetic properties through resonant subwavelength sized elements. Through precise engineering of these elements, the electromagnetic properties of the metamaterials can be tailored. While advances have been made in demonstrating metamaterials throughout the electromagnetic spectrum, applications in sensor systems and devices are just beginning to follow. Of particular interest is the metamaterial lens, which has been shown to provide subwavelength resolution. By overcoming the diffraction limit, these lenses lend themselves to high resolution imaging systems.

For microwave imaging devices, resolution has been of concern due to their long wavelengths. Furthermore, the systems are diffraction limited (far-field imaging) or subject to very short stand-off distance requirements (near-field imaging). With microwave metamaterial lenses it is now possible to achieve imaging of subwavelength sized features at larger working distances. Microwave metamaterial lenses are well suited to imaging applications because long wavelengths give the ability to peer inside of materials, while subwavelength resolution will render small details. In addition, microwave imaging is of particular interest to non-destructive testing, as it is non-ionizing, non-contact, and cost effective. This type 
of imaging system would lend itself to applications in medicine, automotive, aerospace, and security industries.

This dissertation presents the modeling, design, characterization, and validation of metamaterial lenses for high resolution 2-D microwave imaging. A 2-D metamaterial lens based on split ring resonator and rod constituent elements, optimized for $16.65 \mathrm{GHz}$, has been fabricated for the imaging application, and its imaging properties have been evaluated in a reflection mode configuration. Furthermore, the metamaterial lens capability for 2-D microwave imaging to detect hidden objects has been demonstrated. It was found that MTM lenses were able to image hidden objects at subwavelength resolution of $0.66 \lambda$ and sensitivity of $0.12 \lambda$ at a stand-off distance of $1.44 \lambda$.

This imaging system was the first of its kind to provide for 2-D microwave images using MTM lenses in reflection mode. Furthermore, the MTM lenses were found to be highly sensitive to the incident polarization, leading to unwanted rotation of the polarization state of transmitted MW. The polarization rotation has been related to the asymmetric unit cell design of typical MTM lenses. The effect of the polarization rotation in MTM lenses on image performance is the first to be discussed in literature. The current state of MTM based imaging system has been expanded upon by using a multi-detector array. This decreased image acquisition time by a factor of 5 while giving the potential of increasing the contrast of the image. The overall system provided for similar image quality as a near-field measurement at much greater stand-off distance than the near-field. This study highlights the capabilities and limitations of the metamaterial lens for imaging systems. 


\section{Acknowledgments}

I would like to thank my advisor Professor Mool C. Gupta, for his countless advice in research, support in navigating through academia, and continually guiding me in a forward direction. Moreover I would like to express my sincerest gratitude for providing me with the tremendous opportunity to study under the Langley Professor Program at NASA Langley Research Center, as this provided me with a one-of-a-kind experience to develop myself as a research engineer.

I would like to thank the NASA Langley Research Center, and the National Institute of Aerospace (NIA) for the Langley Professor Program. Furthermore, I would like to thank the Electromagnetics and Sensors Branch (ESB) for hosting me throughout my Ph.D. program. In particular, I would like to thank Dr. Robin Cravey and Kenneth Dudley for continually supporting and advising me in this research and making me feel at home at ESB. I owe my sincere gratitude to Robin and Ken, for their support, encouragement, and teaching me the tricks of the trade in experimental and computational electromagnetics.

I would also like to thank the ESB leadership Charmaine Franck, Jay Ely, Erik Vedeler, Sandra Koppen, and Delores Williams for their support and work towards having me as a student at ESB.

I owe much to my parents, friends and family for their constant encouragement and support. 


\section{Contents}

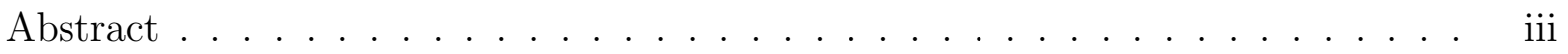

Acknowledgments ........................

List of Tables . . . . . . . . . . . . . . . . . . v viii

List of Figures . . . . . . . . . . . . . . . . . ix

List of Symbols and Abbreviations . . . . . . . . . . . . . . xi

1 Introduction and Literature Review 1

1.1 Metamaterials . . . . . . . . . . . . . . . . . . . . . . 1

1.2 Microwave Imaging . . . . . . . . . . . . . . . . . . . . . 3

1.3 Modeling . . . . . . . . . . . . . . . . . . . . 5

1.4 Problem Statement . . . . . . . . . . . . . . . . . . . . 5

1.5 Objectives and Research Goals . . . . . . . . . . . . . . . . 6

$\begin{array}{lll}2 & \text { Theoretical Background } & 7\end{array}$

2.1 Metamaterial Constituent Elements . . . . . . . . . . . . . . . . 8

2.1 .1 Rod elements and $-\epsilon_{e f f} \ldots \ldots \ldots \ldots \ldots$

2.1.2 Split Ring Resonators and $-\mu_{\text {eff }} \ldots \ldots \ldots \ldots$

2.1.3 Polarization Excitation . . . . . . . . . . . . . . . . . . . 12

2.2 Metamaterials Imaging . . . . . . . . . . . . . . . . . . . . . . . . . 13

2.2 .1 Focusing . . . . . . . . . . . . . . . . . . . . . 13

2.2.2 Evanescent Wave Enhancement . . . . . . . . . . . . . . . . . 15

2.3 Fundamental Limits of Imaging . . . . . . . . . . . . . . . . . . 17

2.3 .1 Far-Field Imaging . . . . . . . . . . . . . . . . . . . . 17

2.3 .2 Near-Field Imaging . . . . . . . . . . . . . . . . . . . . . . . . 18

2.3 .3 Metamaterial Lens . . . . . . . . . . . . . . . . . . . 20

3 Metamaterial Lens Modeling, Design, Fabrication, and Characterization 22

3.1 Modeling . . . . . . . . . . . . . . . . . . . . . . . . 22

3.1.1 Modeling of Electromagnetic Properties . . . . . . . . . . . . . 23

3.1 .2 Modeling of Object Imaging . . . . . . . . . . . . . . . . . 24

3.1 .3 Design of Antenna . . . . . . . . . . . . . . . . . . . . 26

3.2 Design and Fabrication . . . . . . . . . . . . . . . . . . . . . 27

3.2 .1 Metamaterial Lens . . . . . . . . . . . . . . . . . . . 27

3.2 .2 Antenna and Detectors . . . . . . . . . . . . . . . . . . . . . . 28

3.2 .3 Samples for Imaging $\ldots \ldots \ldots$

3.3 Characterization of Metamaterial Lens Properties . . . . . . . . . . . . 32 
3.4 Imaging Experimental Setup . . . . . . . . . . . . . . . . . . . . . . . . 34

3.4 .1 Transmission Mode Imaging . . . . . . . . . . . . . . . . . . . . 34

3.4 .2 Reflection Mode Imaging . . . . . . . . . . . . . . . . . . . . . . . 36

3.4 .3 Multi-Point Array Imaging . . . . . . . . . . . . . . . . 38

4 Metamaterial Lens Electromagnetic Properties Results and Discussion 41

4.1 Transmission and Reflection . . . . . . . . . . . . . . . . . . . 41

4.2 Index of Refraction . . . . . . . . . . . . . . . . . . . . . . . . . . . . . . . . . . . 48

4.3 Polarization Rotation by the Metamaterial Lens . . . . . . . . . . . . 53

$5 \quad$ Imaging Study Results and Discussion $\quad 57$

5.1 Transmission Mode Imaging . . . . . . . . . . . . . . . . . . . . 57

5.1 .1 Low Frequency Lens . . . . . . . . . . . . . . . . . 57

5.1 .2 High Frequency Lens . . . . . . . . . . . . . . . . . . 62

5.2 Reflection Mode Imaging - Single Detector . . . . . . . . . . . . . . . . . 68

5.2.1 Single Detector High Frequency Lens . . . . . . . . . . . . . . . 68

5.2 .2 Hidden Object Imaging . . . . . . . . . . . . . . . . . . . . . 75

5.2.3 Effect of Polarization Rotation on Imaging . . . . . . . . . . . . . . . 82

5.3 Reflection Mode Imaging using Multi-Detectors . . . . . . . . . . . . . . . . 84

5.4 Discussion . . . . . . . . . . . . . . . . . . . . . . . . . . . 92

6 Conclusions and Future Work $\quad 95$

$\begin{array}{ll}\text { List of Publications and Presentations } & 99\end{array}$

$\begin{array}{ll}\text { Bibliography } & 100\end{array}$

A Calibration for Free-Space Microwave Materials Characterization 106

B Detector Calibration $\quad 115$ 


\section{List of Tables}

3.1 Metamaterial design: feature sizes and substrate material . . . . . . . . . . . 29

3.2 Fabricated samples: feature sizes . . . . . . . . . . . . . . 31

4.1 Lens characterization: electromagnetic properties . . . . . . . . . . . . 52

5.1 Reflection mode: single detector aperture results . . . . . . . . . . . . . . . 69

5.2 Reflection mode: single detector resolution and sensitivity $\ldots \ldots \ldots 77$

A.1 Appendix: reference standard definition . . . . . . . . . . . . . . . 108 


\section{List of Figures}

1.1 MTM lens based microwave imaging concept . . . . . . . . . . . . . . . . 4

2.1 Basic rod elements . . . . . . . . . . . . . . . . . . . . . 9

2.2 Basic split ring resonator . . . . . . . . . . . . . . . . . . . . 12

2.3 Ray transmission through negative index lens and ordinary thin lens . . . . . 14

2.4 Fraunhofer diffraction patterns from two circular apertures . . . . . . . . . . 19

3.1 Unit cell setup for modeling . . . . . . . . . . . . . . . . . . . . . . 23

3.2 Modeling setup for transmission and reflection mode imaging . . . . . . . . . 25

3.3 Modeled antennas for the multi-detector array . . . . . . . . . . . . 26

3.4 Feature sizes and arrangement of the MTM constituent elements . . . . . . 27

3.5 MTM unit cell configurations . . . . . . . . . . . . . . . . . . 28

3.6 Detector circuit design . . . . . . . . . . . . . . . . . . . . . . . . . . . . . . . . . . . .

3.7 Schematic of dual apertures and dual hole samples . . . . . . . . . . . . . . 31

3.8 Exp. setup for EM properties and polarization rotation of the MTM lens . . 33

3.9 Exp. setup for transmission mode imaging . . . . . . . . . . . . . . 35

3.10 Exp. setup for reflection mode imaging . . . . . . . . . . . . . . . 37

3.11 Fabricated multi-detector array . . . . . . . . . . . . . . . . . . 40

4.1 Transmission and reflection of the LFL, HFL, and Teflon . . . . . . . . . . 42

4.2 Reflection phase information of the LFL, HFL, and Teflon . . . . . . . . 43

4.3 Transmitted phase for LFL, HFL, and Teflon . . . . . . . . . . . . . . . . 44

4.4 Effect of tolerances onto modeled transmission for 2-D LFL . . . . . . . . . . 46

4.5 Effect of tolerances onto modeled transmission for 2-D HFL. . . . . . . . . . 47

4.6 Retrieved $n_{\text {eff }}$ for 2-D LFL, 2-D HFL, and Teflon . . . . . . . . . . . 51

4.7 Incident polarization effect on transmission of MTM lens configurations . . . 54

4.8 Polarization rotation for 2-D LFL, 1-D LFL, and No-Lens . . . . . . . . 55

5.1 2-D LFL trans. mode imaging characteristics . . . . . . . . . . . . . . 58

5.2 1-D LFL trans. mode imaging characteristics . . . . . . . . . . . . . . . 61

5.3 HFL trans. mode imaging characteristics for various aperture stand-off . . . 63

5.4 HFL trans. mode imaging comparison modeling, experiment, and near-field . 64

5.5 HFL trans. mode imaging of dual aperture . . . . . . . . . . . . . . . 65

5.6 HFL trans. mode imaging characteristics of dual apertures . . . . . . . . . 66

5.7 HFL refl. mode imaging of single apertures . . . . . . . . . . . . . . . . 71

5.8 HFL refl. mode imaging characteristics for single apertures . . . . . . . . . . 72 
5.9 HFL refl. mode imaging of dual apertures . . . . . . . . . . . . . . 73

5.10 HFL refl. mode resolution of dual apertures . . . . . . . . . . . . . 74

5.11 HFL imaging of hidden dual apertures . . . . . . . . . . . . . . 75

5.12 2-D imaging of hidden dual holes . . . . . . . . . . . . . . 76

5.13 Referenced 2-D images of dual blind holes with various separation . . . . . 78

5.14 2-D image of a hidden single hole . . . . . . . . . . . . . . . . . . 79

5.15 Referenced 2-D images of various sized hidden holes. . . . . . . . . . . 80

5.16 2-D MW image of the hidden "UVA" $\log \ldots \ldots$. . . . . . . . . . . . . 81

5.17 Polarization effect on imaging. . . . . . . . . . . . . . . . . . . . 83

5.18 Multi-detector image of hidden dual holes . . . . . . . . . . . . . . 85

5.19 Multi-detector image of hidden single hole . . . . . . . . . . . . . . . 86

5.20 Multi-detector sub-sampling methodology for dual blind holes . . . . . . . . 87

5.21 Multi-detector image captures of dual blind holes . . . . . . . . . . . . 88

5.22 Multi-detector resolution performance . . . . . . . . . . . . . . . . . . . . . 89

5.23 Multi-detector sub-sampling methodology for single holes . . . . . . . . . . 90

5.24 Multi-detector image captures of a blind hole . . . . . . . . . . . . . . 91

5.25 Resolution vs. stand-off distance for various imaging systems . . . . . . . . . 94

5.26 Effect of absorption on resolution vs. stand-off distance . . . . . . . . . . . 94

A.1 Measurement uncertainties of the reflect standard . . . . . . . . . . . 110

A.2 Measurement uncertainties of the thru standard . . . . . . . . . . . . 111

A.3 Measurement uncertainties of the line standard . . . . . . . . . . . . 112

A.4 Measurement uncertainties of EM properties . . . . . . . . . . . . . . . 114

B.1 Detector calibration curves . . . . . . . . . . . . . . . . 116 


\section{List of Symbols and Abbreviations}

$\begin{array}{ll}\gamma & \text { damping coefficient } \\ \delta & \text { difference } \\ \Delta & \text { image resolution } \\ \Delta_{A b b e} & \text { Abbe diffraction limit } \\ \Delta_{N F} & \text { near-field resolution limit } \\ \tan \delta & \text { dielectric loss tangent } \\ \epsilon & \text { permittivity } \\ \epsilon^{\prime} & \text { real part of complex permittivity } \\ \epsilon^{\prime \prime} & \text { imaginary part of complex permittivity } \\ \epsilon_{0} & \text { vacuum permittivity } \\ \epsilon_{e f f} & \text { effective permittivity } \\ \theta & \text { angle } \\ \theta_{T} & \text { transmitted phase } \\ \theta_{R} & \text { reflected phase } \\ \kappa & \text { extinction coefficient } \\ \mu^{\prime} & \text { wavelength } \\ \mu_{0} & \text { permeability } \\ \mu^{\prime} & \text { real part of complex permeability } \\ & \end{array}$




\begin{tabular}{|c|c|}
\hline$\mu_{e f f}$ & effective permeability \\
\hline$\xi$ & geometrical factor \\
\hline$\pi$ & mathematical constant $\pi$ \\
\hline$\rho$ & resistivity \\
\hline$\varrho$ & resolution separation distance \\
\hline$\varrho_{\text {Int }}$ & feature size of interrogating element \\
\hline$\varphi$ & phase component of complex values \\
\hline$\chi$ & variable \\
\hline$\omega$ & frequency \\
\hline$\omega_{0}$ & resonance frequency \\
\hline$\omega_{p}$ & plasma frequency \\
\hline$\omega_{p m}$ & magnetic plasma frequency \\
\hline$\omega_{\text {all }}$ & all frequencies \\
\hline$\Delta \omega$ & frequency step size \\
\hline$\varnothing$ & diameter \\
\hline$\Phi$ & phase \\
\hline $1-\mathrm{D}$ & one dimensional \\
\hline $2-\mathrm{D}$ & two dimenstional \\
\hline arb. & arbitrary \\
\hline calc. & calculated \\
\hline $\cos$ & cosine \\
\hline $\mathrm{dB}$ & decibel \\
\hline $\ln$ & natural log \\
\hline pol. & polarization \\
\hline $\sin$ & sine \\
\hline $\mathrm{Cu}$ & copper \\
\hline $\mathrm{DOF}$ & depth of focus \\
\hline
\end{tabular}


Exp. $\quad$ experiment results

EM electromagnetic

FDTD finite difference time domain

FWHM full width at half maximum

FR4 grade of a composite material commonly used for printed circuit boards

HFL high frequency lens

LFL low frequency lens

Mod. modeled results

MTM metamaterial

MW microwave

N/A not applicable

NASA National Aeronautics and Space Administration

NIM negative index of refraction material

PCB printed circuit board

RE rod element

$\mathrm{RF} \quad$ radio frequency

SMA Sub-Miniature version A, connector

SRR split ring resonator

TRL transmit, reflect, line

a unit cell spacing

c $\quad$ speed of light in a medium

$c_{0} \quad$ speed of light in vacuum

$d \quad$ split ring resonator gap

d antenna array element spacing

e Euler's number, mathematical constant

$e_{c} \quad$ electron charge

$f \quad$ focal distance 


\begin{tabular}{|c|c|}
\hline$f_{1}$ & front focal length \\
\hline$f_{2}$ & back focal length \\
\hline$f_{N F}$ & near-field standoff distance \\
\hline $\mathbf{h}$ & antenna lead height \\
\hline$i$ & imaginary $(\sqrt{-1})$ \\
\hline$j$ & induced current per unit length \\
\hline$k$ & wavevector \\
\hline$k_{0}$ & wavenumber in vacuum/free-space \\
\hline$k_{O}$ & spatial resolution of an object \\
\hline$m_{e f f}$ & effective mass of electrons \\
\hline $\mathbf{m}$ & integer \\
\hline$n$ & index of refraction \\
\hline$n_{e f f}$ & effective index of refraction \\
\hline$n_{\text {Air }}$ & index of refraction of air \\
\hline$n_{\text {Lens }}$ & index of refraction of lens \\
\hline$n^{c}$ & number of charge carriers \\
\hline$n_{e f f}^{c}$ & effective number of charge carriers \\
\hline$n^{K K}$ & solution to Kramers-Kronig method \\
\hline$q_{z}$ & propagation vector within a material \\
\hline$r$ & rod element radius \\
\hline$r^{\prime}$ & split ring resonator radius \\
\hline$r_{\text {Airy }}$ & radius of the Airy disk \\
\hline rnd & round \\
\hline$t$ & time \\
\hline$t_{C u}$ & Copper thickness \\
\hline$t_{\text {Lens }}$ & Lens thickness \\
\hline$t_{\text {sub }}$ & Substrate thickness \\
\hline
\end{tabular}




\begin{tabular}{|c|c|}
\hline$x$ & $\mathrm{x}$ position \\
\hline$y$ & y position \\
\hline$z$ & z position \\
\hline$z_{e f f}$ & effective impedance \\
\hline$B_{e f f}$ & effective total magnetic induction \\
\hline$C$ & capacitance \\
\hline $\mathrm{D}$ & lens thickness in number of unit cells \\
\hline$\vec{E}$ & electric field \\
\hline$\vec{E}_{\theta}$ & electric field polarization at angle $\theta$ \\
\hline$\vec{E}_{\theta}^{\|}$ & parallel polarized antennas at angle $\theta$ with respect to lens \\
\hline$F$ & filling fraction \\
\hline$G$ & gain \\
\hline $\mathrm{H}$ & lens height in number of unit cells \\
\hline$\vec{H}$ & magnetic field \\
\hline$H_{0}$ & applied magnetic field \\
\hline$H_{e f f}$ & effective magnetic field \\
\hline $\operatorname{Im}(x y z)$ & imaginary component of (xyz) \\
\hline $\mathbf{L}$ & antenna lead length \\
\hline $\mathcal{N}$ & number of frequency steps \\
\hline$N A$ & numerical aperture \\
\hline$S R R_{h}$ & split ring resonator trace width \\
\hline$S R R_{d}$ & split ring resonator gap size \\
\hline $\mathcal{P}$ & principal value of improper integral \\
\hline $\mathrm{R}$ & reflection coefficient \\
\hline$R_{x y z}$ & reflection coefficient of (xyz) \\
\hline$R_{e n}$ & resolution enhancement \\
\hline $\mathbb{R}$ & corrected reflection \\
\hline
\end{tabular}




$\begin{array}{ll}\mathbf{R} & \text { resistance } \\ \mathbf{R e}(x y z) & \text { real component of (xyz) } \\ \operatorname{Rod}_{h} & \text { rod element trace width } \\ \mathcal{R}_{\text {farfield }} & \text { far-field cutoff distance of an antenna } \\ S_{m n} & \text { S-parameter measurement from port } \mathrm{n} \text { to port } \mathrm{m} \\ \mathrm{T} & \text { transmission coefficient } \\ T_{x y z} & \text { transmission coefficient of (xyz) } \\ \mathbb{T} & \text { corrected transmission } \\ T_{S} & \text { Transmission for s-polarized electric field } \\ \mathrm{W} & \text { lens width in number of unit cells } \\ Z & \text { Impedance }\end{array}$




\section{Chapter 1: Introduction and}

\section{Literature Review}

\section{$1.1 \quad$ Metamaterials}

Metamaterials (MTMs) or negative index of refraction materials (NIMs) are a class of materials which are engineered to provide properties not generally found in nature. MTMs have received a lot of attention, even in mainstream media [1], due to their novel properties such as invisibility cloaking [2], negative index of refraction [3], superlensing [4] and high dispersion characteristics [5].

The ability to engineer the electromagnetic (EM) properties of a material has profound implications in the field of photonics and microwaves (MWs). Rather than deriving EM properties from chemical composition, MTMs depend on subwavelength sized resonant constituent elements that interact with incoming EM radiation. Initially, MTMs were realized at MW frequencies and were based on periodic arrays of metallic split ring resonators (SRRs) and rod elements (REs) [6].

At resonance frequency, the SRRs incite a resonance in the permeability $(\mu)$ seen by the incoming MW, forcing the real part of $\mu$ to be negative [7]. This effective negative permeability $\left(\mu_{e f f}\right)$ results from the magnetic field component $(\vec{H})$ of incident MWs inducing a current into the rings, which act as an inductive element. The current is interrupted by the gap in the SRRs, where the gap acts as a capacitive element, resulting in an equivalent LC-resonant effect [7]. LC resonance can be excited through different polarizations of the incident MW [8].

The electric field component $(\vec{E})$ of incident MWs interacts with the MTM's REs. The MTM's rods shift the Drude frequency of the metal, by effectively lowering the number of 
charge carriers as compared to bulk metal, which lowers plasma frequency. Thus net negative effective permittivity $\left(\epsilon_{e f f}\right)$ is achieved [9] for frequencies below the plasma frequency.

The constituent elements' feature sizes and substrate material determine the resonance frequency of the SRR and REs $[7,9]$. This renders MTMs scalable throughout the EM spectrum. Typically, the gap of the split ring is on the order of $1 / 10$ to $1 / 100$ of the incident radiation wavelength $(\lambda)$. The challenge is to engineer the constituent elements such that the $\epsilon_{e f f}$ and $\mu_{e f f}$ resonance regions overlap [6]. Analytical expressions relating the SRR and REs feature sizes to the $\epsilon_{\text {eff }}$ and $\mu_{\text {eff }}$ are derived in Chapter 2.

Of particular interest is the condition of $\epsilon_{e f f}=\mu_{e f f}=-1$. As the index of refraction $n$ is given by $n=\sqrt{\epsilon \mu}$ it is implied that the effective index of refraction $n_{e f f}=-1$ [10]. This provides extraordinarily unique features that are not found in nature. In 1968 Veselago [10] predicted that a flat slab of a NIM can focus diverging light from a point source down to a spot, similar to a conventional lens. The NIM slab performs this focusing through negative phase reversal of incident light and thus produces an image of the source $[4,10]$. Furthermore, this leads to the reversal of Snell's law, where the NIM material refracts light beyond the normal such that the refracted angle is the negative of the incident angle. By 2000, Pendry [4] realized that under ideal conditions, that is $n_{e f f}=-1$ in the absence of losses, the NIM lens can produce a perfect image by recovering the evanescent wave components of an object. This led to the term superlens, where a MTM lens does not adhere to fundamental limits in resolution (the diffraction limit).

Shortly after Pendry's seminal paper, the field amplifying nature of the MTM to enhance evanescent waves was experimentally verified [11-14] and imaging beyond the diffraction limit was reported $[3,6,15-18]$. This led to a great interest in metamaterial superlenses for their application towards imaging. 


\subsection{Microwave Imaging}

Microwave imaging is of interest across a broad spectrum of applications for its ability to penetrate through materials. There are many applications, such as mm-wave scanners implemented in airports [19], medical applications, MW based cancer cell detection [20,21], infrastructure monitoring $[22,23]$, and geological exploration with ground penetrating radar $[24,25]$, to name a few.

These MW techniques are primarily driven by complex digital signal processing [26]; MW imaging in particular is heavily dependent on retrieving image content from scattering effects, leading to information losses, and requiring heavy computational processing of data [27]. Traditional optical components such as lenses have been designed in the MW regime with the purpose of beam-shaping antennas to enhance antenna gain as well as use them for imaging applications [28]. Lens designs have varied from dielectric lenses [29] and Fresnel lenses [30] to transformation optics based approaches [31,32].

Imaging can be separated into the far-field and the near-field. Far-field imaging, which is typically used, is diffraction limited where as near-field imaging makes use of remnant evanescent waves and is able to resolve far below the diffraction limit. An in depth discussion on their fundamental limits of resolution is given in Chapter 2. Far-field MW imaging techniques are typically based on synthetic aperture radar measurements [20,33], which have various limitations [34], or lens elements. For near-field imaging where evanescent fields are sampled, there are several techniques besides MTMs, such as: near-field scanning microscopy [35-37], MW holography [38], near-field probes [39], and lens elements [40]. These types of systems tend to require small standoff distances less than $\lambda / 4$ [41]. Metamaterials promise to bridge the gap between diffraction limited far-field imaging and the stand-off distance requirements of near-field imaging. For a SRR and RE MTM lens based imaging system, the diffraction limit has been surpassed by showing resolutions on the order of $\lambda / 8[16,42,43]$. The MTM lenses' resolution is governed by the thickness of the lens and its internal material losses. The evanescent wave enhancing features of the MTM lens are 
highly dependent on this loss [44-46]. An interplay of acceptable losses due to MTM lens thickness and resolution gain have to be considered in designing an appropriate MTM lens. As loss mechanisms are the driving factors in determining effective resolution, it presents the opportunity to further investigate the origins of these losses and their importance to a MTM lens imaging system.

(a) Separate Source and Detector

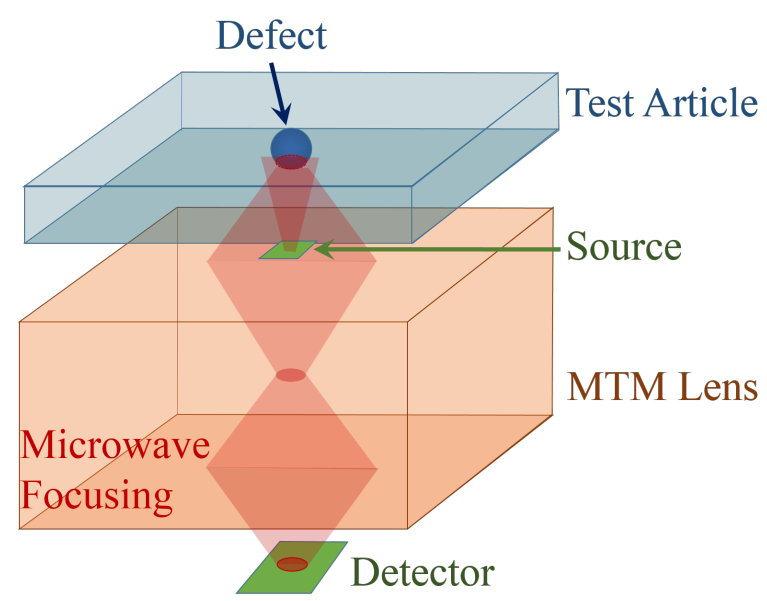

(b) Monostatic Source and Detector

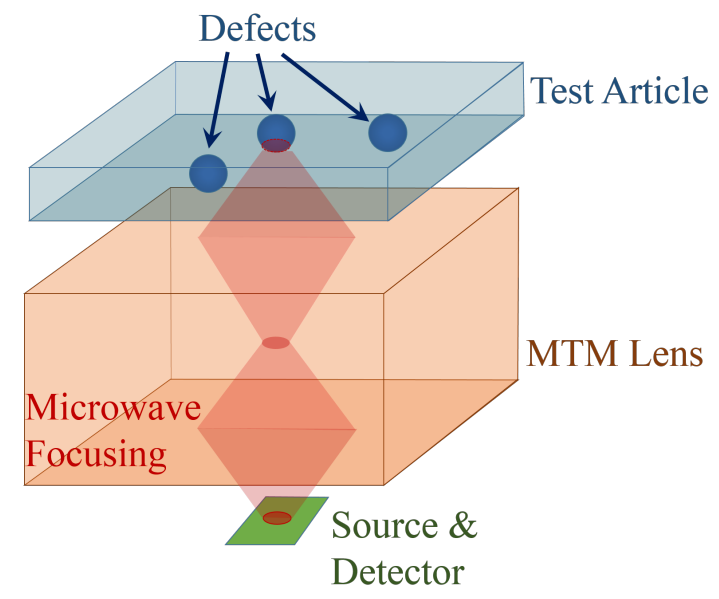

Figure 1.1: The MTM lens based MW imaging concept would allow for imaging of defects inside of a composite test article. (a) The source illuminates the test article and MWs scattered by a defect are imaged with the MTM lens onto the detector.(b) The MTM lens focuses the MWs from a source and scattering from defects would be picked up by the detector. This type of imaging device would be useful in non-destructive evaluation of structural materials.

The MTM lens based MW imaging system is of particular interest to NASA by the virtue of being a NDE technique. The non-ionizing radiation of microwaves would be able to penetrate into materials under test and the subwavelength focusing capabilities of the MTM lens would allow for imaging of small defects. Of particular interest would be the evaluation of composites as they are commonly used in aircraft, heat-shields, and UAVs. Under normal working loads delaminations and defects in the composite could lead to catastrophic failure, thus compromising the structural integrity of the vehicle. A MW MTM lens based MW NDE system would directly benefit the aviation safety community and others which lack practical inspection techniques for subsurface defect analysis. 


\section{$1.3 \quad$ Modeling}

Modeling of MW MTMs is primarily done with finite difference time domain (FDTD) or finite element analysis methods. These were used to predict the resonant characteristics of MTM constituent elements and thus determine the EM properties of the bulk MTM [8,47-49]. Furthermore, modeling was used to verify the MTM's imaging characteristics and was critical in the investigation of the limitations of MTMs for sub-diffraction limited imaging $[44,50]$. Modeling also is instrumental into giving insight into MTMs interaction with EM waves from which a variety of applications with novel MTM architectures have been predicted. Literature has reported on modeling MTM uses in radomes [51], zero reflection surfaces [52], high-reflection coatings [53], and reflection mode imaging [54,55].

\subsection{Problem Statement}

MW MTMs possess unique properties that can address the demand for novel non-destructive evaluation techniques. MWs are non-ionizing, allow for greater material penetration [56] and ease of use $[57,58]$, as compared to optical, infrared or ultrasound techniques. Commonly used MW techniques are either resolution limited due to diffraction effects (far-field sensing) or impractical due to very small standoff distance requirements (near-field sensing). The evanescent field amplifying nature of MTMs allows for overcoming the diffraction limit [4], thus permitting subwavelength resolution sensing of defects at convenient macro-scale working distances. While fundamental electromagnetic properties of MTMs have been investigated with respect to sub-diffraction limited imaging, this dissertation investigates the use of practical MTM lenses towards demonstrating a novel MTMs based imaging system with subwavelength resolution.

Previous has work focused on establishing the resolution limits of MTMs [50], by using thin lenses [16], point sources as objects for imaging, and operating in transmission mode. By

making use of and surpassing previously established single point detection capabilities [42], 
this dissertation aims to evaluate MTM capabilities in the context of a subwavelength resolution imaging system. This work focuses on making use of transmission and reflection mode imaging, and carries out modeling and experiments to evaluate the capabilities and feasibilities of the MTM based system.

\subsection{Objectives and Research Goals}

As MTM lenses are presented in literature to overcome the diffraction limit, without stringent near-field stand off distance requirements, a MTM based MW imaging system presents a unique solution to high resolution imaging capabilities. This dissertation studies the MW imaging characteristics of a MTM lens based imaging system. In order to perform an accurate feasibility demonstration, a real world MTM lens has been designed, fabricated, and evaluated. This research builds upon a previously established MW MTMs based singlepoint [42] detection system and surpasses its abilities through point-to-point as well as multi-point array 2-D imaging with subwavelength resolution. Various electromagnetic properties of MTM lenses have been evaluated and the subwavelength resolution capabilities have been demonstrated. The imaging performance is further enhanced through measurement and utilization of polarization properties of the metamaterial lenses. The 2-D imaging system's effectiveness is assessed using metal and composite targets. 


\section{Chapter 2: Theoretical Background}

In positive index of refraction materials, inclusions of chemical elements, surface morphology, or ordered states of alternating materials are used to create an effective media which exhibits a change in index of refraction as compared to bulk materials. MTMs, however, use precisely engineered resonant structures made of conductors that are sized well below the wavelength. These resonant elements determine the electromagnetic response of the material and drive the index of refraction. Negative indices of refraction are made possible by designing these constituent elements such that incoming microwaves experience a material that exhibits negative effective permeability and negative effective permittivity. Index of refraction is given from $n=\sqrt{\epsilon \mu}$. Negative index of refraction is defined such that the components permeability and permittivity are both negative.

In nature it is possible to have materials with either $\epsilon$ or $\mu$ being negative. Many metals exhibit $-\epsilon$, whereas $-\mu$ occurs rarely in some magnetic materials at low frequencies [59]. However, a natural material with combined negative $\mu$ and negative $\epsilon$ has not been found. In the case that either $\mu$ or $\epsilon$ is negative, incident electromagnetic radiation does not propagate into the material, as the index of refraction becomes imaginary. This presents a forbidden band and all incident propagating radiation reflects at the interface and non-propagating radiation becomes evanescent. In metamaterials where both components are negative, propagating modes are allowed because the index of refraction becomes real. The sign convention for negative index of refraction comes from $\epsilon=\mu=-1$, where both can be rewritten as $\epsilon=\mu=e^{i \pi}$. From this the index of refraction follows as $n=\sqrt{\epsilon \mu}=e^{i \pi / 2} e^{i \pi / 2}=-1$, hence the sign convention establishes that negative $\mu$ and negative $\epsilon$ lead to negative index of refraction. 


\subsection{Metamaterial Constituent Elements}

In microwave metamaterials there are two different resonant structures: rod type structures which contribute to an effective negative permittivity and SRR structures which provide for net negative permeability. The challenge lies in engineering an overlapping resonant response between these independent elements such that the net index of refraction is negative.

\subsubsection{Rod elements and $-\epsilon_{e f f}$}

Pendry et al. realized that by making use of metals' interaction with incident radiation, negative permittivity could be realized at microwave frequencies [9]. The challenge arose to lower the plasma frequency $\omega_{p}$ of the metal to $\mathrm{GHz}$ frequencies. In order to do so Pendry used an array of subwavelength spaced metallic rods [9]. The aggregate of this rod array under effective medium theory changes the behavior of a bulk metal such that the plasma frequency is suppressed to $\mathrm{GHz}$ frequencies. The following derivation shows the $\epsilon(\omega)$ relationship to the feature sizes of the rod array.

$$
\epsilon(\omega)=1-\frac{\omega_{p}^{2}}{\omega(\omega+i \gamma)}
$$

This is the Drude model for a bulk metal, where the permittivity $\epsilon$ changes as a function of frequency $\omega$ under a resonance effect governed by the plasma frequency $\omega_{p}$ and the damping coefficient $\gamma[60]$. The Drude model comes from the interaction of an incident electromagnetic wave with the free carriers in a metal. The incident wave polarizes the free electrons and induces a dipole moment. By summation of all dipole moments and their restorative forces over the number of charge carriers $n^{c}$, the relation 2.1 is found. In the Drude model, the plasma frequency $\omega_{p}$ is directly related to the number of effective charge carriers $n_{\text {eff }}^{c}$, the electron charge $e_{c}$, permittivity of vacuum $\epsilon_{0}$ and the effective mass of the electrons $m_{e f f}$.

$$
\omega_{p}^{2}=\frac{n_{e f f}^{c} e_{c}^{2}}{\epsilon_{0} m_{e f f}}
$$


By using a medium of vertically aligned rods (see fig. 2.1), in the limit of the rod radius $r$ and spacing $a$ being much smaller than the wavelength $\lambda(a \& r \ll \lambda)$ and that the radius is much smaller than the rod spacing $(r \ll a)$, the effective number of charge carriers $n_{\text {eff }}^{c}$ is geometrically modified. A change in $m_{e f f}$ is also noted. This change is derived from the change in momentum of electrons flowing on the surface of the rods in the medium, a more detailed derivation of $m_{e f f}$ is given in [59]:

$$
n_{\text {eff }}^{c}=n^{c} \frac{\pi r^{2}}{a^{2}} \quad \text { and } \quad m_{e f f}=\frac{\mu_{0} r^{2} n_{e f f}^{c} e^{2}}{2} \ln \left(\frac{a}{r}\right)
$$

The $m_{\text {eff }}$ is dependent on the permeability of vacuum $\mu_{0}$, radius of the rod $r$, number of effective charge carriers $n_{\text {eff }}^{c}$, the number of charge carriers $n^{c}$, the electron charge $e_{c}$ and the rod spacing $a$. By substituting 2.3 into 2.2, 2.4 is obtained.

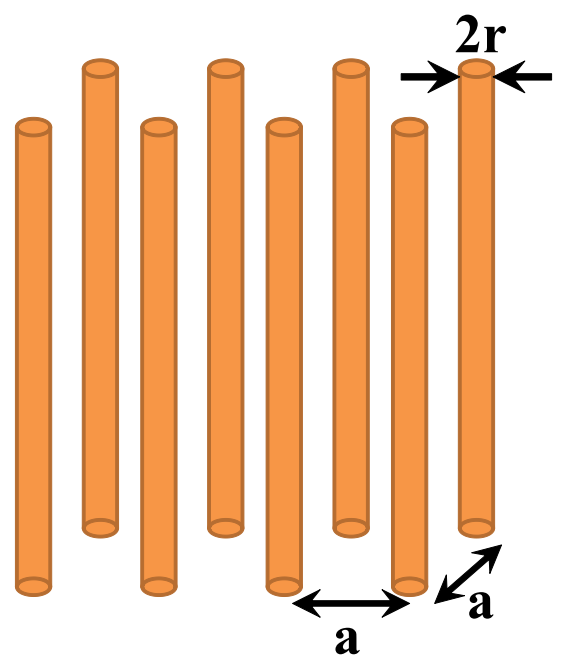

Figure 2.1: Drawing of the basic RE architecture, with unit cell size (a), and RE radius (r). Incident $\vec{E}$ is parallel to the rod array. An array of metal RE suppresses the $\omega_{p}$ based on the array's geometrical factors a and r.

$$
\omega_{p}=\left(\frac{2 \pi c_{0}^{2}}{a^{2} \ln \left(\frac{a}{r}\right)}\right)^{\frac{1}{2}}
$$

Equation 2.4 shows that a rod medium can be used to suppress the plasma frequency of a metal which now depends on the rod radius $r$, separation $a$ of the rods, and the speed of 
light in vacuum $c_{0}$. By making use of the finite conductivity of rods, the damping coefficient $\gamma$ is replaced by

$$
\gamma=\epsilon_{0} \rho \frac{\omega_{p}^{2} a^{2}}{\pi r^{2}}
$$

[59], where $\rho$ is the resistivity of the bulk metal. By substituting $\omega_{p}$ and $\gamma$, the $\epsilon_{e f f}$ can be written as

$$
\epsilon_{e f f}=1-\frac{\omega_{p}^{2}}{\omega\left(\omega+i\left(\epsilon_{0} \rho \frac{\omega_{p}^{2} a^{2}}{\pi r^{2}}\right)\right)}
$$

This shows that an effective permittivity $\epsilon_{\text {eff }}$ can be tailored throughout the electromagnetic spectrum by making use of the geometrical arrangement of rods that are very small with

respect to the operating wavelength. For frequencies below $\omega_{p}$ the effective permittivity is negative. At values above $\omega_{p}, \epsilon_{e f f}$ becomes positive. By precisely controlling the rod array $\epsilon_{e f f}=-1$ can be achieved at microwave frequencies.

\subsubsection{Split Ring Resonators and $-\mu_{e f f}$}

Negative permeability only exists under very specific circumstances [59], thus proving to be much more difficult to attain than negative permittivity. Pendry et al. conceived a resonant structure based on an array of subwavelength sized concentric split rings to achieve negative permeability [7]. These structures act equivalently to an inductor-capacitor resonant circuit. They consist of an inductive element, the rings, and a capacitive element, the gap. Exciting the SRRs at the resonance frequency gives an LC-resonance which drives the effective permeability to be negative. The following equations derive the resonant effective permeability of an array of SRRs. The SRRs are excited with an incident electromagnetic field, polarized such that the magnetic field component of the wave is through the axis of the SRR elements. The $\mu_{\text {eff }}$ is given by treating the SRRs as a stack of concentric metallic cylinders with a split in a unit cell array. The ring internal radius is denoted as $r^{\prime}$, the ring separation and ring gap as $d$, and the unit cell size as $a$. By finding the effective total magnetic induction $B_{\text {eff }}$ 
and effective magnetic field strength $H_{\text {eff }}, \mu_{\text {eff }}$ is given by 2.6.

$$
\mu_{e f f}=\frac{B_{e f f}}{\mu_{0} H_{e f f}}
$$

Outside the stack of cylinders, $B_{\text {eff }}$ and $H_{\text {eff }}$ are found respectively by averaging the magnetic induction $(B)$ over the area of the unit cell and averaging the magnetic field $(\vec{H})$ along the line edge of the unit cell [59]. This results in 2.7, where $B_{\text {eff }}$ depends on the permeability of vacuum $\mu_{0}$ and the applied magnetic field $H_{0} . H_{\text {eff }}$ is given by $H_{0}$ and the geometrical contribution of the concentric rings multiplied by $j$, and the induced current per unit length.

$$
B_{\text {eff }}=\mu_{0} H_{0} \quad \text { and } \quad H_{e f f}=H_{0}-j \frac{\pi r^{\prime 2}}{a^{2}}
$$

In order to find the applied magnetic field $H_{0}$ as it relates to the SRR geometry and incident wave, the electromotive force around the SRRs is calculated [59]. The term on the left of 2.8 relates to the axial magnetic field inside the cylinders and the term on the right relates to the current on the rings and across the gaps with capacitance $C$. Equation 2.8 assumes that $d \ll r^{\prime}$ and is solved for $H_{0}$ in 2.9 .

$$
\begin{gathered}
-i \mu_{0} \pi r^{\prime 2} \omega\left(H_{0}+j\left(1-\frac{\pi r^{\prime 2}}{a^{2}}\right)\right)=j\left(2 \pi r^{\prime} \rho-\frac{1}{i \omega C}\right) \\
H_{0}=j\left(-1+\frac{\pi r^{\prime 2}}{a^{2}}+\frac{i 2 \rho}{\mu_{0} \omega r^{\prime}}-\frac{1}{\mu_{0} \pi r^{\prime 3} \omega^{2} C}\right) \quad \text {, where } \quad C=\frac{\epsilon \pi r}{3 d} \epsilon_{0}
\end{gathered}
$$

The incident magnetic field is found to be dependent on the current density $j$, the geometrical factors $r^{\prime}, a$, the resistivity of the metal $\rho$, the permeability of vacuum $\mu_{0}$, the frequency of the incident EM wave $\omega$ and the capacitance of the SRR gaps $C$. $C$ is also dependent on the permittivity of the dielectric inside the gaps $\epsilon$. By substituting 2.7 and 2.9 into 2.6 the $\mu_{\text {eff }}$ of the SRRs is derived.

$$
\mu_{e f f}=1-\frac{\pi r^{\prime 2} / a^{2}}{1-\frac{3 d c_{0}^{2}}{\epsilon \pi^{2} r^{\prime 3} \omega^{2}}+\frac{i 2 \rho}{\mu_{0} \omega r^{\prime}}}
$$


Analysis of $\mu_{\text {eff }}$ shows a resonant characteristic with a resonance frequency of $\omega_{0}$ and a

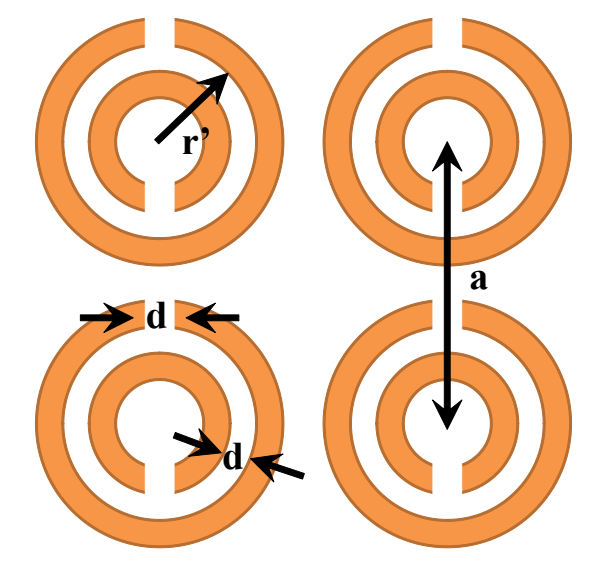

Figure 2.2: Drawing of the basic SRR architecture, with unit cell size (a), SRR gap (d), and ring radius ( $\left.\mathrm{r}^{\prime}\right)$. The geometrical arrangement of SRRs induces $\mu_{\text {eff }}$ based on the geometrical factors in the SRRs feature sizes.

magnetic plasma frequency $\omega_{m p}$.

$$
\omega_{0}=\left(\frac{3 d c_{0}^{2}}{\epsilon \pi^{2} r^{\prime 3}}\right)^{\frac{1}{2}} \quad \text { and } \quad \omega_{m p}=\left(\frac{3 d c_{0}^{2}}{\left(1-\frac{\pi r^{\prime 2}}{a^{2}}\right) \epsilon \pi^{2} r^{\prime 3}}\right)^{\frac{1}{2}}
$$

Evaluating $\mu_{e f f}$ for its resonance frequency reveals that $\mu_{e f f}$ diverges at $\omega_{0}$ and transitions to zero at $\omega_{m p}$. Frequencies well below $\omega_{0}$ and well above $\omega_{m p}$ return the $\mu_{\text {eff }}$ to unity. For frequencies between $\omega_{0}$ and $\omega_{m p}$, the real part of $\mu_{e f f}$ is negative. The bandwidth for this net negative permeability is given by the filling fraction $\left(F=\frac{\pi r^{\prime 2}}{a^{2}}\right)$ of the SRR geometry. The peak absolute value of the $\mu_{\text {eff }}$ occurs at $\omega_{0}$ and is constrained by the material losses of the SRRs. Through precise control over the SRR geometry and substrate material, $\mu_{e f f}=-1$ can be achieved at a desired frequency, throughout the electromagnetic spectrum.

\subsubsection{Polarization Excitation}

Incident polarization plays a major role in the resonance effects of metamaterial constituent elements. For the rod elements, a depression of the Drude frequency is only possible with the electric field of the incident EM wave polarized along the axis of the rods. For other

polarizations, only the $\vec{E}$-field vector component that is parallel to the rods contributes to the 
resonance suppression. For the SRRs the polarization effects are more complex. True negative permeability is achieved with the incident wave polarized in such a way that the magnetic field points through the rings and the electric field is perpendicular to the SRR gaps [8,61]. Alternate polarization, where the incident polarization of the electric field is along the gaps and the wave propagation along the axis of the SRRs, can also excite a response [62]. In this configuration the excitation results in a predominantly electric response, which through magneto-electric coupling interacts with the incident MW radiation and provides a $-\mu_{\text {eff }}[61]$. In building negative index media, the polarization of the incident wave has to be such that the negative permittivity from the rod components and negative permeability contribution of the SRRs are excited simultaneously.

\subsection{Metamaterials Imaging}

The imaging properties of MTM lenses are governed by the interaction between EM waves and the negative index of refraction of the medium. This gives rise to a variety of interesting phenomena. The principle of particular importance to this dissertation is the metamaterial flat lens, also known as the superlens. This lens focuses both propagating and evanescent waves in the image plane and thus creates imaging capabilities below the diffraction limit.

\subsubsection{Focusing}

The MTM flat lens' ability to focus diverging light is described by Snell's law. Snell's law comes from setting up the boundary conditions for an incoming EM wave interacting with a medium. Part of the incident wave is transmitted and some of the wave is reflected at the interface. Crucially, Snell's law provides the angle of refraction at the interface of any two materials, where $n_{1} \sin \left(\theta_{1}\right)=n_{2} \sin \left(\theta_{2}\right), n_{1}$ being the index of the first material, $\theta_{1}$ the angle of incidence with respect to the normal, $n_{2}$ the index of the second material, which the EM wave travels into, and angle of refraction $\theta_{2}$. For ordinary materials, the refracted angle can 
become very close to the normal of the interface, however may never cross it. Negative index of refraction materials show a refraction angle that is negative, that is, the transmitted wave is on the other side of the normal. This presents an interesting case for designing optical components such as lenses. For one, the geometrical shapes are switched: a convex lens with negative index of refraction acts like a concave lens of positive index and a concave NIM lens acts as a convex lens. More interesting is the possibility of a flat slab of negative index material at $n=-1$ acting as a lens. Without curvature, this paves the way for very compact lens elements, which could drastically simplify designs for imaging. The flat lens shown in figure 2.3 has its merits and drawbacks. The relationship between the focal lengths $f_{1}$ and $f_{2}$ and the lens thickness $t_{\text {Lens }}$ suggests that the lens does not have a fixed focal spot position and an image is only formed when the object is placed near the lens such that $t_{\text {Lens }}=f_{1}+f_{2}$ is preserved. Moreover, in a practical application, the tuning of the $\epsilon_{\text {eff }}$ and $\mu_{\text {eff }}$ might not result in an exact $n_{\text {eff }}=-1$. Therefore, for practical applications, Wilson et al. have derived the relationship for imaging requirements as shown in eq. 2.12 for arbitrary negative index of

(a) $n=-1$ Lens

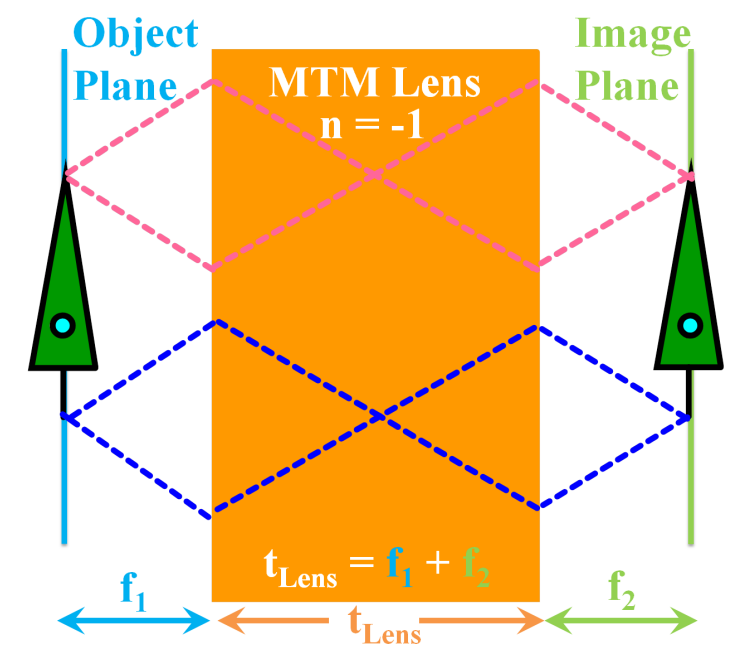

(b) Ordinary Thin Lens ( $\mathrm{n}$ is positive)

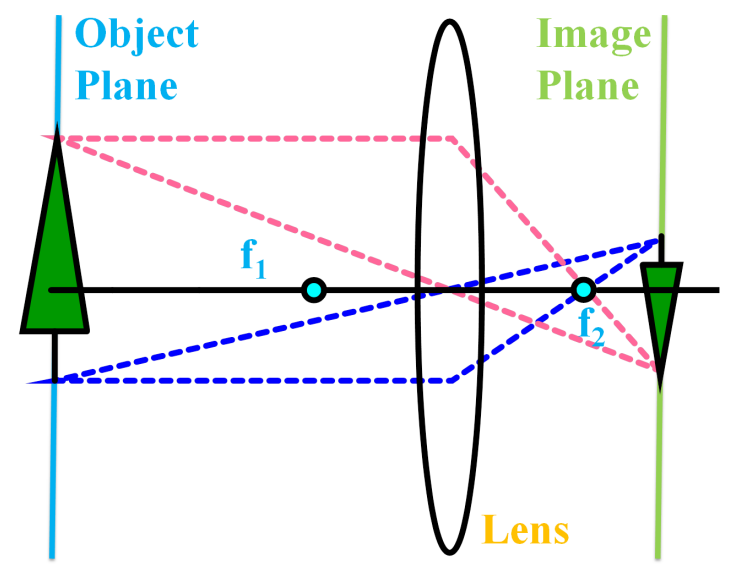

Figure 2.3: (a) Ray transmission through a negative index lens. The condition for lensing is that the object to lens distance $f_{1}$ is less than the lens thickness $\left(t_{\text {lens }}\right)$. The lens to image plane distance is calculated from $t_{\text {lens }}=f_{1}+f_{2}$. (b) shows the ray-transmission through an ordinary thin lens. Unlike normal lenses, the MTM lens does not invert the image. 
refraction [63]:

$$
f_{2}=\frac{n_{\text {Air }} \cos (\theta) t_{\text {Lens }}}{\sqrt{n_{\text {Lens }}^{2}-n_{\text {Air }}^{2} \sin ^{2}(\theta)}}-f_{1}
$$

The equation relates the focal length of the image plane $f_{2}$ to the index of refraction of the surrounding medium, $n_{\text {Air }}$, index of the lens $n_{\text {Lens }}$, the angle the incident source (object) makes with the lens $\theta$, the lens thickness $t_{\text {Lens }}$, and the object standoff distance to the lens, also known as the front focal length $f_{1}$. However, focusing of propagating waves alone does not aid in overcoming the diffraction limit. For that, the evanescent waves need to be taken into account.

\subsubsection{Evanescent Wave Enhancement}

Metamaterials have received a lot of research interest for their ability to enhance the evanescent waves of an object such that an ideal image can be reconstructed. Evanescent waves are the non-propagating wave components that occur when an EM wave interacts with an object. These waves carry the subwavelength sized fine structure information of the object; however, they decay exponentially in amplitude from the object interface, whereas propagating waves decay in phase [4]. Due to this characteristic, the information is lost for ordinary imaging applications, which ultimately contributes to the limits in resolution. Fortunately, MTMs have been proven to recover the fine structure information and reproduce it in the image plane [4]. This does not violate energy conservation, as evanescent waves do not transport energy [4]. The special case of $\epsilon=-1$ and $\mu=-1$, denoting a lossless material of $n=-1$, is also called a superlens $[4,45]$.

The following derivation leads through the recovery of the evanescent waves of a 2-D line source with S-polarization due to a $n=-1$ lens. Similar arguments for a P-polarized wave can be made and are derived in [4]. The 2-D line source is setup in free space in front of a planar slab of $n=-1$ material, where $x$ denotes the direction parallel to the NIM and $z$ is 
the direction normal to the NIM. The electric field has the following expression:

$$
E(x, z, t)=\sum_{k_{x}} E\left(k_{x}\right) e^{i k_{z} z+i k_{x} x-i \omega t}
$$

where $E\left(k_{x}\right)$ is the Fourier transform of the spatial variation of the source, $k_{x}$ and $k_{z}$ are the wavevector components, $\omega$ the frequency of the wave and $t$ is time. The homogeneous wave equation outside of the slab gives the following dispersion relation where the wave vector components are related to the frequency.

$$
k_{z}=\sqrt{\frac{\omega^{2}}{c^{2}}-k_{x}^{2}}
$$

This general equation holds for both propagating and non-propagating components of the plane wave. For $k_{x}<\frac{\omega}{c}$, or wavelengths smaller than $1 / k_{x}$, the information contained in $E\left(k_{x}\right)$ is propagating. Conversely, for $k_{x}>\frac{\omega}{c}$, information contained in $E\left(k_{x}\right)$ is evanescent. This means that feature sizes below the interrogating wavelength are lost. Another consequence of the special case where $\epsilon=\mu=-1$ is its perfect impedance $(Z)$ match to free space. Without reflection components at the interface with the lens and no internal losses, propagating waves are transmitted through the negative index medium unhindered.

$$
Z=\sqrt{\frac{\mu \mu_{0}}{\epsilon \epsilon_{0}}}
$$

In the case of the S-polarized $\vec{E}$-field the transmission $T_{S}$ is related to the thickness of the lens $t_{\text {Lens }}$ and values of $\epsilon$ and $\mu$, where $q_{z}$ is the propagation vector within the slab. The phase factor $e^{-k_{z} t_{L e n s}}$ has been absorbed into $T_{S}$.

$$
T_{S}^{-1}=\cos \left(q_{z} t_{\text {Lens }}\right)-\frac{i \sin \left(q_{z} t_{\text {Lens }}\right)}{2}\left(\frac{\mu k_{z}}{q_{z}}+\frac{q_{z}}{\mu k_{z}}\right) \quad \text { where } \quad q_{z}=\sqrt{\epsilon \mu \frac{\omega^{2}}{c^{2}}-k_{x}^{2}}
$$


For evanescent wave components, the dispersion relation dictates that $k_{x}>\sqrt{\epsilon \mu} \omega / c>\omega / c$ is chosen. The resulting transmission coefficient takes the following form:

$$
T_{S}=\left(e^{q_{z} t_{\text {Lens }}}\left[\frac{1}{2}+\frac{1}{4}\left(\frac{\mu k_{z}}{q_{z}}+\frac{q_{z}}{\mu k_{z}}\right)\right]+e^{-q_{z} t_{\text {Lens }}}\left[\frac{1}{2}-\frac{1}{4}\left(\frac{\mu k_{z}}{q_{z}}+\frac{q_{z}}{\mu k_{z}}\right)\right]\right)^{-1}
$$

In ordinary materials with positive $\mu$ the first term dominates the transmission and the fine structure information decreases exponentially. In the special case of a superlens $\left(k_{z}=q_{z}\right.$, and $\epsilon=\mu=-1$ ), the transmission coefficient becomes $T_{S}=e^{+q_{z} t_{\text {Lens }}}$ and all of the non-propagating field components grow exponentially throughout the thickness of the lens. Therefore, in combination with the superlens focusing the propagating waves, all of the source fields are reproduced exactly in the image plane $[4,45]$. The experimental proof of concept has been reported in literature [11-16].

\subsection{Fundamental Limits of Imaging}

Imaging is separated into two regions, the far-field and the near-field. Far-field imaging is limited in resolution, due to loss of evanescent waves, whereas the near-field retains remnant evanescent waves and is limited by standoff distance. Metamaterials based imaging could bridge the difference between the near-field and the far-field by relaxing stringent standoff distance requirements and retaining resolution below the diffraction limit.

\subsubsection{Far-Field Imaging}

In the far-field, the Fraunhofer diffraction regime, the fundamental limit of resolution is determined by the diffraction limit $[60,64,65]$. This limit specifies the minimum separation distance between two objects that any conventional far-field imaging system can resolve. In the theoretical limit of an aberration-free, and circularly symmetric lens imaging a point source, the result is a diffracted image with a finite spread. The spreading is due to the 
Fraunhofer diffraction pattern of the exit pupil of the lens [65]. The spread function is given by the Airy function, which consists of a first order Bessel function, exhibiting the widely recognized Airy pattern [60]. Of particular interest in the Airy diffraction pattern is the central bright spot known as the Airy disk, with a radius $r_{\text {Airy }}$ given by the following equation.

$$
r_{\text {Airy }}=0.61 \frac{\lambda}{N A}
$$

$N A$ is the numerical aperture of the lens, $N A=n \sin \theta$, given by the index of refraction of the lens $n$ and the collection angle of the lens $\theta$. When two point sources of equal intensity are imaged simultaneously, they are considered to be resolved when their diffraction patterns are distinguishable from each other. The limit for resolution, the minimum separation distance between the patterns, is given by the Rayleigh criterion. The Rayleigh criterion defines the resolution limit at the separation distance of $\varrho=r_{\text {Airy }}$, where the peak intensity of the first source's diffraction pattern coincides with the first null of the second source's pattern. An example of dual holes imaged with the Rayleigh criterion is provided in fig. 2.4a. Beyond the Rayleigh criterion is the Sparrow Limit (see fig. 2.4c), with $\varrho=0.78 r_{\text {Airy }}$. Any separation less than the Sparrow limit gives a sum of two diffraction patterns that are indistinguishable from each other [65]. The most widely adopted fundamental limit of resolution is Abbe's diffraction limit (see fig. 2.4b) which is given below [64].

$$
\Delta_{\text {Abbe }}=0.5 \frac{\lambda}{n \sin \theta}=0.82 r_{\text {Airy }}
$$

In the case of a $n_{e f f}=-1$ superlens, the far-field resolution limit reduces to $\Delta_{A b b e}=\frac{\lambda}{2}$. The challenge for the metamaterial lens based imaging system is to overcome this resolution limit.

\subsubsection{Near-Field Imaging}

Abbe's diffraction limit can be overcome by imaging an object in the near-field. The near-field region is governed by Fresnel diffraction, and generally requires image standoff distances less 
(a) Rayleigh Criterion

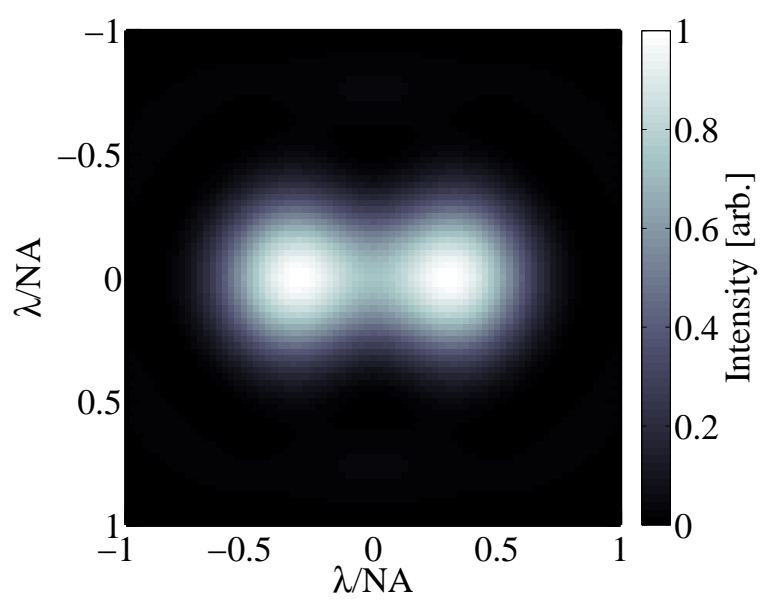

(c) Sparrow Limit

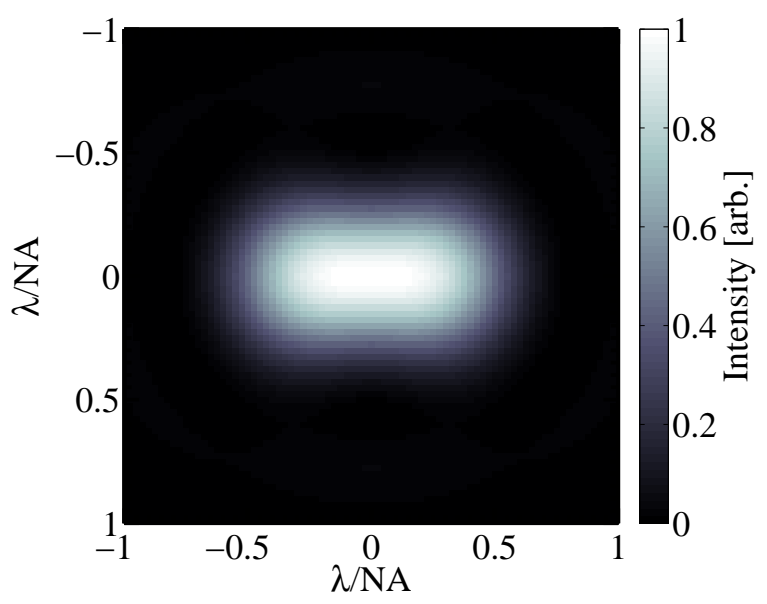

(b) Abbe Limit

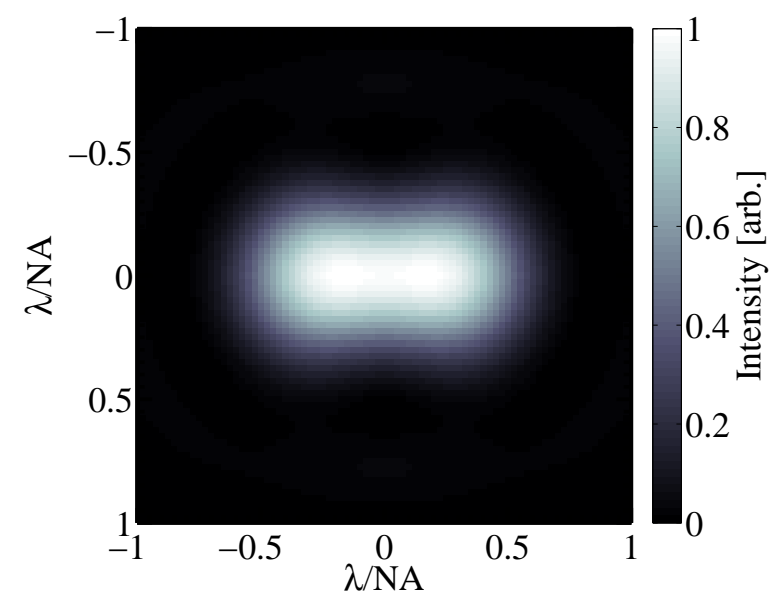

(d) Comparison

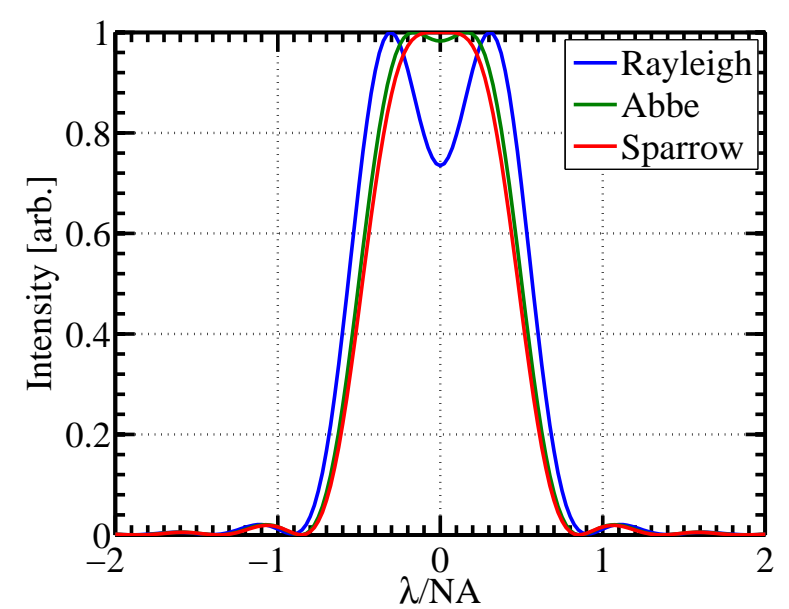

Figure 2.4: Fraunhofer diffraction patterns from two circular apertures imaged by a lens with $\mathrm{NA}=1$. This highlights the resolution criteria for distinguishing two object. (a) image of dual holes separated by $r_{\text {airy }}$ (Rayleigh criterion). (b) image of dual holes at the Abbe diffraction limit. (c) image of dual holes at the Sparrow limit. (d) relative comparison of the three resolution limits.

than $\lambda / 4$ [41]. Near-field imaging is heavily dependent on the near-field interaction between the source and object. Deeply subwavelength resolution $(\lambda / 20)$ images can be obtained by creating evanescent waves with very high spatial frequencies and recovering their interaction with the object [37]. In a near-field imaging system, the spatial resolution of evanescent waves from the source directly determine the resolution limit. As the fine structure information 
contained in the evanescent waves can be no smaller than the minimum feature size of the interrogating element $\left(\varrho_{\text {Int }}\right)$, the resolution is on the order of $\varrho_{\text {Int }}[36,37]$. In order to resolve the fine structure of the object, $\varrho_{\text {Int }}$ not only has to be much smaller than the wavelength $\lambda$ but also has to be much smaller than the spatial resolution of the object $k_{O}$ such that $k_{O} \varrho_{\text {Int }}<<1$. Furthermore, evanescent waves decay exponentially on the length scale of the size of the scatterer $[36,37]$. Therefore, the maximum obtainable resolution $\Delta_{N F}$ and maximum standoff distance $f_{N F}$ is given by the following.

$$
\Delta_{N F}=\varrho_{\text {Int }} \quad \text { and } \quad f_{N F}=\varrho_{\text {Int }}
$$

Due to these stringent requirements, near-field imaging below the diffraction limit is severely limited by the interrogating element size. In order to beat the diffraction limit, the size of the source element has to be smaller than $\lambda / 2$, while requiring a standoff distance of less than $\lambda / 2$. Unlike conventional imaging, near-field imaging is achieved by point to point interrogation, which makes it very slow in comparison to far-field imaging.

\subsubsection{Metamaterial Lens}

In practice it is very difficult to achieve an ideal lossless metamaterial lens. This results in the overall defocusing of the image. Losses inside the lens come from the material parameters of the constituent elements, their resonance characteristics, and also from mismatches in alignment of the resonance frequency between the SRR and RE elements. Furthermore, literature shows that the resolution of the image is also highly dependent on the geometry of the MTM lens $[44,45]$. The resolution enhancement $R_{e n}$ due to amplified evanescent waves for a MTM lens based on an ordered array of resonant elements is given as follows [45].

$$
R_{e n} \equiv \frac{\lambda}{\lambda_{\min }}=-\frac{\ln |\delta \mu|}{2 \pi} \frac{\lambda}{d}
$$


The resolution enhancement, which is the ratio of interrogating wavelength $\lambda$ to minimum feature size $\lambda_{\text {min }}$, is dependent on the change in permeability $\delta \mu$ from the ideal $\mu=-1$, and the lens thickness $d$. The dominant term is the ratio $\lambda / d$. However, this formula only holds for $d \ll \lambda$. In order to surpass the diffraction limit, the thickness of the lens, (which in turn dictates the maximal standoff distance) has to be very small with respect to $\lambda$ and material losses have to be minimized [66]. In a practical application, however, the focal distance of the MTM lens would have to be larger than the wavelength in order to bridge the difference between near-field and far-field imaging. For this case a much more generalized equation is presented [46]. The focal distance $f$, i.e. the thickness of the MTM lens $\left(t_{\text {lens }}=2 f\right.$ for a symmetrical focusing arrangement), is related to the image resolution $\Delta$ in the presence of losses stemming from imaginary permittivity $\left(\epsilon^{\prime \prime}\right)$ and imaginary permeability ( $\left.\mu^{\prime \prime}\right)$, a geometrical factor $\xi \approx 0.6[46,67]$ due to the image source, and the wavelength.

$$
f_{\{\vec{E}\}}=\frac{\Delta \ln \left[\frac{4\left(\xi^{2} \lambda^{2} / \Delta^{2}-1\right)}{\left\{\epsilon^{\prime \prime}\right\}+\left(2 \xi^{2} \lambda^{2} / \Delta^{2}-1\right)\left\{\mu^{\prime \prime}\right\}}\right]}{4 \pi \sqrt{\xi^{2}-\Delta^{2} / \lambda^{2}}}
$$

The relationship specifically calculates the resolution as the full width at half maximum of the image and takes into account source polarization $\vec{E}$. Equation 2.22 does however assume real part of $\epsilon=-1$ and $\mu=-1$. In spite of this, equation 2.22 is a much more robust model for the assessment of the near-field focusing capability of a planar MTM lens with real losses. While losses can be minimized through careful consideration of material properties and other techniques (such as optical parameter amplification [68] or geometrical arrangement [67]), it is difficult to attain resolution beyond the diffraction limit with realistic MTM lenses. However, compared to near-field imaging, with its complex image retrieval and limited standoff distances, and far-field imaging with very large lens apertures, the metamaterial lens provides a practical solution for a simplified near-field imaging without the use of bulky far-field optical elements. 


\section{Chapter 3: Metamaterial Lens}

\section{Modeling, Design, Fabrication, and}

\section{Characterization}

The MTM lenses described in this dissertation have been designed and fabricated previously [69], and are based on SRR and RE elements. This type of MTM is widely used at MW frequencies and draws its design back to the original MTM [6]. The lenses have been designed by use of analytical formulas as well as Ansoft HFSS (C) modeling software to overlap the $\mu_{e f f}$ and $\epsilon_{e f f}$ resonant regions such that negative $n_{\text {eff }}$ is achieved.

This research investigates two lenses of different design frequencies, a low frequency lens (LFL) exhibiting resonance at $3.63 \mathrm{GHz}$ and a high frequency lens (HFL) with resonance at $16.65 \mathrm{GHz}$.

\subsection{Modeling}

The propagation mechanisms for MWs inside of the metamaterial lenses are complex $[8,61,70]$ and it is best to use computational modeling to further refine the MTM lens design, understand its imaging characteristics, and analyze its electromagnetic properties. Full wave computational analysis was performed with commercially available RF and microwave modeling package CST Microwave Studio (c). Simulations are set-up to run with CSTs Time-Domain solver. Due to the complex nature of the MTM unit cells, different modeling methodologies were employed for evaluating its electromagnetic properties and lens performance. In addition to analyzing the MTM lenses, the same software was used to design and evaluate antenna parameters for the multi-element detector. 


\subsubsection{Modeling of Electromagnetic Properties}

For transmission and reflection properties, an open unit cell model with perfect unit cell repetition was excited with a plane wave. Electric field probes set in front of and behind the structures recorded the reflected and transmitted electric fields. The simulation replicated the height and width of the MTM structure to infinity, while the thickness of the fabricated lens was replicated by modeling 10 unit cells along the propagation direction. The model was

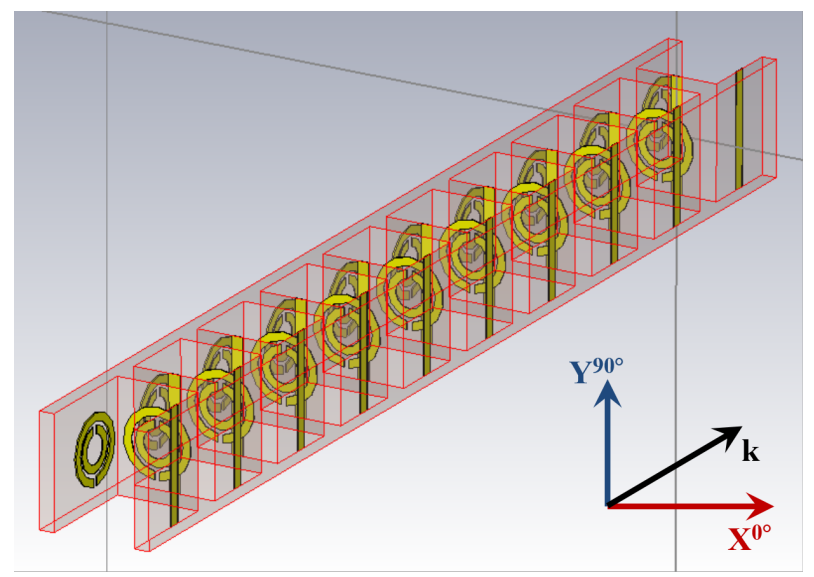

Figure 3.1: Unit cell setup used for computations with Microwave Studio@. Unit cell repetition boundary conditions were set along the $\mathrm{x}$ - and $\mathrm{y}$-axis and open boundaries set to the k-axis. Figure reproduced with permission of author [71].

evaluated for frequencies of $2 \mathrm{GHz}$ to $7 \mathrm{GHz}$ for the LFL configurations and for frequencies of $14 \mathrm{GHz}$ to $20 \mathrm{GHz}$ for the HFL configuration. In order to extract reflection and transmission from the modeling, reference cases with empty unit cells (air) and metal cells were run. By subtracting out the reference cases, and adding in phase correction terms due to the thickness of the lens, the reflected and transmitted electric fields were corrected. The metal calculations provided an ideal reflection standard, and the empty air case an ideal transmission standard. Together, the metal and air standards also defined the reference planes for the modeling. The following calculations generated correct reflection and transmission for both magnitude and phase.

$$
\mathbb{T}=\frac{T_{M T M} e^{i \theta_{T}}}{T_{A i r}} \quad, \text { where } \quad \theta_{T}=\frac{2 \pi \omega_{\text {all }} t_{M T M}}{c_{0}}-2 \pi
$$




$$
\begin{gathered}
\mathbb{R}=\left|\frac{R_{M T M}-R_{A i r}}{R_{A i r}}\right| e^{i \theta_{R}}, \text { where } \\
\theta_{R}=\varphi\left(\frac{R_{M T M}-R_{A i r}}{R_{\text {Air }}}\right)-\varphi\left(\frac{R_{\text {Metal }}-R_{\text {Air }}}{R_{\text {Air }}}\right)-\pi
\end{gathered}
$$

$\mathbb{T}$ denotes the corrected transmission, and $\mathbb{R}$ the corrected reflection. Transmission $(\mathbb{T})$ and reflection $(\mathbb{R})$ were complex values. The absolute value expression corresponds to the magnitude of the complex values and $\varphi$ the phase component of the complex values. The phase correction term in transmission $\theta_{T}$ was calculated over all modeled frequencies $\omega_{\text {all }}$. The thickness of the MTM lens was $t_{M T M}$, and the speed of light in vacuum was $c_{0}$. Phase correction in reflection $\theta_{R}$ took into account the phase difference between the MTM and air and the phase difference between metal and air; therefore $\theta_{R}$ and $\theta_{T}$ set the reference planes at the front and back interface of the MTM lens. $\mathbb{T}$ and $\mathbb{R}$ allowed for calculating a variety of electromagnetic properties, and were analogous to S-parameter microwave measurements.

\subsubsection{Modeling of Object Imaging}

In order to predict the imaging characteristics of the lenses, unit cell models (fig. 3.1) were utilized at the resonance frequency of the lenses. Due to extensive computational requirements of the unit cell model, calculations were simplified by inserting an ideal material with $n_{\text {eff }}=-1$. This decreased computational times significantly, as it idealized the system and removed computationally intensive resonance effects. The $n_{\text {eff }}=-1$ material was achieved by specifying a Drude resonance for both permittivity and permeability parameters in Microwave Studio's (c) material reference library. The simulation took into account the dimensions of the fabricated lens, feature sizes of the imaging samples, and lens to sample stand off distance. The modeling provided results for transmission and reflection modes.

In transmission mode, the modeling consisted of a plane wave source illuminating the object, a metal aperture, which was imaged by the lens and the $\vec{E}$ intensity was observed in the image plane. Open boundary conditions on all sides simulated a free space environment. 
(a) Transmission mode imaging

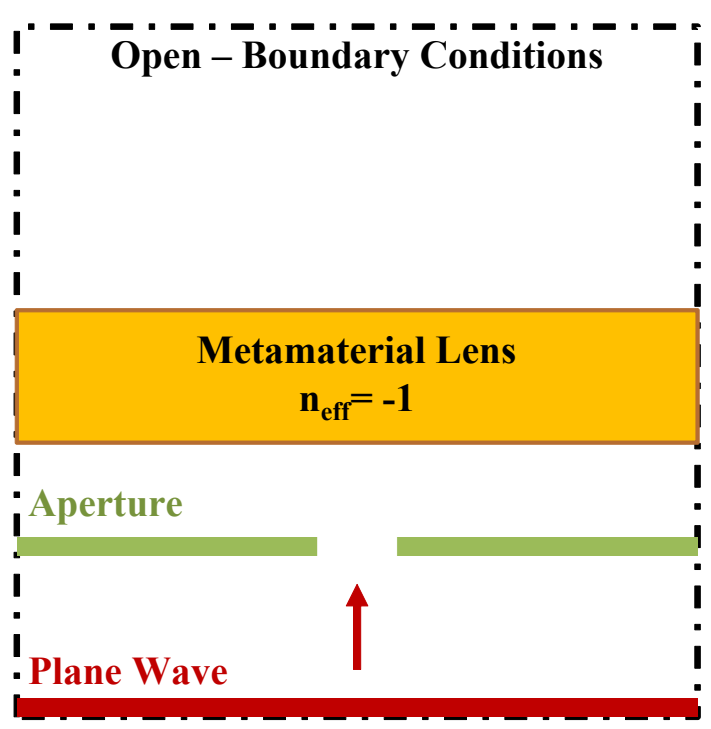

(b) Reflection mode imaging

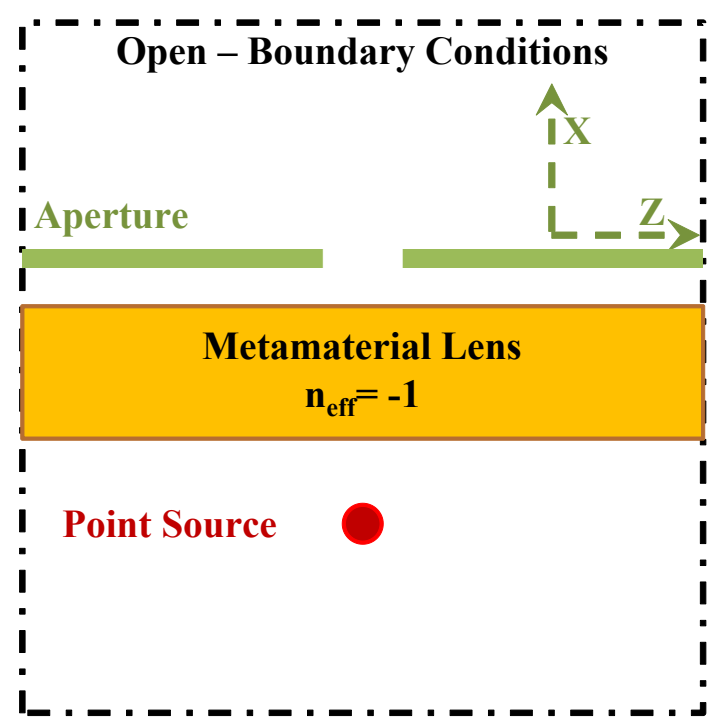

Figure 3.2: Modeling setup for transmission mode imaging (a) and reflection mode imaging (b). Open boundary conditions simulated free-space, the MTM lens was set to have $n_{\text {eff }}=-1$. (a) Incident plane wave illuminated an aperture sample which was imaged by the MTM lens in the focal plane. The results were obtained by solving for the $\vec{E}$ in the image plane. (b) A point source was focused onto the sample with the MTM lens. The aperture sample was moved along the X-Z plane and the $S_{11}$ reflection was calculated for the point source. (a) reproduced with permission of author [71].

To satisfy the imaging condition for a negative index lens, the object was placed at various distances while satisfying equation 2.12. Furthermore, simulations without the lens were performed to give a reference for near-field images of the samples.

For reflection mode imaging, a point source is placed in front of the dummy lens and is focused onto the image plane. An aperture sample is then swept through the focus and the point source's reflection $\left(S_{11}\right.$-parameter) was calculated as a function of aperture position. Several objects, including single as well as double slits of varying aperture opening and spacing were evaluated. The slit opening varied from $1 / 4$ to $2 \lambda$. For the double slit calculations, the slit opening was set to $\lambda / 2$, while the slit separation varied from $1 / 4$ to $1.5 \lambda$. The calculations were performed for both the LFL and the HFL. These evaluations gave baseline image performance under ideal conditions by using idealized MTM lenses, perfect sources, and detectors. 


\subsubsection{Design of Antenna}

For antennas in a single element configuration, the design was straightforward as the dipole and monopole antenna designs were easily fabricated and tuned with the help of a network analyzer. The antenna array configurations, however, required great care in design. Therefore Microwave Studio's (c) transient solver was used to design the five-element detector array. The overall design requirements were such that each element was spaced close to or within the imaging circle of the lens, while minimizing physical size and mutual coupling between elements.

(a) Single Element

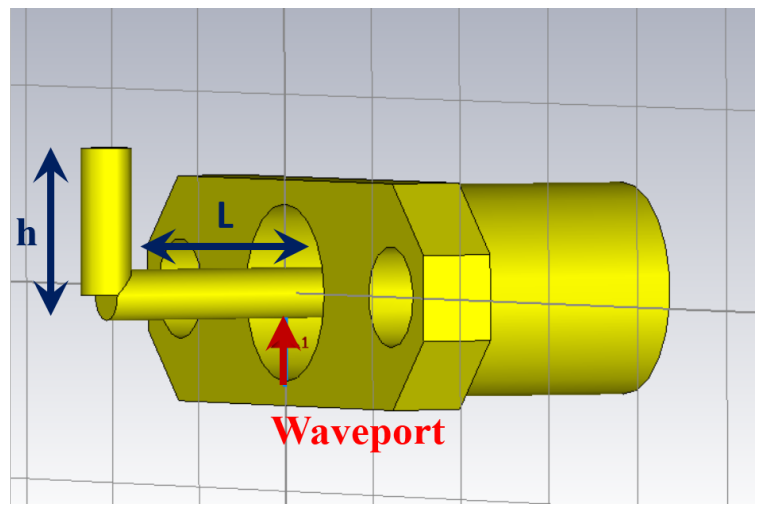

(b) Array

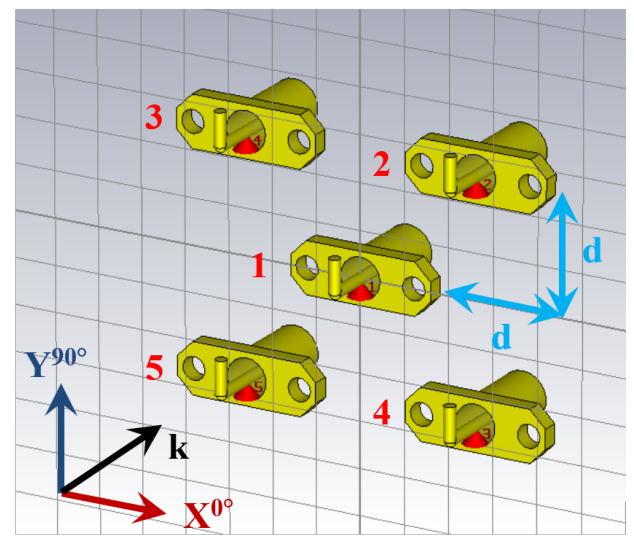

Figure 3.3: Diagram of the modeled antennas for the multi-detector array. (a) The antenna is optimized by varying the lead length $(\mathbf{L})$ and lead height $(\mathbf{h})$, red arrow shows the source polarization direction and waveport location. (b) Antenna spacing (d) in the array configuration is optimized. Mutual coupling between elements 1 through 5 was evaluated as well as the response to an incident plane wave (polarized along Y-axis and incidence along k-axis). Optimum configuration was calculated as $\mathbf{h}=3.7 \mathrm{~mm}, \mathbf{L}=5 \mathrm{~mm}$, and $\mathbf{d}=13 \mathrm{~mm}$.

Modeling of the elements consisted of each element in a X-formation with four elements on the corners of a square and one element in the center position, as shown in Fig.3.3. The antennas for each element were modeled with the SMA connector in mind and were based off of a monopole design due to space limitations. Open boundary conditions were fitted to the boundary of the modeling environment and the antenna was excited with a discrete waveport at the base of the antenna element. First, a single antenna element was modeled 
for its return-loss characteristics. The element's feature sizes were optimized for the HFL operating frequency. Subsequently, the relative spacing of the elements was varied and the mutual coupling between elements was retrieved from observing the crosstalk. Crosstalk was evaluated by exciting each element's discrete waveport one after the other and observing the S-parameters between the ports.

The overall array performance was then analyzed for its response to an incident plane wave. The ideal configuration, shown in Fig.3.3 a and b, was found to have $\mathbf{h}=3.7 \mathrm{~mm}$, $\mathbf{L}=5 \mathrm{~mm}$, and $\mathbf{d}=13 \mathrm{~mm}$. The fabricated multi-detector array was based on these values.

\subsection{Design and Fabrication}

\subsubsection{Metamaterial Lens}

The lenses were fabricated by tracing SRR and RE designs onto printed circuit board material and electroplating them with $\mathrm{Cu}$ to $30 \mu \mathrm{m}$ thickness (Fig.3.4). The LFLs were printed on rigid FR4 substrate which allowed for structuring the unit cell arrays into multiple configurations (Fig.3.5): a 2-D wine crate structure (2-D LFL), and 1-D structures (1-D LFL).

The HFL elements were printed on a Rogers Duroid $5880^{\mathrm{TM}}$ substrate and configured into the 2-D wine crate structure (2-D HFL). The MTM's resonance frequency is inherently related to the feature sizes of the resonant constituent elements (Fig.3.4), unit cell spacing and substrate material. Table 3.1 gives the specific features of each fabricated lens.
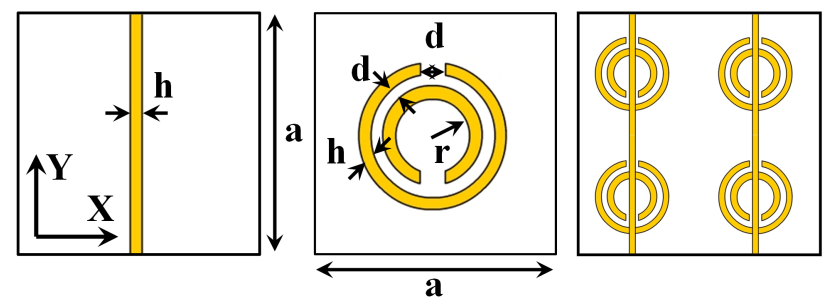

Figure 3.4: Feature sizes and arrangement of the MTM constituent elements: rod elements (left), SRRs (center), and unit cell arrangement (right). a is the size of the unit cell, $\mathrm{h}$ is the rod or ring width, $r$ is the inner radius of the $S R R$, and $d$ is the size of the split. Figure reproduced with permission of author [71]. 
(a)

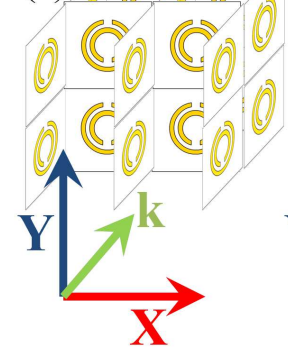

(b)

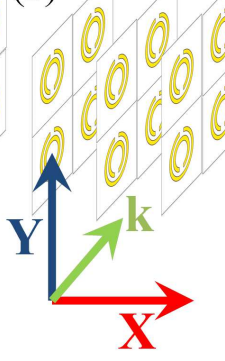

(c)

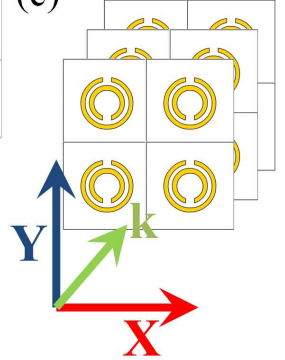

Figure 3.5: Diagram of different MTM unit cell configurations, (a) the 2-D wine crate structure for 2-D LFL and 2-D HFL, (b) the 1-D LFL, and (c) Crossboard LFL configuration. $\mathrm{X}$ and $\mathrm{Y}$ denote polarization axes. Typically, $\vec{E}$ of incoming $\mathrm{MW}$ is polarized along the $\mathrm{Y}$-axis and $\vec{H}$ is along the X-axis. $\vec{k}$ denotes the propagation direction of incident MW.

\subsubsection{Antenna and Detectors}

The imaging configurations require a source antenna for MW illumination and receive antennas coupled to diode detectors for signal detection. The antennas were designed and fabricated in house and were based on simple dipole and monopole antennas. In the case of the LFL experiment, the receive antenna was a printed circuit board dipole antenna with an integrated balun. All other antennas were made of free standing wires soldered to SMA (SubMinature version A) feeds. The antennas initially had wire lead lengths according to the standard half wavelength dipole or quarter wavelength monopole architecture. Antenna tuning was further refined with the help of a network analyzer by incrementally shortening the wire leads. The network analyzer (Agilent PNA E8362B) was calibrated with a short-open-load S-parameter one-port calibration to the ends of the cable. With the SMA feed of the antenna structure attached to the calibrated cable, a $S_{11}$ return loss measurement was performed as a function of frequency. The $S_{11}$ return loss frequency response was observed and the antenna leads were incrementally shortened until the minimum of the $S_{11}$ response was less than $-10 \mathrm{~dB}$ and aligned with the MTM's resonance frequency.

Six zero-bias detector diodes were purchased for the imaging study. The detectors were selected for their specifications which met the MTM operating frequency requirements, minimum power detection capabilities, and availability. The selected detectors were one 
Table 3.1: Constituent element feature sizes and substrate design values, as corresponding to fig. 3.4. Dimensions of the lenses are given in \# of unit cells in width $\mathrm{W}$, height $\mathrm{H}$, and gap D. Substrate information gives the permittivity of the substrate $\epsilon$, loss tangent $\tan \delta$, thickness of the boards $\mathrm{t}$, and copper $(\mathrm{Cu})$ trace thickness.

\begin{tabular}{|c|c|c|c|c|c|c|}
\hline $\begin{array}{l}\text { MTM } \\
\text { Lens }\end{array}$ & Substrate & $\begin{array}{c}\text { \# unit cells } \\
(\mathrm{W}, \mathrm{H}, \mathrm{D})\end{array}$ & $\begin{array}{c}\mathrm{a} \\
(\mathrm{mm})\end{array}$ & $\begin{array}{c}\mathrm{r} \\
(\mathrm{mm})\end{array}$ & $\begin{array}{c}\mathrm{h} \\
(\mathrm{mm})\end{array}$ & $\begin{array}{c}\mathrm{d} \\
(\mathrm{mm})\end{array}$ \\
\hline LFL & $\begin{array}{l}\text { FR4 } \\
\epsilon=4.4 \\
\tan \delta=0.02 \\
t_{s u b}=1.8 \mathrm{~mm} \\
t_{C u}=30 \mu \mathrm{m}\end{array}$ & $\begin{array}{l}W=40 \\
H=20 \\
D=10\end{array}$ & 9.3 & 1.6 & 0.9 & 0.2 \\
\hline HFL & $\begin{array}{l}\text { Duroid } 5880 \\
\epsilon=2.2 \\
\tan \delta=0.0009 \\
t_{\text {sub }}=1 \mathrm{~mm} \\
t_{C u}=30 \mu \mathrm{m}\end{array}$ & $\begin{aligned} W & =100 \\
H & =60 \\
D & =10\end{aligned}$ & 3.8 & 0.7 & 0.3 & 0.2 \\
\hline
\end{tabular}

Anritsu 70KA50 for single detector imaging and five Fairview Microwave SMD0218 for multidetector imaging. Their voltage output versus microwave power in response was amplified with op-amp circuits. The op-amp (Texas Instruments LM321) used a power supply set to $\pm 5 V$ (Hewlett-Packard 6236B Triple Output Power Supply), and gain $(G)$ was set via resistive loads $\left(G=\frac{\mathbf{R}_{1}}{\mathbf{R}_{0}}\right)$. The amplification circuit shown in Fig. 3.6 was set up in an inverting configuration, as the diode detectors exhibited negative polarity. The amplification factors were chosen to give voltage output response between $\pm 5 \mathrm{~V}$. The circuitry was mounted on a bread board and fed the amplified detector voltage to the data acquisition system (National Instruments USB-6008). The detector system was calibrated as outlined in the appendix. Computational time domain simulations further optimized antenna design and detector placement for the multi-detector setup.

\subsubsection{Samples for Imaging}

Samples were fabricated from $1.63 \mathrm{~mm}$ thick sheet metal and FR4 glass fiber. The test articles consisted of single and dual apertures, and single and dual holes. The aperture 


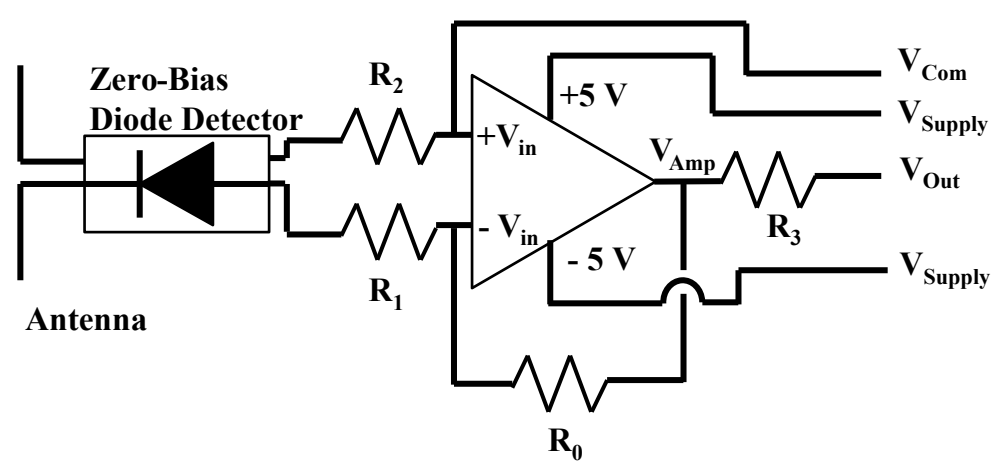

Figure 3.6: Detector circuit design: the antenna is connected to the commercially purchased zero-bias diode detector (Model: Anritsu 70KA50 or Fairview Microwave SMD0218) and the amplification circuit comprised of four resistors $\left(\mathbf{R}_{\mathbf{0}}\right.$ to $\left.\mathbf{R}_{\mathbf{3}}\right)$ and the Op-Amp (TI LM321). For the single detector study the resistors were set to $\mathbf{R}_{\mathbf{0}}=12 \times 10^{6} \Omega \pm 5 \%, \mathbf{R}_{\mathbf{1}}=120 \Omega \pm 5 \%, \mathbf{R}_{\mathbf{2}}=$ $120 \Omega \pm 5 \%$, and $\mathbf{R}_{\mathbf{4}}=47 \Omega \pm 5 \%$ giving an amplification factor of $G=1 \times 10^{5} \pm 7.1 \%$. For the multi-detector setup, the resistors were $\mathbf{R}_{\mathbf{0}}=12 \times 10^{6} \Omega \pm 5 \%, \mathbf{R}_{\mathbf{1}}=56 \Omega \pm 5 \%, \mathbf{R}_{\mathbf{2}}=$ $68 \Omega \pm 5 \%$, and $\mathbf{R}_{\mathbf{4}}=47 \Omega \pm 5 \%$ giving an amplification factor of $G=2.14 \times 10^{5} \pm 7.1 \%$.

samples were cut with the help of NASA - Langley Research Center's water-jet fabrication facility in order to ensure accurate cuts and tolerance. Their parameters are outlined in table 3.2. The sample features were selected such that they could be tested for image resolution and imaging sensitivity. In addition to the samples with features, several blank samples were cut to size so as to provide a reference for image processing. Blind aperture and hole samples were made by covering and backing the samples with a $30.5 \mathrm{~cm} \times 30.5 \mathrm{~cm}$ cut out of the FR4 stock material. Other image targets were provided by what was on hand and fabricated from the FR4 stock, aluminum tape, and sheet metal. 
Table 3.2: Sample feature sizes, as corresponding to Fig.3.7. Dimensions of the features are given in $\mathrm{mm}$ and in fractions of wavelength $(\lambda)$.

\begin{tabular}{|c|c|c|c|}
\hline Sample & Substrate & $\begin{array}{l}\text { Feature size } \\
(\mathrm{mm}(\lambda))\end{array}$ & $\begin{array}{l}\text { Separation } \\
(\mathrm{mm}(\lambda))\end{array}$ \\
\hline \multirow{3}{*}{ Single Aperture } & \multirow{3}{*}{ Metal \& FR4 } & $18(\lambda)$ & $\mathrm{N} / \mathrm{A}$ \\
\hline & & $9(0.5 \lambda)$ & $\mathrm{N} / \mathrm{A}$ \\
\hline & & $4.5(0.25 \lambda)$ & $\mathrm{N} / \mathrm{A}$ \\
\hline \multirow{4}{*}{ Dual Apertures } & \multirow{4}{*}{ Metal \& FR4 } & $9(0.5 \lambda)$ & $36(2 \lambda)$ \\
\hline & & $9(0.5 \lambda)$ & $27(1.5 \lambda)$ \\
\hline & & $9(0.5 \lambda)$ & $18(\lambda)$ \\
\hline & & $9(0.5 \lambda)$ & $13.5(0.75 \lambda)$ \\
\hline \multirow{5}{*}{ Single Hole } & \multirow{5}{*}{ FR4 } & $5.9(0.33 \lambda)$ & $\mathrm{N} / \mathrm{A}$ \\
\hline & & $4.6(0.26 \lambda)$ & $\mathrm{N} / \mathrm{A}$ \\
\hline & & $3.3(0.18 \lambda)$ & $\mathrm{N} / \mathrm{A}$ \\
\hline & & $2.1(0.12 \lambda)$ & $\mathrm{N} / \mathrm{A}$ \\
\hline & & $1(0.06 \lambda)$ & $\mathrm{N} / \mathrm{A}$ \\
\hline \multirow{5}{*}{ Dual Hole } & \multirow{5}{*}{ FR4 } & $9(0.5 \lambda)$ & $27(1.5 \lambda)$ \\
\hline & & $9(0.5 \lambda)$ & $18(\lambda)$ \\
\hline & & $9(0.5 \lambda)$ & $15(0.83 \lambda)$ \\
\hline & & $9(0.5 \lambda)$ & $12(0.67 \lambda)$ \\
\hline & & $9(0.5 \lambda)$ & $10(0.56 \lambda)$ \\
\hline
\end{tabular}

(a) Dual Aperture Sample

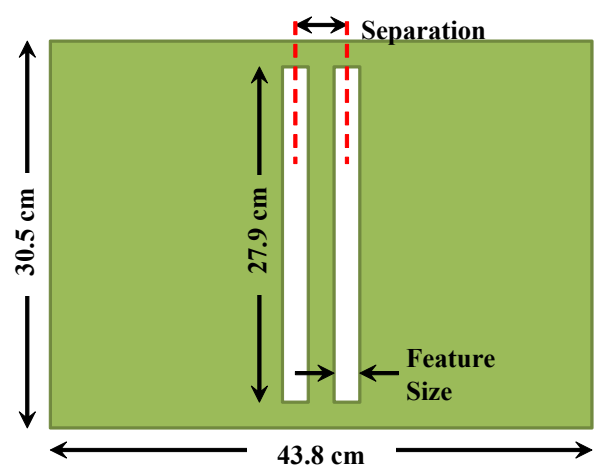

(b) Dual Hole Sample

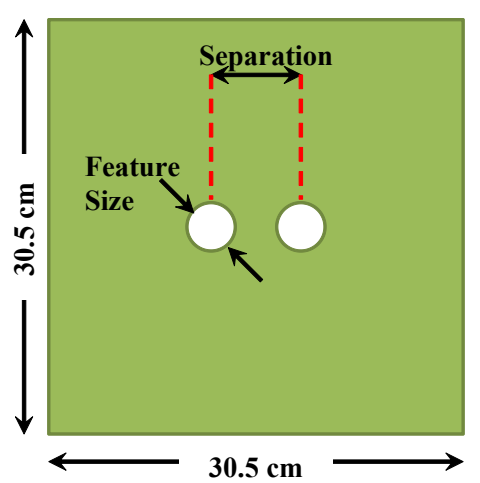

Figure 3.7: Schematic of dual aperture and dual hole samples. The samples were fabricated from metal or FR4 of $1.63 \mathrm{~mm}$ thickness. (a) Dual apertures are cut via water-jet, (b) dual holes are drilled. Material, separation, and feature sizes are varied according to table 3.2. Single aperture and single holes samples have the same dimensions as the dual aperture and dual hole samples. Figure reproduced with permission of author [71]. 
Chapter 3 | Metamaterial Lens Modeling, Design, Fabrication, and Characterization 32

\subsection{Characterization of Metamaterial Lens Properties}

The MTM lenses were characterized for their EM properties by measuring the four Sparameters $\left(S_{11}, S_{21}, S_{12}, S_{22}\right)$. This gave insight into reflection, transmission, and resonant characteristics as well as their indices of refraction. The experiment was performed in a modular semi-anechoic environment capable of free space S-parameter and polarization rotation measurements. The measurement chamber at NASA Langley's Electromagnetics and Sensors Branch consisted of a sample stage surrounded with absorber on both sides as well as cone-type absorber on front and back walls, and floor. A transmission, reflection, and line (TRL) calibration procedure was adapted for free space in order to account for measurement inaccuracies in the system. Further baseline correction and time domain gating techniques minimized post-calibration errors. The exact calibration procedure is presented in appendix A. The system included a precision network analyzer (Agilent PNA E8362B or PNA-X 5242A), matched linear polarized horn antennas and phase stable semi-rigid coaxial cables. A schematic of the experimental setup is shown in Fig. 3.8. The LFL configurations were measured with broadband $2 \mathrm{GHz}$ to $18 \mathrm{GHz}$ Cobham Sensor Systems H-1498 horn antennas, whereas the HFL was analyzed with broadband $12 \mathrm{GHz}$ to $18 \mathrm{GHz}$ Waveline 799 horn antennas.

For each configuration, the antennas were placed in the far-field with respect to the lens, at $40 \mathrm{~cm}$ from the lens. This ensured that the MTMs electromagnetic properties were investigated with incident plane waves. The plane wave configuration was important so as not to introduce measurement errors due to refraction. The far-field position of the horn antennas was derived from the equation below,

$$
\mathcal{R}_{\text {farfield }}=\frac{2 \varnothing^{2}}{\lambda}
$$

where $\varnothing$ is the diameter of the horn antenna and $\lambda$ is the wavelength. After calibration, the S-parameters were measured for the LFLs from $3 \mathrm{GHz}$ to $6 \mathrm{GHz}$, in steps of $1.5 \mathrm{MHz}$. The 


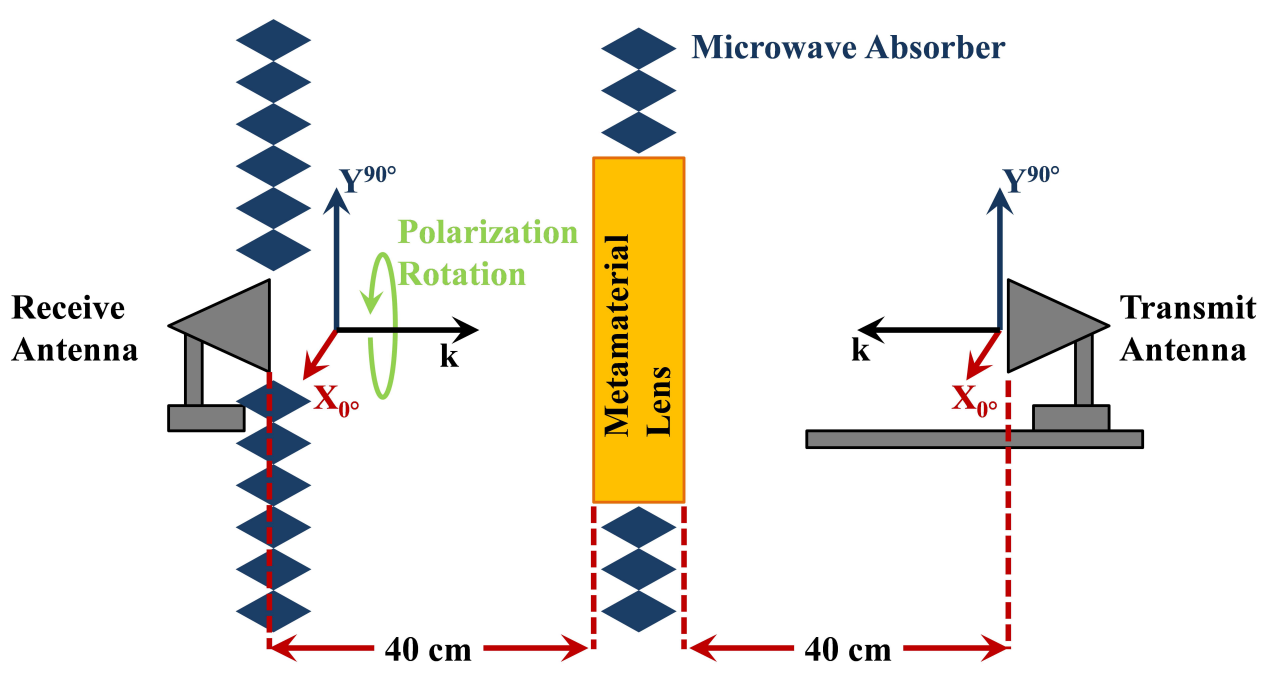

Figure 3.8: Experimental setup for the characterization of the electromagnetic properties and polarization characteristics of the MTM lens. Horn antennas are in parallel polarization with respect to each other, the incident $\vec{E}$ is to be parallel to the rods of the MTM lenses and along the y-axis. Both horn antennas are set equidistant, in the far-field, from the MTM lens. Absorber shielded the measurement environment from noise, isolated the transmit and receive horn antennas, and made sure that the received energy had passed through the MTM. Figure reproduced with permission of author [71].

$S_{11}, S_{12}, S_{21}$, and $S_{22}$ data were recorded for magnitude and phase, where the reflection and transmission coefficients were derived from $S_{11}$ and $S_{21}$ measurements. Similarly, the HFL was investigated from $14 \mathrm{GHz}$ to $18 \mathrm{GHz}$, in steps of $1.5 \mathrm{MHz}$.

This research investigated the MTM's susceptibility to the polarization of the incident MW. In optics, the polarization state of the incident radiation is the basis for many sensors and image enhancement techniques. Therefore, it is advantageous to study the effect of the 2-D and 1-D MTM LFLs on the transmitted polarization state as a function of incident polarization angle. This work resulted in two publications and a presentation [72, 73]. The study compared quantitative predictions with the measured polarization properties of experimentally realizable structures. These properties were investigated by adapting the S-parameter measurement system (Fig. 3.8) for a polarization experiment. The transmit and receive horn antennas were placed on axial rotation stages, rotating around the direction of MW propagation. The measurements were calibrated with both source and receive horn antennas in parallel polarization with respect to each other. Two different measurements were 
made. In the first measurement the source and receive horns were in parallel polarization, while the incident polarization angle was changed with respect to the lens. This is analogous to rotating the MTM lens with respect to $\vec{E}$. In the second measurement, the incident polarization was fixed while the receive horn was rotated to analyze the transmitted $\vec{E}$. Measurements were taken for the LFL in 2-D, 1-D and Crossboard configuration (Fig. 3.5).

\subsection{Imaging Experimental Setup}

The subwavelength focusing capabilities of the 1-D LFL, 2-D LFL, and the HFL have been previously demonstrated $[42,69]$. In this study, the inherent lensing properties of the SRR and RE MTMs were applied to highlight their imaging capabilities. In practice, it is very difficult to obtain MTM lenses with theoretically idealized $n_{e f f}=-1+i 0$ conditions. The best image quality in terms of resolution was realized at negative indices of refraction close to the theoretical ideal. The imaging properties were investigated in two separate setups, a back-illuminated transmission mode and a front-illuminated reflection mode.

\subsubsection{Transmission Mode Imaging}

The transmission imaging setup (Fig. 3.9) consisted of a source, a detector, a three axis translation stage, a data acquisition system, and the MTM lens. A semi-anechoic measurement environment encased the setup with absorber walls isolating the source from the detector side. This ensured that the received energy had passed through the lens.

MWs were generated with a HP 8341B Synthesized Sweeper in conjunction with a $+30 \mathrm{~dB}$ HP 8349B Microwave Amplifier. The source was set to continuous wave mode, as the MTM lenses operate at a single frequency and steady signal. A horn antenna was used as the source, in the far-field $(40 \mathrm{~cm})$ with respect to the test sample. The MTM lens then imaged the illuminated sample. Sample positioning was within the lensing condition (see Fig. 2.3a) of 
the MTM lens. In a typical setup the sample to metamaterial lens distance, called the front focal length $\left(f_{1}\right)$, was set to the length of half the thickness of the lens $\left(\frac{t}{2}\right)$.

To observe the image, a commercially available detector was attached to the Isel DaVinci Series 1012 three axis positioner. The detector system swept in the image plane of the lens. The National Instruments USB 6008 data acquisition system in conjunction with LabView $^{\mathrm{TM}}$ software recorded a transmitted voltage for each position of the detector. The detected voltage was interrogated at $10 \mathrm{kHz}$ over a period of 0.2 seconds and filtered for interfering signals with a bandpass algorithm in LabView. These conditions maintained a reasonable time step between measurements, while acquiring adequate number of voltage measurements for noise filtering. The voltage was then converted to a received power reading using calibrated power-in to voltage-out curves.

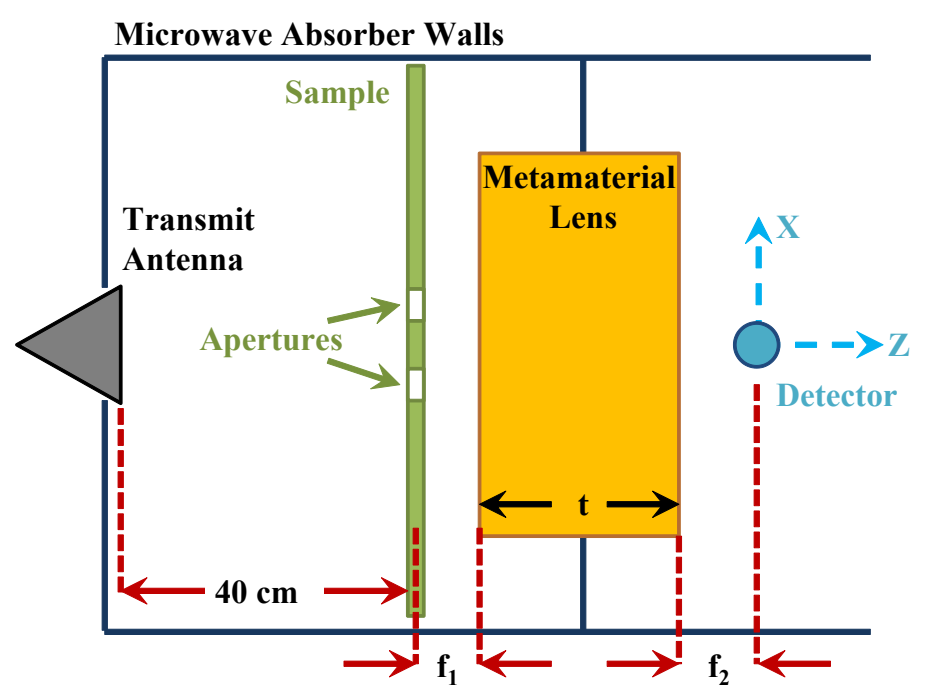

Figure 3.9: Experimental setup for transmission mode imaging. The detector traces out the object image, step by step in the $\mathrm{x}-\mathrm{z}$ plane.

The objects under investigation were sheet metal samples with aperture cutouts. The samples were sized larger than the lenses. This minimized errant MW signals in the image plane and ensured that the image was only of the aperture. The MW signal that passed through the apertures was interrogated with and without the lens. The no-lens condition provided for a near-field reference image. Furthermore, images obtained from the direct 
Chapter 3 | Metamaterial Lens Modeling, Design, Fabrication, and Characterization 36

near-field measurements and the MTM lens system were compared to modeling. The results were investigated for image resolution.

\section{Low Frequency Lens}

The 2-D LFL lens was investigated for its focusing properties by evaluating its imaging capability with a dipole source. In this setup, instead of using an aperture, the system was configured to directly image a dipole antenna. This antenna acted as the equivalent of a point source. From this, the focus spot would dictate the resolution. For investigating aperture objects, the 1-D LFL was used as it had a higher transmission coefficient compared to the 2-D LFL at resonance. The AEL horn antenna acted as the source and the frequency was set to $3.6575 \mathrm{GHz}$. The Linear Technologies LTC 5582 linear detector on a 1528A Demo Circuit detected the MW signal. Two single aperture samples made of sheet metal with feature sizes $\lambda / 2$ and $\lambda$ were measured.

\section{High Frequency Lens}

For the HFL, the detector was changed to a $2 \mathrm{GHz}$ to $18 \mathrm{GHz}$ Anritsu 70KA50 SMC packaged detector and the source horn was switched to the Waveline 799s. The source operated at a frequency of $16.60 \mathrm{GHz}$. Sheet metal apertures outlined in table 3.2 were imaged and the image resolution was investigated.

\subsubsection{Reflection Mode Imaging}

For real world applications, transmission mode imaging has limitations, as it requires independent source and detector placement. The reflection mode imaging setup places the source and detector on the same side with respect to the object (Fig. 3.10). A dipole antenna source was situated between the lens and the sample and operated at a frequency of $16.672 \mathrm{GHz}$. The lens was the 2-D HFL and the detector was positioned in the image plane. The three axis positioner scanned the object in front of the source. In the experiment the transmit 
antenna to lens distance $(\mathrm{m})$ was approximately $2 \mathrm{~mm}$. The back-focal length $\left(f_{2}\right)$ was $8 \mathrm{~mm}$ for the single detector study with a front focal length $\left(f_{1}\right)$ of $23 \mathrm{~mm}$.

The imaging samples discussed in table 3.2 were measured. In addition, a high-contrast object was used to highlight the imaging capabilities. The high contrast object consisted of thin letters of aluminum spelling "UVA." The three letters were spaced in a $150 \mathrm{~mm}$ by $50 \mathrm{~mm}$ area. The letter traces were $9 \mathrm{~mm}$ wide and the smallest separation between letters was $14 \mathrm{~mm}$. The letters were cut out of aluminum tape. The sample was evaluated by scanning in the $\mathrm{x}-\mathrm{y}$ plane at the focus, which was located at $23 \mathrm{~mm}$ from the front surface of the lens.

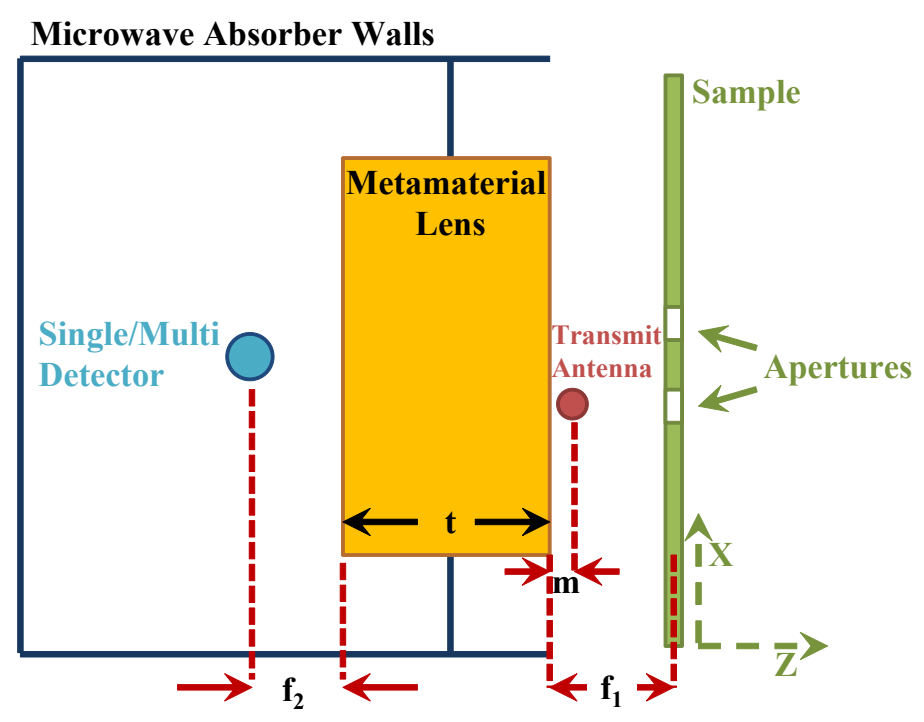

Figure 3.10: Experimental setup for reflection mode imaging. The source and detector interrogate the object from the same side of the sample. The image is observed by stepping the object in the $\mathrm{x}-\mathrm{z}$ plane. 2-D images are retrieved by stepping the object in the $\mathrm{x}-\mathrm{y}$ plane with the z- position locked at the focal plane. Figure reproduced with permission of author [71].

Application of the imaging technique to hidden objects was crucial in providing a proof of concept for the envisioned MTM based MW imaging system. Several samples in the study were covered to provide "blind" objects. This aided in determining the resolution and sensitivity for detecting buried objects. 
Chapter 3 | Metamaterial Lens Modeling, Design, Fabrication, and Characterization 38

\section{Imaging with polarized detectors}

Polarization plays a great role in imaging applications and is commonly used for contrast enhancement. Co-polarized and cross-polarized images are particularly helpful in image reconstruction of objects. Recent literature shows the applicability of polarization dependent image enhancement of objects in the presence of high noise backgrounds [74] and image enhancement of hidden objects [75]. However, this technique has not been applied to MTMs based imaging. Similarly, through utilization of polarization properties of incident microwave radiation and detected radiation, the MTM lens based imaging system can be further improved. The polarization properties of the MTM lens was evaluated by rotating the polarization of the receive antenna. Measurements were performed with the transmit antenna polarized along the $y$-axis $\left(90^{\circ}\right)$ and receive antenna polarization axis along the $y$-axis $\left(90^{\circ}\right)$ (which is the co-polarized image), at $\pm 45^{\circ}$ (which is the partially cross-polarized image), and along the $\mathrm{x}$-axis $\left(0^{\circ}\right)$ (which is the cross-polarized image).

\subsubsection{Multi-Point Array Imaging}

The MTM imaging capabilities have been demonstrated with single point-to-point detection. This approach is time consuming as well as impractical for commercial applications. Therefore, the previously established single point imaging technique was improved by making use of a detector array. The array was capable of interrogating the image plane at several spots simultaneously, which lowered the image capture time. Furthermore, the information from multiple detectors could be averaged for image enhancement.

A total of five detectors were integrated, where each individual detector was linked to a single antenna so as to represent a single image pixel (see fig. 3.11a and 3.11b). The array was setup in an X-configuration and calibrated as outlined in Appendix B. To minimize noise in the detector system, voltage measurements were taken at $2 \mathrm{kHz}$ over a period of 0.5 seconds. Their signal was then filtered with a bandpass algorithm in LabView. Of the filtered signal 500 data points were averaged to arrive at the detected voltage. The voltage 
was then converted into a power reading using calibration values. This aggregate information was fed into the data acquisition system, where the received detector information was indexed as a function of position of the object and subsequently the image was extracted with Matlab.

The challenge in implementing the multiple detectors was in positioning the array in such a way that all of the receive antennas were within or close to the focal spot of the lens. Furthermore, the individual array elements were aligned to be in the $\mathrm{x}-\mathrm{y}$ image plane with a back focal length $\left(f_{2}\right)$ of $14 \mathrm{~mm}$ (see fig. 3.11c). Source antenna to lens standoff distance $(\mathrm{m})$ remained unchanged at $2 \mathrm{~mm}$. Object positioning was determined by sweeping the object in the $\mathrm{x}-\mathrm{z}$ plane to observe a sharp image. 2-D images were then taken by sweeping the object in the $\mathrm{x}-\mathrm{y}$ plane with the front focal length $\left(f_{1}\right)$ at the ideal z-position. Depending on the sample, $f_{1}$ was between $20 \mathrm{~mm}$ and $25 \mathrm{~mm}$.

The images were evaluated to obtain the MTM imaging system's resolution and sensitivity capabilities. In addition to comparing the performance of the system to single detector images, the multi-point array imaging system was analyzed for image enhancement through adding the information of five detectors into a single image as well as reducing image capture times through sub-sampling. 
(a) Multi-Detector Array Front

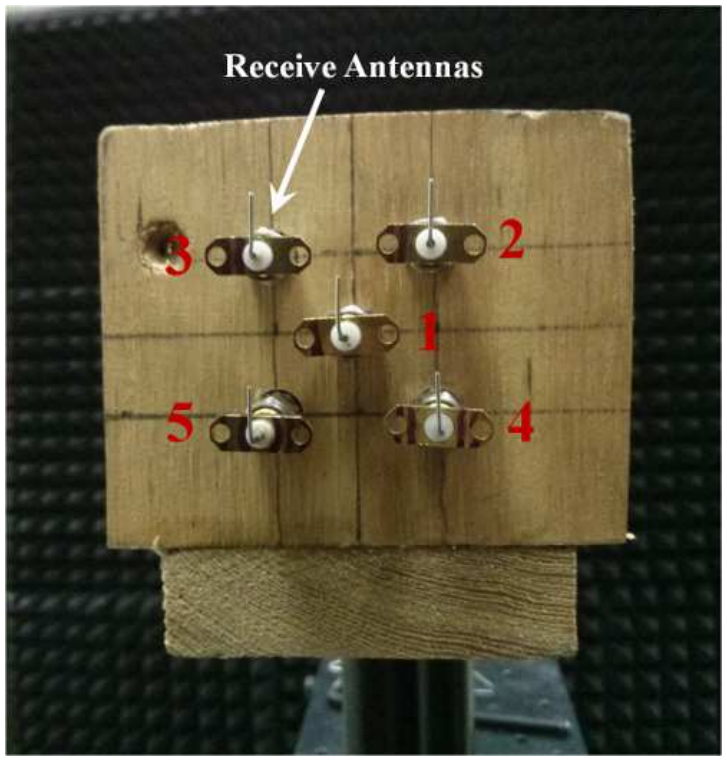

(b) Multi-Detector Array Back

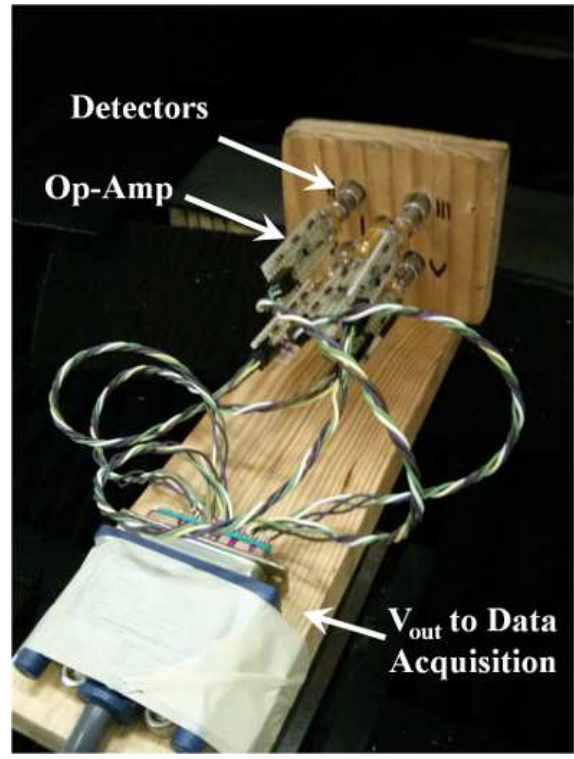

(c) Multi-Detector Array Imaging configuration

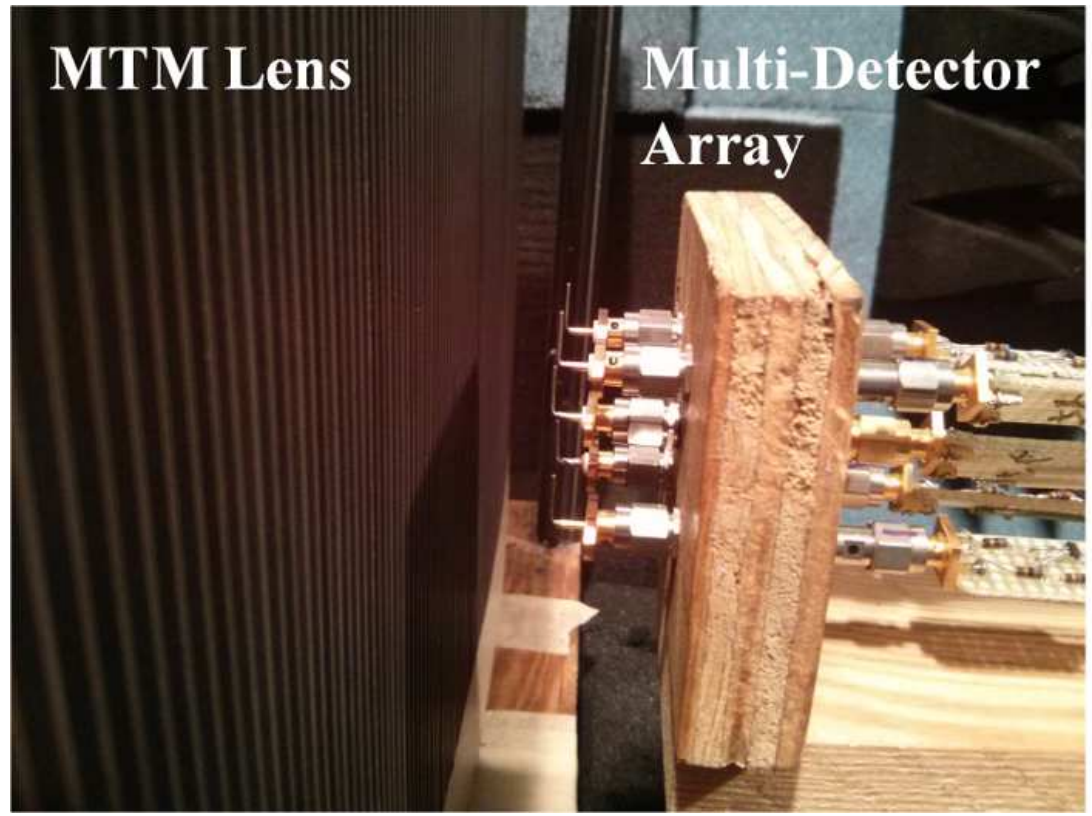

Figure 3.11: The fabricated multi-detector array. 3.11a shows the location of detectors 1 through 5. Detector spacing is the same as specified from modeling. 3.11b shows the detector connected to op-amp circuitry and voltage out to the data acquisition system. 3.11c shows the detector array in the imaging configuration with the HFL. 


\section{Chapter 4: Metamaterial Lens}

\section{Electromagnetic Properties Results}

\section{and Discussion}

The electromagnetic properties of the metamaterial lenses dictate the imaging performance of the system. The transmission and reflection profiles directly affects the resolution of the lens, as the resolution is heavily dependent on the absorption of the lens. Furthermore, the reflection and transmission coefficients are used to calculate the effective index of refraction of the lenses as a function of frequency, with the goal of finding an index of refraction as close as possible to the ideal $n_{\text {eff }}=-1+i 0$ case. Additionally, in optics, the control of polarization properties is the basis for many sensor applications. Similarly, the control over the polarization properties of MTM elements can lead to novel sensor and imaging applications. Therefore, this chapter compares the measured electromagnetic properties to modeled predictions. From this data, the optimum frequency for the imaging system was chosen.

\subsection{Transmission and Reflection}

As is shown in fig. 4.1, the transmission and reflection response of these metamaterial lenses exhibit similar behavior. They exhibited a low transmission region preceding the resonance region as well as following the resonance region until the onset of a passband. The reflection profile was similar as well, as near perfect reflection was observed until the resonance region, at which point the reflection was suppressed. Past the resonance region, reflection remained high and then fell off with the increase in frequency. 
(a) 2-D Low Frequency Lens

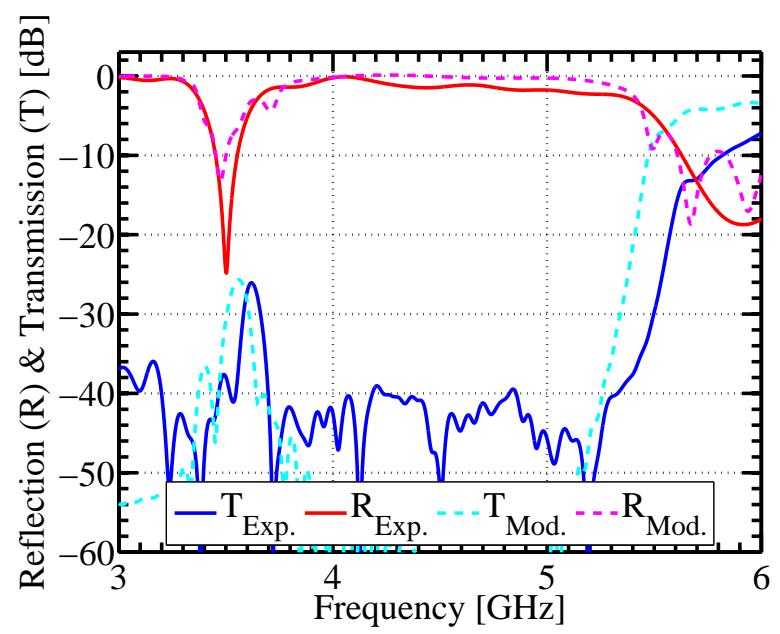

(b) 2-D High Frequency Lens

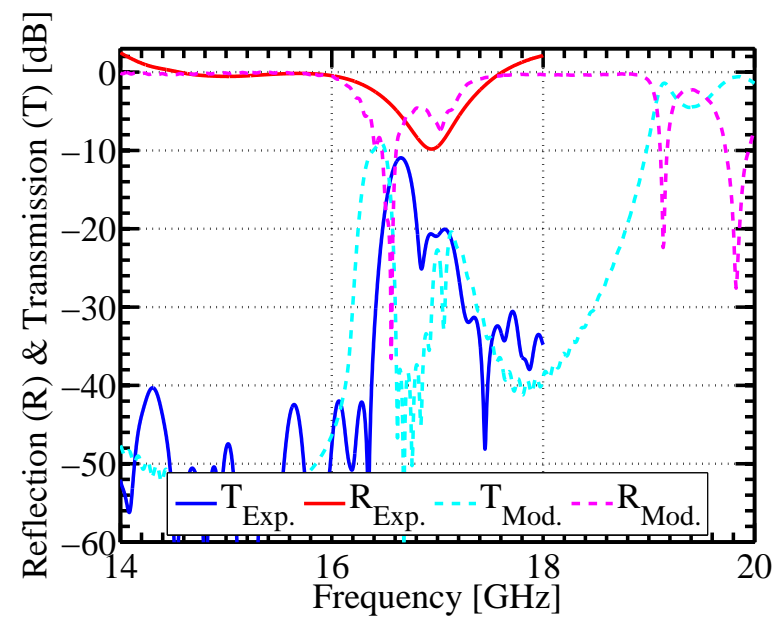

(c) Teflon Reference Sample

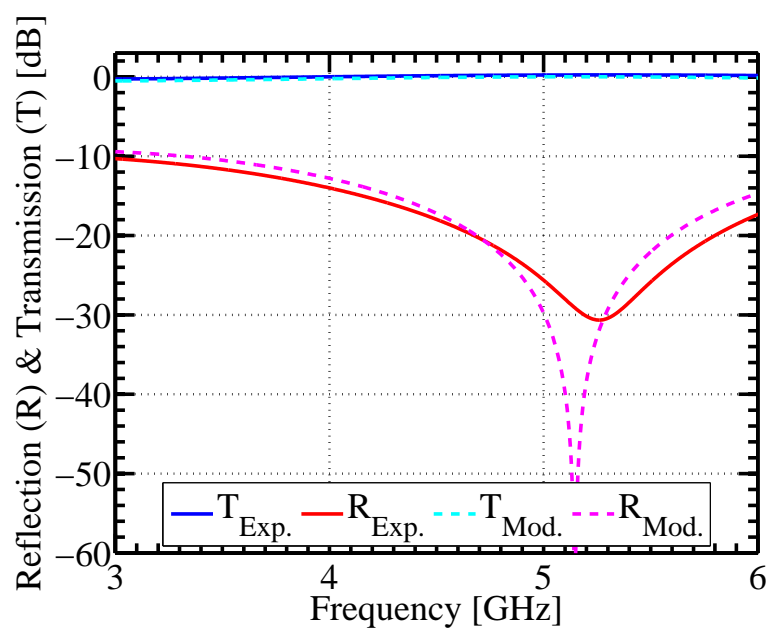

Figure 4.1: Transmission (T) and reflection (R) coefficients of the MTM lenses and for a Teflon reference sample. Experimental Data (Exp.) is compared to modeled results (Mod.). The modeled results were based on the as specified permittivity, and feature size dimension. The experimental results match well with modeling. The reflection and transmission values above $0 \mathrm{~dB}$ are due to the Fourier transforms inherent to the time domain gating technique used during calibration. (b) reproduced with permission of author [71]. 
(a) 2-D Low Frequency Lens Refl. Phase

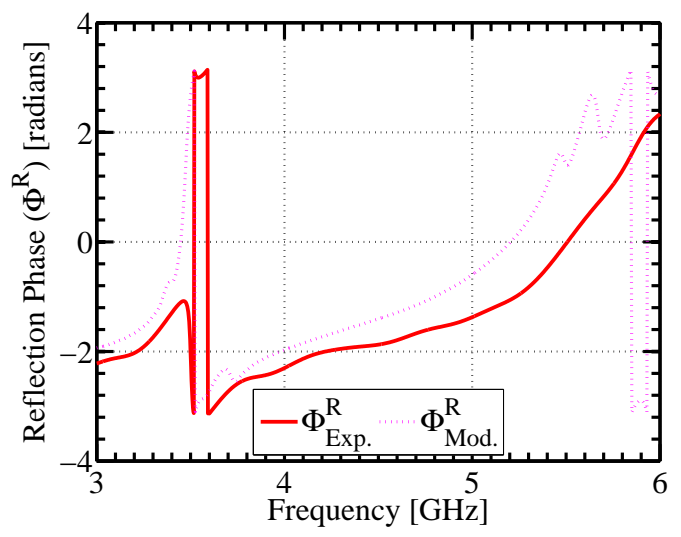

(b) 2-D High Frequency Lens Refl. Phase

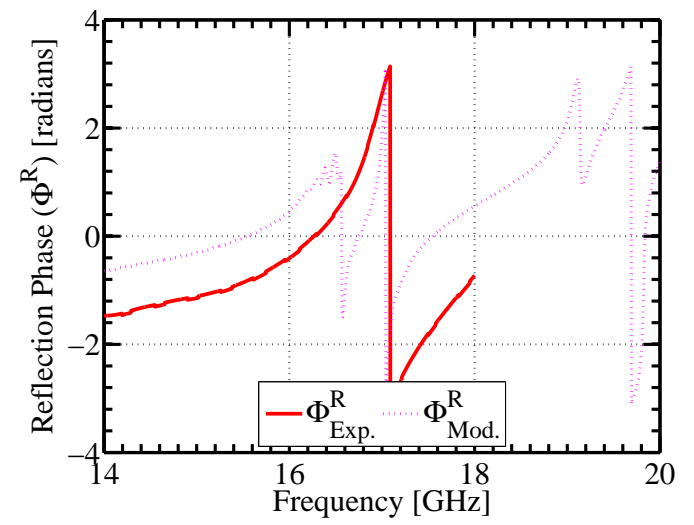

(c) Teflon Reference Refl. Phase

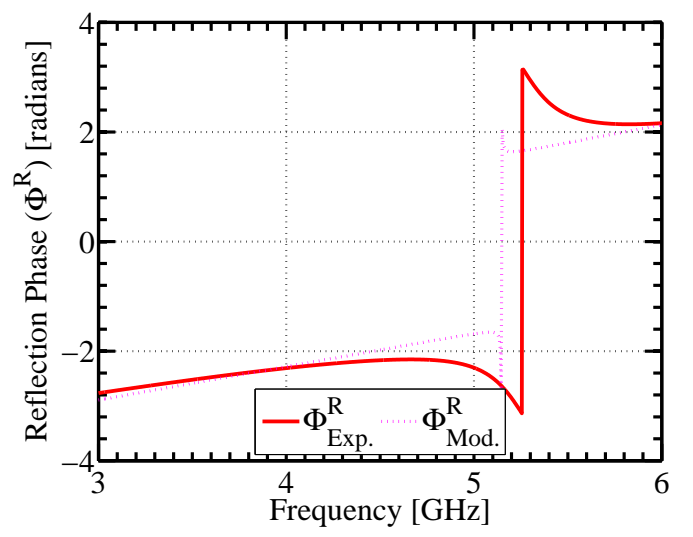

Figure 4.2: Reflection (R) phase $(\Phi)$ information of the MTM lenses and for a Teflon reference sample. Experimental data (Exp.) is compared to modeled results (Mod.). The modeled results were based on the as specified permittivity, and MTM feature size dimensions. The experimental results matched well with modeling.

The phase information for the 2-D MTM lenses and the Teflon reference sample are presented in fig. 4.2 and fig. 4.3. The measured phase from reflection matches the modeled predictions well, there are small frequency mismatches at the phase wrapping points when the phase cycles from $-\pi$ to $+\pi$. For transmitted phase information multiple phase wrappings correlated with very low transmission regions (below $\approx-45 \mathrm{~dB}$ ) which are due to forbidden band regions inherent to the MTM (see fig. 4.3). Phase wrappings also correlated with oscillations in modeled transmission (fig. $4.3 \mathrm{c}$ and fig. $4.3 \mathrm{~d}$ ), as well as measurements near the noise floor (fig. 4.3a and fig. 4.3b). The large change in phase at the MTM resonance was due 
(a) Exp. 2-D LFL Trans. Phase

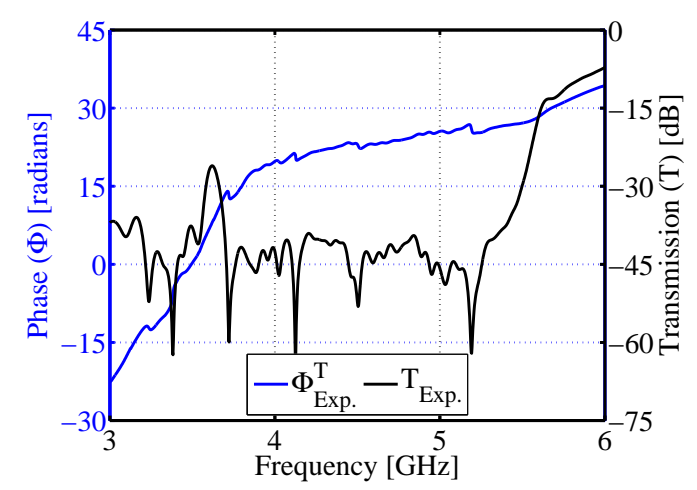

(c) Mod. 2-D LFL Trans. Phase

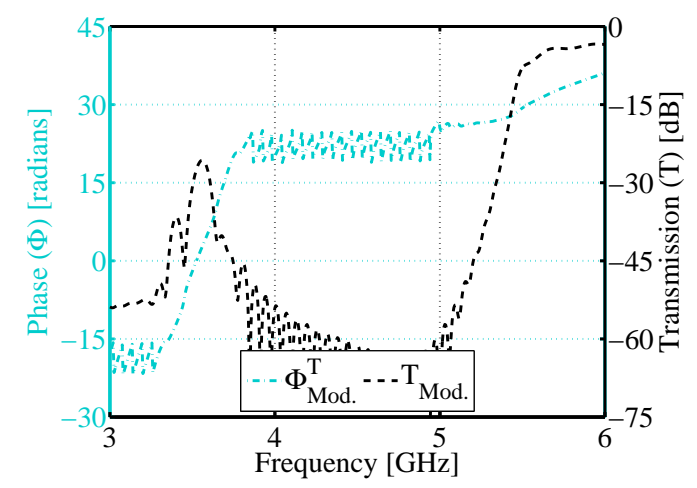

(b) Exp. 2-D HFL Trans. Phase

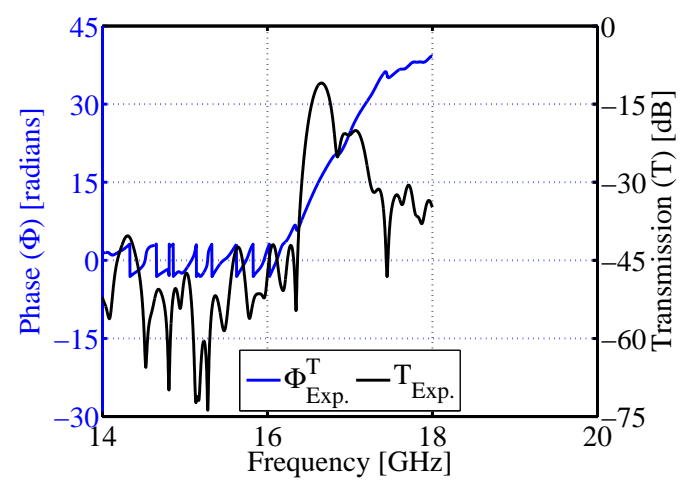

(d) Mod. 2-D HFL Trans. Phase

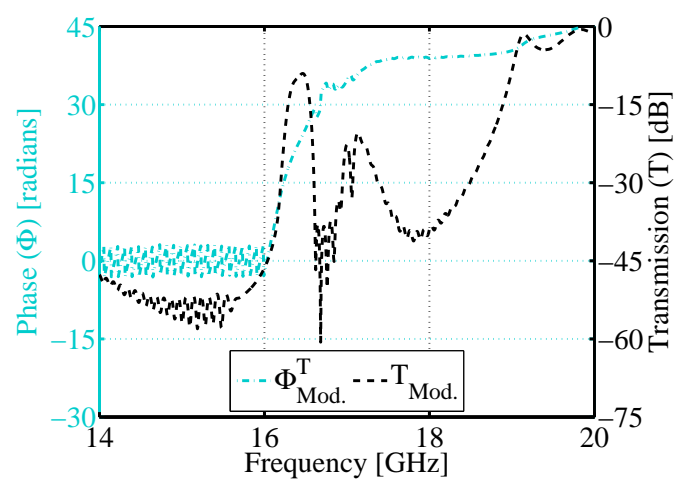

(e) Teflon Reference Trans. Phase

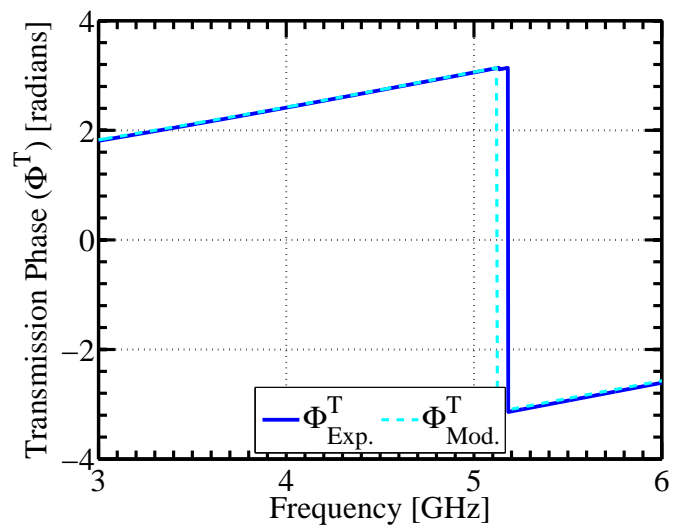

Figure 4.3: Transmitted (T) phase $\Phi$ of the LFL, HFL and Teflon reference sample are presented for experiment (Exp.) and modeling (Mod.). (a-d) The transmitted phase is overlaid with transmission magnitude. The high number of oscillations in the transmitted phase correlated with very low transmission coefficients. (e) transmitted phase of the Teflon reference sample. 
to drastic changes in index of refraction over a narrow frequency band. The transmitted phase away from resonance changed less drastically, indicating a region with ordinary dispersion.

The measurements were in good agreement with computational predictions. Measurement inaccuracies were attributable to sample placement, edge effects, as well as mathematical effects inherent to the Fourier transforms of the time domain gating technique. Differences in frequency response between modeling and experiment were investigated by reevaluating the model with variations of the substrate permittivity, and MTM constituent element feature sizes. The variations were based on the manufacturer's specified tolerances for the dielectric constants of the substrate and the fabrication accuracy of the printed circuit board technique for the rod trace width $\left(\operatorname{Rod}_{h}\right)$, SRR trace width $\left(S R R_{h}\right)$, and SRR gap size $\left(S R R_{d}\right)$, as is shown in fig. 4.4 and fig. 4.5. For both lenses, it was found that fine control over the substrate's dielectric properties altered the resonance response of the MTM. Changes to the real part of permittivity $\left(\epsilon^{\prime}\right)$ shifted the frequency; lower $\epsilon^{\prime}$ resulted in higher resonance frequency, higher $\epsilon^{\prime}$ in lower resonance frequency. Changes to the imaginary part of permittivity $\left(\epsilon^{\prime \prime}\right)$ of the substrate altered the transmission coefficient while maintaining the resonance frequency. The MTM constituent elements were manufactured with a tolerance of $\pm 0.026 \mathrm{~mm}$. Higher SRR gap width, and smaller SRR element width resulted in a resonance shift to higher frequencies, and vice-versa. Changes to the rod width provided negligible effects. The combination of changes in $\epsilon^{\prime}$ and physical dimensions could account for the frequency discrepancy between experiment and modeling. Further contributing factors could be due to the variation in unit cell spacing stemming from improper alignment of the fabricated MTM boards.

The transmission and reflection data were the basis for analyzing the electromagnetic properties of the MTM lenses. Measured and calculated S-parameter data allowed for deriving critical information for the index of refraction of these MTM lenses. 
(a) Substrate permittivity $\epsilon^{\prime}$

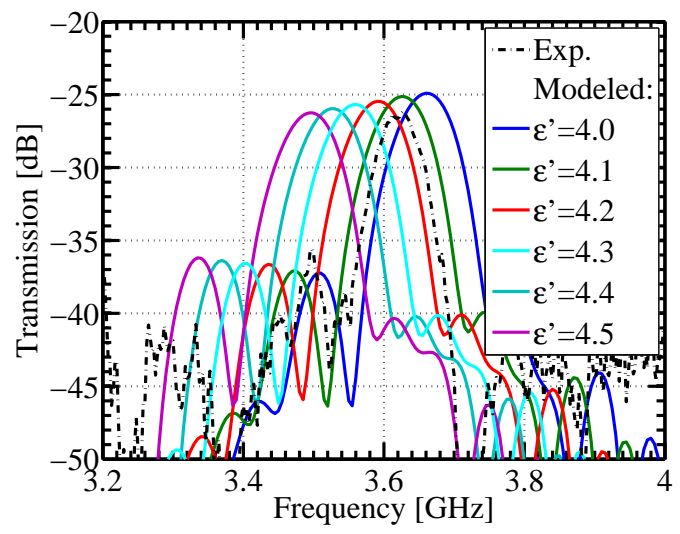

(c) SRR trace width $S R R_{h}$

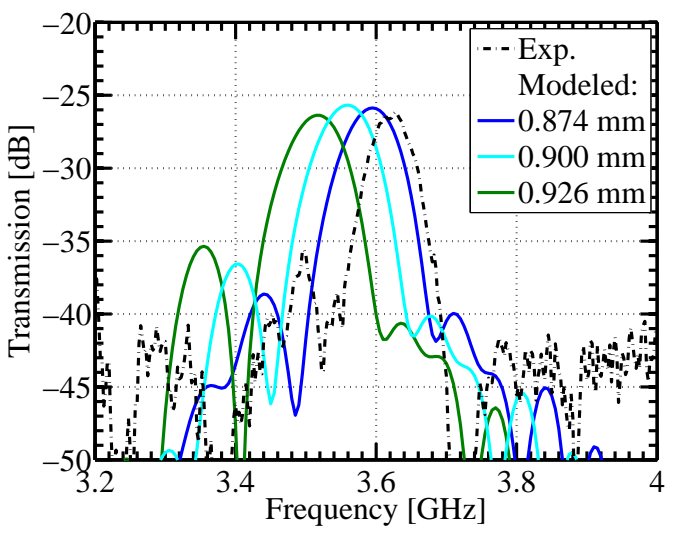

(b) Substrate permittivity $\epsilon^{\prime \prime}$

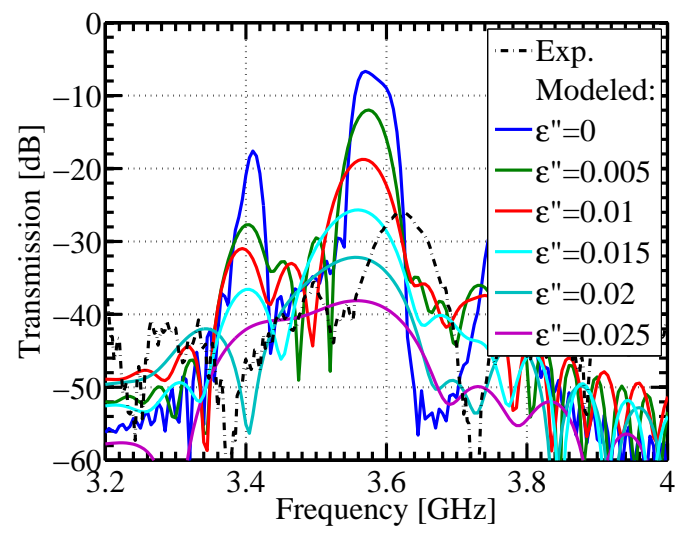

(d) SRR gap size $S R R_{d}$

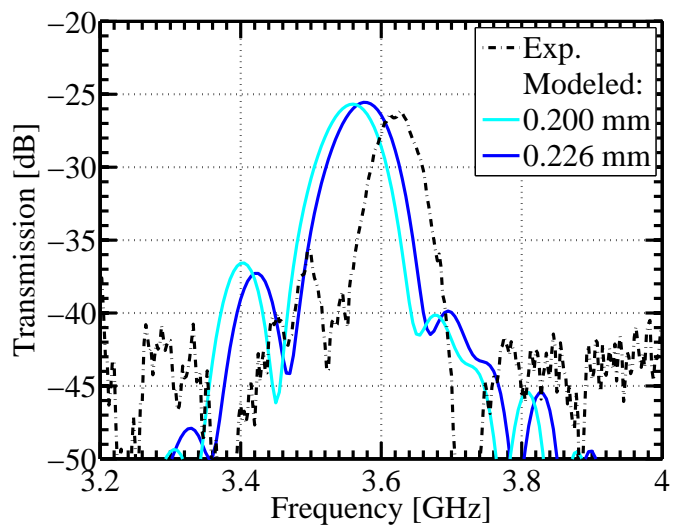

(e) Rod trace width $\operatorname{Rod}_{h}$

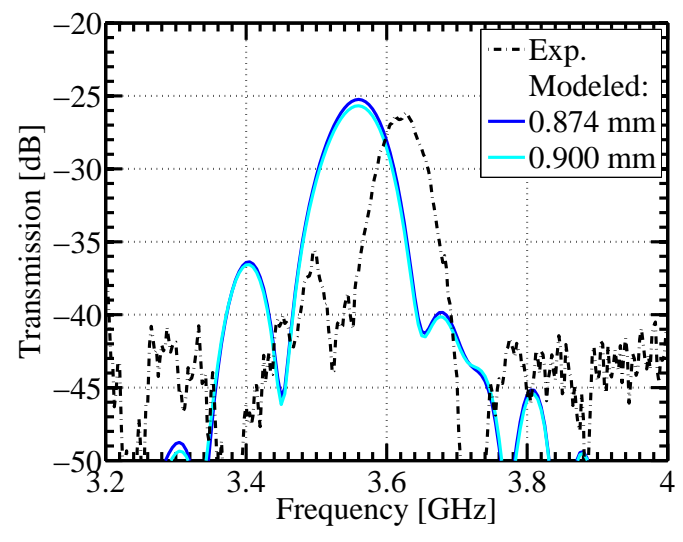

Figure 4.4: Investigation into effect of tolerances onto modeled transmission for 2-D LFL. Transmission was modeled for various MTM constituent element feature sizes and substrate dielectric constant. The modeled results are compared to the experimental data (Exp.). Specified values were from the substrate manufacturer's specifications and desired feature sizes. (a) $\epsilon^{\prime}$ specified value was 4.3 , (b) $\epsilon$ " specified value was 0.015 , (c) $S R R_{h}$ specified width was $0.9 \mathrm{~mm}$, (d) $S R R_{d}$ specified gap was $0.2 \mathrm{~mm}$, and (e) $\operatorname{Rod}_{h}$ specified width was $0.9 \mathrm{~mm}$. 
(a) Substrate permittivity $\epsilon^{\prime}$

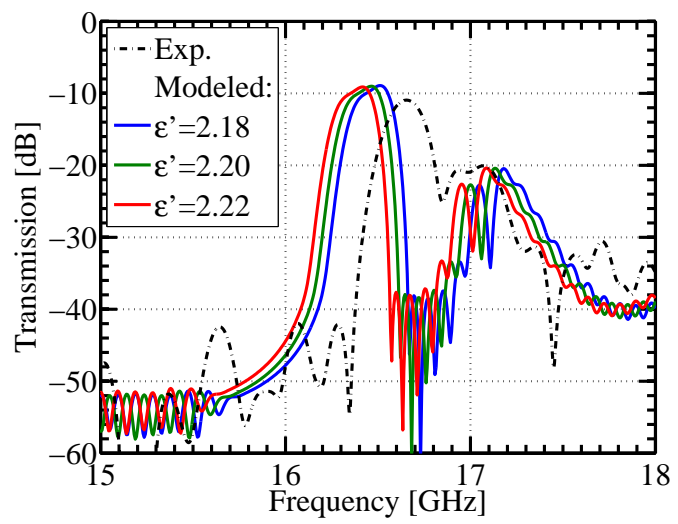

(c) SRR trace width $S R R_{h}$

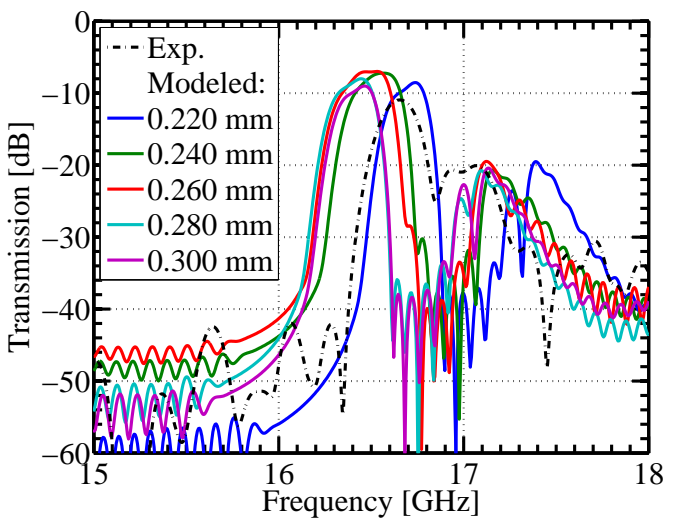

(b) Substrate permittivity $\epsilon^{\prime \prime}$

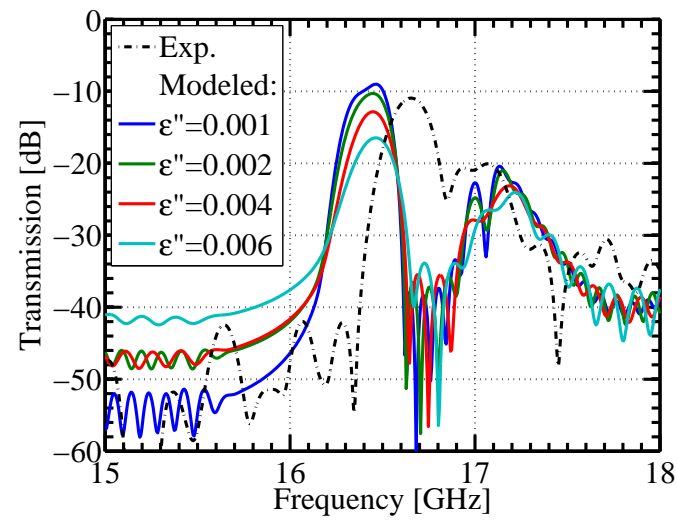

(d) SRR gap size $S R R_{d}$

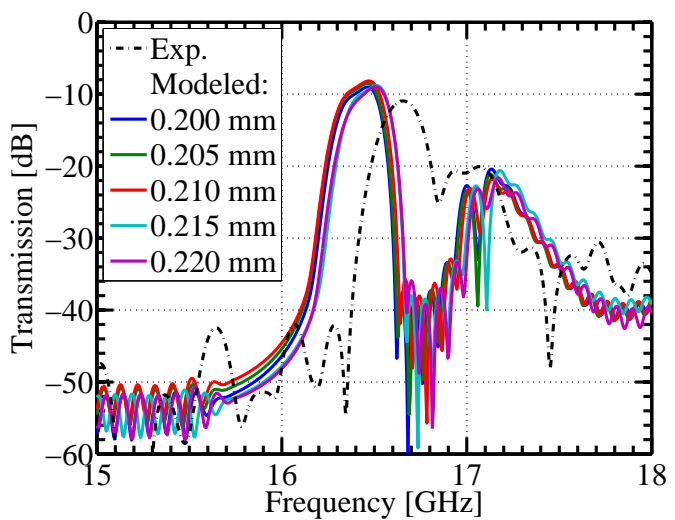

(e) Rod trace width $\operatorname{Rod}_{h}$

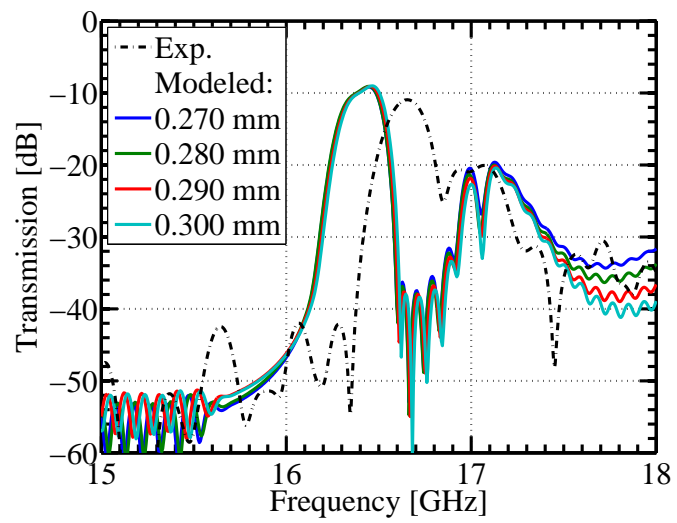

Figure 4.5: Investigation into the effect of tolerances onto modeled transmission for 2-D LFL. Modeled transmission coefficient of the 2-D HFL was evaluated for tolerances in substrate dielectric constant and MTM constituent element feature sizes. The modeled results are compared to the experimental data (Exp.). Specified values were from the substrate manufacturer's specifications and desired feature sizes. (a) $\epsilon^{\prime}$ specified value was 2.2, (b) $\epsilon^{\prime \prime}$ specified value was 0.0009 , (c) $S R R_{h}$ specified width was $0.3 \mathrm{~mm}$, (d) $S R R_{d}$ specified gap was $0.2 \mathrm{~mm}$, and (e) $\operatorname{Rod}_{h}$ specified width was $0.3 \mathrm{~mm}$. 


\subsection{Index of Refraction}

As MTM theory promises superlensing for $n_{\text {eff }}=-1+i 0$, it is advantageous to know the frequency at which this condition is satisfied. The index of refraction was retrieved from the S-parameter data for the 2-D LFL and 2-D HFLs. Literature has outlined several retrieval methodologies. These methods showed that computations were not necessarily straightforward as they were complicated by multiple solutions to the real part of $n_{\text {eff }}\left(\boldsymbol{R e}\left(n_{e f f}\right)\right)$. Chen et al. [47] introduced a robust index of refraction calculation that solved for the correct branch of $\mathbf{R e}\left(n_{\text {eff }}\right)$. The retrieval methodologies found in literature [47-49] have been implemented in MathWorks Matlab@). As the metamaterial lacks mirror symmetries, the overall structure exhibits bianisotropy, therefore the effective impedance $z_{\text {eff }}$ depends on the four S-parameters given as follows [48]:

$$
z_{e f f}=\frac{\left(S_{11}-S_{22}\right) \pm \sqrt{\left(1-S_{11} S_{22}+S_{21} S_{12}\right)^{2}-S_{21} S_{12}}}{\left(1-S_{11}\right)\left(1-S_{22}\right)-S_{21} S_{12}}
$$

The formula for the index of refraction was derived from the plane wave solution at normal incidence onto a slab of material.

$$
\chi=e^{i n_{e f f} k_{0} d}=\frac{S_{21}}{1-S_{11} \frac{z_{e f f}-1}{z_{e f f}+1}}
$$

Where the arbitrary variable $\chi$ depends on $n_{e f f}$, the free-space wavenumber $\left(k_{0}\right)$, and the thickness of the MTM lens $(d)$. Since the MTM is a passive medium, the sign of the root in eq. 4.1 and eq. 4.2 was chosen such that $\operatorname{Re}\left(z_{e f f}\right) \geq 0$ and $|\chi| \leq 1$ [47-49].

$$
n_{e f f}=n^{\prime}+i \kappa=\frac{1}{k_{0} d}[\operatorname{Im}(\ln (\chi))+2 \mathbf{m} \pi]-\frac{i}{k_{0} d}[\operatorname{Re}(\ln (\chi))]
$$

where

$$
n^{\prime}=\frac{1}{k_{0} d}[\operatorname{Im}(\ln (\chi))+2 \mathbf{m} \pi] \quad \text { and } \quad \kappa=\frac{-1}{k_{0} d}[\operatorname{Re}(\ln (\chi))]
$$


Solving eq. 4.2 for $n_{\text {eff }}$ (eq. 4.3) shows that the real part of $n_{e f f}, n^{\prime}$, is complicated by multiple solutions of $2 \mathbf{m} \pi$, where $\mathbf{m}$ is an integer. The correct branch of $\mathbf{m}$ is found by first applying a correction based on the Kramers-Kronig relation [49], which relates the real part of the index of refraction to the complex component $\kappa$.

$$
n^{K K}\left(\omega^{\prime}\right)=1+\frac{2}{\pi} \mathcal{P} \int_{0}^{\infty} \frac{\omega \kappa(\omega)}{\omega^{2}-\omega^{\prime 2}} d \omega
$$

$n^{K K}$ denotes the real part of the index retrieved from the Kramers-Kronig method, and $\mathcal{P}$ is the principal value of the improper integral over all frequencies. For proper evaluation of eq. 4.4 over available frequencies the integral is split into two parts and evaluated numerically, as outlined by Szabo et al. [49].

$$
n^{K K}\left(\omega_{j}\right)=1+\frac{\Delta \omega}{\pi}\left(\sum_{v=1}^{j-2} \Psi_{j, v}+\sum_{v=j+1}^{\mathcal{N}-1} \Psi_{j, v}\right)
$$

and

$$
\Psi_{j, v}=\frac{\omega_{v} \kappa\left(\omega_{v}\right)}{\omega_{v}^{2}-\omega_{j}^{2}}+\frac{\omega_{v+1} \kappa\left(\omega_{v+1}\right)}{\omega_{v+1}^{2}-\omega_{j}^{2}}
$$

The solution to Kramers-Kronig was evaluated using eq. 4.5 for all available frequencies, where $\Delta \omega$ denotes the frequency step size and $\mathcal{N}$ the number of frequency steps. Subsequently, $n^{K K}$ can be used to derive the correct branch of $\mathbf{m}$ by setting $n^{K K}$ equal to $n^{\prime}$ from eq. 4.3.

$$
\mathbf{m}=\operatorname{rnd}\left[\left(n^{K K}-\frac{1}{k_{0} d} \operatorname{Im}[\ln (\chi)]\right) \frac{k_{0} d}{2 \pi}\right]
$$

$\mathbf{m}$ is defined by rounding the difference between $n^{K K}$ and the initial solution of $n^{\prime}$ to the nearest integer.

Unfortunately, the extraction of $\mathbf{m}$ is susceptible to rapid oscillations in phase of the S-parameter data. The multiple phase wrapping in S-parameter data results in unphysical oscillations in the extracted $n_{\text {eff }}[47,49]$. In experiment, phase oscillations can be expected from measurements near the noise floor $S_{21}$ and $S_{12} \leq-40 \mathrm{~dB}$. In modeled data the 
phase errors are due to limitations in computational resources for high discrepancy between wavelength and SRR and RE feature sizes. More refined modeling efforts can resolve some of the phase errors; however, this requires very long computational times. Therefore, index of refraction retrieval is not performed at frequency regions where the transmission is close to the noise floor of the experiment or very high oscillations in transmitted phase are present. For modeling, similar limitations are chosen. The correct branch of $\mathbf{m}$ is chosen by first unwrapping the phase of the transmitted microwave over valid frequency ranges. Furthermore, it is known that superlensing can only occur for $n_{\text {eff }} \approx-1$, therefore $\mathbf{m}$ was chosen such that the resonance frequency of the MTM exhibits $n^{\prime} \approx-1$. The $n_{\text {eff }}$ was calculated from modeling and experiment, and results are presented in fig. 4.6.

Experimental and modeled results follow each other well. For the lenses, discrepancies between modeling and experiment were due to multiple phase wrapping found in modeling, compared to lower number of phase wrappings found in the experiment. Slight mismatches in frequency are results of differences in S-parameter data. For the Teflon reference sample the retrieval method works well, as the index of refraction is nearly identical. Retrieval methodology breaks down slightly in the region where the effective thickness of the sample is close to the quarter-wavelength of the microwave radiation.

The frequency for which $n^{\prime}=-1$ did not necessarily coincide with the measured resonance frequency. The table 4.1 gives frequency, retrieved $n^{\prime}, \kappa$, and transmission values for the MTMs resonance, $n_{e f f}=-1$, and at the frequencies chosen for imaging. For the purposes of modeling the imaging properties of the MTM lenses, the table shows the frequency used and the material parameters of the dummy material.

Theory predicted ideal imaging performance at the $n_{e f f}=-1+i 0$ case. However, it is important to point out that imaging resolution is tied to the enhancement of evanescent waves. The absorption of the MTM lenses, which is tied to the imaginary part of the index of refraction $\kappa$, governs this enhancement. In order to achieve the best imaging performance, trade-offs were made between the ideal index of refraction and the extinction coefficient. The 
extinction coefficient dominates the resolution performance of the MTM lenses and has to be minimized, whereas a shift in index of refraction shifts the focus of the lens. Therefore, imaging frequency for the MTM lenses was chosen to be $3.658 \mathrm{GHz}$ for the 2-D LFL and

(a) 2-D Low Frequency Lens

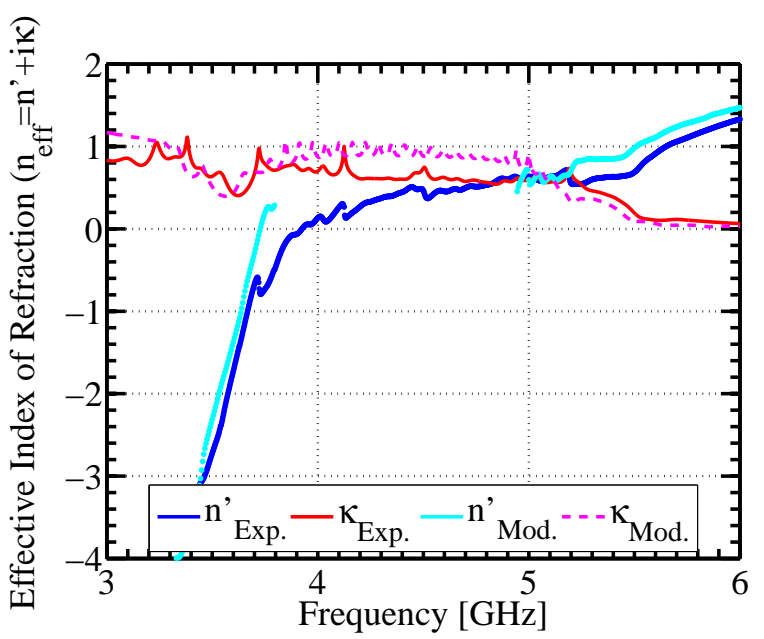

(b) 2-D High Frequency Lens

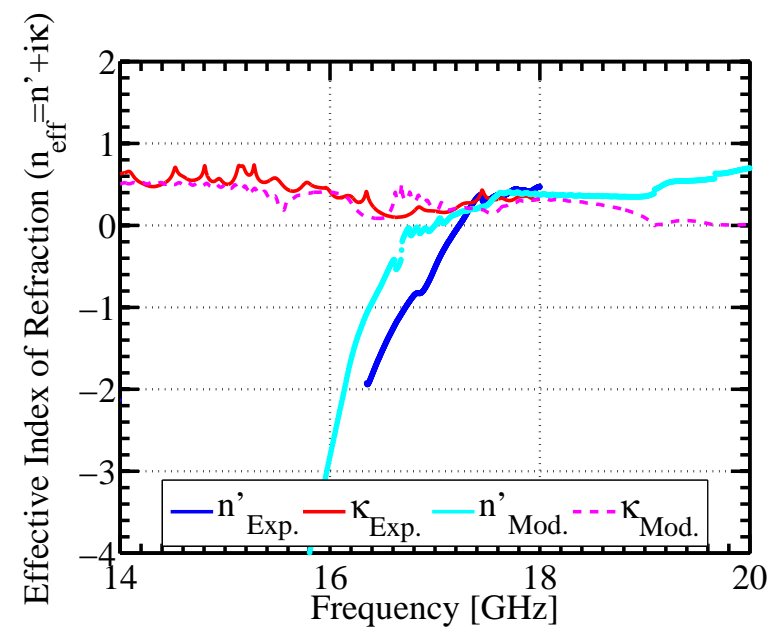

(c) Teflon Reference Sample

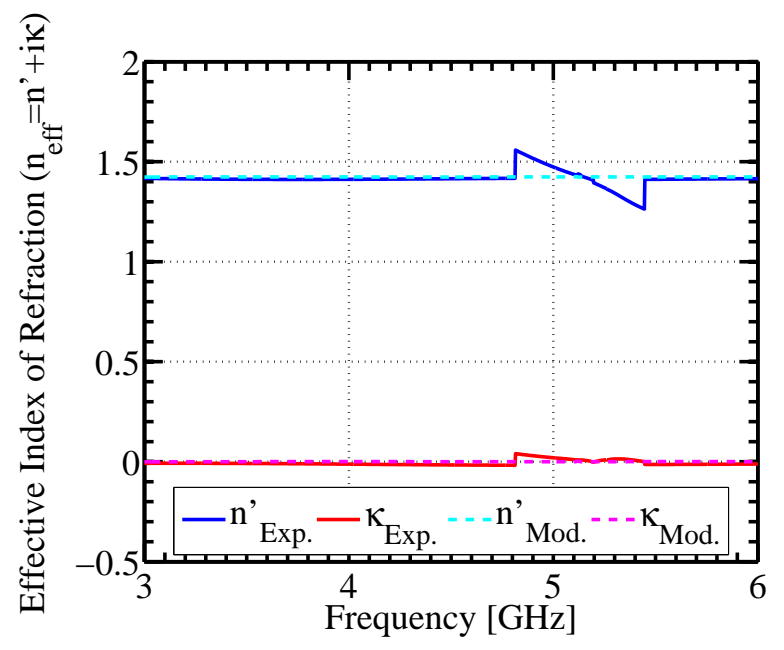

Figure 4.6: Retrieved $n_{\text {eff }}$ for 2-D LFL, 2-D HFL, and Teflon reference. The real $n^{\prime}$ and imaginary $\kappa$ parts of the index of refraction are compared between Experimental data (Exp.) and modeled results (Mod.). Experiment and modeled results are in good agreement with each other. Modeled data in (a) exhibits a region without solutions to the retrieval methodology due to large oscillations in phase. Experiment in (b) was cut off due to data near the noise floor. Experimental results of the Teflon reference sample is in very good agreement with modeling, albeit showing discrepancies due to phase errors where the thickness of the sample was close to $1 / 4$ of the wavelength of the MW. (b) reproduced with permission of author [71]. 
Table 4.1: Retrieved indices of refraction of the MTM lenses. Values are given for the resonance frequency, $n_{e f f}=-1$, and the frequency for which imaging was chosen. The resonance frequency is defined at the peak transmission of the resonance band. The superlensing requirement stipulates that $n^{\prime}=-1$. The imaging frequency was chosen in a trade-off between $n^{\prime} \approx-1$ and the extinction coefficient $\kappa$ at its minimum.

\begin{tabular}{|c|c|c|c|c|c|}
\hline & & \multicolumn{2}{|c|}{ 2-D LFL } & \multicolumn{2}{|c|}{ 2-D HFL } \\
\hline & & Experiment & Modeling & Experiment & Modeling \\
\hline 8 & Freq (GHz) & 3.620 & 3.560 & 16.654 & 16.466 \\
\hline สี & Trans. (dB) & -26.06 & -25.68 & -10.94 & -9.01 \\
\hline 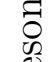 & $n^{\prime}$ & -1.51 & -1.74 & -1.14 & -0.78 \\
\hline 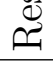 & $\kappa$ & 0.40 & 0.39 & 0.10 & 0.09 \\
\hline 7 & Freq $(\mathrm{GHz})$ & 3.668 & 3.635 & 16.722 & 16.376 \\
\hline 1 & Trans. (dB) & -31.04 & -37.59 & -12.30 & -10.28 \\
\hline & $n^{\prime}$ & -1.00 & -1.02 & -0.99 & -1.00 \\
\hline$\approx$ & $\kappa$ & 0.49 & 0.61 & 0.12 & 0.10 \\
\hline & 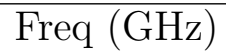 & 3.658 & 3.620 & 16.672 & 16.672 \\
\hline$\Xi$ & Trans. (dB) & -29.25 & $\mathrm{~N} / \mathrm{A}$ & -11.03 & $\mathrm{~N} / \mathrm{A}$ \\
\hline$\widetilde{\sigma}$ & $n^{\prime}$ & -1.11 & -1.01 & -1.10 & -1.00 \\
\hline$\exists$ & $\kappa$ & 0.46 & $8.8 \times 10^{-3}$ & 0.10 & $1.9 \times 10^{-6}$ \\
\hline
\end{tabular}

16.672 GHz for the 2-D HFL. These frequencies dictated the design of the receive antennas for the detectors and the source antennas in the imaging experiments. Furthermore, modeling of the lenses' imaging characteristics were evaluated at these frequencies. 


\subsection{Polarization Rotation by the Metamaterial Lens}

In the measurement, where the source and receive horn were held in parallel polarization with respect to each other, the quantitative predictions were similar to the S-parameter measurements. Computations were performed for incident plane waves polarized along the $\mathrm{x}$ - $\left(0^{\circ}\right)$ and $\mathrm{y}$-axis $\left(90^{\circ}\right)$; whereas the experiment varied the incident polarization angle with respect to the lens, which is aligned along the y-axis $\left(90^{\circ}\right)$. Transmission was analyzed for its $\vec{E}$ components along the principal axes. The MTM resonant regions and start of the passband were evaluated for frequencies of $3 \mathrm{GHz}$ to $6 \mathrm{GHz}$. In the ordinary incident MW polarization condition $\left(90^{\circ}\right)$, the 2-D LFL (see fig. 4.7a)and 1-D LFL (see fig. 4.7b) exhibited the characteristic transmission profile of a NIM, as both SRR and RE constituent elements were excited by the incident MW. The Crossboard LFL (see fig. 4.7c), did not exhibit a resonance, as the incident polarization did not allow for the SRRs to contribute towards a negative $\mu_{\text {eff }}$. With the MWs incident polarization along $0^{\circ}$, the $\vec{E}$ of the MW is perpendicular to the REs and thus the REs were not excited. Furthermore, the incident MWs were only able to excite SRRs that were perpendicular to the propagation direction of incident MWs. Therefore, at resonance, the 2-D LFL, and Crossboard LFL exhibited negative $\mu_{e f f}$, whereas the 1-D LFL behaved as an ordinary material with positive $n_{\text {eff }}$.

In the second experiment, the receive horn was rotated to analyze the polarization state of the transmitted MW. The resonance frequency of $3.63 \mathrm{GHz}$ was chosen as it represents the mean resonant frequency of the 1-D and 2-D MTM resonances. It was found that, at a resonance frequency of $3.63 \mathrm{GHz}$, the transmission response of SRR and RE MTM structures was highly sensitive to the polarization state of the incident microwave. This sensitivity was due to the coupling mechanisms between the incident radiation and the MTM lens' constituent elements [76]. For incident polarization along $\vec{E}_{90^{\circ}}$ (see fig. 4.8a), the 2-D LFL altered the polarization state of the incident radiation into elliptical polarization with a tilt along the principal axis, where the ellipticity changed with incident polarization angle. The 1-D LFL did not exhibit a change in polarization state. At incident polarization of $\vec{E}_{45^{\circ}}$ 
(a) 2-D LFL

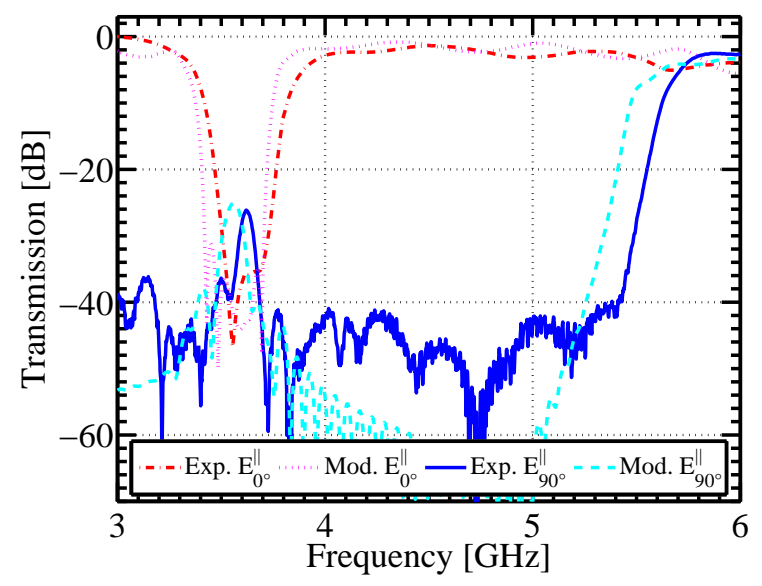

(b) 1-D LFL

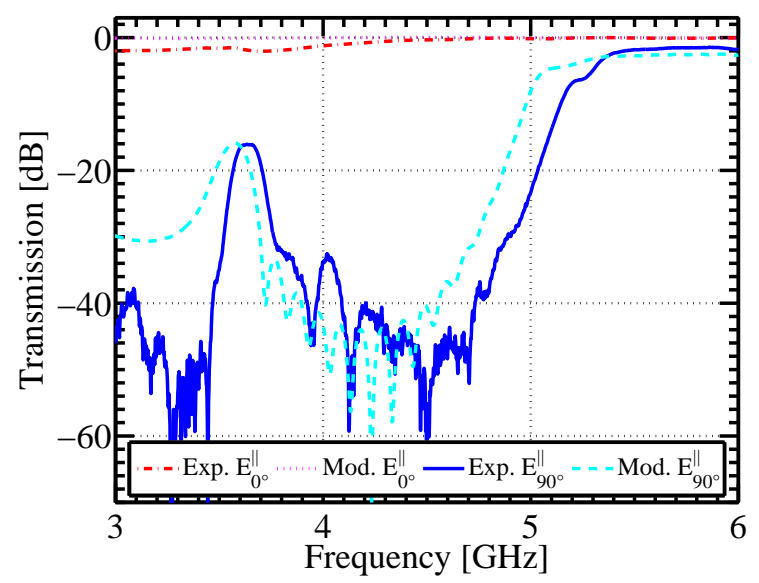

(c) Crossboard LFL

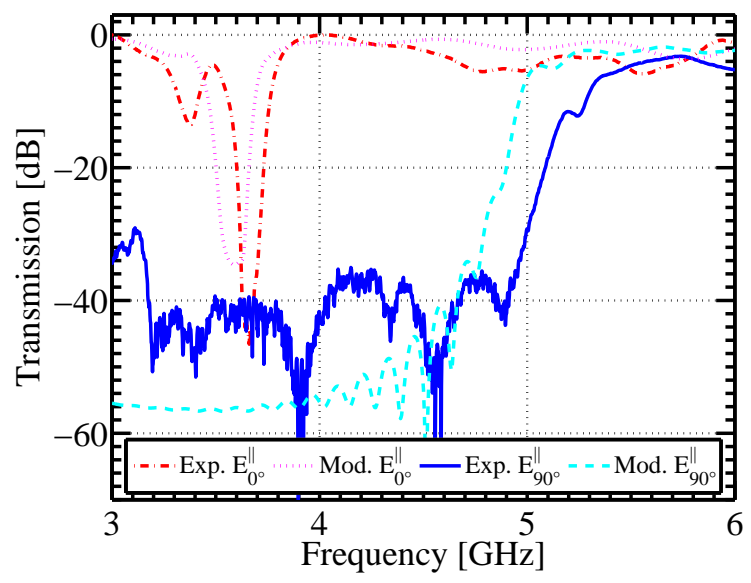

Figure 4.7: Incident polarization effect on transmission of MTM lens configurations: 2-D LFL (a), 1-D LFL (b), and Crossboard (c) configuration. Transmission in voltage (dB) vs. frequency $(\mathrm{GHz})$ for $\vec{E}_{0^{\circ}}^{\|}$and $\vec{E}_{90^{\circ}}^{\|}$incident polarization with receive and transmit horns in parallel for the different lens configurations. The experimental data is in good agreement with computational predictions. The resonance frequency is $3.55 \mathrm{GHz}$ for modeling and $3.63 \mathrm{GHz}$ for experiment. Figures are taken from $[72,73]$.

(see fig. 4.8b), the polarization state of the transmitted MW is determined by the vector components of the polarization state and hence contributions from $\vec{E}_{90^{\circ}}$ and $\vec{E}_{0^{\circ}}$ incident MWs. For the 2-D LFL, the contribution from $\vec{E}_{90^{\circ}}$ incident MW dominates the polarization state, whereas for the 1-D LFL, the $\vec{E}_{0^{\circ}}$ contribution dominates. For incident polarization along $\vec{E}_{0^{\circ}}$ (see fig. $4.8 \mathrm{c}$ ) the polarization state of the transmitted MW was unaffected by the 2-D and 1-D MTM LFL. The 1-D lens did not affect the polarization of the incident 
microwave radiation. However, drastic changes in the transmission profile and $n_{\text {eff }}$ were found. These changes were due to the incident polarization state $\left(\vec{E}_{90^{\circ}}\right.$ and $\left.\vec{E}_{0^{\circ}}\right)$ coupling with the metamaterial lens constituent elements.

(a) Incident Polarization Along $90^{\circ}$ (Y-axis)

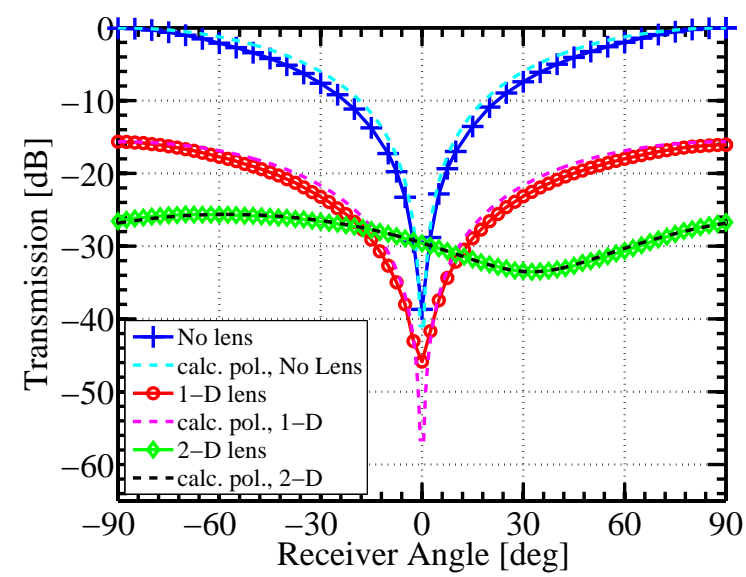

(b) Inicident Polarization Along $45^{\circ}$

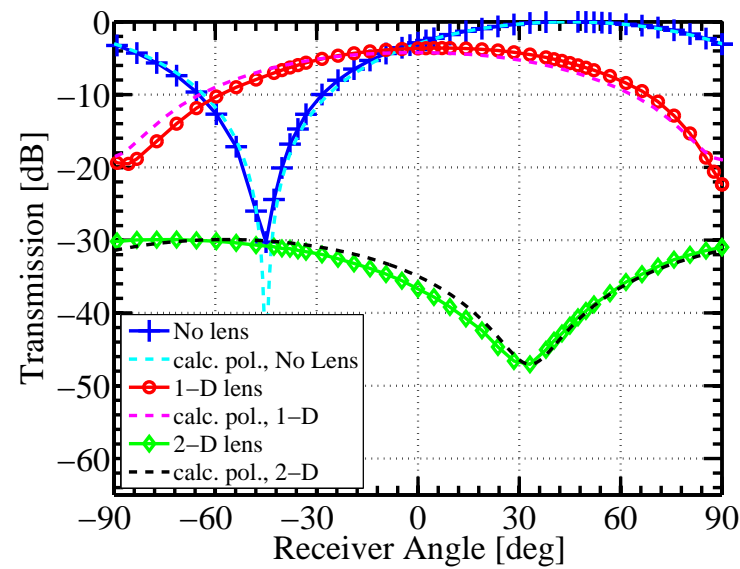

(c) Incident Polarization Along $0^{\circ}$ (X-axis)

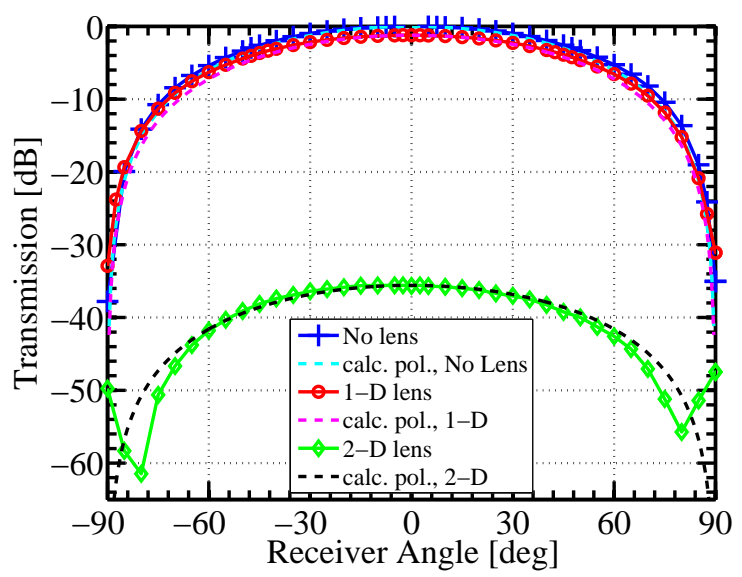

Figure 4.8: Polarization rotation for 2-D LFL, 1-D LFL, and No-Lens. Transmission in voltage (dB) vs. receive horn polarization angle in degrees for various source angles (a) $90^{\circ}$, (b) $45^{\circ}$, and (c) $0^{\circ}$. Measurements for the 2-D LFL, 1-D LFL and "No Lens" are compared to calculated polarization values. Figures are taken from $[72,73]$.

The control of polarization state of the incident MW radiation allows tailoring of the transmission, which can be leveraged towards novel transmission based MW MTM sensors. The overall effect was not always easily interpretable from physical reasoning due to the magneto-electric coupling between SRRs of different orientations. More importantly, this 
study presented the magnitude of change and effect of the polarization properties in fabricated SRR and RE systems. The transmitted MW radiation was significantly affected by the MTM resonant elements and had to be taken into careful consideration in the design of practical SRR and RE based systems. As MTM components such as lenses are deployed in sensor and NDE applications, consideration of the polarization properties of these devices will open new opportunities for the development of novel sensors and devices. 


\section{Chapter 5: Imaging Study Results}

\section{and Discussion}

Imaging was separated into two configurations. Transmission mode imaging, which imaged back illuminated objects, served as a test bed for the imaging properties of the MTM lenses. Reflection mode imaging which co-located the imaging system to one side, presented the most practical system in a typical application.

\subsection{Transmission Mode Imaging}

\subsubsection{Low Frequency Lens}

The 2-D LFL was investigated for its focusing properties and the 1-D LFL for its imaging capabilities. Results from the 2-D LFL experiment verified the imaging setup with the use of a single dipole antenna source instead of a back illuminated aperture. In this simplified configuration, the focus spot of the 2-D LFL dictated the resolution of the lens. The resolution was defined as the full width at half maximum (FWHM) of the received power at focus of the lens. As MTM flat-lenses exhibit imaging conditions dependent on the object to lens distance $f_{1}$ and thickness of the lens as described in equation 2.12, measurements were taken at four standoff distances (17 mm, $24 \mathrm{~mm}, 47 \mathrm{~mm}$, and $70 \mathrm{~mm}$ ).

Equation 2.12 predicted the focal lengths $f_{2}$ to be $66.8,59.8,36.8$, and $13.8 \mathrm{~mm}$ for the respective stand off distances, where as the experimental results in fig. $5.1 \mathrm{~b}$ showed $f_{2}=$ 66, 61, 36 and $16 \mathrm{~mm}$, respectively. The experiment followed the quantitative predictions well. However, the lens did not image below the diffraction limit. This was expected as the 2-D LFL's large extinction coefficient $(\kappa=0.46$ see table 4.1$)$ would not be able to support 
evanescent wave enhancement. Nevertheless, the measurements indicated subwavelength resolution imaging capabilities.

(a) Focal spot distribution for various $f_{1}$

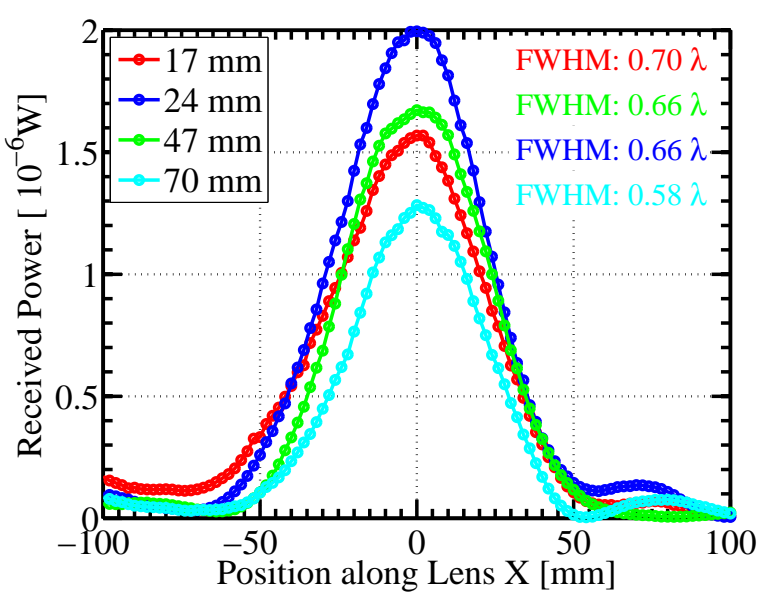

(b) FWHM at various $f_{1}$

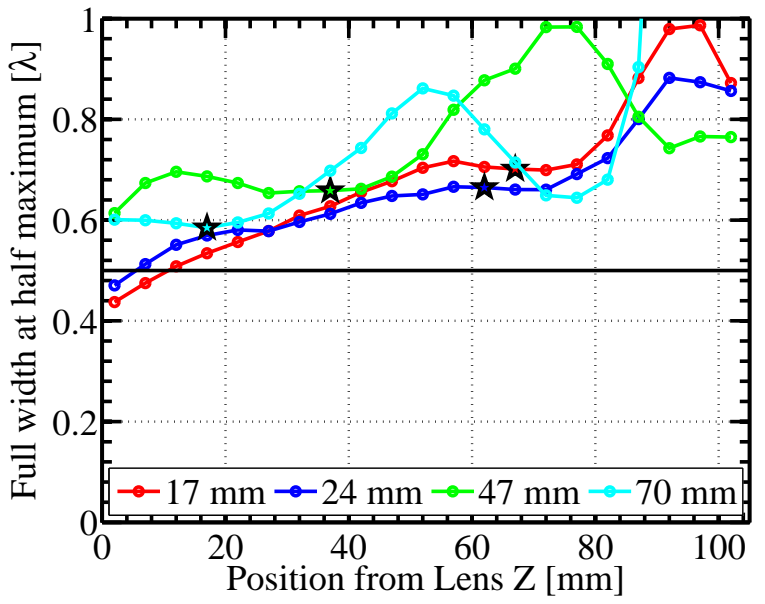

(c) Experimental result of the image plane with $f_{1}=24 \mathrm{~mm}$

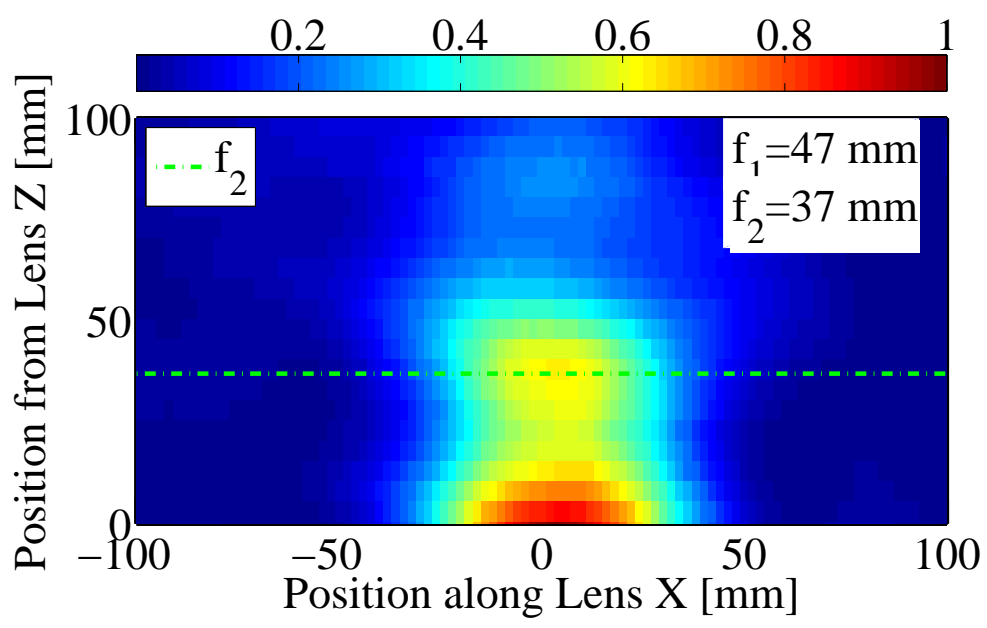

Figure 5.1: 2-D LFL transmission mode imaging characteristics using a dipole antenna source. (a) presents focal spot distributions for various source antenna to lens stand-off distance $\left(f_{1}\right)$. The FWHM declines as $f_{1}$ increases. (b) shows the distribution of the FWHM in the image plane. The focal spot position $f_{2}$ are indicated by $\star$. The focal spot is in the plateaus of the FWHM. FWHM increases due to decreasing near-field information. The diffraction limit $\left(\Delta_{A b b e}=\lambda / 2\right)$ is indicated by the black line. (c) shows the normalized power distribution in the image plane for the dipole at $f_{1}=47 \mathrm{~mm}$ and indicates the focal spot length $f_{2}=37 \mathrm{~mm}$.

In investigating imaging of apertures it was found that the 2-D LFL's images were not very well defined. This was due to several factors. First, the limited physical size of the lens introduced edge effects. Secondly, the SRRs perpendicular to the propagation direction were 
found to have kinked the image, depending on the polarization state of the incident MW. Thirdly, low coefficient of transmission through the 2-D LFL suppressed the dynamic range of the measurement due to elevated levels of errant noise sources.

Subwavelength resolution imaging was achieved for the 1-D LFL with single apertures. The focus spot was well defined and the lens was able to image the subwavelength sized features of the $\lambda / 2$ sized aperture sample, as is shown in fig. 5.2. In the case of the image plane, fig. 5.2a shows that the experimental data was less clear as compared to modeled data, shown in fig. 5.2b. This was thought to be due to a couple of factors. Firstly, the limited size of the 1-D LFL in width and height introduced edge effects, thus deteriorating the image. Secondly, the 1-D LFL did not have cross-boards in its configuration, making imaging of MWs with high angles of incidence difficult. As such, the 1-D LFL was still able to image the subwavelength sized aperture, albeit with a penalty in resolution due to its size, configuration, and constraints from $\kappa$. Compared to modeling, the experiment exhibited a broader FWHM of the imaged aperture. This was due to the fact that modeling used an idealized MTM lens and predicted the electric field in its entirety, as compared to retrieving an image with a dipole antenna.

Comparing the 1-D LFL (fig. 5.2c) to a near-field measurement without the lens (fig. 5.2d), the MTM lens exhibited a small penalty in resolution while significantly increasing the stand-off distance. The 1-D LFL had a resolution of $0.54 \lambda$ and object to image distance of $181 \mathrm{~mm}(2.2 \lambda)$. The near-field image exhibited a resolution of $0.49 \lambda$ with a $4 \mathrm{~mm}(0.05 \lambda)$ stand-off distance. 2-D images from 1-D LFL and near-field measurements of the $\lambda / 2$ aperture are shown in fig. 5.2e and fig. 5.2f. The image in fig. 5.2e shows the limitations of the 1-D LFL. The near-field image in fig. $5.2 \mathrm{f}$ presents a much cleaner picture of the single aperture. Due to the inherent limitations of the LFL, more complex objects such as dual apertures were not able to be imaged. Moreover, modeling of the 2-D and 1-D LFLs revealed that the image quality would be influenced by small lens dimensions, relative to $\lambda$ of the incoming MW. While subwavelength features on the order of $\lambda / 4$ were resolved in the model, edge 
effects from the lens prevented well defined images. The HFL, which was much larger in terms of $\lambda$, proved to be a much better candidate for imaging. Therefore, the LFLs were not used for the reflection mode imaging study. 
(a) 1-D LFL image from experiment, $\mathrm{X}-\mathrm{Z}$ plane

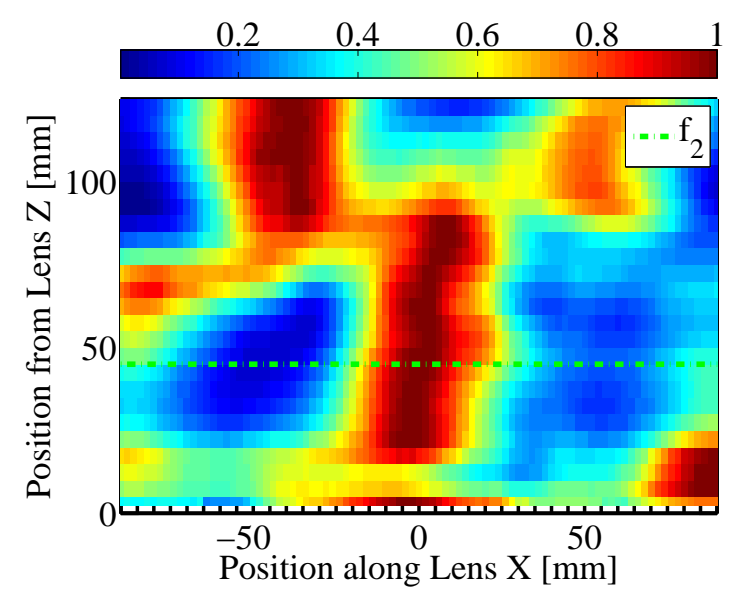

(c) 1-D LFL scan at the focus

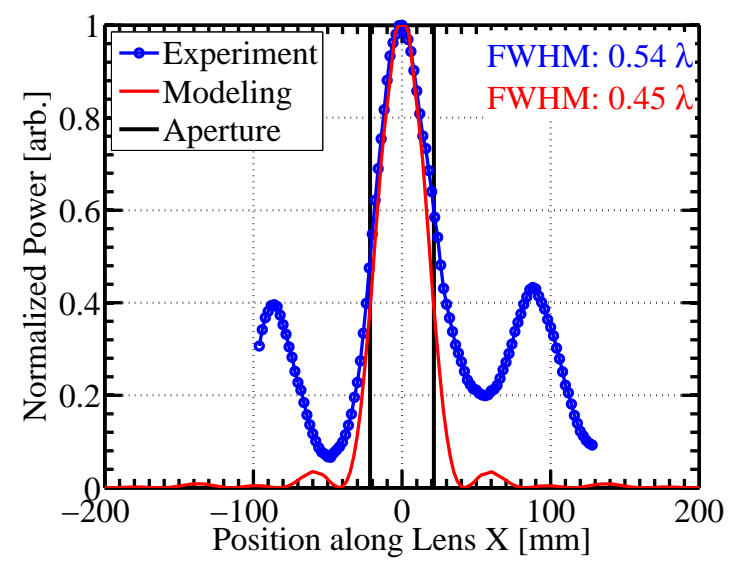

(e) Aperture image with 1-D LFL, X-Y plane

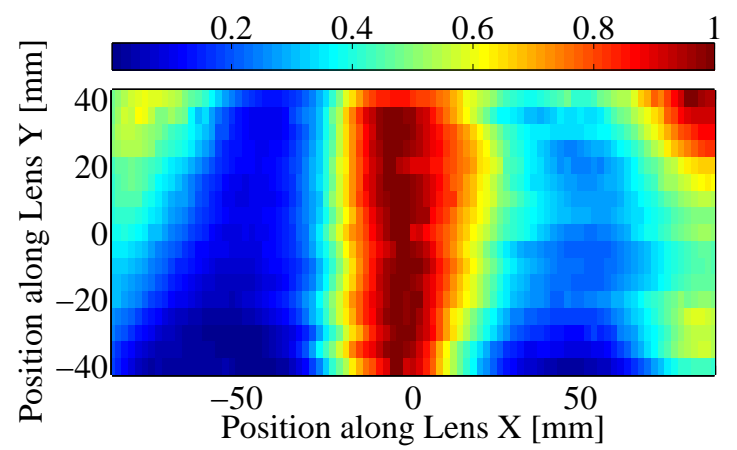

(b) MTM imaging from modeling, X-Z plane

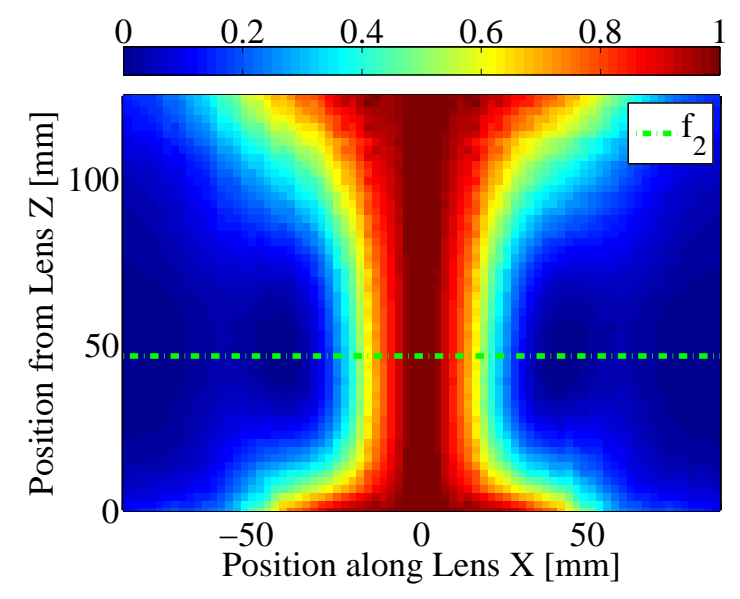

(d) Near-field scan of the aperture

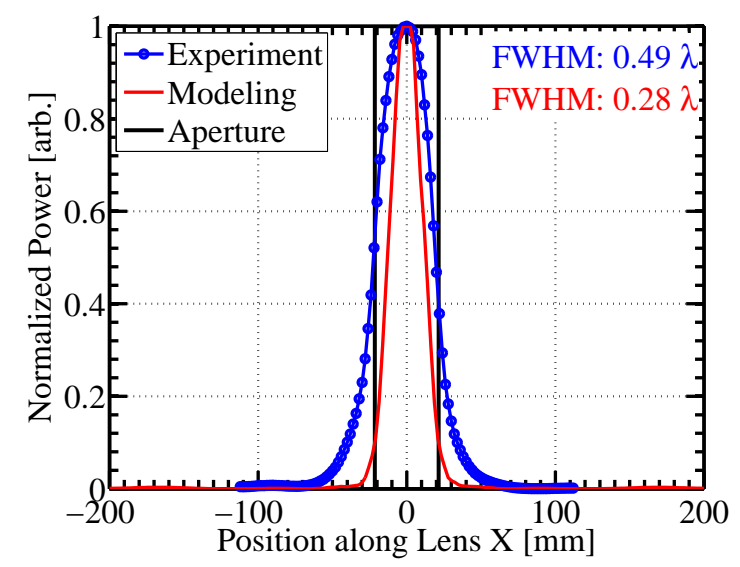

(f) Aperture near-field image, X-Y plane

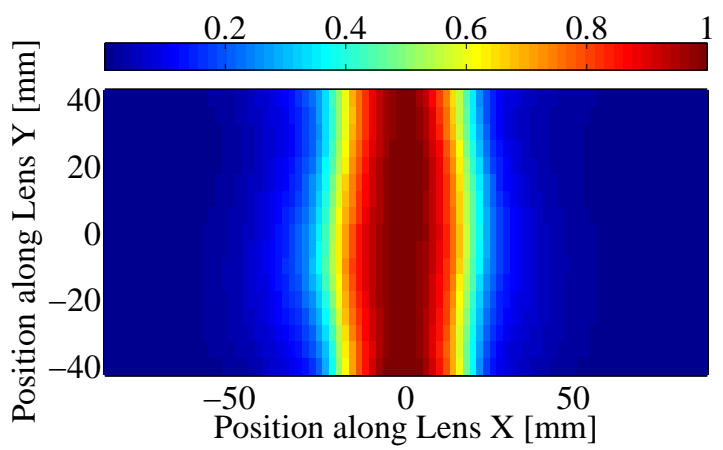

Figure 5.2: 1-D LFL transmission mode imaging characteristics using a single $\lambda / 2$ aperture. (a) and (b) show the X-Z plane, top down view onto the image plane for experiment and modeling using the 1-D LFL. (c) compares aperture image obtained from experiment to modeled data. The object to image distance is $181 \mathrm{~mm}(2.2 \lambda)$. It is of note that the secondary peaks are much larger in the experiment than in modeling. (d) is the near-field measurement and modeling of the aperture. Near-field measurement was taken at $4 \mathrm{~mm}(0.05 \lambda)$ stand-off from the aperture. (e) and (f) show the image of the aperture in the X-Y plane at the focal spot and in the near-field next to the aperture. 


\subsubsection{High Frequency Lens}

The HFL was designed to perform much better than its low frequency counterpart. The lens incorporated low loss substrates, much larger in size (20 $\lambda$ wide, $12 \lambda$ tall and $2 \lambda$ thick), lower extinction coefficient $\kappa=0.10$, and higher frequency. Due to these inherent advantages, the high frequency lens was capable of better resolving aperture samples. The following data are presented and compared to modeling and near-field images: single aperture ( $\lambda$ feature size) data with various stand-off distances (see fig. 5.3 and fig. 5.4), dual apertures with $\lambda / 2$ feature size and $0.75 \lambda$ (fig. 5.5), as well as $\lambda$ separation (fig. 5.6). The imaging frequency for these samples was $16.60 \mathrm{GHz}$ with $n_{\text {eff }}=-1.26+i 0.10$.

Ideal MTM lenses would exhibit a constant FWHM for the permissible stand-off distances, as is shown in fig. 5.3b. Here the FWHM remained constant with some fluctuation due to computational resolution in modeling. In the idealized structure the best focus is determined by the lowest FWHM, as is shown in fig. 5.3d. Here it is clear that the lens comes to a focus with a depth of focus (DOF) of about $15 \pm 1 \mathrm{~mm}$. The DOF is given as the region with full width at half maxima no larger than $\sqrt{2}$ times the smallest FWHM [60].

Like the 2-D LFL, the 2-D HFL exhibits smaller FWHM with increasing $f_{1}$. The effect is thought to be due to geometric advantages from increasing $f_{1}$ as well as non-ideal lensing conditions from $\kappa=0.10$ [67]. As the evanescent wave is weakly restored inside MTM lenses with $\kappa>0$, fine structure information will be greatest at the interface between the lens and image. Therefore it is advantageous to increase the stand-off distance $f_{1}$ such that the focus of the lens coincides with the lens interface, and the maximum amount of recovered evanescent waves can be imaged.

However, there are limitations, as is shown in fig. 5.3c the closest focal distance to the lens does not show the best FWHM. Furthermore, using eq. 2.12, the effective object to image distance for plane wave incidence would be $68 \mathrm{~mm}$; however, angular contributions due to scattering effects or misalignments may change the effective object to image distance. Fig. 5.3c shows a combined object to image distance of $80 \mathrm{~mm}, 78 \mathrm{~mm}$, and $82 \mathrm{~mm}$ for 
(a) Image of single aperture $\lambda$

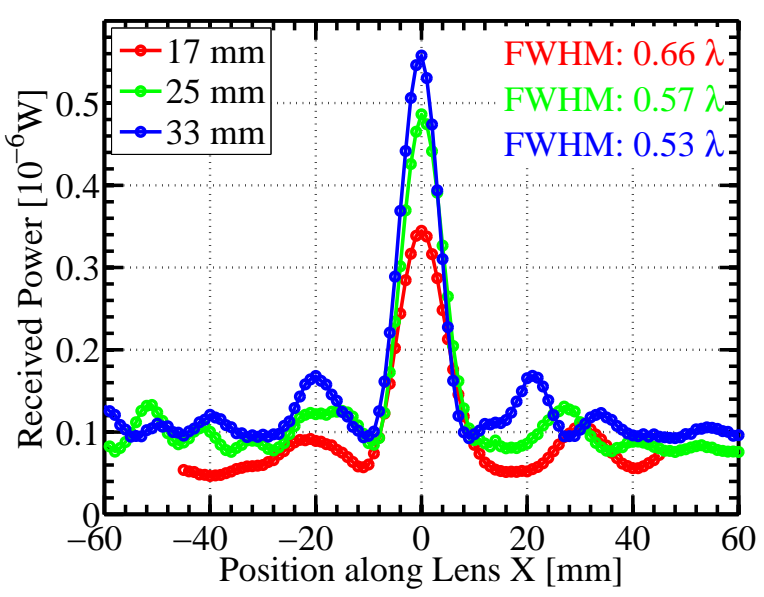

(c) Exp. FWHM for various $f_{1}$

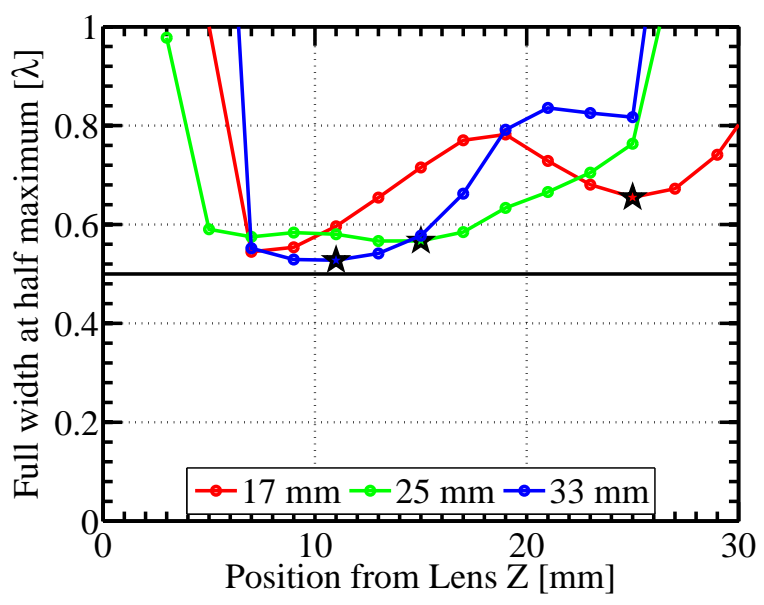

(b) Modeling of single aperture $\lambda$

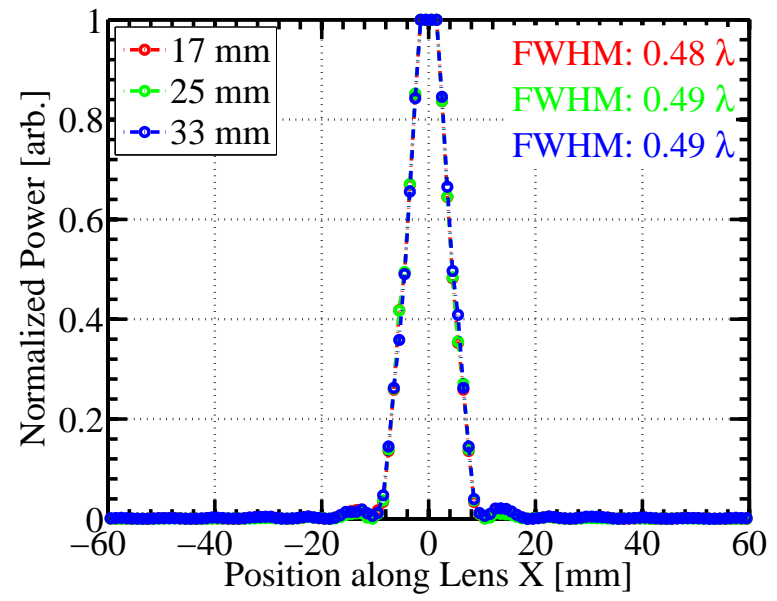

(d) Mod. FWHM for various $f_{1}$

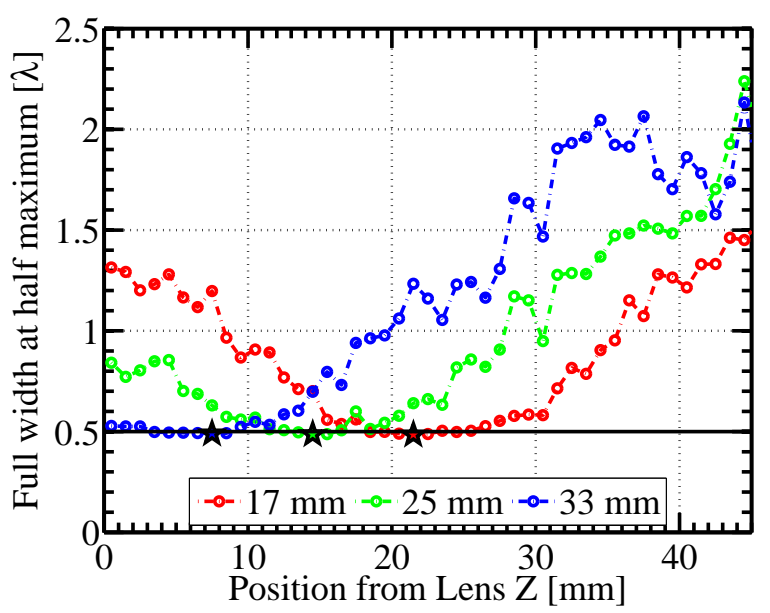

Figure 5.3: HFL transmission mode imaging characteristics for various $\lambda$ sized aperture stand-off distances. Comparison of experiment (a) to modeling (b) of the transmission mode image of a single $\lambda$ sized aperture for various stand-off distances $f_{1}$. The corresponding focus spot in the image plane is denoted by $\star$ in $(c)$ for the experiment and $(d)$ in modeling.

$f_{1}$ equaling $17 \mathrm{~mm}, 25 \mathrm{~mm}$, and $33 \mathrm{~mm}$ respectively, as compared to a predicted $68 \mathrm{~mm}$. The discrepancy is due to angular and geometrical contributions. Angular contributions could result from the interaction of the MW source and the object aperture, and geometrical contributions could result in focusing of propagating waves and recovery of evanescent waves at different $f_{2}$ positions. This would manifest as fluctuations in the FWHM. The DOF is also affected, with DOF of $\left.f_{1}\right|_{17 \mathrm{~mm}}=24 \mathrm{~mm},\left.f_{1}\right|_{25 \mathrm{~mm}}=20 \mathrm{~mm}$, and $\left.f_{1}\right|_{33 \mathrm{~mm}}=16 \mathrm{~mm}$. The 
stand-off distance of $f_{1}=25 \mathrm{~mm}$ was chosen to obtain the best resolution and DOF.

(a) Image from experiment, $f_{1}=25 \mathrm{~mm}$

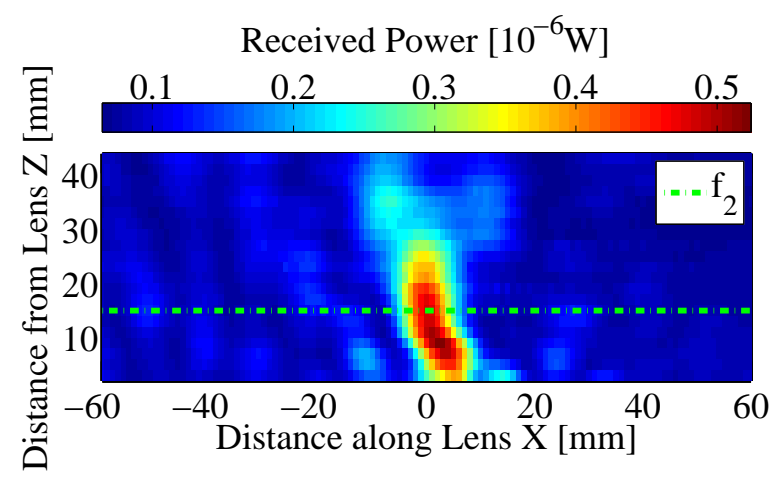

(c) Scan of the 2-D HFL imaged $\lambda$ aperture

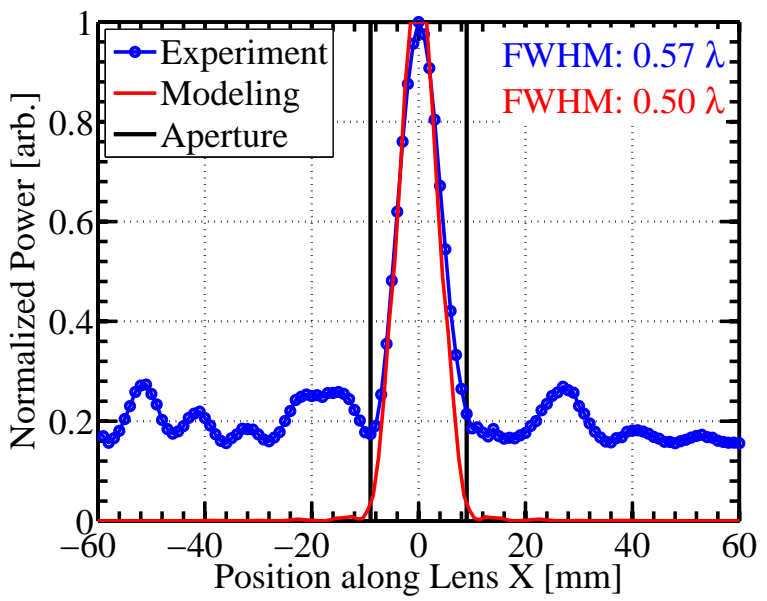

(b) Image from modeling, $f_{1}=25 \mathrm{~mm}$

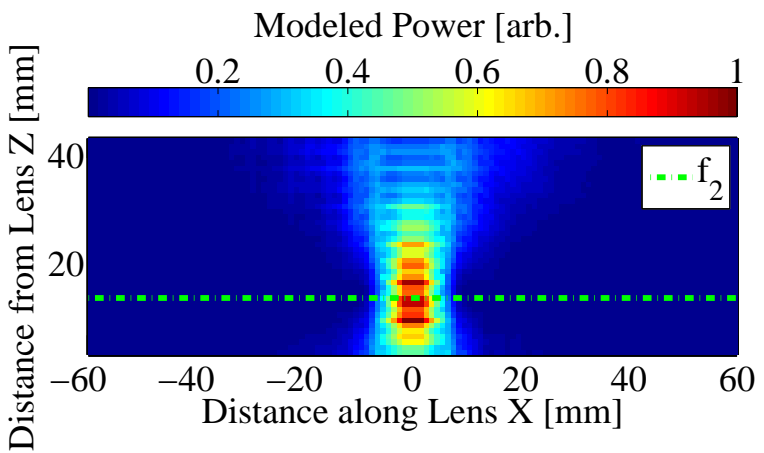

(d) Near-field scan of $\lambda$ aperture

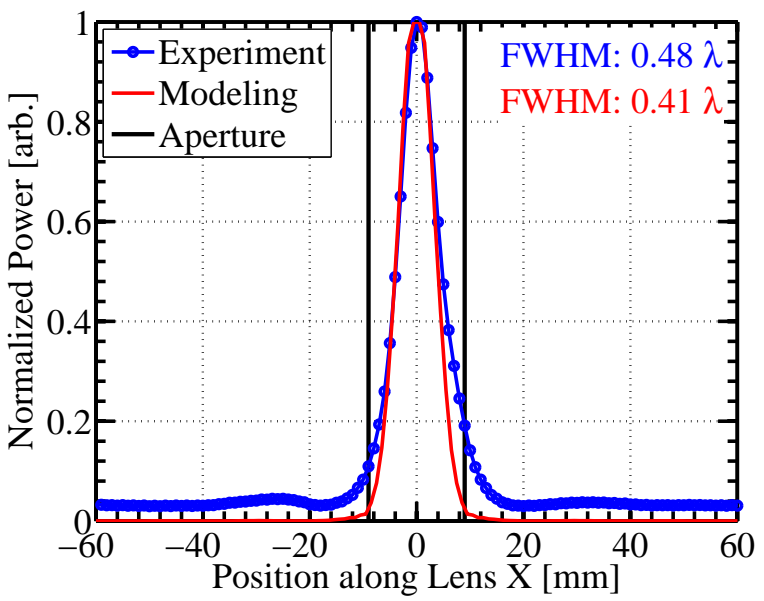

Figure 5.4: HFL transmission mode imaging comparison of modeling versus experiment and HFL versus near-field using a single aperture with $\lambda$ feature size and stand-off distance $f_{1}=25 \mathrm{~mm}$. (a) and (b) match well, the focal plane $f_{2}=15 \mathrm{~mm}$. (c) compares the experiment to modeling of the HFL image at $f_{2}$. The object to image distance for the HFL was $78 \mathrm{~mm}(4.32 \lambda)$ for experiment, and $77.5 \mathrm{~mm}(4.29 \lambda)$ for modeling. (d) presents the near-field image of the $\lambda$ sized aperture. The near-field measurement was taken at $3 \mathrm{~mm}$ $(0.17 \lambda)$ in front of the aperture and modeled data at the aperture.

Experiment and modeling results matched well, and the 2-D HFL resolved the $\lambda$ feature size aperture well (see fig. 5.4). A near-field scan of the aperture did outperform the HFL, however also required a much more stringent stand-off distance of $0.17 \lambda$ versus $4.32 \lambda$. As for dual apertures, the 2-D HFL was not able to resolve the dual aperture sample with $0.75 \lambda$ separation (see fig. 5.5a). Separate images of the left and right aperture resolved the 
individual aperture well. However, the image with both apertures open was not resolved. In contrast, a near-field scan of the same sample (fig. 5.5b) resolved the dual apertures at a stand-off distance of $f_{1}=0.17 \lambda$.

(a) Image of dual apertures, $0.75 \lambda$ separation

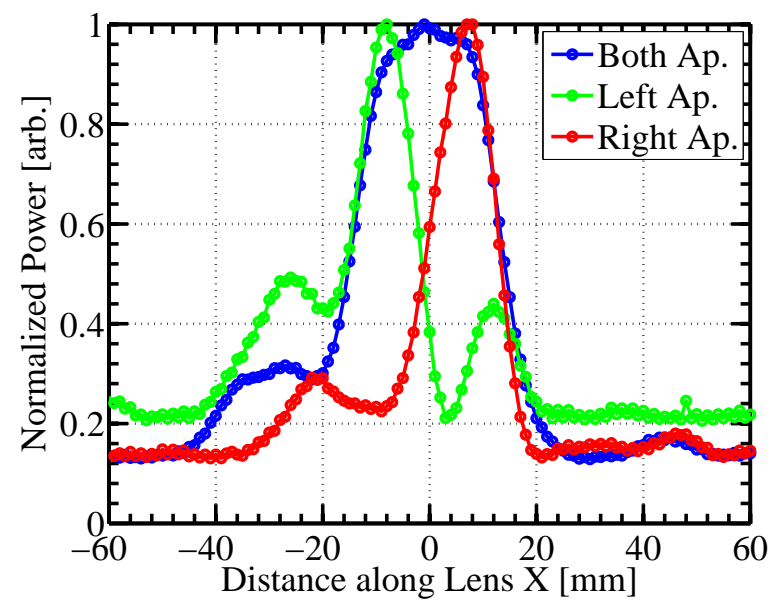

(b) Near-field scan vs HFL

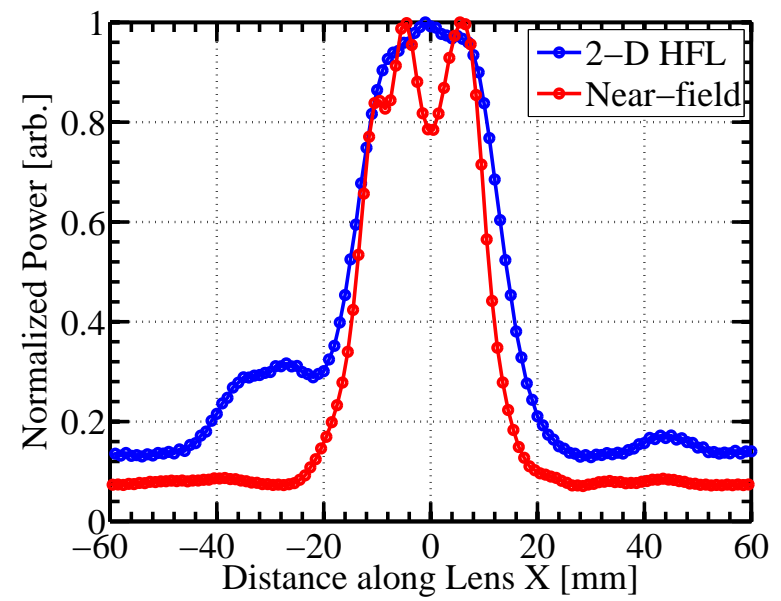

Figure 5.5: HFL transmission mode image of a dual aperture sample with $\lambda / 2$ feature size and $0.75 \lambda$ separation at $f_{1}=25 \mathrm{~mm}$. (a) The dual aperture image at the focus $\left(f_{2}=15 \mathrm{~mm}\right)$ of the HFL was not resolved, even though the individual left and right apertures were well imaged. (b) the near-field scan resolved the same dual aperture sample at $f_{1}=3 \mathrm{~mm}$.

A dual aperture with $\lambda$ separation was also measured and was well resolved (see fig. 5.6). However, the imaged apertures' locations were found to be slightly off from their physical location. This could be due to resolution limits of the lens as well as a slight magnification factor, owing to the HFL's $n_{\text {eff }}$.

Overall, the HFL's resolution was diffraction limited. However as compared to near-field measurements, the HFL lost $\approx 0.1 \lambda$ in resolution, while maintaining subwavelength resolution for object to image distance of $78 \mathrm{~mm}(4.3 \lambda)$. The resolution of the HFL in transmission mode imaging is tied to the FWHM. The smallest observed FWHM was $10.2 \mathrm{~mm}(0.57 \lambda)$ for $f_{1}=25 \mathrm{~mm}(1.38 \lambda)$ object to lens distance, with a DOF of $20 \mathrm{~mm}(1.11 \lambda)$.

In comparison to literature, the experiment fared well. The resolution of 2-D HFL was on the order of previously described values $[16,18,63]$. The $2-\mathrm{D}$ HFL reported in this dissertation, however, was much more practical than the lenses described previously $[16,63]$. 
(a) Image from Experiment

Received Power $[\mu \mathrm{W}]$

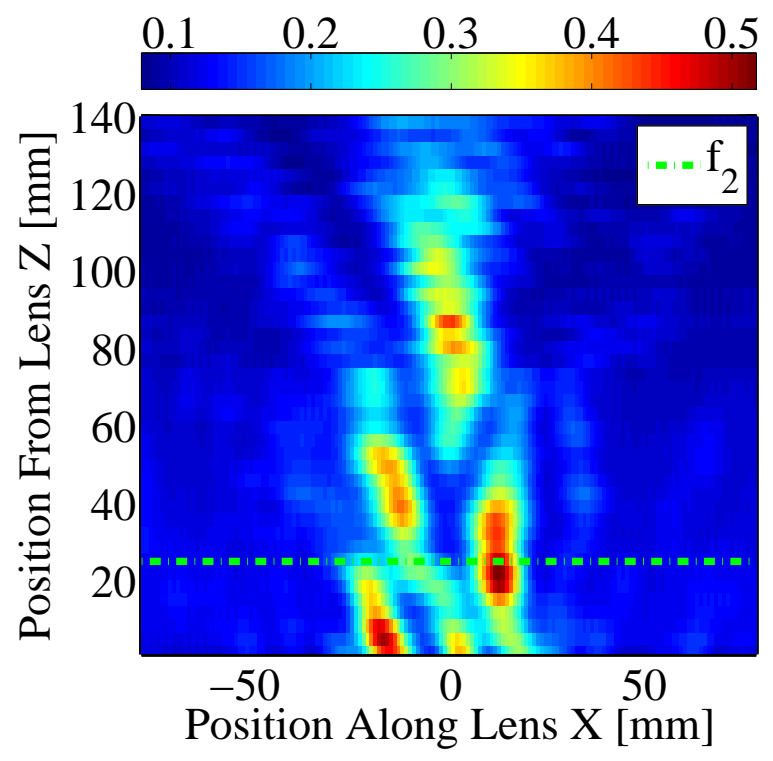

(c) Image of the dual aperture with the HFL

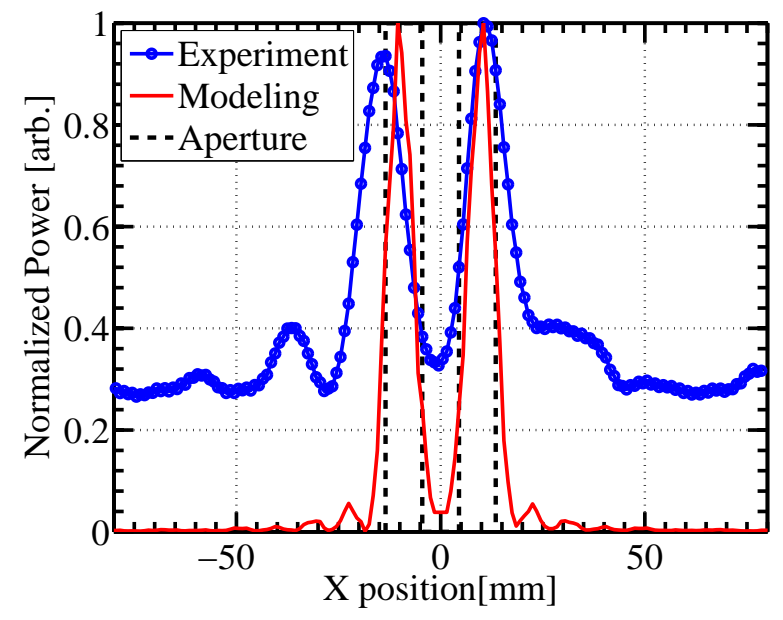

(b) Image from Modeling

Modeled Power [arb.]

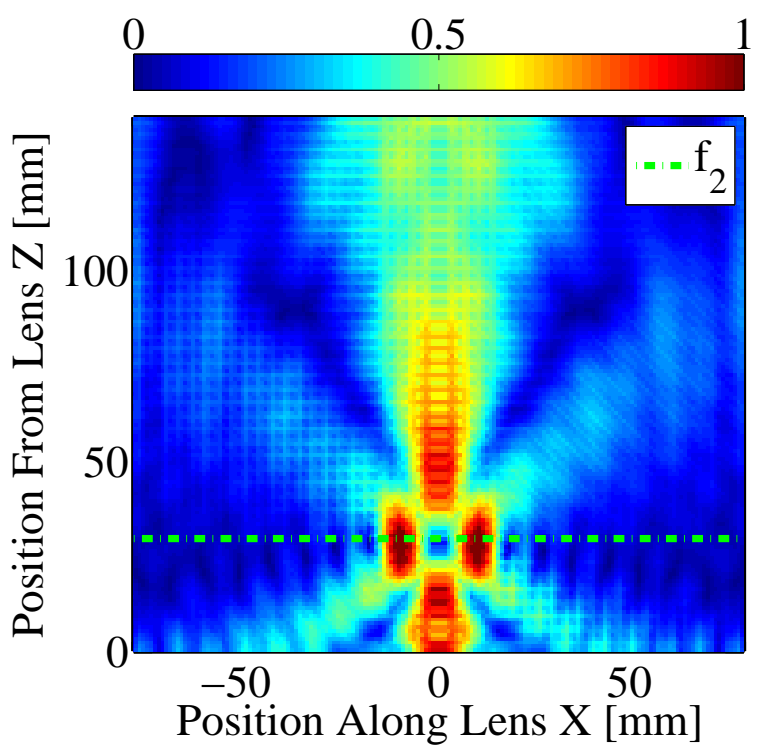

(d) Near-field scan of the dual aperture

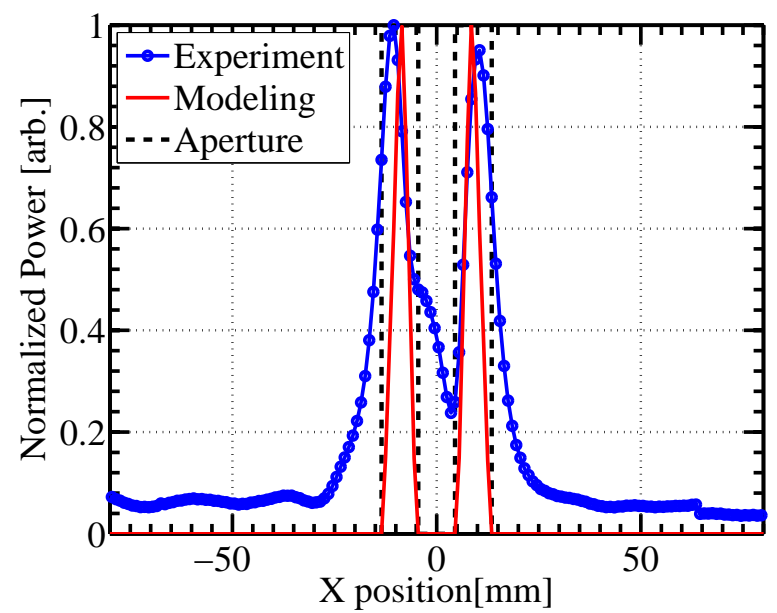

Figure 5.6: HFL transmission mode imaging characteristics of dual apertures. Experiment and modeling are compared for an imaged double $\lambda / 2$ aperture with $\lambda$ separation. Aperture to lens stand-off distance was set to $f_{1}=10 \mathrm{~mm}$. Experiment and modeling share similarities. (a) experiment exhibited $f_{2}=25 \mathrm{~mm}$ and (b) modeling showed $f_{2}=29.5 \mathrm{~mm}$. (c) Compares the imaged aperture from experiment, modeled results, and the physical aperture. (d) compares the results for the near-field scan. Both image and near-field scans show a well resolved aperture. For both modeling and experiment results show that the dual apertures are well resolved. 
Their experiments were conducted with very thin (in terms of $\lambda$ ) MTM lenses, rendering them impractical for actual imaging purposes due to very small standoff distance requirements. Furthermore, Aydin et al. [16] used two incoherent MW sources as image objects to overcome interference effects whereas the 2-D HFL experiment used a coherent source, as would be the case in a typical imaging application. 


\subsection{Reflection Mode Imaging - Single Detector}

\subsubsection{Single Detector High Frequency Lens}

The superior 2-D HFL was used for the reflection mode imaging experiment. In order to image, a MTM lens requires a continuous wave microwave source. Furthermore, the transmission losses in the HFL dictated a separate source and detector. Therefore, the reflection mode image was based on an interference image between the source antenna reflecting off of the sample and the direct path between the antenna and the detector. This resulted in a constructive and deconstructive interference pattern. The challenge was to align the source and detector in such a way that the constructive interference peak coincided at the focus of the MTM lens. By observing the pattern in the X-Z plane, aperture samples were imaged (fig. 5.7).

Fig. 5.7, shows the raw data from the interference pattern without a sample, the reference blank FR4 sample, and three aperture samples with $\lambda / 4, \lambda / 2$, and $\lambda$ feature size. It can be clearly seen that the apertures were present in the images. Line scans along the $\mathrm{x}$-axis at $\mathrm{z}=23 \mathrm{~mm}$ revealed the apertures even further (see fig. 5.8a). The line scan was then referenced to the blank sample and inverted to obtain the FWHM of the apertures and referenced power as a function of position along the lens fig. 5.8b. The reflection mode image revealed that the FWHM $(1.17 \lambda)$ has increased as compared to the transmission mode image $(0.57 \lambda)$ with the same lens.

While the FWHM is an indicator for obtainable resolution, it is of note that a dual aperture sample with $\lambda / 2$ feature size and $0.75 \lambda$ separation was resolved. Fig. 5.9 shows the raw data of a no sample condition, blank FR4 reference, open left aperture, open right aperture, both apertures open, and the line scan of the dual aperture sample. Further analysis (see fig. 5.10a) of the scan revealed that the aperture peak to peak distance was $13.2 \mathrm{~mm}$, and the trough between the peaks met the resolution criterion with 0.81 of peak maximum. Furthermore, comparisons between the dual aperture and scans with only the left 
or right aperture open (fig. 5.10b) demonstrated that the dual apertures were resolved at subwavelength resolution. In comparison to a near-field measurement at $f_{1}=3 \mathrm{~mm}$ stand-off distance, fig. 5.10c shows that the lens gives very similar image performance albeit at a much more practical stand-off distance of $f_{1}=24 \mathrm{~mm}$. A near-field measurement at a similar stand-off distance of $f_{1}=23 \mathrm{~mm}$ did not reveal the dual apertures (see fig. 5.10d). This was expected as evanescent waves decay at larger than $\lambda$ stand-off distances.

Table 5.1: Resolution of imaged samples. Single apertures were characterized for their FWHM. Dual apertures were characterized for their peak to peak separation and the trough between the peaks. Dual apertures were considered resolved, as per Rayleigh criterion, where the trough between the peaks was 0.81 of the peak maximum.

\begin{tabular}{|c|c|c|c|c|c|}
\hline \multirow{3}{*}{ 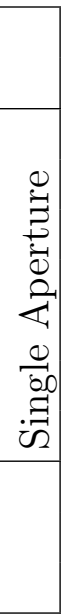 } & \multicolumn{3}{|c|}{$\begin{array}{l}\text { Sample } \\
\text { ture size }\end{array}$} & \multicolumn{2}{|c|}{$\begin{array}{l}\text { Image } \\
\text { FWHM }\end{array}$} \\
\hline & $\begin{array}{l}\text { Metal } \\
\text { Metal } \\
\text { Metal } \\
\text { FR4 } \\
\text { FR4 } \\
\text { FR4 }\end{array}$ & \multicolumn{2}{|c|}{$\begin{array}{l}4.5 \mathrm{~mm}(0.25 \lambda) \\
9 \mathrm{~mm}(0.5 \lambda) \\
18 \mathrm{~mm}(\lambda) \\
4.5 \mathrm{~mm}(0.25 \lambda) \\
9 \mathrm{~mm}(0.5 \lambda) \\
18 \mathrm{~mm}(\lambda)\end{array}$} & \multicolumn{2}{|c|}{$\begin{array}{l}30.7 \mathrm{~mm}(1.71 \lambda) \\
18.8 \mathrm{~mm}(1.04 \lambda) \\
15.9 \mathrm{~mm}(0.88 \lambda) \\
16.4 \mathrm{~mm}(0.91 \lambda) \\
16.9 \mathrm{~mm}(0.94 \lambda) \\
21.1 \mathrm{~mm}(1.17 \lambda)\end{array}$} \\
\hline & \multicolumn{3}{|c|}{$\begin{array}{l}\text { Sample } \\
\text { e size }\end{array}$} & $\begin{array}{l}\text { Ima } \\
\text { peak to peak } \\
\text { separation }\end{array}$ & $\begin{array}{l}\text { trough } \\
\text { norm. power }\end{array}$ \\
\hline 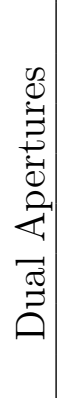 & $\begin{array}{l}\text { Metal } \\
\text { Metal } \\
\text { Metal } \\
\text { Metal } \\
\text { FR4 } \\
\text { FR4 } \\
\text { FR4 } \\
\text { FR4 }\end{array}$ & $\begin{array}{l}9 \mathrm{~mm}(0.5 \lambda) \\
9 \mathrm{~mm}(0.5 \lambda) \\
9 \mathrm{~mm}(0.5 \lambda) \\
9 \mathrm{~mm}(0.5 \lambda) \\
9 \mathrm{~mm}(0.5 \lambda) \\
9 \mathrm{~mm}(0.5 \lambda) \\
9 \mathrm{~mm}(0.5 \lambda) \\
9 \mathrm{~mm}(0.5 \lambda)\end{array}$ & $\begin{array}{l}13.5 \mathrm{~mm}(0.75 \lambda) \\
18 \mathrm{~mm}(\lambda) \\
27 \mathrm{~mm}(1.5 \lambda) \\
36 \mathrm{~mm}(2 \lambda) \\
13.5 \mathrm{~mm}(0.75 \lambda) \\
18 \mathrm{~mm}(\lambda) \\
27 \mathrm{~mm}(1.5 \lambda) \\
36 \mathrm{~mm}(2 \lambda)\end{array}$ & $\begin{array}{l}12.2 \mathrm{~mm}(0.68 \lambda) \\
23.1 \mathrm{~mm}(1.28 \lambda) \\
26.6 \mathrm{~mm}(1.48 \lambda) \\
37.3 \mathrm{~mm}(2.07 \lambda) \\
13.2 \mathrm{~mm}(0.73 \lambda) \\
22.9 \mathrm{~mm}(1.27 \lambda) \\
23.2 \mathrm{~mm}(1.29 \lambda) \\
38.1 \mathrm{~mm}(2.12 \lambda)\end{array}$ & $\begin{array}{l}0.85 \\
0.58 \\
0.06 \\
0.00 \\
0.80 \\
0.56 \\
0.25 \\
0.07\end{array}$ \\
\hline
\end{tabular}

Further sample information is provided in table 5.1. In the table it is of interest to note that the FWHM of the metal samples was erratic as compared to the FR4 samples. This is thought to be due to scattering effects on the aperture cutouts of the highly reflective sheet metal. Similar scattering was observed in the FR4 samples (5.8a), where for positive x, the scans show oscillations above and below the reference sample. The amplitude of these 
is not symmetric for negative $\mathrm{x}$ values due to a tilt in the sample. This was necessary to compensate for the inherent tilt in the image plane due to the asymmetric arrangement of the SRRs. The tilt exacerbated scattering from one side of the aperture. For the dual apertures, the correctional tilt lead to a slight discrepancy between the received power for the respective peaks, as one aperture was slightly closer to the source antenna than the other. The correctional tilt was $\approx 13^{\circ}$ of angle. The tilt in imaging can be seen in fig. 5.4a. For dual aperture samples, the metal and FR4 samples matched well with each other. Furthermore, the peak to peak distance in the image plane replicated the separation distance of the samples well, and all but one metal sample met the resolution criterion. The $0.75 \lambda$ separation sample was not resolved, most likely due to the high reflection coefficient of the sample compounded with the imaging tilt inherent to the MTM lens.

In reflection mode the HFL outperformed the transmission mode measurements (fig. 5.5) and revealed that the reflection mode image was comparable to the transmission mode near-field measurement at a much greater stand-off distance of $f_{1}=23 \mathrm{~mm}(1.27 \lambda)$ versus $f_{1}=3 \mathrm{~mm}(0.17 \lambda)$. The HFL provided for subwavelength resolution imaging with a total sample to image distance of $\sim 4 \lambda$.

The results show that the lens increased the resolution as compared to the no lens condition significantly, as well as compared well to the transmission mode near-field measurement. 
(a) No Sample

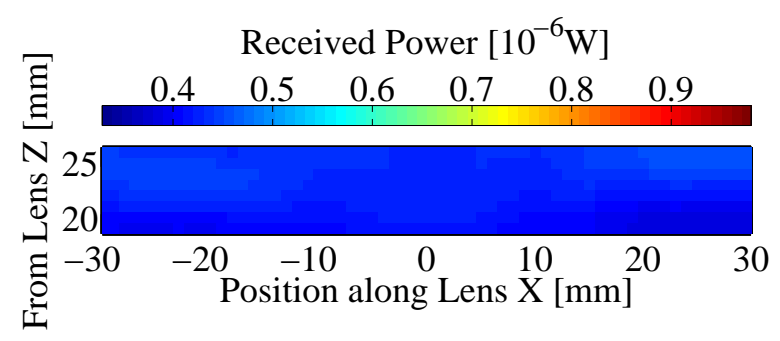

(c) Single Aperture, feature size $\lambda / 4$

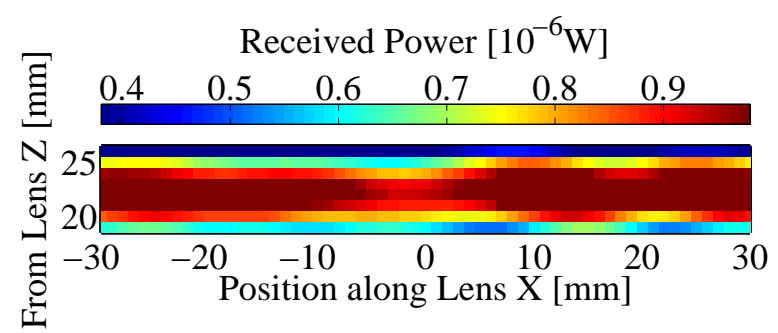

(b) Blank FR4

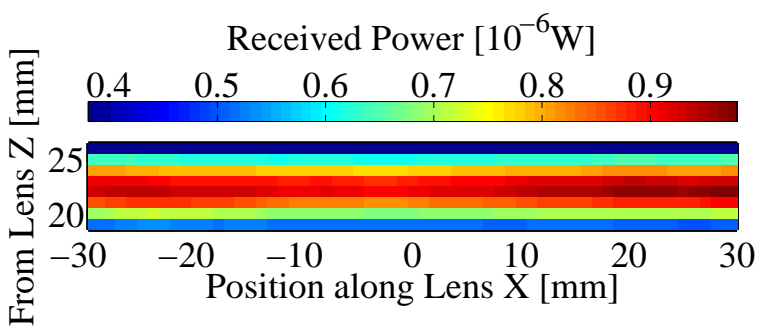

(d) Single Aperture, feature size $\lambda / 2$

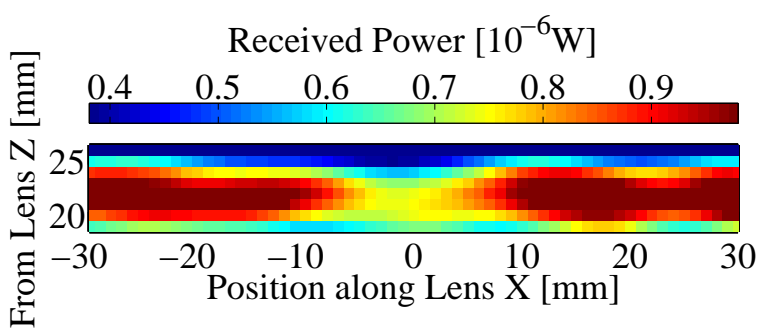

(e) Single Aperture, feature size $\lambda$

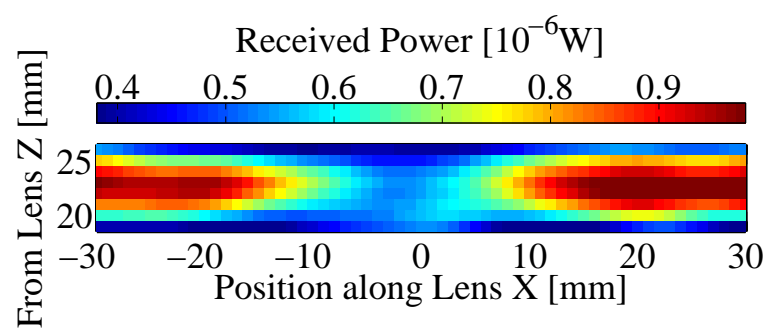

Figure 5.7: HFL reflection mode imaging of single apertures. The HFL image is evaluated with an empty sample holder (a), a blank FR4 sample (b), and single aperture samples made of FR4 with different feature sizes: $\lambda / 4$ (c), $\lambda / 2(\mathrm{~d})$, and $\lambda(\mathrm{e})$. In reflection mode, the sample was stepped along the $\mathrm{x}$ and $\mathrm{z}$ axis, where the image underwent constructive and deconstructive interference along the z-axis. In this reflection mode, empty space is indicated by a low reflection value (blue color) and presence of a material as high reflection (red color). The focus was set at the maximum received signal of the blank sample, at $f_{1}=24 \mathrm{~mm}$ $(1.33 \lambda)$. 
(a) Scanned aperture $(\lambda / 2)$ and reference samples

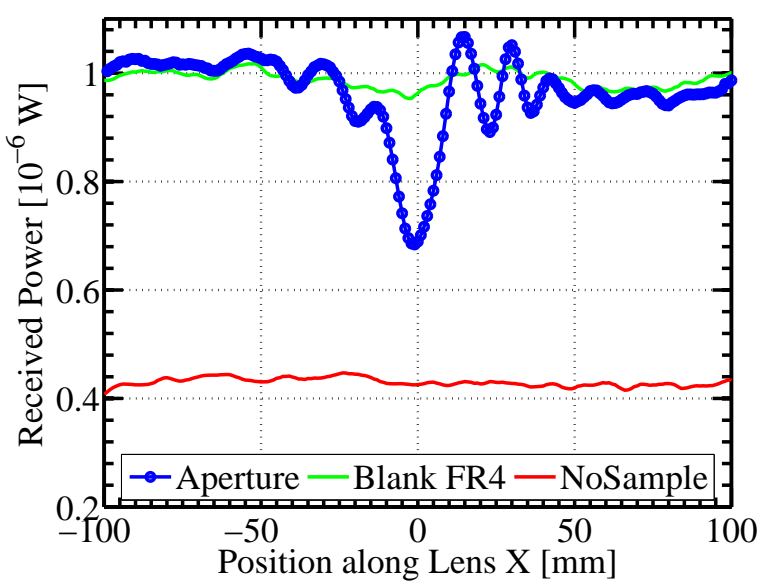

(b) Scanned single apertures

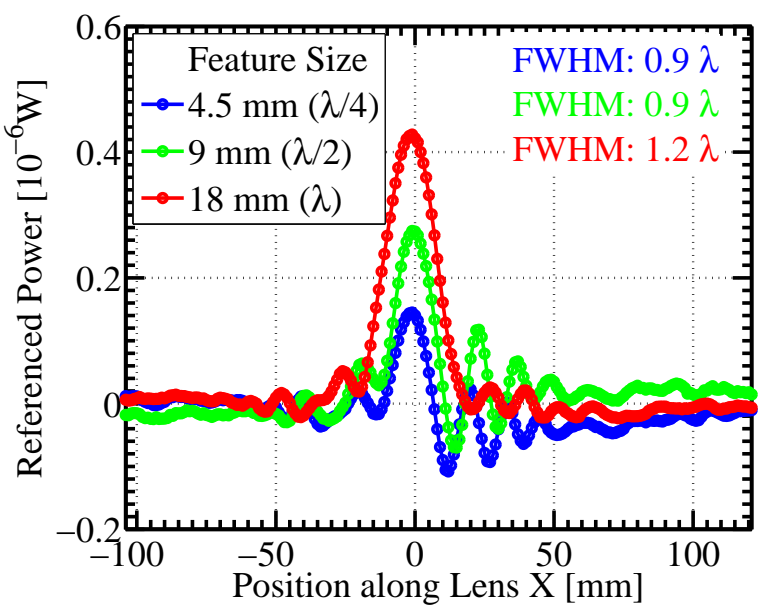

Figure 5.8: HFL reflection mode imaging characteristics of single apertures. The imaged sample was referenced to the blank FR4 measurements (a) by subtracting the blank FR4 information from the sample information and subsequently inverting the data (b). From this, the FWHM could be extracted. The FWHM decreases with decreasing feature size, as the aperture becomes less pronounced. The focus was set at $f_{1}=24 \mathrm{~mm}(1.33 \lambda)$ 
(a) No sample

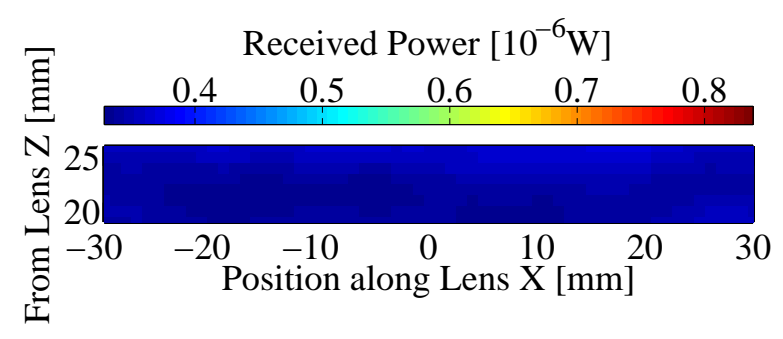

(c) Left aperture open

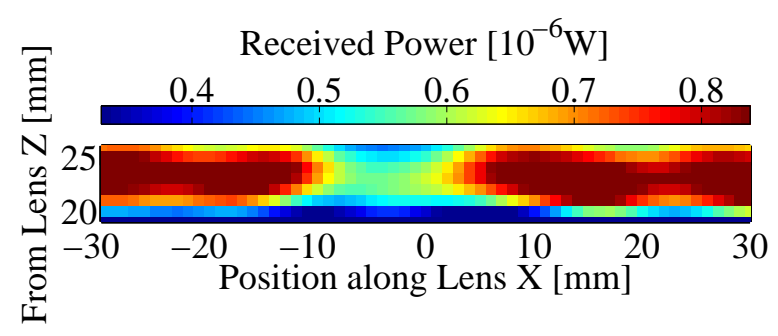

(e) Both apertures open

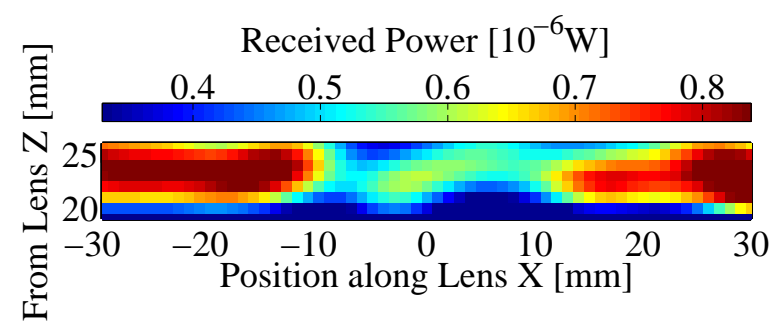

(b) Blank FR4

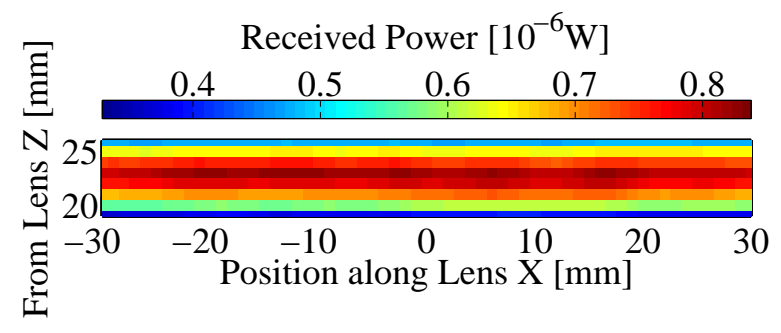

(d) Right aperture open

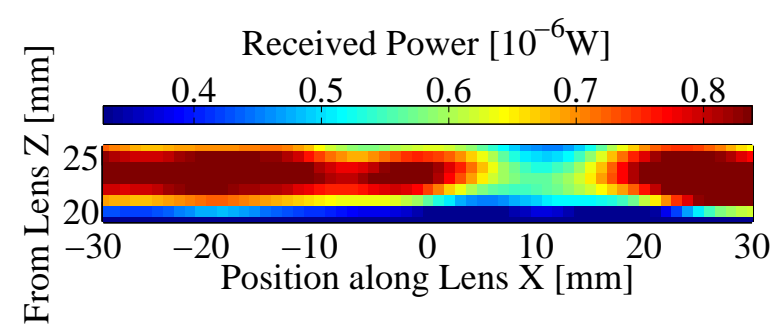

(f) Scan of dual aperture

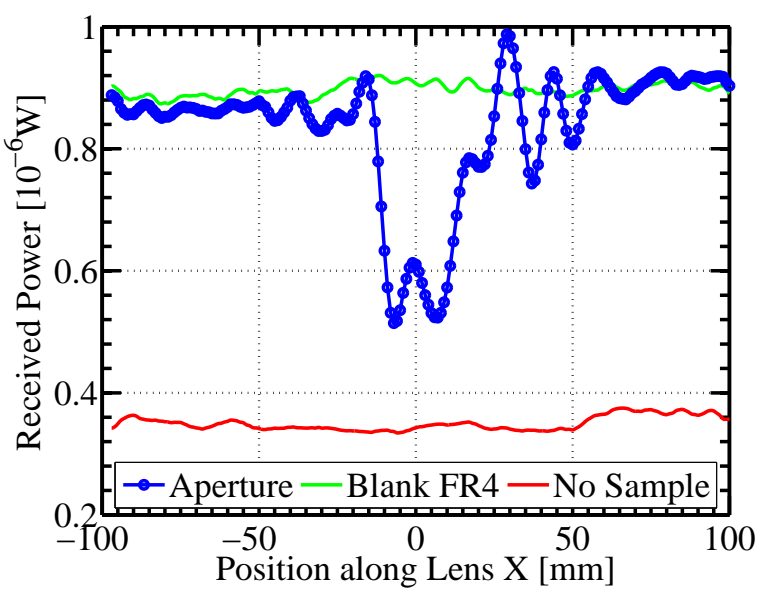

Figure 5.9: HFL reflection mode imaging of dual apertures. The imaging characteristics were evaluated with an empty sample holder (a), a blank FR4 sample (b), and dual aperture sample made of FR4 (e). The dual aperture had a feature size of $\lambda / 2$ and a separation distance of $0.75 \lambda$. The HFL was tested for its resolution by imaging the left aperture (c), the right aperture (d), and subsequently both open apertures (e). A line scan (f) of the dual aperture sample shows the received data in reference to the blank FR4 and empty sample holder (No Sample). The front focal length is $f_{1}=24 \mathrm{~mm}(1.33 \lambda)$ and lens to detector distance is $f_{2}=8 \mathrm{~mm}(0.44 \lambda)$. Figure reproduced with permission of author [71]. 
(a) Normalized line scan of dual aperture

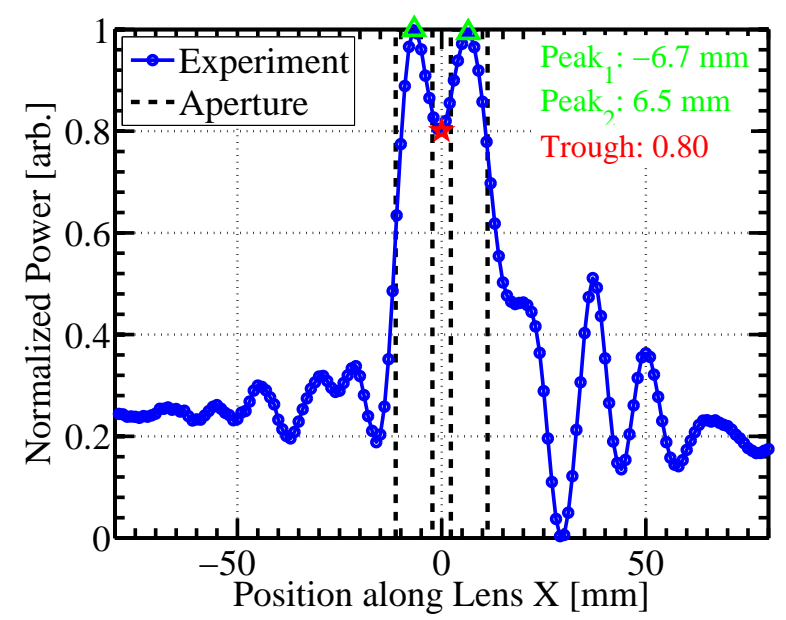

(c) Reflection mode near-field scan without lens

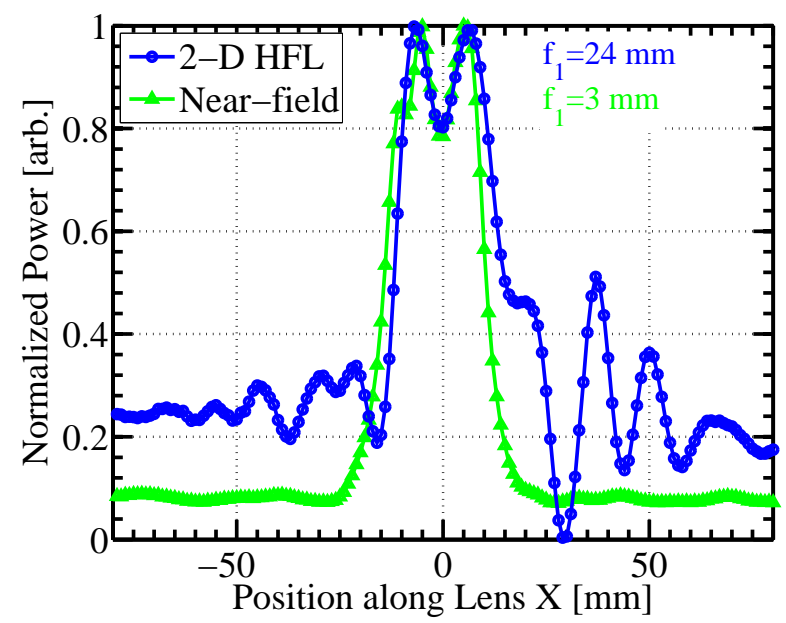

(b) Dual aperture resolution

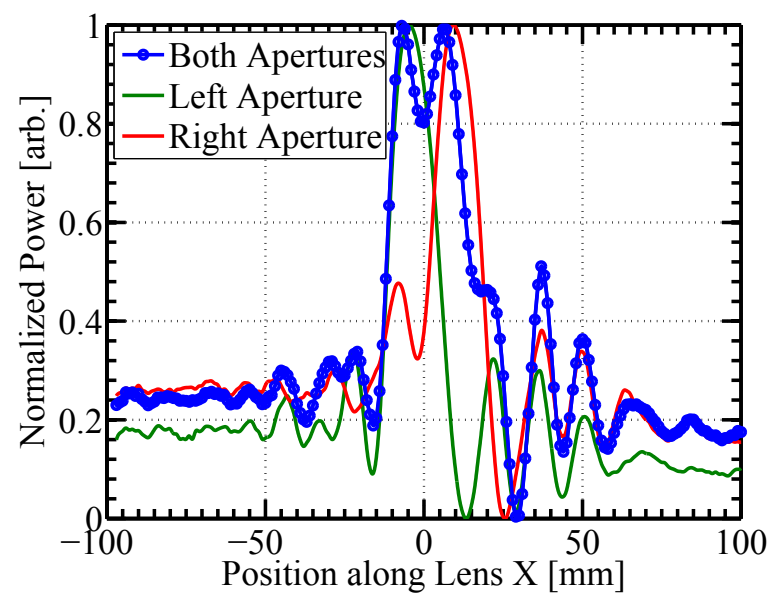

(d) Scan without lens

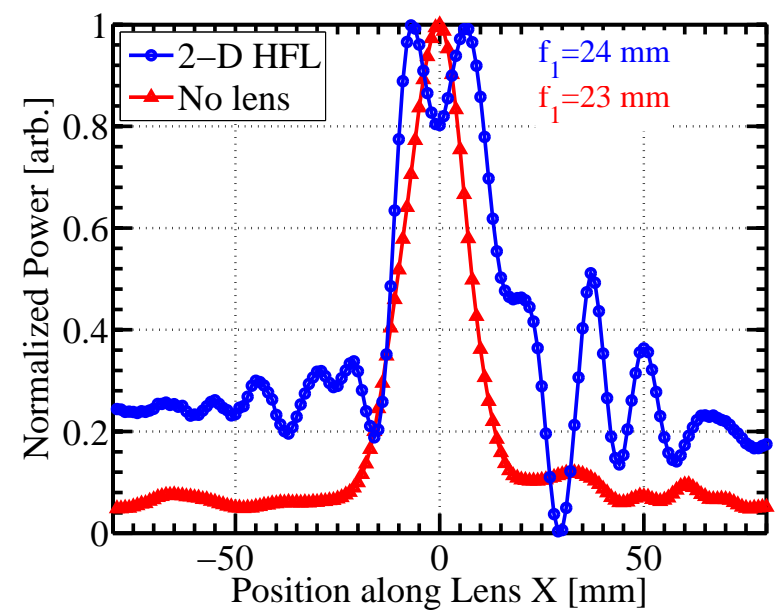

Figure 5.10: HFL reflection mode imaging resolution of dual apertures. (a) presents the scan of a dual aperture with $\lambda / 2$ slits and $0.75 \lambda$ separation. The dashed aperture lines denote the relative position of the apertures in of the physical sample. The trough of 0.80 peak maximum indicated that the apertures were resolved, and the peak to peak separation of $13.2 \mathrm{~mm}$ matches the physical sample separation of $13.5 \mathrm{~mm}$ very well. (b) line scan of the dual aperture image is presented along with the line scan of the individually resolved left and right aperture. The HFL's ability to image at subwavelength resolution in reflection mode was compared to a scan with the lens along the near-field of the aperture (c) as well as a scan of the aperture at a similar stand-off distance (d). The near-field scan and scan without lens were conducted in transmission mode. (a) and (b) reproduced with permission of author [71]. 


\subsubsection{Hidden Object Imaging}

The detection of hidden objects shows the proof of concept of applying MTMs for subwavelength resolution imaging for NDE applications. The reflection mode imaging technique proved promising as it was able to discern between slits of the "blind" dual aperture (fig. 5.11d). As compared to the regular scan, the hidden aperture signal showed slightly less dynamic range (fig. 5.11c). However, this was expected due to the extra sheet of FR4. The experiment showed that the reflection mode imaging technique was capable of resolving both hidden and exposed subwavelength sized features.

(a) Reference blank FR4

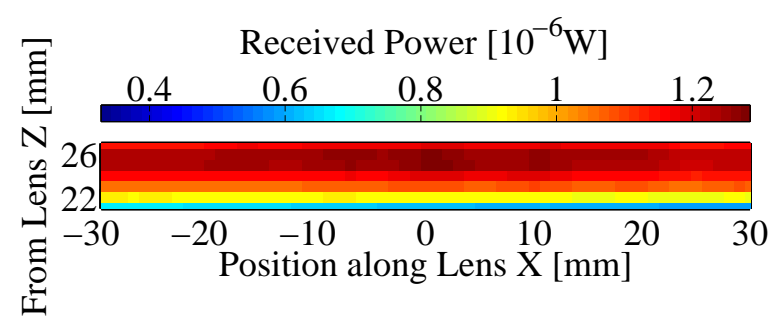

(c) Line scan

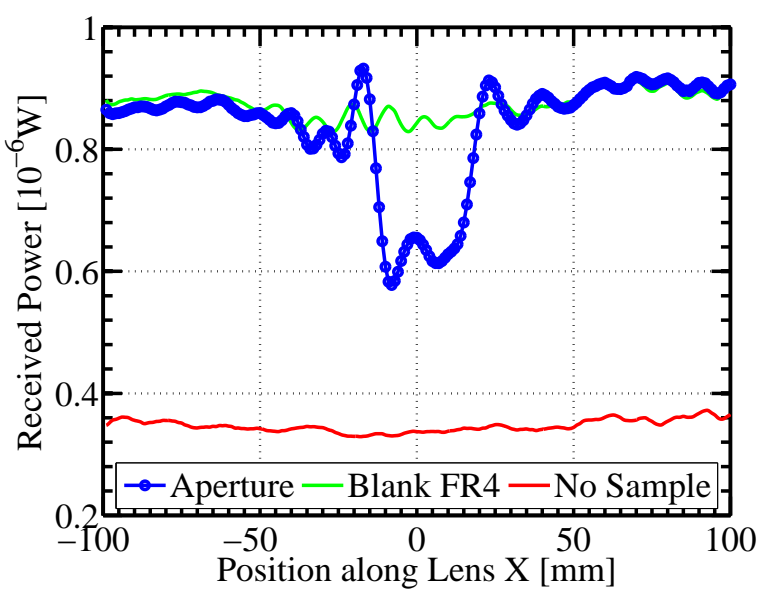

(b) Blind dual apertures

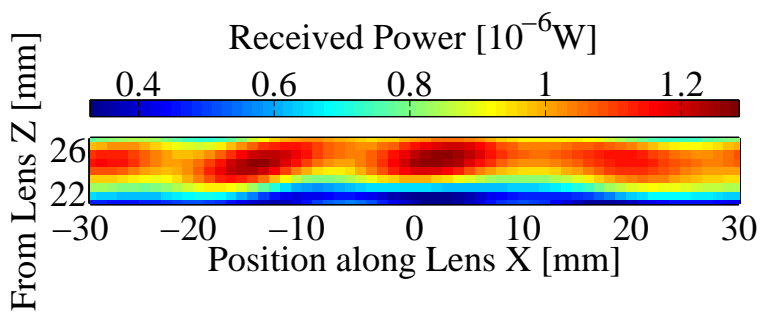

(d) Resolution of blind dual aperture

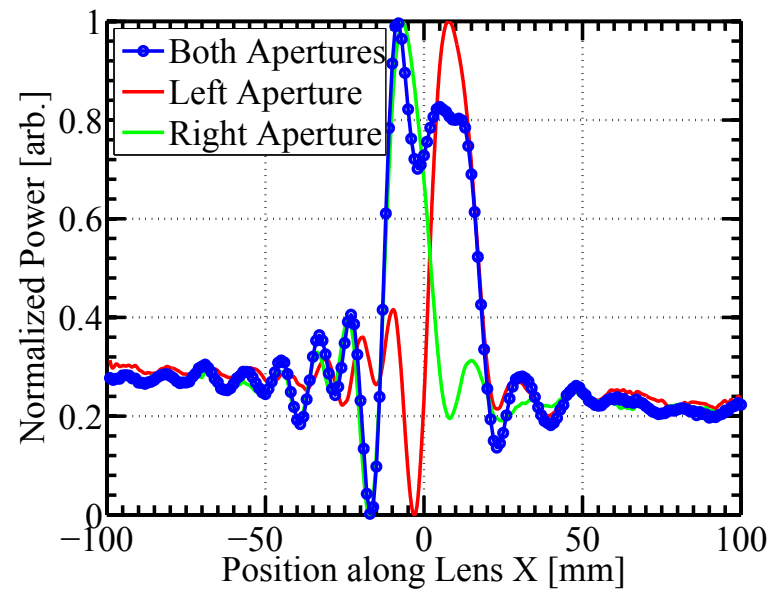

Figure 5.11: HFL imaging of hidden dual apertures with $\lambda / 2$ feature size and $0.75 \lambda$ separation made of FR4. (a) shows the image of the blank FR4 reference. (b) is the image of the hidden aperture. (c) is the line scan of the hidden aperture and (d) compares the resolved dual aperture to the individually imaged left and right aperture.

The main purpose of the reflection mode MTMs based MW imaging system was to 
establish an imaging technique capable of delivering 2-D images of hidden artifacts. The test was to evaluate the resolution capabilities, detection sensitivity, and the application of polarization rotation for imaging. Resolution capabilities were evaluated by measuring blind dual holes of different separation. Like the single and dual aperture study, baseline blank FR4 was used as a reference in the place of the sample. Fig. 5.12 shows the 2-D image of

(a) Blank FR4

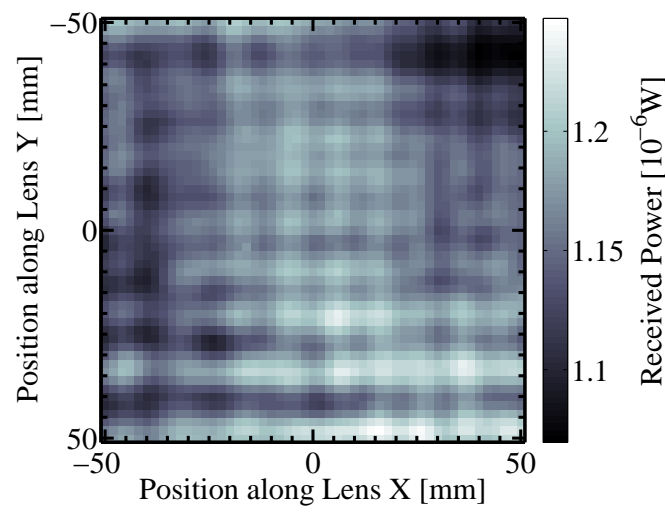

(c) Corrected image

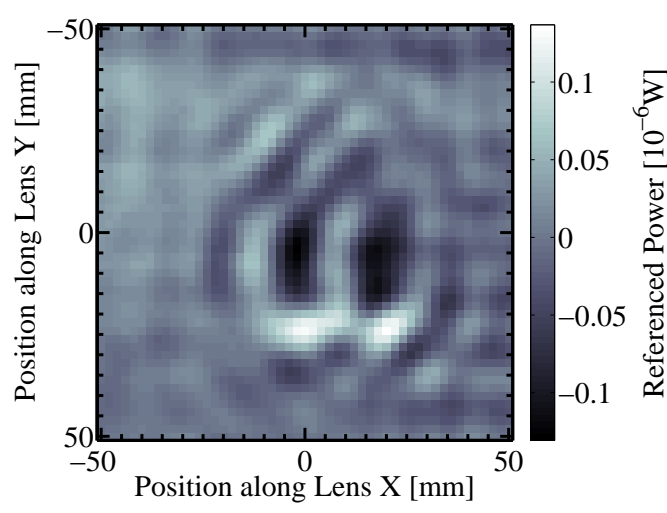

(b) Dual holes

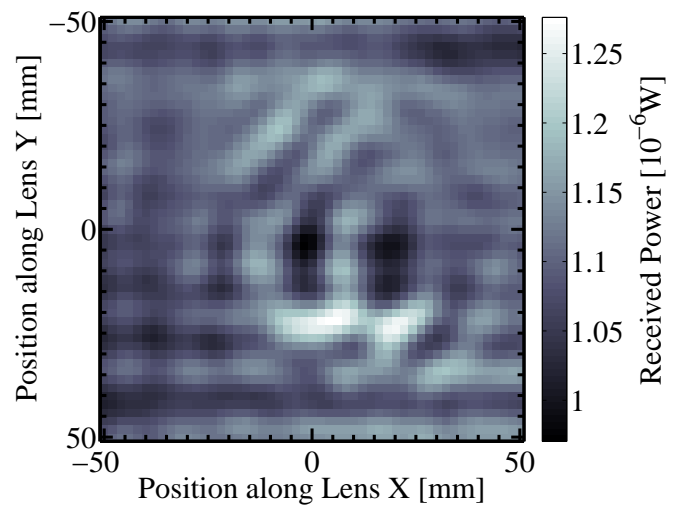

(d) Normalized and inverted line scan

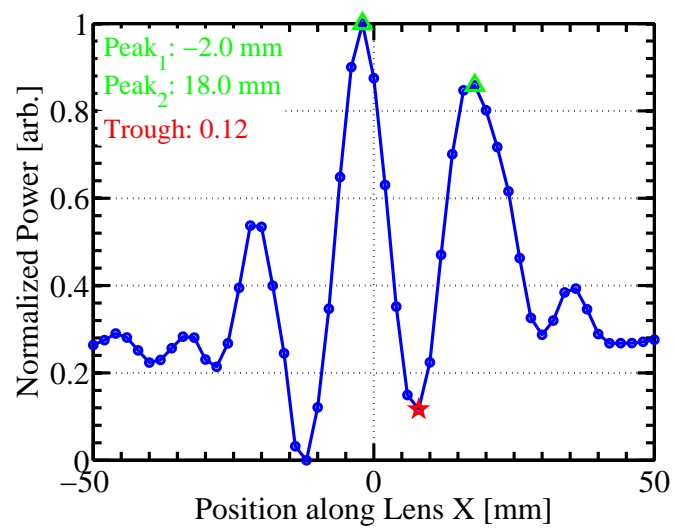

Figure 5.12: 2-D imaging of hidden dual holes. The hidden holes had $\lambda / 2$ diameter and $\lambda$ separation. The reflection mode image was obtained by scanning the sample in the $x-y$ plane at $f_{1}=26 \mathrm{~mm}$. The reference blank FR4 (a) is subtracted from the raw sample data (b), to obtain the referenced and corrected image (c). (d) normalized and inverted line scan of the referenced data. The dual holes were fully resolved and the peak to peak distance matched the hole separation well. Figure reproduced with permission of author [71].

$\lambda / 2$ holes with $\lambda / 2$ separation. The image shows the $\mathrm{x}-\mathrm{y}$ plane at a stand-off distance of $f_{1}=26 \mathrm{~mm}(1.44 \lambda)$. Data was taken in $2 \mathrm{~mm}$ by $2 \mathrm{~mm}$ intervals. The raw data of the 
blank FR4 (fig. 5.12a) is subtracted from the measured sample (fig. 5.12b), to obtain the referenced image (fig. 5.12c). The grid-line pattern in the blank FR4 sample stems from an interference effect between the continuous wave illumination of the source and the sample. By subtracting the reference from the sample measurement, the image from the holes is further enhanced. The imaged hole patterns are elongated in nature due to the 2-D HFL acting as a cylindrical lens exhibiting focusing along the $\mathrm{x}$-axis but not along the $\mathrm{y}$-axis. The diffracted pattern is due the diffraction phenomena present in the near-field of the imaged sample. Fig. 5.12d shows the normalized and inverted line scan across the holes. Here the two peaks are indicated to have a peak to peak distance of $20 \mathrm{~mm}$ and normalized power of 012 at the trough. This image is considered well resolved and the peak to peak distance was close to the actual separation distance of $18 \mathrm{~mm}$. Fig. 5.13 shows the procession of images for successively shorter separation while maintaining the feature size of the hole at $\lambda / 2$. The figure shows that the limit of resolution of the system is near a separation distance of $12 \mathrm{~mm}$ $(0.66 \lambda)$. Exact parameters are tabulated in table 5.2.

Table 5.2: Resolution and sensitivity results from the reflection mode imaging of the various samples. The imaging system was sensitive to $0.12 \lambda$ feature size and resolved dual holes with $0.66 \lambda$ separation. Table reproduced with permission of author [71].

\begin{tabular}{|c|c|c|c|c|c|}
\hline & \multicolumn{3}{|c|}{$\begin{array}{l}\text { Sample } \\
\quad \text { feature size }\end{array}$} & \multicolumn{2}{|l|}{$\begin{array}{c}\text { Image } \\
\text { FWHM }\end{array}$} \\
\hline \multirow[t]{2}{*}{ 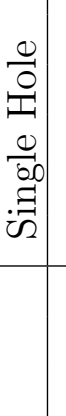 } & $\begin{array}{l}\text { FR4 } \\
\text { FR4 } \\
\text { FR4 } \\
\text { FR4 } \\
\text { FR4 }\end{array}$ & \multicolumn{2}{|c|}{$\begin{array}{l}5.9 \mathrm{~mm}(0.33 \lambda) \\
4.6 \mathrm{~mm}(0.26 \lambda) \\
3.3 \mathrm{~mm}(0.18 \lambda) \\
2.1 \mathrm{~mm}(0.12 \lambda) \\
1.0 \mathrm{~mm}(0.06 \lambda)\end{array}$} & $\begin{array}{l}14.7 \mathrm{~mm}(0 . \\
13.7 \mathrm{~mm}(0 . \\
15.3 \mathrm{~mm}(0 . \\
14.5 \mathrm{~mm}(0 . \\
15.4 \mathrm{~mm} \mathrm{(0.}\end{array}$ & $\begin{array}{l}32 \lambda) \\
6 \lambda) \\
55 \lambda) \\
31 \lambda) \\
55 \lambda)\end{array}$ \\
\hline & \multicolumn{3}{|c|}{\begin{tabular}{l}
\multicolumn{1}{c}{ Sample } \\
ature size
\end{tabular}} & $\begin{array}{l}\text { Image } \\
\text { peak to peak } \\
\text { separation }\end{array}$ & $\begin{array}{l}\text { trough } \\
\text { power }\end{array}$ \\
\hline 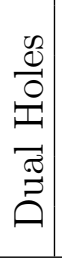 & $\begin{array}{l}\text { FR4 } \\
\text { FR4 } \\
\text { FR4 } \\
\text { FR4 } \\
\text { FR4 } \\
\end{array}$ & $\begin{array}{l}9 \mathrm{~mm}(0.5 \lambda) \\
9 \mathrm{~mm}(0.5 \lambda) \\
9 \mathrm{~mm}(0.5 \lambda) \\
9 \mathrm{~mm}(0.5 \lambda) \\
9 \mathrm{~mm}(0.5 \lambda)\end{array}$ & $\begin{array}{l}27 \mathrm{~mm}(1.5 \lambda) \\
18 \mathrm{~mm}(\lambda) \\
15 \mathrm{~mm}(0.83 \lambda) \\
12 \mathrm{~mm}(0.66 \lambda) \\
10 \mathrm{~mm}(0.56 \lambda)\end{array}$ & $\begin{array}{c}30 \mathrm{~mm}(1.67 \lambda) \\
20 \mathrm{~mm}(1.11 \lambda) \\
18 \mathrm{~mm}(1.00 \lambda) \\
16 \mathrm{~mm}(0.89 \lambda) \\
\text { Not Resol }\end{array}$ & $\begin{array}{r}0.00 \\
0.12 \\
0.26 \\
0.58 \\
\text { ed }\end{array}$ \\
\hline
\end{tabular}


(a) Separation $1.5 \lambda$

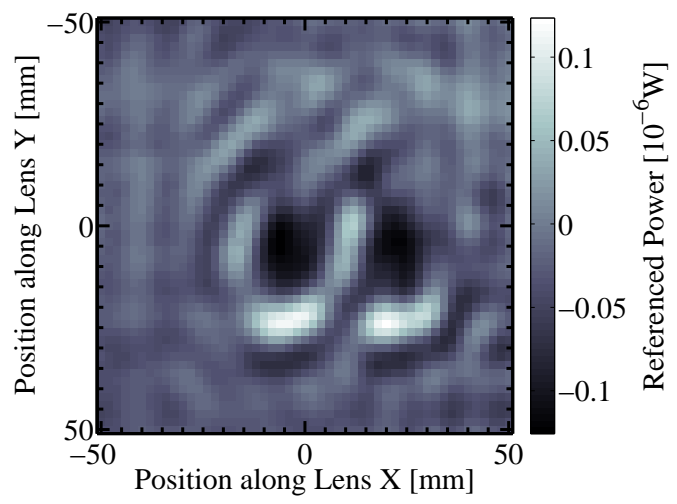

(c) Separation $0.66 \lambda$

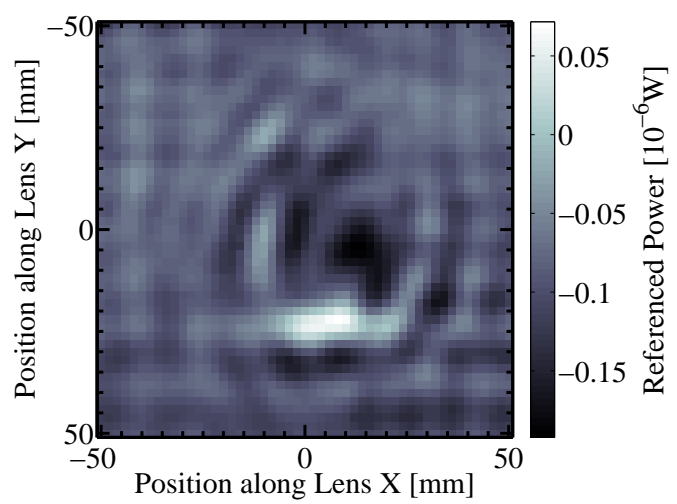

(b) Separation $0.83 \lambda$

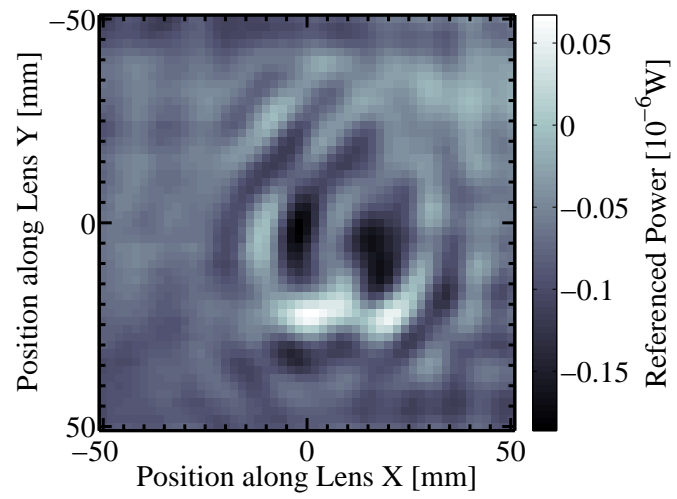

(d) Separation $0.56 \lambda$

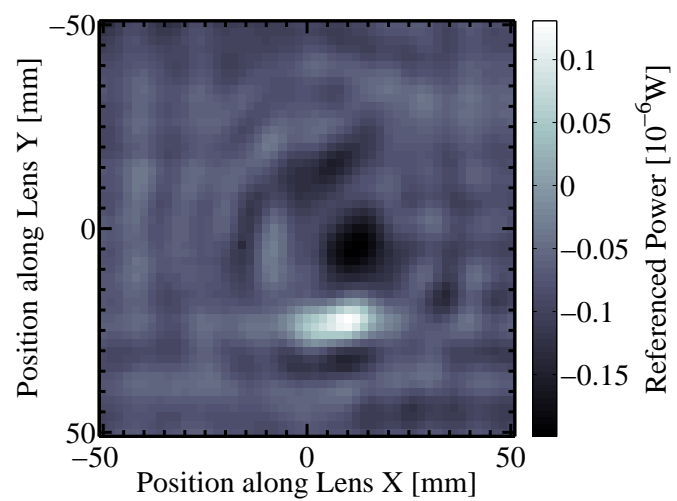

Figure 5.13: Referenced 2-D images of dual blind holes with various separation. These images show that the dual holes are distinguishable from each other and resolved down to a $0.66 \lambda$ separation as shown in (c). This established the resolution limit of the 2-D HFL in reflection mode.

Single holes of different sizes were used for the sensitivity measurements. The sample was scanned in the X-Y plane in $1 \mathrm{~mm}$ by $1 \mathrm{~mm}$ intervals at a stand-off distance of $f_{1}=26 \mathrm{~mm}$ $(1.44 \lambda)$. As in the case of the dual hole samples, the blank FR4 reference was measured first (fig. 5.14a) and then subtracted from the sample (fig. 5.14b) to arrive at the referenced image (fig. 5.14c). Fig. 5.14d shows the referenced power (reference subtracted from the sample and inverted) versus position along the $\mathrm{x}$-axis for various hole diameters. As the hole diameter diminishes, so does the imaged signal. Note that for the finest hole sizes the maximum deflection from the reference is $0.015 \times 10^{-6} \mathrm{~W}$, with the noise variation of referenced samples at $\pm 0.01 \times 10^{-6} \mathrm{~W}$. Therefore, it was concluded that the measurement system was sensitive 
(a) Reference blank FR4

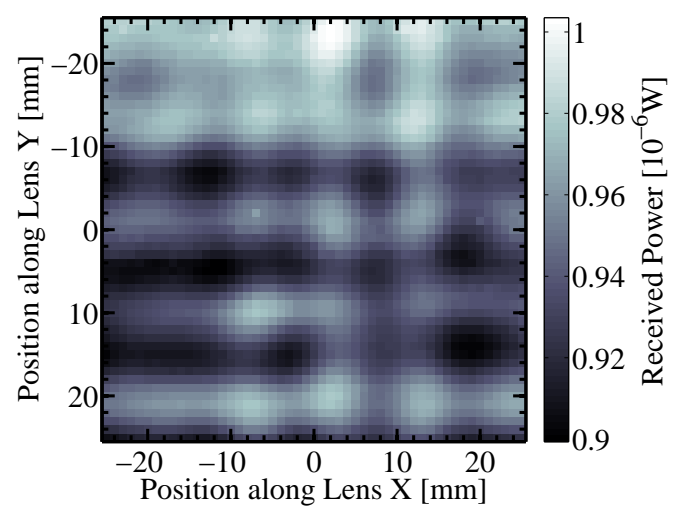

(c) Corrected image

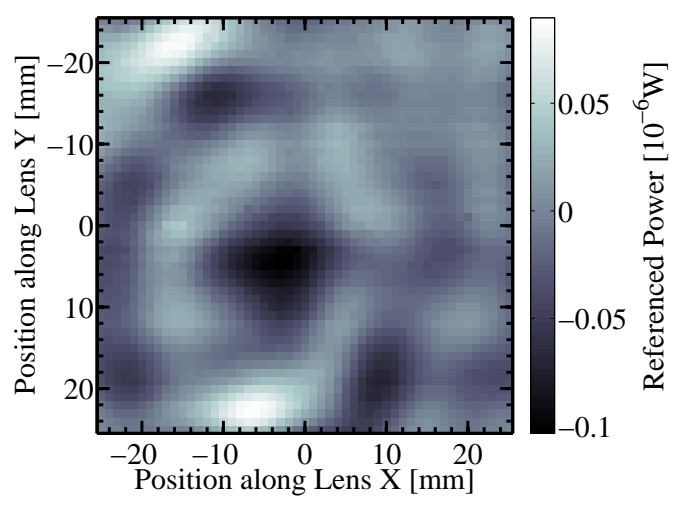

(b) FR4 single $5.9 \mathrm{~mm}$ hole

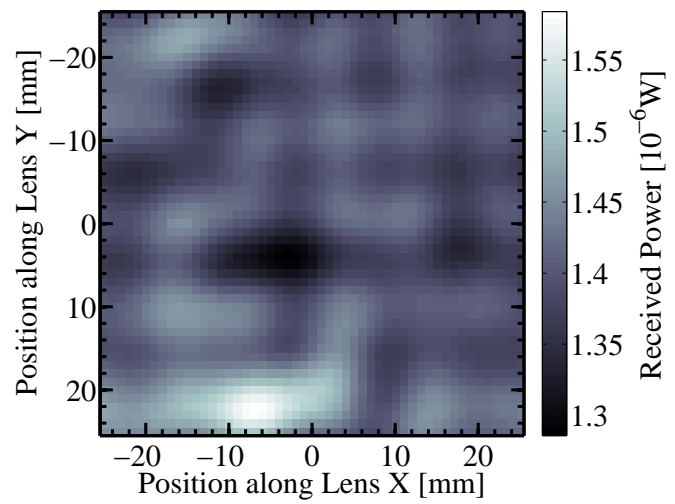

(d) Relative power of referenced holes

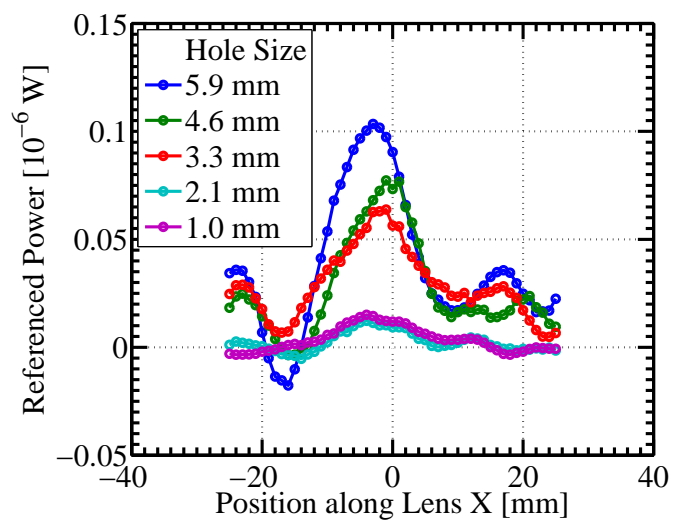

Figure 5.14: 2-D image of a hidden hole with $\lambda / 3$ feature size. The 2-D image was obtained by scanning the sample in the $\mathrm{x}-\mathrm{y}$ plane at $f_{1}=26 \mathrm{~mm}$. The reference blank FR4 (a) was subtracted from the raw sample data (b), to obtain the corrected image (c). (d) referenced power vs $\mathrm{x}$ position of the various single holes. The subwavelength sized holes were used to establish the sensitivity of the HFL based reflection mode imaging system. Based on the data, the $2.1 \mathrm{~mm}$ and $1 \mathrm{~mm}$ sized holes were no longer distinguishable from each other, while being slightly above the referenced noise-level. Sensitivity is reported as $2.1 \mathrm{~mm}(0.12 \lambda)$. Figure reproduced with permission of author [71].

to $2.1 \mathrm{~mm}(0.12 \lambda)$ features.

Fig. 5.15 shows the referenced images of the blind holes used for the sensitivity study. The $5.9 \mathrm{~mm}$ diameter (fig. 5.14c), $4.6 \mathrm{~mm}$ (fig. 5.15a), and $3.3 \mathrm{~mm}$ (fig. 5.15b) blind holes were distinguishable. The smallest two holes of $2.1 \mathrm{~mm}$ (fig. $5.15 \mathrm{c}$ ) and $1.0 \mathrm{~mm}$ (fig. 5.15d) diameter were indistinguishable from each other and barely above the noise-level. The FWHM data for the holes is presented in table 5.2. 
(a) Referenced image of $0.26 \lambda$ hole

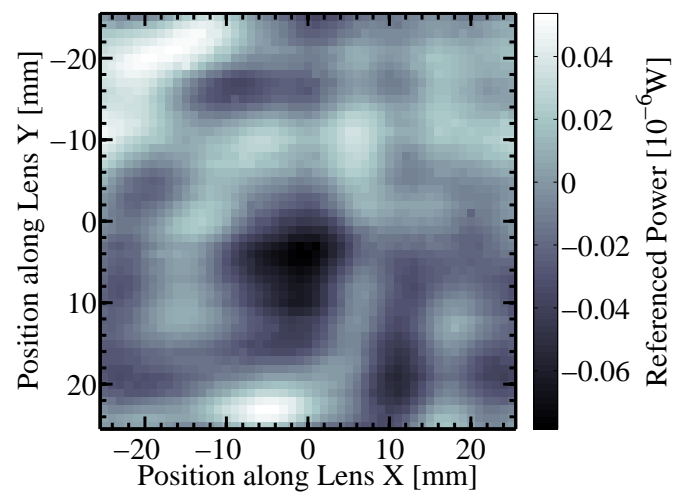

(c) Referenced image of $0.12 \lambda$ hole

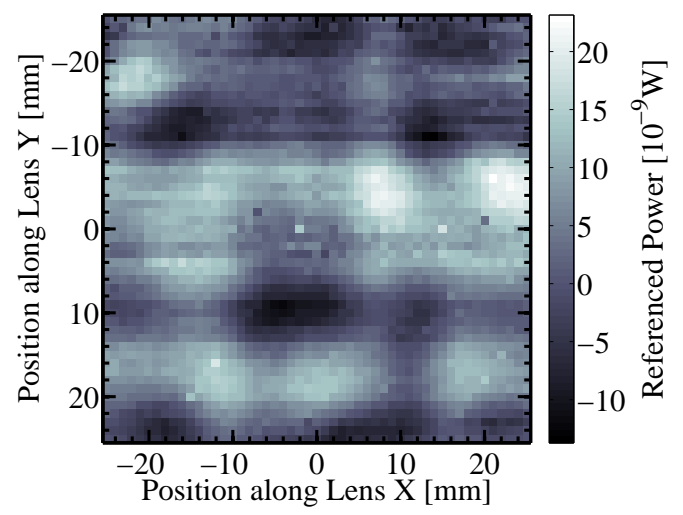

(b) Referenced image of $0.18 \lambda$ hole

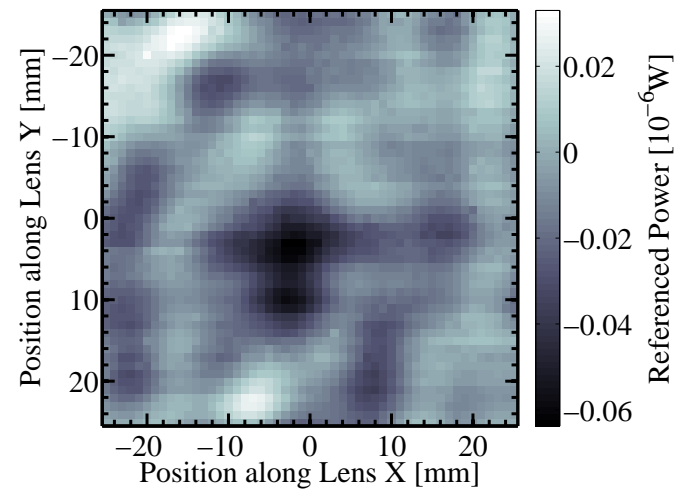

(d) Referenced image of $0.06 \lambda$ hole

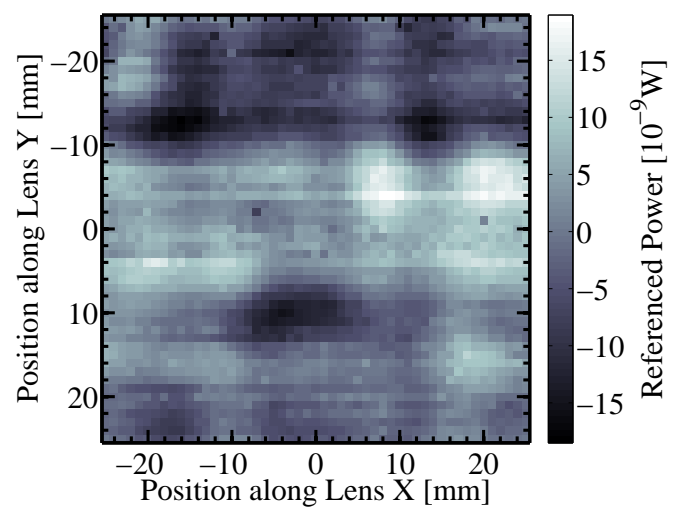

Figure 5.15: Referenced 2-D images of various sized hidden holes. The smallest two holes $(0.12 \lambda$ and $0.06 \lambda)$ were barely distinguishable from each other and the reference FR4.

The imaging concept was taken a step further towards demonstrating subwavelength 2-D MW imaging capabilities by imaging hidden "UVA" letters as shown in Fig.5.16a. The sample was resolved and the letters are legible (fig. 5.16b). While these features were not spaced at subwavelength intervals, the experiment showcased the ability of MTM lenses to image 2-D objects. This study presented the first report of $2-\mathrm{D}$ images obtained with a reflection mode MTM lens based imaging system at MW frequencies. 
(a) Picture of uncovered letters

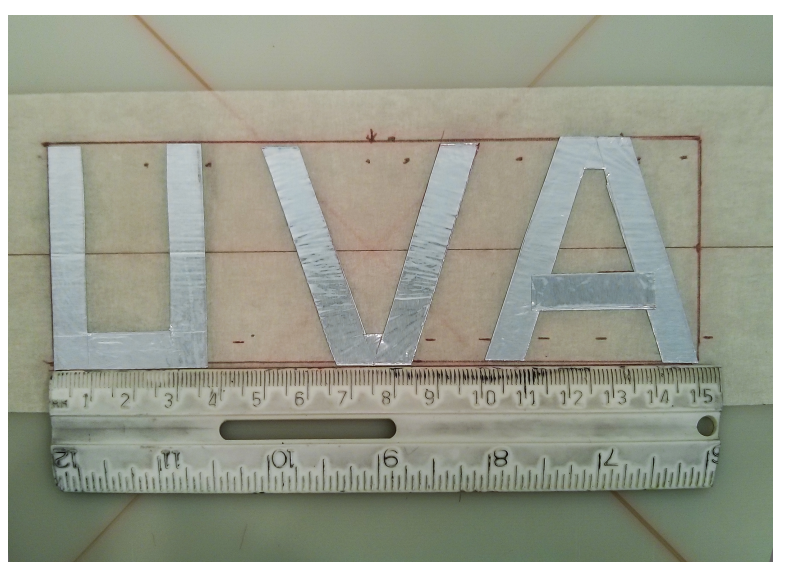

(b) 2-D MW image of hidden letters

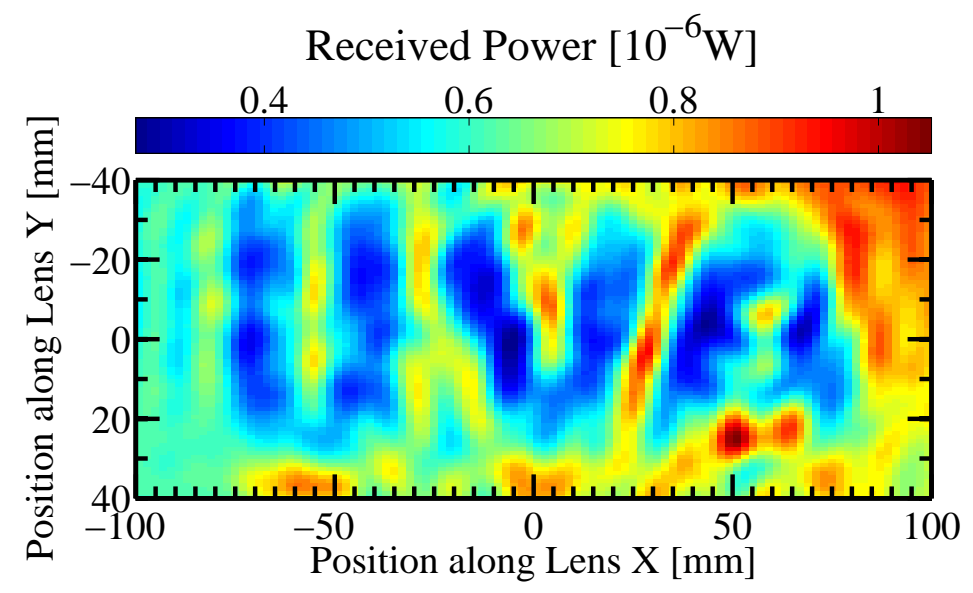

Figure 5.16: 2-D MW image of the hidden "UVA" logo taken with the 2-D HFL. The image shows the $\lambda / 2$ sized letter traces and "UVA" letters are clearly legible. 


\subsubsection{Effect of Polarization Rotation on Imaging}

As the MTM lens exhibited polarization rotation of the incident MW it was of interest to understand its effect on imaging. This was achieved by taking images with the receive antenna in co-polarized ( $0^{\circ}$ polarization angle, the antenna lead along the y-axis) and cross-polarized $\left(90^{\circ}\right.$ polarization angle, and antenna lead along the x-axis) conditions with respect to the source antenna $\left(0^{\circ}\right.$ polarization angle) and lens. For this study the dual holes and single holes were investigated. In an ordinary imaging configuration an isotropic lens would not be able to image into the cross-polarized condition. However, in the case of the asymmetric 2-D MTM lenses, the polarization rotation would manifest in the formation of the image [73]. In optics, the polarization can be used to greatly increase image contrast and can also be used to gain information on the object's index of refraction, stress, or birefringence. For this MTM based imaging system, capturing co- and cross-polarization signals allows for enhancing image contrast.

In fig. 5.17 it is shown that the normally co-polarized image does not carry all of the information contained in the image, as the polarization rotating nature of the HFL forced up to $22 \%$ of the information into the cross-polarized image. Fig. 5.17 shows the referenced image for the co-polarized detectors for the dual hole sample (fig. 5.17a) and the single hole sample (fig. 5.17b). The referenced images from the detector in the cross-polarized condition are shown for the dual hole sample in (fig. 5.17c) and the single hole sample in (fig. 5.17d). The co-polarized and cross-polarized images were referenced to their respective blank FR4 image data. Fig. 5.17e and fig. 5.17f show the compound image, where the information from both polarization states was added into a single image.

While not directly observable to the reader, the dynamic range of the image was increased. This enhancement in contrast can be valuable in imaging, as it makes objects more discernible from the background. In particular, the cross-polarized image could aid in lowering the number of false positives in a MW NDE inspection technique by either enhancing image contrast or revealing flaws that only respond to certain incident polarization states. The 
(a) Co-polarized image dual holes

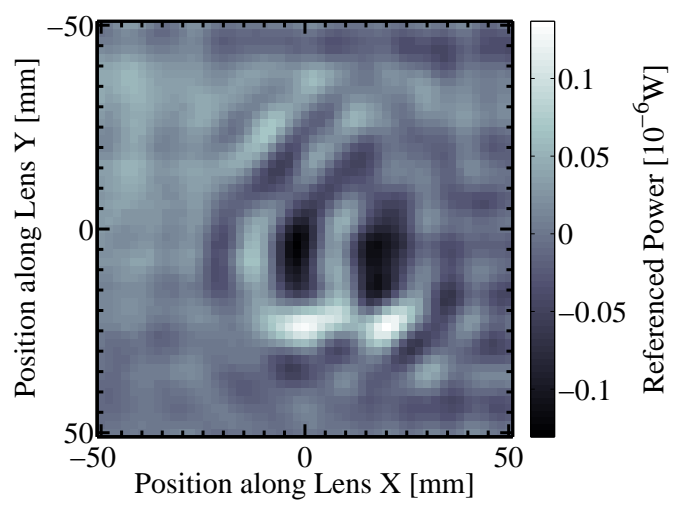

(c) Cross-polarized image dual holes

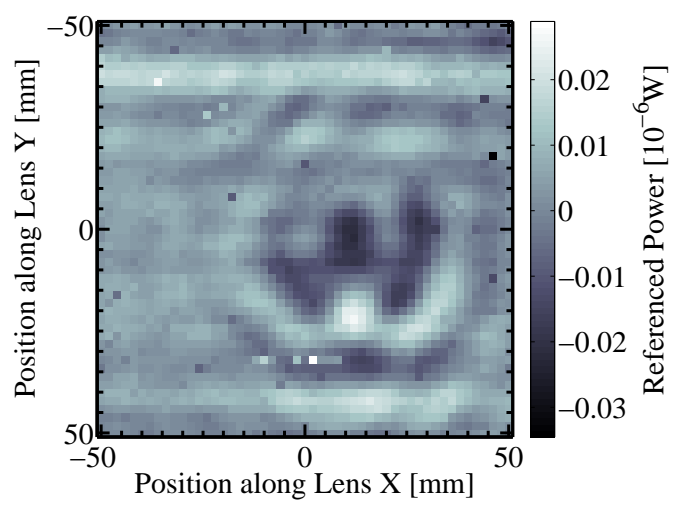

(e) Enhanced image dual holes

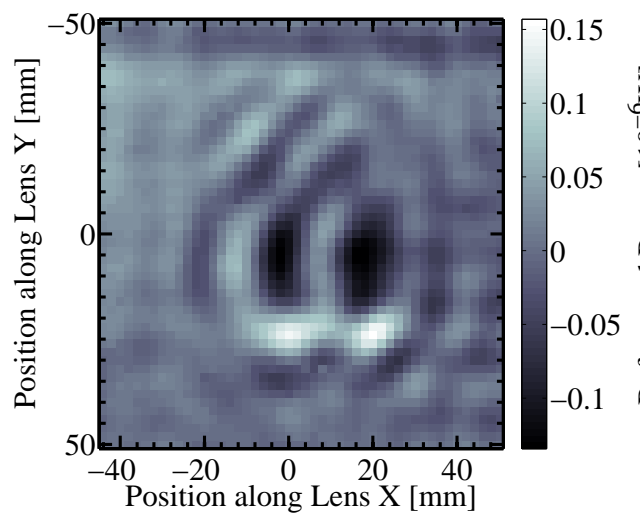

(b) Co-polarized image single hole

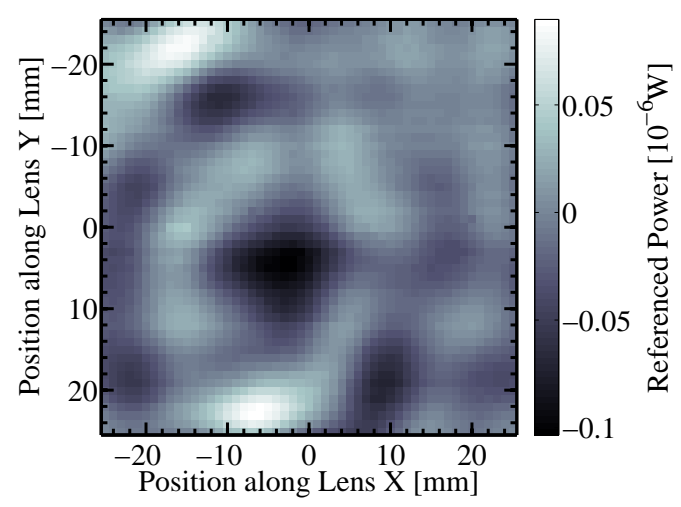

(d) Cross-polarized image single hole

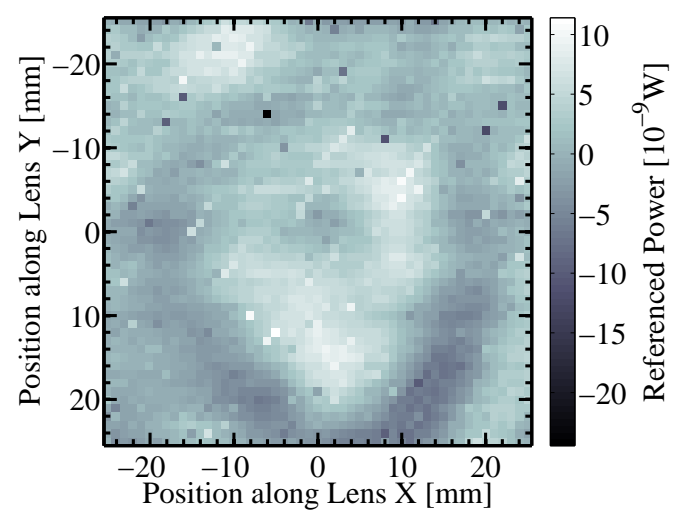

(f) Enhanced image single hole

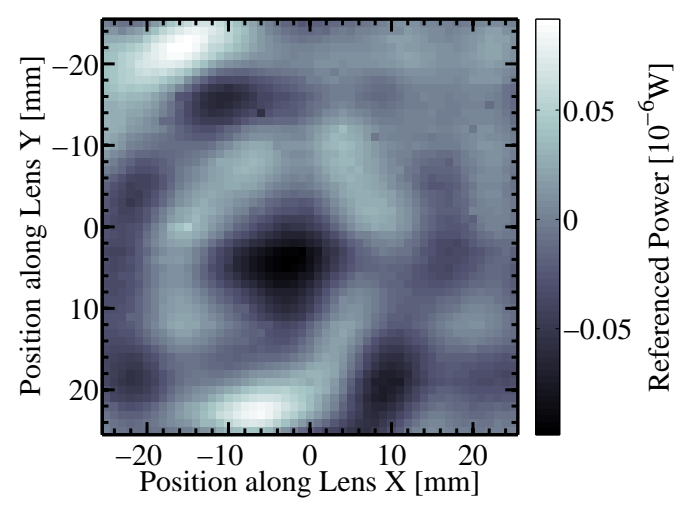

Figure 5.17: Polarization effect on imaging. Referenced co-polarized blind dual holes $(\lambda / 2$ feature size and $\lambda$ separation) and blind single hole $(\lambda / 3$ feature size) were compared to the images taken with the receive detector cross-polarized with respect to the source antenna. Adding the information from both polarization states into a single image increased the dynamic range and slightly enhanced the images. 
overall resolution and sensitivity was only slightly enhanced and neither the dual holes with $10 \mathrm{~mm}$ spacing or the smallest hole with $1.0 \mathrm{~mm}$ diameter was made distinguishable due to the polarization addition.

The study discussed in this section was the first of its kind in presenting 2-D images of samples with a reflection mode MTMs based imaging system. While the system proved to be limited in resolution to $0.66 \lambda$ and sensitivity to $0.12 \lambda$ sized objects, the MTM lens compares well to literature. The MTM HFL exceeded the stand off distance reported by Markley et al. [41], where measurements at $\lambda / 4$ were presented compared to the $4.05 \lambda$ lens to image distance of the 2-D HFL. Even though the resolution of the 2-D HFL does not beat the diffraction limit or come close to $0.259 \lambda$ of Markley et al., it is equivalent to the resolution $(\sim 0.64 \lambda)$ of a near-field Fresnel zone plate lens reported by Karimkashi et al. [40]. However, their focal distance would allow for much larger stand-off distance of 5.34 $\lambda$. It is important to point out that Karimkashi's results are based on FWHM data, and imaging of real objects was not carried out.

\subsection{Reflection Mode Imaging using Multi-Detectors}

A multi detector array was investigated for the purpose of improving upon single detector measurements by making use of detector summation and image sub-sampling. In the experiment setup, the sample was moved in reference to the the MTM lens imaging system. However, this is analogous to moving the system with respect to the sample. Therefore, the following discussion is referenced to moving the system with respect to the sample.

Detector summation made use of the five distributed detectors and summed their information into a single image. Sub-sampling was used to accelerate image acquisition; multiple detectors of the system interrogated the image plane at several locations and their information provided for an image. The multi-detector array was compared to single detector measurements in terms of resolution and sensitivity. The resolution study made use of the previously 
described blind dual holes and the sensitivity was evaluated with blind single hole samples. Fig. 5.18 and fig. 5.19, show the referenced power of the five detectors for the resolution study and sensitivity study. Each detector image was referenced to its respective measurement of a blank FR4. In both cases, the center detector contained more received signal as compared to the outside detectors. This was expected as the center detector was within the focus spot of the lens and the outside detectors were slightly outside of the focus. The detector information was subsequently analyzed for detector summation and image sub-sampling.

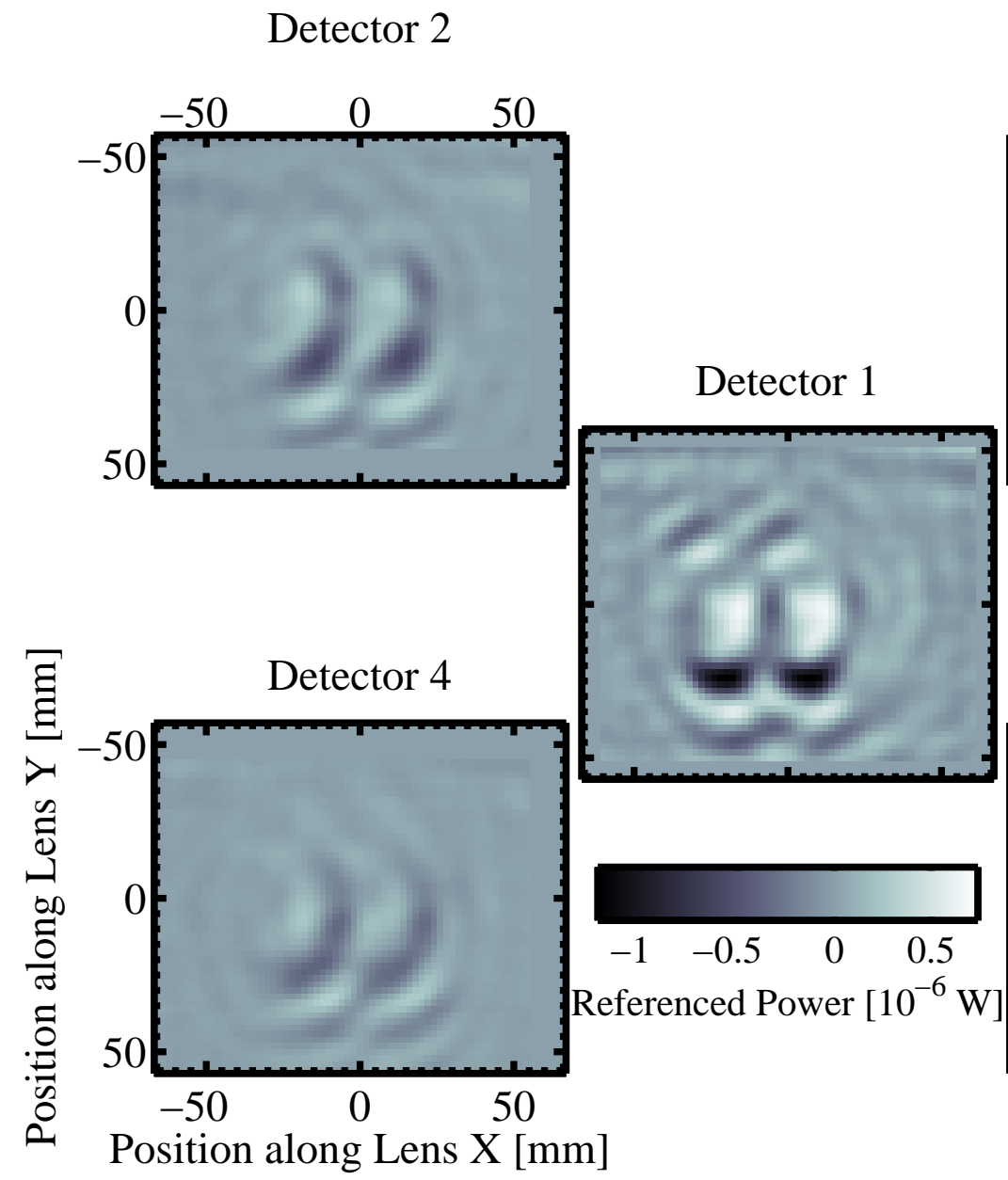

\section{Detector 3}

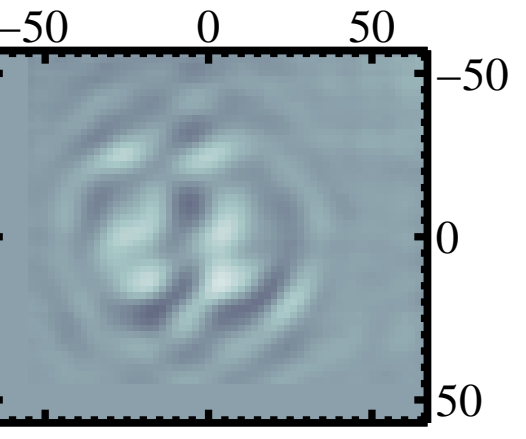

Detector 5

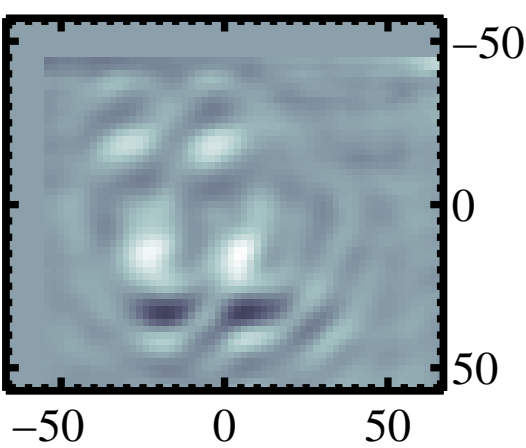

Figure 5.18: Multi-detector image of hidden dual holes. The dual hole $(\lambda / 2$ feature size, $1.5 \lambda$ separation) sample, images were referenced for each detector to a blank FR4 measurement. The figure shows the referenced measurements of each detector as the sample was swept in the $\mathrm{x}-\mathrm{y}$ plane. Detector summed images and sub-sampled images were retrieved from this referenced data. 


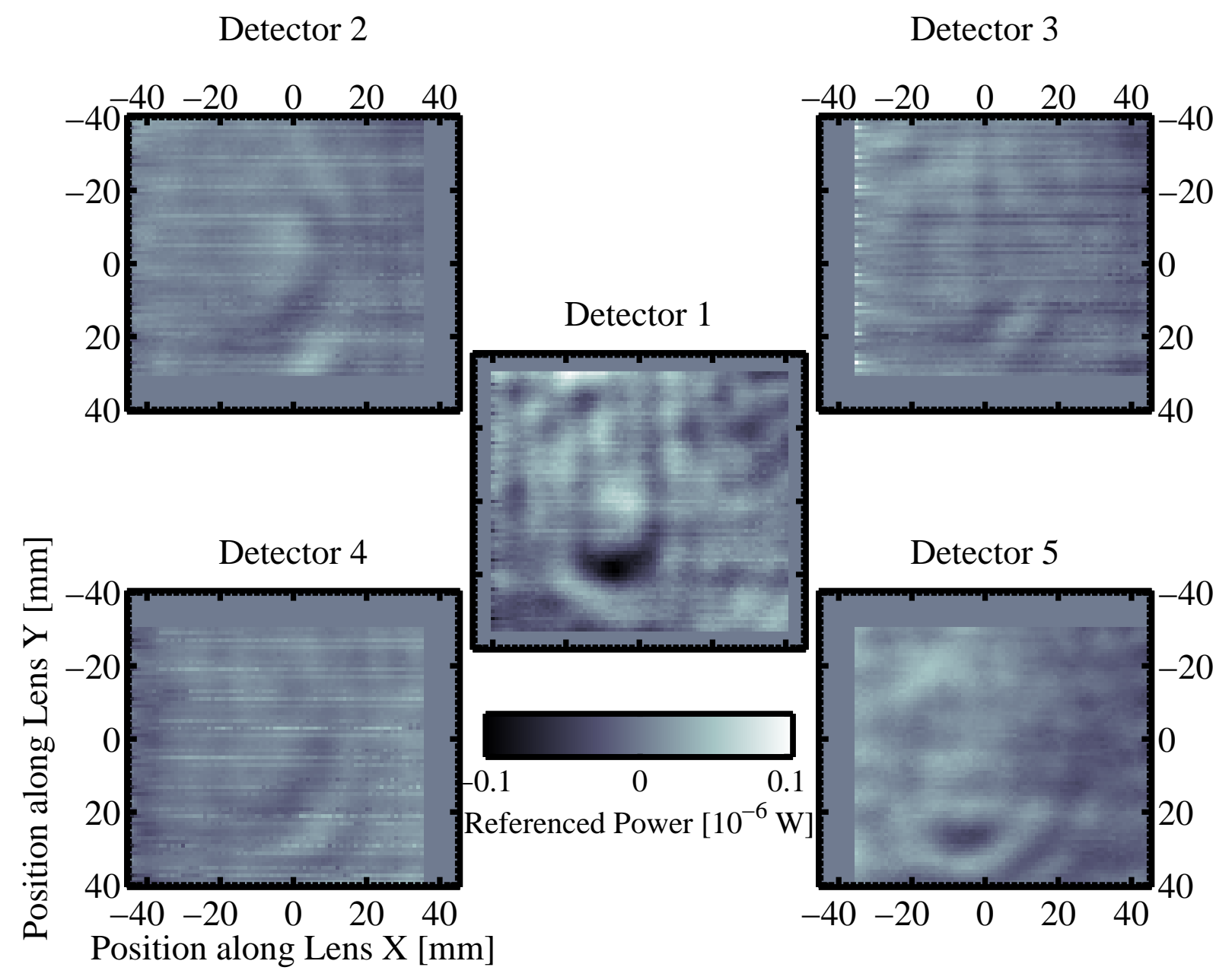

Figure 5.19: Multi-detector image of hidden single hole $(0.12 \lambda$ feature size $)$ sample. With the image referenced to the blank FR4 sample, the individual detectors were able to discern the presence of the subwavelength sized hole. This data was the basis for detector summation and sub-sampling.

In studying the resolution of the multi-detector array, the sub-sampling algorithm was as follows. The sub-sampling intervals were chosen such that each detector contributed similar amount of information to the image mosaic and coverage was at least $50 \%$ of the original image. The imaging system was stepped along the $\mathrm{x}$-axis and the $\mathrm{y}$-axis in $6 \mathrm{~mm}$ steps, as is shown in fig. 5.20a. The figure outlines the placement of the center detector of the array. Combining the information of all the detectors and their indexed position in the focal plane (see fig. 5.20b), it can be seen that the resultant image would cover the $\mathrm{x}$-y focal plane well. The sub-sampled image coverage was $55.7 \%$ of the original image. Furthermore, the 
(a) Single detector subsampling

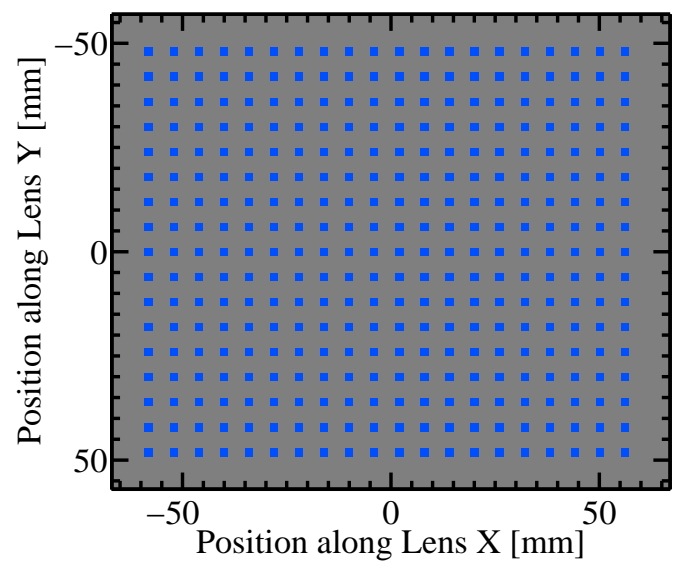

(b) Mosaic of multiple detectors

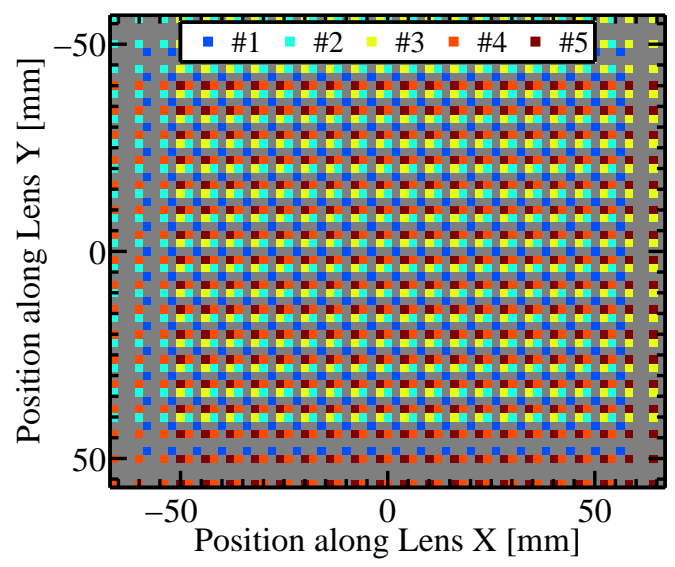

Figure 5.20: Multi-detector sub-sampling methodology for the blind dual hole $(\lambda / 2$ feature size, $1.5 \lambda$ separation) sample. (a) traces the interrogating positions of the center detector. (b) is the mosaic of all the detectors, where the figure shows the indexed position of the individual detectors interrogation positions in the focal plane.

detectors were well represented in the aggregate, where detector 1 through 5 made up 19.61\%, $20.59 \%, 19.6 \%, 20.59 \%$, and $19.61 \%$ of the final image mosaic. This sub-sampling technique reduced the number of steps to trace out the image, thus accelerated image acquisition. The multi-detector sub-sampling reduced image acquisition time by a factor of 5.1 for the dual hole sample. The factor was highly dependent on the geometric factors of the sub-sampling. In this configuration the focal plane was traced out with $20 \%$ fewer detector steps than compared to a single detector image with identical image coverage.

The large separation distance of the dual hole sample was well resolved for each of the imaging techniques. As was expected the summed detector data (see fig. 5.21a) increased the dynamic range as compared to a single detector image. The summed detector data showed a peak to peak distance of $28 \mathrm{~mm}$ and a trough of 0 , indicating that the multi-detector setup fully resolved the sample. The array data compared well to the single detector data. The sub-sampled data also matched the single detector data well (see fig. 5.21d). The multi detector array was also able to discern between dual holes of lesser separation (fig. 5.22), and was comparable in resolution to the reflection mode single detector imaging technique. 
(a) Sum of multiple detectors

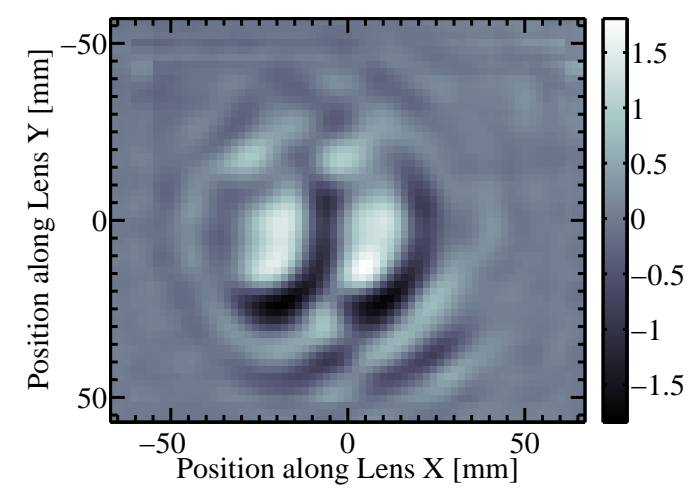

(c) Mosaic of multiple detectors

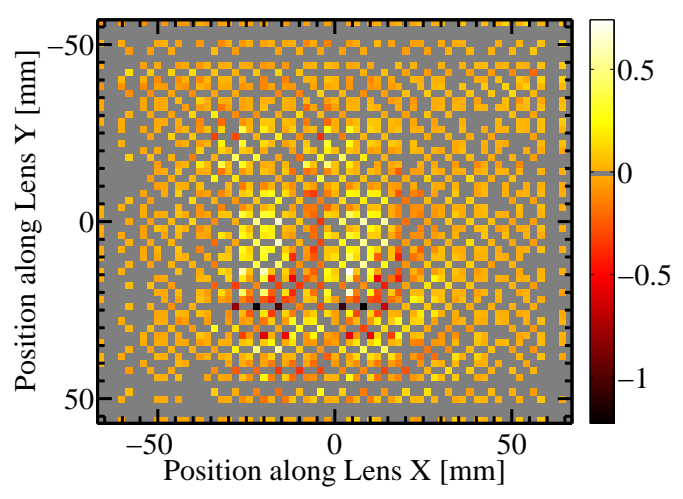

(b) Sub-sample center detector

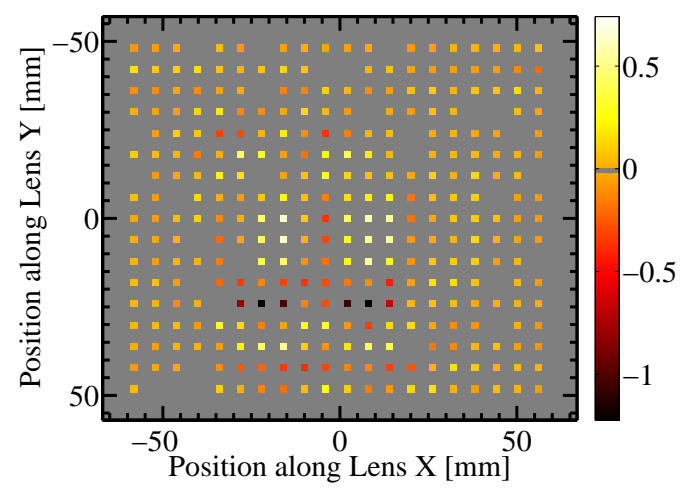

(d) Line Scan

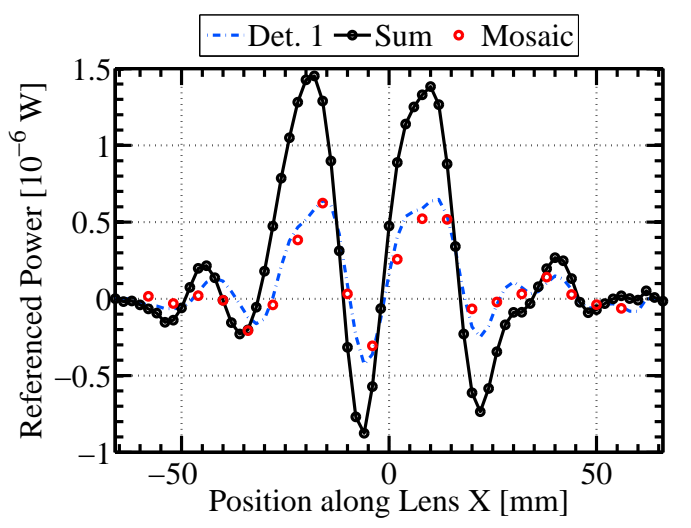

Figure 5.21: Multi-detector image captures of dual blind holes with $\lambda / 2$ feature size and $1.5 \lambda$ separation. (a) shows the summed detector data. This methodology increased the dynamic range of the image, but resolution was unaffected. (b) is the sub-sampled image using only the center detector of the multi detector array. (c) shows the image mosaic made up of all the detectors. $(\mathrm{d})$ the line scan along the $\mathrm{x}$-axis at $\mathrm{y}=0$ revealed that the dual holes were well resolved. 
(a) Hole separation $0.83 \lambda$

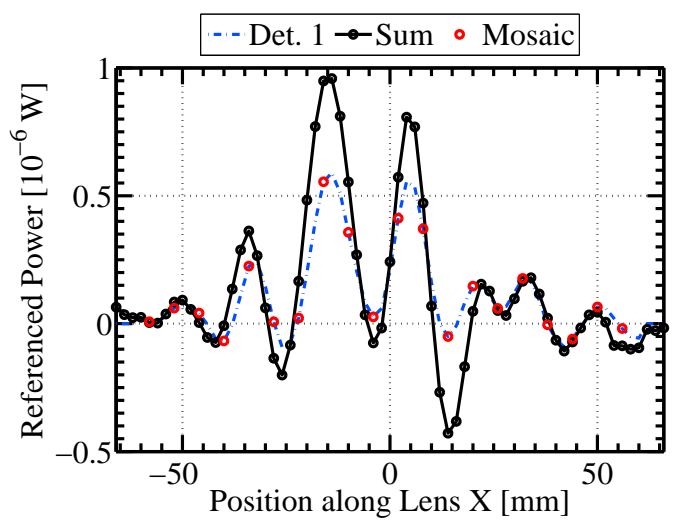

(b) Hole separation $0.66 \lambda$

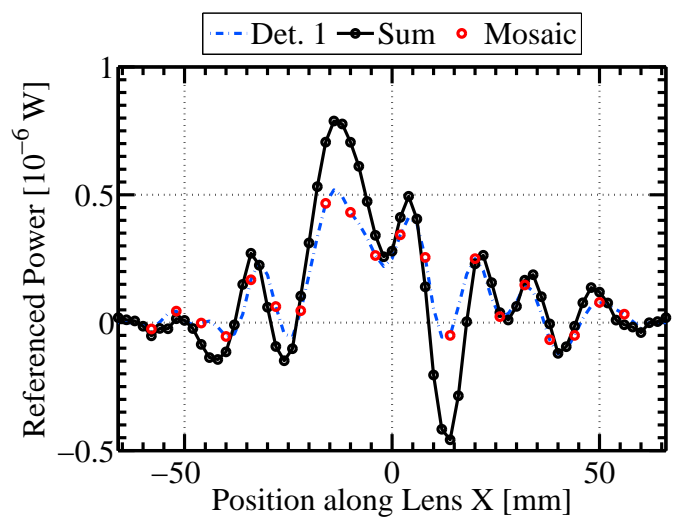

Figure 5.22: Multi-detector resolution performance is evaluated with the line scan of blind dual hole samples. The line scans compare the summed detector data to single detector data and the image mosaic. The summed detector data of (a) with a separation of $0.83 \lambda$ exhibited a peak to peak distance of $18 \mathrm{~mm}(\lambda)$ and a normalized power at the trough of 0.26 . The summed detector data in (b) with a separation of $0.66 \lambda$ exhibited a peak to peak distance of $18 \mathrm{~mm}(\lambda)$ and a normalized power at the trough of 0.56 . Both multi detector samples were comparable to single detector data found in table 5.2 .

Utilizing the multi detector array expanded the sensitivity of the system. While the single detector study was not able to immediately provide visual markers for distinguishing between the $0.12 \lambda$ size hole and the background, the multi detector study revealed the presence of the hole even before detector summation was applied (see fig. 5.19). As compared to the single detector data, the summed detector data clearly showed the presence of a single hole with $0.12 \lambda$ feature size.

Sub-sampling was performed for this study as well and the multi detector positions are outlined in fig. 5.23a and the individual detector representation is shown in the mosaic in fig. 5.23b. The image was sub-sampled along the $\mathrm{x}$ - and $\mathrm{y}$-axis in $2 \mathrm{~mm}$ increments for two intervals and then repeated after having been displaced by $6 \mathrm{~mm}$ (which was a different algorithm than the previous section). The resultant trace for the center detector (Detector 1) is shown in fig. 5.23a. This detector mosaic (fig. 5.23b), covered $50.3 \%$ of the original image, while accelerating image acquisition by a factor of 4.8. The individual detectors 1 through 5 were well represented in the image mosaic with a make up of $20.67 \%, 18.29 \%, 20.74 \%$, 
(a) Single detector subsampling

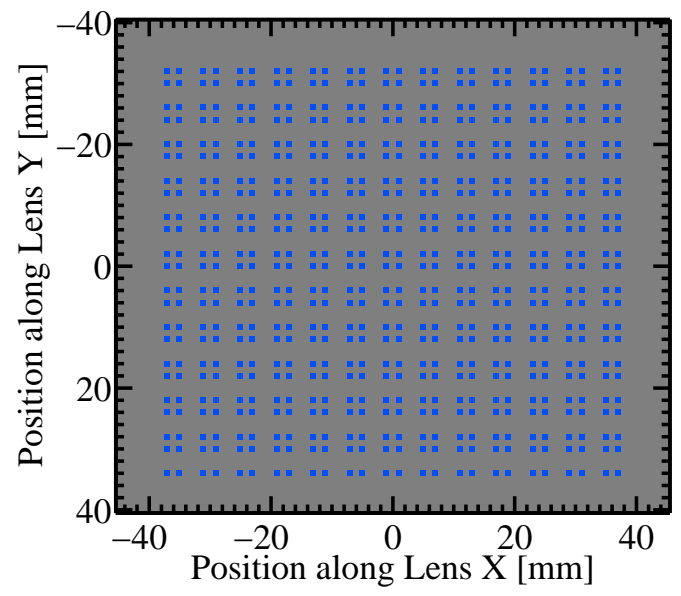

(b) Mosaic of multiple detectors

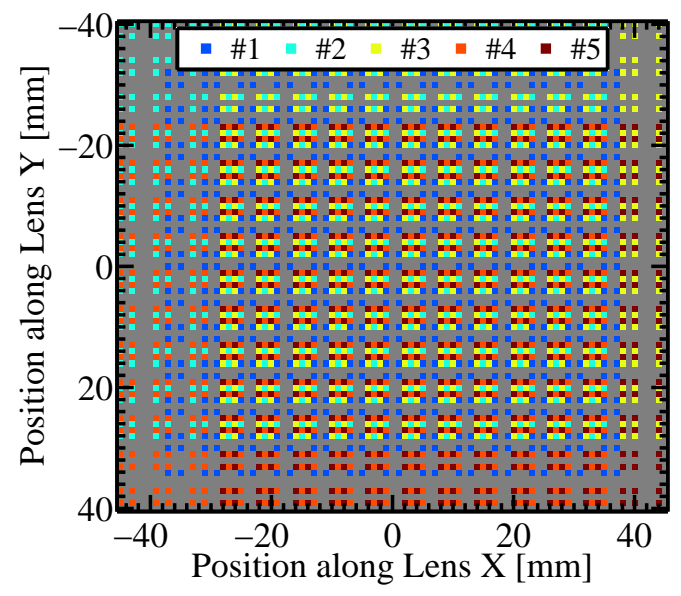

Figure 5.23: Multi-detector sub-sampling methodology for single holes. The array was stepped along the $\mathrm{x}$ - and $\mathrm{y}$-axis in $2 \mathrm{~mm}$ increments for two intervals and then displaced by $6 \mathrm{~mm}$. (a) the resultant trace for the center detector (Detector 1). (b) the resultant mosaic covered the focal plane well.

$21.29 \%$, and $19.01 \%$ respectively.

The single $0.12 \lambda$ sized hole was well detected using either single or summed detector measurements (see fig. 5.24). The mosaic data cuts down on the number of points along the $\mathrm{x}$-axis, however the feature was still observable.

The multi-detector array imaging technique improves upon single detector measurements in sensitivity. However the resolution of the system remains unchanged at $0.66 \lambda$ with a sample to image plane distance of $\sim 4 \lambda$, and a stand-off distance $f_{1}=26 \mathrm{~mm}$. 
(a) Sum of multiple detectors

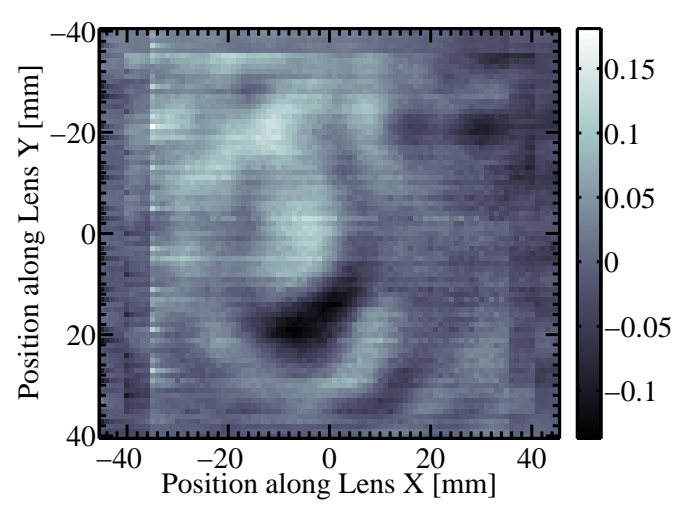

(c) Mosaic of multiple detectors

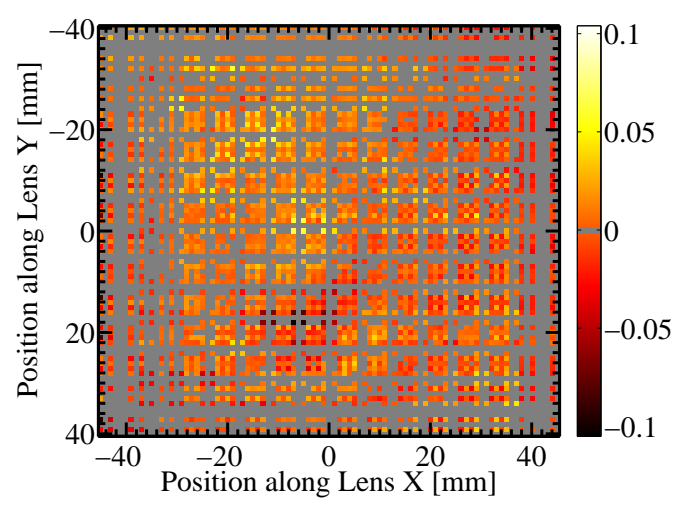

(b) Subsampled single detector

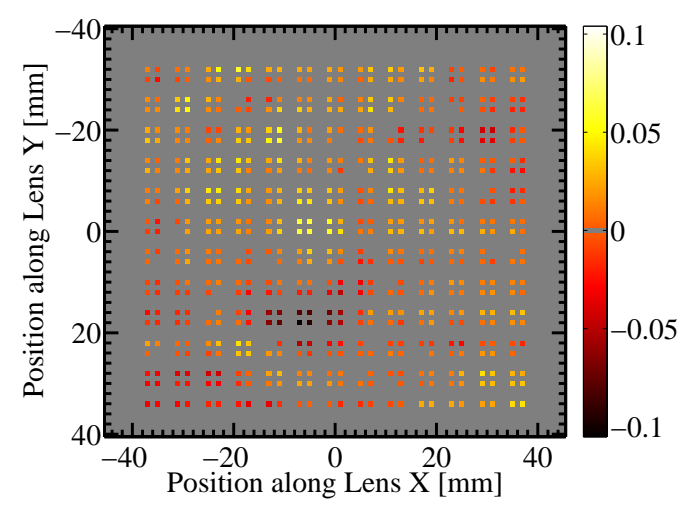

(d) Line Scan

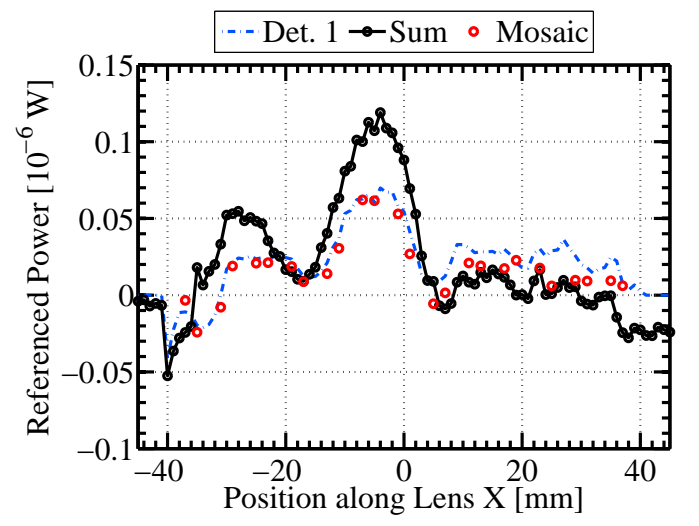

Figure 5.24: Multi-detector image captures of a blind hole with $0.12 \lambda$ feature size. (a) shows the summed detector data. The FWHM for the summed data was $16.5 \mathrm{~mm}(0.92 \lambda)$. As compared to single detector data, the dynamic range was increased and the feature was much more discernible from the background. (b) is the sub-sampled image using only the center detector of the multi detector array. (c) shows the image mosaic made up of all the detectors. (d) the line scan along the $\mathrm{x}$-axis at $\mathrm{y}=0$, revealed that the sub-wavelength sized hole was well detected. 


\subsection{Discussion}

Capable of both transmission mode and reflection mode imaging, the MTM lens based imaging system was investigated for its imaging characteristics such as resolution, sensitivity, depth of focus, and effect of polarization. The $3.63 \mathrm{GHz}$ resonant lens, in both 2-D and and 1-D configuration, was found to be unsuitable for imaging of samples due to its limitations in size and extinction coefficient, where as the $16.65 \mathrm{GHz}$ resonant lens was found to be a good candidate. The HFL was suitably large in width and height so as not to introduce edge effects in the imaging application and had a much lower extinction coefficient. The HFL's imaging characteristics were evaluated with several aperture and hole samples, with feature sizes on the order of the wavelength and or slightly below wavelength. For transmission mode imaging it was found that the HFL could resolve dual apertures with $\lambda / 2$ feature sizes and $\lambda$ separation, with a depth of focus of $15 \pm 1 \mathrm{~mm}(0.83 \pm 0.05 \lambda)$. The experiment matched modeling well. In reflection mode imaging the HFL was capable of resolving blind dual holes with $\lambda / 2$ feature sizes and $0.66 \lambda$ separation, and sensitive to features as small as $0.12 \lambda$, all while maintaining a stand-off distance of $1.44 \lambda$ and a sample to image plane distance of $\sim 4 \lambda$.

In examining the HFL for the effect of its polarization properties on imaging, it was found that a significant amount $(22 \%)$ of the signal and hence the image information was rotated into the cross-polarized condition. Adding the information of the co-polarized and crosspolarized image into a single image increased the dynamic range of the system accordingly. Alternatively, the cross-polarized image was also able to discern the subwavelength features, and could be used to analyze an image for false positive identification of hidden defects.

In addition, a multi-detector array was developed to surpass the single detector imaging technique. This detector array made use of detector summation to improve upon the dynamic range of the imaging system and sub-sampling to improve upon the imaging acquisition time. It was found that sub-sampling could improve upon image acquisition time up to 5.1 times, depending on the geometrical factors of the sub-sampling methodology. Furthermore, detector addition was useful in increasing the dynamic range of the image which resulted in 
a contrast enhancement. The multi-detector array did improve upon the sensitivity of the system, as the $0.12 \lambda$ feature size sample was distinguishable from the background. However, the resolution and stand-off distance remained the same.

The 2-D imaging of hidden subwavelength sized features, expanded upon previously established single point detection capabilities and the multi-detector array provided for a pathway to real time 2-D imaging. The limitations inherent to MTMs however has yet to be addressed. In a NDE application, MTM lenses would require usable stand-off distances $\left(f_{1}>\lambda\right)$, but the loss mechanisms inherent to SRR and RE MTM lenses prevent imaging beyond the diffraction limit.

In comparison to results from literature (see fig. 5.25), the HFL presented in this work matches well to modeled predictions on the resolution versus stand-off distance. In comparison to other lenses such as a near-field Fresnel lens, as discussed by Karimkashi et al. [40] the HFL has similar resolution, albeit much shorter stand-off distance. Furthermore, literature predicts possible near-field Fresnel lens designs that could exceed the performance of the HFL [77]. Other architectures, such as photonic crystals and thin MTM lenses exhibit subwavelength resolution, however present challenging stand-off distance requirements such that a near-field probe outperforms them $[12,16,41,78]$. It is of note that in order for the HFL to surpass Abbe's diffraction limit, equation 2.22 predicts that the HFL would require an extinction coefficient that is four orders of magnitude smaller (see fig. 5.26). Therefore, MTM lenses would require much further research into minimizing absorption losses due to resonant characteristics, polarization losses, and material parameters. For MW imaging at subwavelength resolution, the HFL data presented in this work shows that it is a good candidate. It provides a much greater stand-off distance and better resolution than proven near-field imaging systems realized to-date. 


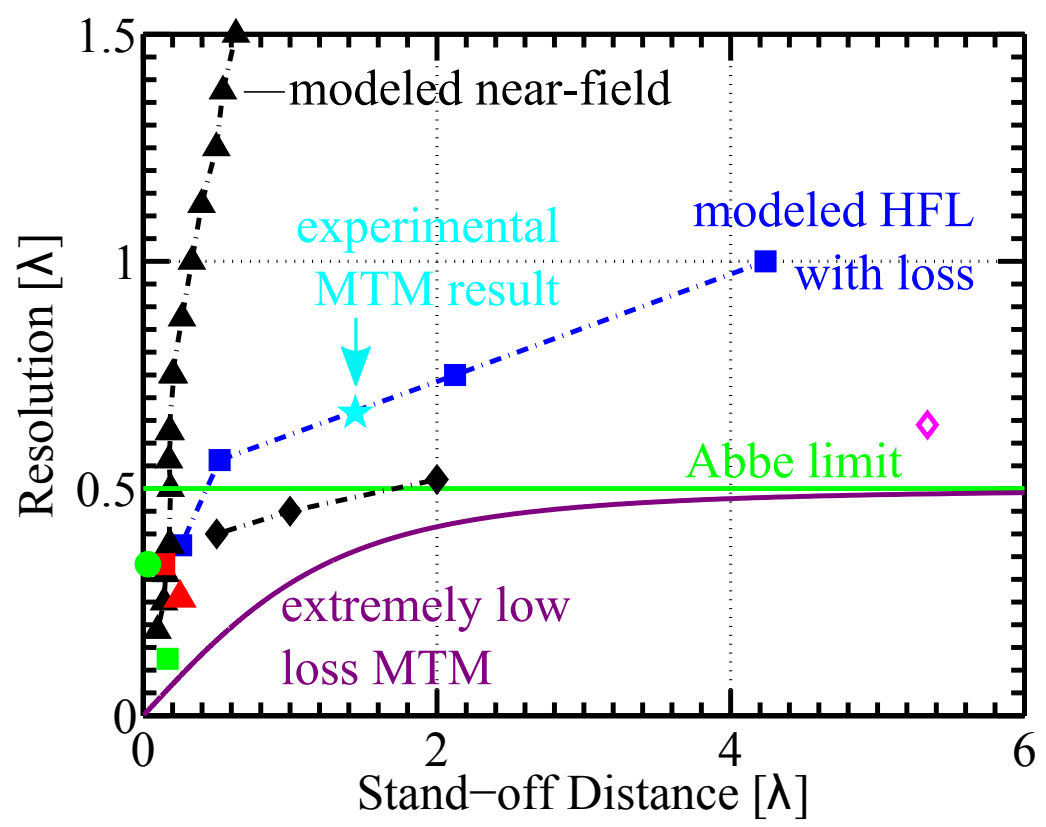

Figure 5.25: Resolution versus stand-off distance for various imaging systems. This figure compares results presented in this dissertation with results from literature.

$\checkmark$ Modeled near-field Fresnel lens [77]; $\diamond$ Experimental near-field Fresnel lens [40];

-Photonic crystal [12]; - SRR and RE MTM lens [16];

- MTM lens [78]; $\Delta$ Near-field probe [41];

Figure reproduced with permission of author [71].

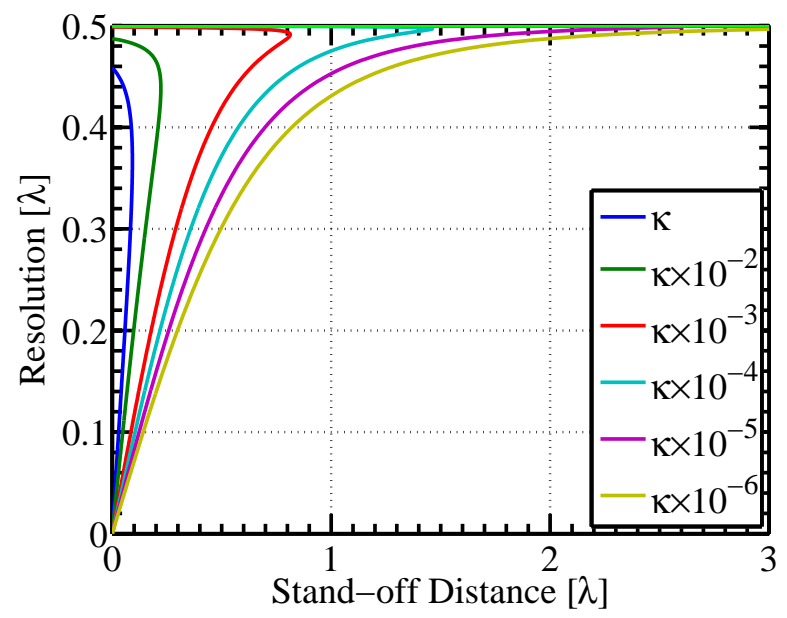

Figure 5.26: The effect of absorption on resolution versus stand-off distance is shown in this figure. The curves were generated using equation 2.22 with the HFL's $\kappa=0.10$. In order to beat the diffraction limit at the HFL's stand-off distance of $f_{1}=1.44 \lambda$ the lens would require an extinction coefficient of $\kappa * 10^{-4}$. Figure reproduced with permission of author [71]. 


\section{Chapter 6: Conclusions and Future}

\section{Work}

MTMs research is a relatively new field that has exhibited tremendous growth since its first successful demonstration in 2000. While advances have been made in developing MTMs throughout the electromagnetic spectrum, applications in sensor systems and devices are just beginning to follow. Of particular interest is the microwave MTM lens, which is suited to imaging applications because its long wavelengths give the ability to peer inside of materials, while subwavelength resolution will render small details. This study expands upon the current state of MTM lens based imaging and highlights the capabilities and limitations of a practical system.

The imaging system presented in this dissertation was the first of its kind to use a MW MTM lens in reflection mode to provide for 2-D images of hidden objects. In analyzing the EM properties of the MTM lenses it was found that they were highly sensitive to the incident polarization, leading to unwanted rotation of the polarization state of transmitted MWs. The effect of the polarization rotation in MTM lenses on image performance was analyzed, and is the first such discussion in literature.

Furthermore, the current state of MTM based imaging systems has been expanded upon by using a multi-detector array. This decreased image acquisition time while giving the potential of increasing the contrast of the image. The overall system provided for similar image quality as a near-field measurement at much greater stand-off distance than the near-field.

In studying the polarization properties of the MTM lenses, the coupling mechanisms between the MTM constituent elements and incident MWs were analyzed. This work was able to link the cause for the polarization rotation to the asymmetric unit cell arrangement of the SRR constituent elements. Imaging performance was affected by this asymmetric 
unit cell arrangement and resulted in forming up to $22 \%$ of the image information in the cross-polarized condition. In ordinary imaging experiments, where only the co-polarized state would be considered, this information would be lost and image quality would be affected. More importantly, the mechanism responsible for this polarization rotation caused a reduction in transmission of the negative index of refraction resonance, leading to higher absorption losses and decreased resolution. Modeling showed that for the 2-D LFL, a symmetric unit cell would raise the transmission at resonance to $-22.64 \mathrm{~dB}$ as compared to $-25.68 \mathrm{~dB}$ found in the asymmetric unit cell LFL, and would thus increase the resolution.

In imaging system, knowledge of the polarization rotation could be used to orient the detector to obtain the maximum signal and contrast of the image. Conversely, the polarization properties of the MTM lens could be leveraged for NDE applications, where interrogating an object with circular polarization would be highly desirable. Polarization dependent reflection coefficients could be used to analyze an object for its birefringence, material properties, as well as environmental factors such as stress.

The use of a multi-detector array was crucial in speeding up image acquisition by a factor of 5 as well as increase the dynamic range of the image. Furthermore, the multi-detector array could be arranged in such a way as to simultaneously interrogate the co- and cross-polarized image plane in order to improve image acquisition time as well as enhance image contrast. A redesign of the MTM lens to a symmetric unit cell arrangement would also eliminate the tilt in the image, allowing for monostatic source and detector placement. In moving forward with the multi-detector array, the diode detectors could be switched for heterodyne detectors, which would allow for increased contrast through phase information in the image.

There are several design challenges that have to be overcome in order for MTMs to become practical in imaging applications. While MTM flat lenses allow for very compact lens designs, they are inherently limited by their thickness. Thin $(<\lambda)$ lenses are subject to short focal lengths, however have the ability to surpass the diffraction limit. Thick $(\geq \lambda)$ lenses suffer from absorption that directly limits their resolution capabilities. While the HFL 
demonstrated a subwavelength resolution of $0.66 \lambda$ and sensitivity of $0.12 \lambda$ at a stand-off distance of $1.44 \lambda$ and object to image plane length of $4 \lambda$, the absorption of the lens has to be lowered in order for the HFL to surpass the Abbe diffraction limit of $0.5 \lambda$.

The overall MTM lens system was also compared to near-field imaging and a reported near-field Fresnel zone plate lens. It was found that the MTM system was not able to surpass the diffraction limit, however it provided for similar image quality as the near-field image from a probe at a much greater stand-off distance $(1.44 \lambda)$ as compared to the near-field's $(0.17 \lambda)$. Compared to a near-field Fresnel zone plate lens, the MTM system had similar resolution, albeit much shorter stand-off distance [40]. The near-field Fresnel zone plate lens from Karimkashi et al. [40] has yet to be demonstrated in a near-field MW imaging application. Furthermore, modeling predicts Fresnel lens designs that could have better resolution and stand-off distance than the HFL [77].

Further optimization of constituent elements and unit cell arrangements could lead to MTM designs with imaginary index of refraction on the order of 0.01 [79]. Other considerations, such as using lower loss and thinner dielectrics, could also improve upon the loss mechanisms inherent to the lens. Primarily, however; the SRR and RE design approach for MTMs has been surpassed by less lossy designs such as a double S-shaped resonator [80]. These types of resonators offer a distinct advantage by combining the SRR and RE elements into a single structure. Furthermore, Chen et al. promise an increase in bandwidth [81], as well as a loss reduction from $-6.53 \mathrm{~dB} / \mathrm{cm}$ for $\mathrm{SRR}$ and RE MTMs to $-1.75 \mathrm{~dB} / \mathrm{cm}$ for S-shaped MTMs [80]. In comparison, the HFL has a loss of $-2.90 \mathrm{~dB} / \mathrm{cm}$; however, Chen et al. use FR4 substrate [80] whereas the HFL uses low loss Rogers Duroid dielectric. Building a MTM lens based on S-shaped MTMs on a Rogers Duroid substrate could lead to a state of the art low loss MTM lens. Other MTM structures such as "Fishnet" [82-84] and chiral $[70,85]$ MTM structures should also be investigated for their performance. Therefore, by changing the design parameters for the MTM, a low loss MTM lens could be achieved, making flat lenses a good candidate for non-diffraction limited imaging at practical stand-off distances. 
Additionally, by increasing the stand-off distances of these MTM lenses could allow for 3-D imaging in dielectric media, leading to a host of NDE applications in biomedicine, quality control, infrastructure and security industries.

MTM lenses are not solely limited to flat lenses. As the constituent elements allow for any type of index of refraction (both positive and negative), novel lens designs can be achieved. While these lenses would not be able to enhance evanescent waves, they would be useful in designing complex lens elements, such as gradient index lenses [86-88]. The resonant characteristics of the SRRs themselves are just beginning to be explored in wireless sensing applications. Recent literature has reported on tunable MTM resonators [89], novel THz sensors which use SRRs as pixels at very high density [90], and SRR based sensors for detecting the presence of bacteria [91-93]. 


\section{List of Publications and Presentations}

1. D. A. McGillivray, R. L. Cravey, K. L. Dudley, and M. C. Gupta, "Metamaterial Lens based 2-D Microwave Imaging System", IEEE Transactions on Antennas and Propagation (submitted).

2. D. A. McGillivray, R. L. Cravey, K. L. Dudley, E. Vedeler, and M. C. Gupta, "Polarization Properties of 2-D Split Ring Resonator and Rod Type Metamaterial Lens", Microwave and Optical Technology Letters, 56(9):2146-2150, September 2014.

3. D. A. McGillivray, R. L. Cravey, K. L. Dudley, E. Vedeler, and M. C. Gupta, "Polarization Properties of a One Dimensional Metamaterial Lens", Microwave and Optical Technology Letters, 56(5):1218-1222, May 2014.

4. D. A. McGillivray, R. L. Cravey, K. L. Dudley, E. Vedeler, and M. C. Gupta, "Polarization properties of 2D and 1D metamaterial lenses", presented at: SPIE Optics \& Photonics - Metamaterials: Fundamentals and Applications VI conference, San Diego, CA 25-29 August 2013. 


\section{Bibliography}

[1] H. Fountain, "Strides in materials, but no invisibility cloak," The New York Times, p. D4, November 2010. [Online]. Available: http://www.nytimes.com/2010/11/09/science/09meta.html

[2] D. Schurig, J. J. Mock, B. J. Justice, S. A. Cummer, J. B. Pendry, A. F. Starr, and D. R. Smith, "Metamaterial electromagnetic cloak at microwave frequencies," Science, vol. 314, no. 5801, pp. 977-980, November 2006.

[3] D. R. Smith and N. Kroll, "Negative refractive index in left-handed materials," Physical Review Letters, vol. 85 , no. 14 , pp. 2933-2936, October 2000.

[4] J. B. Pendry, "Negative refraction makes a perfect lens," Physical Review Letters, vol. 85, no. 18, pp. 3966-3969, October 2000.

[5] Z. Liu, H. Lee, Y. Xiong, C. Sun, and X. Zhang, "Far-field optical hyperlens magnifying sub-diffractionlimited objects," Science, vol. 315, no. 5819, p. 1686, March 2007.

[6] D. R. Smith, W. J. Padilla, D. C. Vier, S. C. Nemat-Nasser, and S. Schultz, "Composite medium with simultaneously negative permeability and permittivity," Physical Review Letters, vol. 84, no. 18, pp. 4184-4187, May 2000.

[7] J. B. Pendry, A. J. Holden, D. J. Robbins, and W. J. Stewart, "Magnetism from conductors and enhanced nonlinear phenomena," Microwave Theory and Techniques, IEEE Transactions on, vol. 47, no. 11, pp. 2075-2084, November 1999.

[8] N. Katsarakis, T. Koschny, M. Kafesaki, E. N. Economou, and C. M. Soukoulis, "Electric coupling to the magnetic resonance of split ring resonators," Applied Physics Letters, vol. 84, no. 15, pp. 2943-2945, April 2004.

[9] J. B. Pendry, A. J. Holden, W. J. Stewart, and I. Youngs, "Extremely low frequency plasmons in metallic mesostructures," Physical Review Letters, vol. 76, no. 25, pp. 4773-4776, June 1996.

[10] V. G. Veselago, "The electrodynamics of substances with simultaneously negative values of $\epsilon$ and $\mu$," Physics-Uspekhi, vol. 10, no. 4, pp. 509-514, January 1968.

[11] Z. Liu, N. Fang, T. Yen, and X. Zhang, "Rapid growth of evanescent wave by a silver superlens," Applied Physics Letters, vol. 83, no. 25, pp. 5184-5186, December 2003.

[12] E. Cubukcu, K. Aydin, E. Ozbay, S. Foteinopoulou, and C. M. Soukoulis, "Subwavelength resolution in a two-dimensional photonic-crystal-based superlens," Physical Review Letters, vol. 91, no. 20, pp. $207401.1-4$, November 2003.

[13] K. Aydin, K. Guven, C. M. Soukoulis, and E. Ozbay, "Observation of negative refraction and negative phase velocity in left-handed metamaterials," Applied Physics Letters, vol. 86, no. 12, pp. $124102.1-3$, March 2005.

[14] A. Grbic and G. V. Eleftheriades, "Negative refraction, growing evanescent waves, and sub-diffraction imaging in loaded transmission-line metamaterials," Microwave Theory and Techniques, IEEE Transactions on, vol. 51, no. 12, pp. 2297-2305, January 2003. 
[15] X. Zhang and Z. Liu, "Superlenses to overcome the diffraction limit," Nature Materials, vol. 7, no. 6, pp. 435-441, June 2008.

[16] K. Aydin, I. Bulu, and E. Ozbay, "Subwavelength resolution with a negative-index metamaterial superlens," Applied Physics Letters, vol. 90, no. 25, pp. 254 102.1-3, June 2007.

[17] A. N. Lagarkov and V. N. Kissel, "Near-perfect imaging in a focusing system based on a left-handedmaterial plate," Physical Review Letters, vol. 92, no. 7, pp. 077 401.1-4, February 2004.

[18] J. S. Lih, Y. S. Wang, M. C. Lu, Y. C. Huang, K. H. Chen, J. L. Chern, and L. E. Li, "Experimental realization of breaking diffraction limit by planar negative-index metamaterials in free space," Europhysics Letters, vol. 69, no. 4, pp. 544-548, February 2005.

[19] D. M. Sheen, D. L. McMakin, and T. E. Hall, "Three-dimensional millimeter-wave imaging for concealed weapon detection," Microwave Theory and Techniques, IEEE Transactions on, vol. 49, no. 9, pp. 1581-1592, September 2001.

[20] E. C. Fear, X. Li, S. C. Hagness, and M. A. Stuchly, "Confocal microwave imaging for breast cancer detection: Localization of tumors in three dimensions," Biomedical Engineering, IEEE Transactions on, vol. 49, no. 8, pp. 812-822, August 2002.

[21] J. E. Bridges, S. C. Hagness, A. Sahakian, and A. Taflov, "Microwave antenna for cancer detection system," US Patent 6,061,589, May 9, 2000.

[22] S. Kharkovsky and R. Zoughi, "Microwave and millimeter wave nondestructive testing and evaluationoverview and recent advances," Instrumentation $\mathscr{E}$ Measurement Magazine, IEEE, vol. 10, no. 2, pp. 26-38, 2007.

[23] M. Q. Feng, F. De Flaviis, and Y. J. Kim, "Use of microwaves for damage detection of fiber reinforced polymer-wrapped concrete structures," Journal of Engineering Mechanics, vol. 128, no. 2, pp. 172-183, February 2002.

[24] A. J. Witten, J. E. Molyneux, and J. E. Nyquist, "Ground penetrating radar tomography: Algorithms and case studies," Geoscience and Remote Sensing, IEEE Transactions on, vol. 32, no. 2, pp. 461-467, March 1994.

[25] B. Panzner, A. Joestingmeier, and A. Omar, "K a-band dielectric lens antenna for resolution enhancement of a gpr," in Antennas, Propagation and EM Theory, 2008. ISAPE 2008. 8th International Symposium on. Kunming, China: IEEE, November 2008, pp. 31-34.

[26] Y. Deng and X. Liu, "Electromagnetic imaging methods for nondestructive evaluation applications," Sensors, vol. 11, no. 12, pp. 11774-11808, December 2011.

[27] N. Ghavami, G. Tiberi, D. J. Edwards, and A. Monorchio, "Uwb microwave imaging of objects with canonical shape," Antennas and Propagation, IEEE Transactions on, vol. 60, no. 1, pp. 231-239, January 2012.

[28] C. Stephanis and G. Hampsas, "Imaging with microwave lens," Antennas and Propagation, IEEE Transactions on, vol. 28, no. 1, pp. 49-52, January 1980.

[29] A. I. Carswell and C. Richard, "Focal properties of microwave lenses with small f-numbers," Applied Optics, vol. 10, no. 2, pp. 346-357, February 1971.

[30] A. Petosa and A. Ittipiboon, "Design and performance of a perforated dielectric fresnel lens," IEE Proceedings-Microwaves, Antennas and Propagation, vol. 150, no. 5, pp. 309-314, October 2003.

[31] O. Quevedo-Teruel, W. Tang, R. C. Mitchell-Thomas, A. Dyke, H. Dyke, L. Zhang, S. Haq, and Y. Hao, "Transformation optics for antennas: why limit the bandwidth with metamaterials?" Scientific Reports, vol. 3, pp. 1903.1-5, May 2013.

[32] R. Yang, W. Tang, and Y. Hao, "A broadband zone plate lens from transformation optics," Optics Express, vol. 19, no. 13, pp. 12 348-12 355, 2011. 
[33] R. K. Raney, "Synthetic aperture imaging radar and moving targets," Aerospace and Electronic Systems, IEEE Transactions on, vol. AES-7, no. 3, pp. 499-505, May 1971.

[34] M. Cheney and B. Borden, "Problems in synthetic-aperture radar imaging," Inverse Problems, vol. 25, no. 12, pp. 123 005.1-18, December 2009.

[35] C. P. Vlahacos, R. C. Black, S. M. Anlage, A. Amar, and F. C. Wellstood, "Near-field scanning microwave microscope with $100 \mu \mathrm{m}$ resolution," Applied Physics Letters, vol. 69, no. 21, pp. 3272-3274, September 1996.

[36] X.-D. Xiang and C. Gao, "Quantitative complex electrical impedance microscopy by scanning evanescent microwave microscope," Materials Characterization, vol. 48, no. 2, pp. 117-125, April 2002.

[37] S. M. Anlage, V. V. Talanov, and A. R. Schwartz, "Principles of near-field microwave microscopy," in Scanning Probe Microscopy, S. Kalinin and A. Gruverman, Eds. Springer New York, 2007, pp. 215-253.

[38] M. Ravan, R. K. Amineh, and N. K. Nikolova, "Two-dimensional near-field microwave holography," Inverse Problems, vol. 26, no. 5, pp. 055 011.1-25, May 2010.

[39] L. Markley and G. V. Eleftheriades, "A near-field probe for subwavelength-focused imaging," Microwave Theory and Techniques, IEEE Transactions on, vol. 58, no. 3, pp. 551-558, May 2010.

[40] S. Karimkashi and A. A. Kishk, "Focusing properties of fresnel zone plate lens antennas in the near-field region," Antennas and Propagation, IEEE Transactions on, vol. 59, no. 5, pp. 1481-1487, May 2011.

[41] L. Markley and G. V. Eleftheriades, "Two-dimensional subwavelength-focused imaging using a near-field probe at a $\lambda / 4$ working distance," Journal of Applied Physics, vol. 107, no. 9, pp. 093 102.1-5, May 2010.

[42] D. Shreiber, M. C. Gupta, and R. L. Cravey, "Microwave nondestructive evaluation of dielectric materials with a metamaterial lens," Sensors and Actuators A: Physical, vol. 144, no. 1, pp. 48-55, May 2008.

[43] D. Shreiber, M. C. Gupta, and R. L. Cravey, "Comparative study of 1-d and 2-d metamaterial lens for microwave nondestructive evaluation of dielectric materials," Sensors and Actuators A: Physical, vol. 165, no. 2, pp. 256-260, February 2011.

[44] X.-X. Liu and A. Alu, "Limitations and potentials of metamaterial lenses," Journal of Nanophotonics, vol. 5, no. 1, pp. 053 509.1-13, June 2011.

[45] D. R. Smith, D. Schurig, M. Rosenbluth, S. Schultz, S. A. Ramakrishna, and J. B. Pendry, "Limitations on subdiffraction imaging with a negative refractive index slab," Applied Physics Letters, vol. 82, no. 10, pp. 1506-1508, March 2003.

[46] V. A. Podolskiy and E. E. Narimanov, "Near-sighted superlens," Optics Letters, vol. 30, no. 1, pp. 75-77, January 2005.

[47] X. Chen, T. M. Grzegorczyk, B. Wu, J. Pacheco Jr, and J. A. Kong, "Robust method to retrieve the constitutive effective parameters of metamaterials," Physical Review E, vol. 70, no. 1, pp. 016608.1-7, July 2004.

[48] L. Chen, Z. Lei, R. Yang, X. Shi, and J. Zhang, "Determining the effective electromagnetic parameters of bianisotropic metamaterials with periodic structures," Progress In Electromagnetics Research M, vol. 29, pp. 79-93, February 2013.

[49] Z. Szabo, G. Park, R. Hedge, and E. Li, "A unique extraction of metamaterial parameters based on kramers-kronig relationship," Microwave Theory and Techniques, IEEE Transactions on, vol. 58, no. 10, pp. 2646-2653, October 2010.

[50] A. Grbic and G. V. Eleftheriades, "Practical limitations of subwavelength resolution using negativerefractive-index transmission-line lenses," Antennas and Propagation, IEEE Transactions on, vol. 53, no. 10, pp. 3201-3209, October 2005. 
[51] H. Cory, Y. J. Lee, Y. Hao, and C. G. Parini, "Use of conjugate dielectric and metamaterial slabs as radomes," IET Microwaves, Antennas 83 Propagation, vol. 1, no. 1, pp. 137-143, February 2007.

[52] A. Alù and N. Engheta, "Pairing an epsilon-negative slab with a mu-negative slab: resonance, tunneling and transparency," Antennas and Propagation, IEEE Transactions on, vol. 51, no. 10, pp. 2558-2571, October 2003.

[53] H. Cory and C. Zach, "Wave propagation in metamaterial multi-layered structures," Microwave and Optical Technology Letters, vol. 40, no. 6, pp. 460-465, March 2004.

[54] G. Wang, Y. Gong, and H. Wang, "On the size of left-handed material lens for near-field target detection by focus scanning," Progress In Electromagnetics Research, vol. 87, pp. 345-361, October 2008.

[55] G. Wang, J. R. Fang, and X. T. Dong, "Refocusing of backscattered microwaves in target detection by using lhm flat lens," Optics Express, vol. 15, no. 6, pp. 3312-3317, March 2007.

[56] M. Meyer and L. Herbeck, "Microwave effects on cfrp processing," in SAMPE Europe Conference and Exhibition. Basel, Switzerland: SAMPE, May 1996.

[57] E. C. Greenawald, L. J. Levenberry, N. Qaddoumi, A. McHardy, R. Zoughi, and C. F. Poranski, "Microwave nde of impact damaged fiberglass and elastomer layered composites," in American Institute of Physics Conference Series, vol. 509, no. 1. Montreal, Canada: AIP, July 1999, pp. 1263-1268.

[58] R. Zoughi, J. Lai, and K. Munoz, "A brief review of microwave testing of stratified composite structures: a comparison between plane wave and near field approaches," Materials Evaluation, vol. 60, no. 2, pp. 171-177, February 2002.

[59] S. A. Ramakrishna, "Physics of negative refractive index materials," Reports on Progress in Physics, vol. 68, no. 2, pp. 449-521, February 2005.

[60] B. E. A. Saleh and M. C. Teich, Fundamentals of Photonics. Wiley Series in Pure and Applied Optics, 2nd ed. Hoboken, NJ: John Wiley \& Sons, 2007.

[61] D. R. Smith, J. Gollub, J. J. Mock, W. J. Padilla, and D. Schurig, "Calculation and measurement of bianisotropy in a split ring resonator metamaterial," Journal of Applied Physics, vol. 100, no. 2, pp. 024 507.1-9, July 2006.

[62] J. S. Derov, B. Turchinetz, E. E. Crisman, A. J. Drehman, and R. Wing, "Negative index metamaterial for selective angular separation of microwaves by polarization," in Antennas and Propagation Society International Symposium, IEEE, vol. 4. Monterey, CA: IEEE, June 2004, pp. 3753-3756.

[63] J. D. Wilson and Z. D. Schwartz, "Multifocal flat lens with left-handed metamaterial," Applied Physics Letters, vol. 86, no. 2, pp. $021113.1-3$, January 2005.

[64] F. A. Jenkins and H. E. White, Fundamentals of Optics, Fourth Edition. McGraw-Hill inc. New York, 1976.

[65] M. Bass, E. W. Van Stryland, D. R. Williams, and W. L. Wolfe, Handbook of Optics, 2nd ed. McGraw-Hill, 1995, vol. 2.

[66] J. B. Pendry and S. A. Ramakrishna, "Near-field lenses in two dimensions," Journal of Physics: Condensed Matter, vol. 14, no. 36, pp. 8463-8479, August 2002.

[67] V. A. Podolskiy, N. A. Kuhta, and G. W. Milton, "Optimizing the superlens: Manipulating geometry to enhance the resolution," Applied Physics Letters, vol. 87, no. 23, pp. 231 113.1-3, November 2005.

[68] A. K. Popov and V. M. Shalaev, "Compensating losses in negative-index metamaterials by optical parametric amplification," Optics Letters, vol. 31, no. 14, pp. 2169-2171, July 2006.

[69] D. Shreiber, "Design, development and application of a novel microwave nondestructive evaluation sensor based on metamaterial lens," Ph.D. dissertation, University of Virginia, Charlottesville, VA, 2008.

[70] J. K. Gansel, M. Wegener, S. Burger, and S. Linden, "Gold helix photonic metamaterials: a numerical parameter study," Optics Express, vol. 18, no. 2, pp. 1059-1069, January 2010. 
[71] D. A. McGillivray, R. L. Cravey, K. L. Dudley, and M. C. Gupta, "Metamaterial lens based 2-d microwave imaging system," 2015, unpublished.

[72] D. A. McGillivray, R. L. Cravey, K. L. Dudley, E. Vedeler, and M. C. Gupta, "Polarization properties of a one dimensional metamaterial lens," Microwave and Optical Technology Letters, vol. 56, no. 5, pp. 1218-1222, May 2014.

[73] D. A. McGillivray, R. L. Cravey, K. L. Dudley, E. Vedeler, and M. C. Gupta, "Polarization properties of a 2-d split ring resonator and rod type metamaterial lens," Microwave and Optical Technology Letters, vol. 56, no. 9, pp. 2146-2150, September 2014.

[74] R. K. Amineh, A. Khalatpour, and N. K. Nikolova, "Three-dimensional microwave holographic imaging using co- and cross-polarized data," Antennas and Propagation, IEEE Transactions on, vol. 60, no. 7, pp. 3526-3531, July 2012.

[75] K. M. Yemelyanov, N. Engheta, A. Hoorfar, and J. A. McVay, "Adaptive polarization contrast techniques for through-wall microwave imaging applications," Geoscience and Remote Sensing, IEEE Transactions on, vol. 47, no. 5, pp. 1362-1374, May 2009.

[76] R. Yang, Y. Xie, X. Yang, R. Wang, and B. Chen, "Fundamental modal properties of srr metamaterials and metamaterial based waveguiding structures," Optics Express, vol. 17, no. 8, pp. 6101-6117, April 2009.

[77] I. V. Minin and O. V. Minin, "Concept of near-field millimeter-wave imaging system with a spatial resolution beyond the abbe barrier," in Microwave Conference, 2008 China-Japan Joint. Shanghai, China: IEEE, September 2008, pp. 509-512.

[78] A. K. Iyer and G. V. Eleftheriades, "Free-space imaging beyond the diffraction limit using a veselagopendry transmission-line metamaterial superlens," Antennas and Propagation, IEEE Transactions on, vol. 57, no. 6, pp. 1720-1727, June 2009 .

[79] Y. Lee, S. Zumoberhaus, J. Mosig et al., "Figure-of-merit analysis of resonant particles for construction of practical metamaterials," in Antennas and Propagation, 200\%. EuCAP 200\%. The Second European Conference on. Edinburgh, Scotland: IET, November 2007, pp. 1-4.

[80] H. Chen, L. Ran, J. Huangfu, X. Zhang, K. Chen, T. M. Grzegorczyk, and J. A. Kong, "Left-handed materials composed of only s-shaped resonators," Physical Review E, vol. 70, no. 5, pp. 057605.1-4, November 2004.

[81] H. Chen, L. Ran, J. Huangfu, X. Zhang, K. Chen, T. M. Grzegorczyk, and J. A. Kong, "Negative refraction of a combined double s-shaped metamaterial," Applied Physics Letters, vol. 86, no. 15, pp. 151 909.1-3, April 2005.

[82] M. Kafesaki, I. Tsiapa, N. Katsarakis, T. Koschny, C. M. Soukoulis, and E. N. Economou, "Left-handed metamaterials: The fishnet structure and its variations," Physical Review B, vol. 75, no. 23, pp. 235 114.1-9, June 2007.

[83] K. Aydin, Z. Li, L. Sahin, and E. Ozbay, "Negative phase advance in polarization independent, multi-layer negative-index metamaterials," Optics Express, vol. 16, no. 12, pp. 8835-8844, June 2008.

[84] N.-H. Shen, G. Kenanakis, M. Kafesaki, N. Katsarakis, E. Economou, and C. Soukoulis, "Parametric investigation and analysis of fishnet metamaterials in the microwave regime," Journal of the Optical Society of America B, vol. 26, no. 12, pp. B61-B67, December 2009.

[85] S. Zhang, Y.-S. Park, J. Li, X. Lu, W. Zhang, and X. Zhang, "Negative refractive index in chiral metamaterials," Physical Review Letters, vol. 102, no. 2, pp. 023 901.1-4, January 2009.

[86] N. Kundtz and D. R. Smith, "Extreme-angle broadband metamaterial lens," Nature Materials, vol. 9, no. 2, pp. 129-132, December 2010.

[87] D. R. Smith, J. J. Mock, A. F. Starr, and D. Schurig, "Gradient index metamaterials," Physical Review E, vol. 71, no. 3, pp. $036609.1-6$, March 2005. 
[88] A. Poddubny, I. Iorsh, P. Belov, and Y. Kivshar, "Hyperbolic metamaterials," Nature Photonics, vol. 7, no. 12, pp. 948-957, November 2013.

[89] H. Tao, A. C. Strikwerda, K. Fan, W. J. Padilla, X. Zhang, and R. D. Averitt, "Mems based structurally tunable metamaterials at terahertz frequencies," Journal of Infrared, Millimeter, and Terahertz Waves, vol. 32, no. 5, pp. 580-595, May 2011.

[90] D. Shrekenhamer, C. M. Watts, and W. J. Padilla, "Terahertz single pixel imaging with an optically controlled dynamic spatial light modulator," Optics Express, vol. 21, no. 10, pp. 12 507-12 518, May 2013.

[91] H. Tao, M. A. Brenckle, M. Yang, J. Zhang, M. Liu, S. M. Siebert, R. D. Averitt, M. S. Mannoor, M. C. McAlpine, J. A. Rogers, D. L. Kaplan, and F. G. Omenetto, "Silk-based conformal, adhesive, edible food sensors," Advanced Materials, vol. 24, no. 8, pp. 1067-1072, January 2012.

[92] M. S. Mannoor, H. Tao, J. D. Clayton, A. Sengupta, D. L. Kaplan, R. R. Naik, N. Verma, F. G. Omenetto, and M. C. McAlpine, "Graphene-based wireless bacteria detection on tooth enamel," Nature Communications, vol. 3, pp. 763.1-8, March 2012.

[93] S. J. Park, J. T. Hong, S. Choi, H. S. Kim, W. K. Park, S. T. Han, J. Y. Park, S. Lee, D. S. Kim, and Y. H. Ahn, "Detection of microorganisms using terahertz metamaterials," Scientific Reports, vol. 4, pp. 4988.1-7, May 2014.

[94] J. R. Taylor, An Introduction to Error Analysis, The Study of Uncertainties in Physical Measurements, 2nd ed. Sausalito, CA: University Science Books, 1997. 


\section{Appendix A: Calibration for}

\section{Free-Space Microwave Materials}

\section{Characterization}

The standard Thru Reflect Line calibration procedure is a widely adapted calibration technique for microwave measurements in waveguides and coaxial cables. The Agilent network analyzer offers several calibration schemes: the Full 2 Port (otherwise known as the Short Open Load Transmission (SOLT)), Thru Reflect Line (TRL), Thru Reflect Match (TRM), and Gate Reflect Line (GRL, available as an add-on from Agilent). These calibration procedures are very robust and promise very accurate error correction. However, each one of these calibration techniques offer their own set of drawbacks pertaining to free space measurements. These drawbacks come from the constraints the calibration techniques impose on the free space calibration procedures and reference standards. With any of these calibration techniques millimeter or better precision in positioning of the horns and reference standards is required, thus eliminating complexities is highly desirable. The typical calibration standards for microwave error corrections are: short, open, load or match, thru, line, and isolation. These come from the equivalent circuit theory of transmission lines, and can be adapted to free space as follows:

1. The short is an ideal reflector with a $180^{\circ}$ phase change at the reference interface; in free space this translates to a clean, flat metal sheet of negligible (compared to the wavelength) thickness.

2. The open is also an ideal reflector, with a $90^{\circ}$ phase change. This is realized by increasing the distance between the source and the reference plane by an additional $\lambda / 4$. 
3. The load and match standard are realized for free space as an absorber with better than $-20 \mathrm{~dB}$ absorption.

4. The thru standard is a free path transmission measurement of the horn to horn distance.

5. The line standard is also a free path measurement, with an additional lambda/4 in separation distance between the source and receiver.

6. The isolation standard can be realized by isolating the horn antennas from each other, either by use of a very good load or also by a reflection plate, in order to measure the indirect signal sources.

In the case of the full 2 port calibration, the technique calls for short, open, load and thru reference standards. The TRL calibration requires a thru, short and line standard; the TRM a thru, short and match. The GRL calibration is different in the sense that it relies on a short and a line standard as well as time domain gating.

In literature, the most commonly adapted technique is the TRL calibration due to its easily realizable reference standards, which require only one offset movement. Agilent has developed the GRL calibration as a way to simplify the TRL calibration, requiring no movement in the experiment setup. However, this calibration technique cannot be applied to metamaterials due to the short time domain gating window applied during the calibration. Metamaterials have a uncharacteristically long electrical length, which the GRL calibration cannot account for. Therefore, the TRL calibration was chosen.

The semi anechoic measurement environment at NASA Langley's Electromagnetics and Sensors Branch consisted of cone-type absorber on a front and back wall, floor and a sample stage treated with absorber on both sides (see fig. 3.8). The AEL 2 - $18 \mathrm{GHz}$ horn antennas were connected via semi-rigid phase stable cables, so as not to introduce noise by cable movement, to the Agilent PNA - E network analyzer. The horn source antenna was mounted on an axial rotational stage (rotating about the axis of microwave propagation) as well as on a micrometer stage. The receive horn was also mounted on a rotation stage. Both horns were 
situated at the far-field condition of $2 D^{2} / \lambda$ away from the reference plane of the experiment setup. The calibration standards were defined for free-space in the network analyzer as in table A.1.

$$
\text { LineDelayTime }=\frac{1}{4 \sqrt{F_{s} F_{e}}}
$$

Where $F_{s}$ is the start frequency and $F_{e}$ the end frequency of the experiment.

Table A.1: The reference standard definitions were entered into the network analyzer as follows

\begin{tabular}{|l|ccc|}
\hline Standard & Thru & Reflect & Line \\
\hline Frequency Min $(\mathrm{GHz})$ & 0 & 0 & 0 \\
Frequency Max $(\mathrm{GHz})$ & 999000 & 999000 & 999000 \\
$\mathrm{C} 0 \mathrm{~F}\left(\times 10^{-15}\right)$ & N/A & 0 & N/A \\
$\mathrm{C} 1 \mathrm{~F}\left(\times 10^{-27}\right) / \mathrm{Hz}$ & N/A & 0 & N/A \\
$\mathrm{C} 2 \mathrm{~F}\left(\times 10^{-36}\right) / \mathrm{Hz}$ & N/A & 0 & N/A \\
$\mathrm{C} 3 \mathrm{~F}\left(\times 10^{-45}\right) / \mathrm{Hz}$ & N/A & 0 & N/A \\
Delay Time $\left(10^{-12} s\right)$ & 0 & 0 & Line Delay Time \\
Loss $(G \Omega / s)$ & 0 & 0 & 0 \\
$\mathrm{Z} 0(\Omega)$ & 377 & 377 & 377 \\
\hline
\end{tabular}

For the calibration procedure it is crucial to ensure that the reference planes of the reflection standard are the same size as the reference planes of the thru and line standard. Due to the nature of testing thick materials (thicker than $\lambda / 4$ ) TRL calibration needs post calibration corrections in order to coincide the reference plane of the thru and line standard with the reference plane of the reflect standard.

Reflect calibration standard was realized by attaching adhesive aluminum foil to the material under test's (MUT) front and back surface. This ensured that the reflect standard was the same size as the MUT, and that the reflection planes were parallel with the front and back surface of the MUT. Furthermore, this fixed the front and back surfaces of the MUT as the reference plane. The thru standard was a horn to horn transmission measurement with an empty aperture. The line standard was measured by moving the source horn on the 
micrometer stage by the LineDelayLength $=c \times$ LineDelayTime, where $\mathrm{c}$ is the speed of light.

The calibration procedure started with performing a SOLT calibration to the ends of the cables. The cables were then subsequently hooked up to the horn antennas. The reflection standard was then inserted into the aperture and $S_{11}$ and $S_{22}$ parameters were measured in time domain. The reflection standard was removed and the measurement was repeated. By observing a change in reflection intensity vs time, the reflection-plane and reflection time can be identified for $S_{11}$ and $S_{22}$. Subsequently the source horn was moved in order to match the time it takes for the reflection to occur for both $S_{11}$ and $S_{22}$. This procedure ensured that the source and receive horn were placed the same distance from the reference planes.

Once the source and reflect horn were properly aligned, the TRL calibration procedure was started. The TRL calibration was referenced to the reflect. The measurements were taking from $3 \mathrm{GHz}$ to $6 \mathrm{GHz}$ with 2001 data points. The order in which the standards were measured did not matter.

Post calibration errors were corrected by measuring an empty thru and performing a baseline subtraction as well as adding an electrical delay equal to the thickness of the MUT $\left(\right.$ Delay $\left._{M U T}\right)$ to the thru measurement. Delay $y_{M U T}=z_{M U T} / c$, where $z_{M U T}$ is the thickness of the MUT and $\mathrm{c}$ is the speed of light.

This baseline subtraction ensured that the transmission and reflection reference planes were the same. This was confirmed by measuring the transmission and reflection time in the time-domain. Subsequently, the MUT was inserted into the sample holder and S-parameter measurements were performed. The fig. A.1-A.3 show the measurement uncertainties of the reference standards (reflection standard see fig. A.1, thru standard see fig. A.2, and line standard see fig. A.3); overall the measurements were within $\pm 0.78 \mathrm{~dB}$ in magnitude and $\pm 3.5^{\circ}$ degrees in phase as compared to the ideal. It is possible to further reduce post-calibration errors by using time-domain gating technique. With gating, the overall measurement uncertainties were $\pm 0.62 \mathrm{~dB}$ in magnitude, and $\pm 1.92^{\circ}$ degrees in phase. 
However, due to their long electrical delay, metamaterials will have different time-gates for reflection and transmission. Therefore for reflection measurements on the MTM time gates are chosen to be much shorter ( 2 ns gating window) than those for transmission (15 ns gating window). The gate shape did not have an appreciable effect in the measurement and was left at the default (wide) setting.

(a) Reflect Standard: Reflection Magnitude

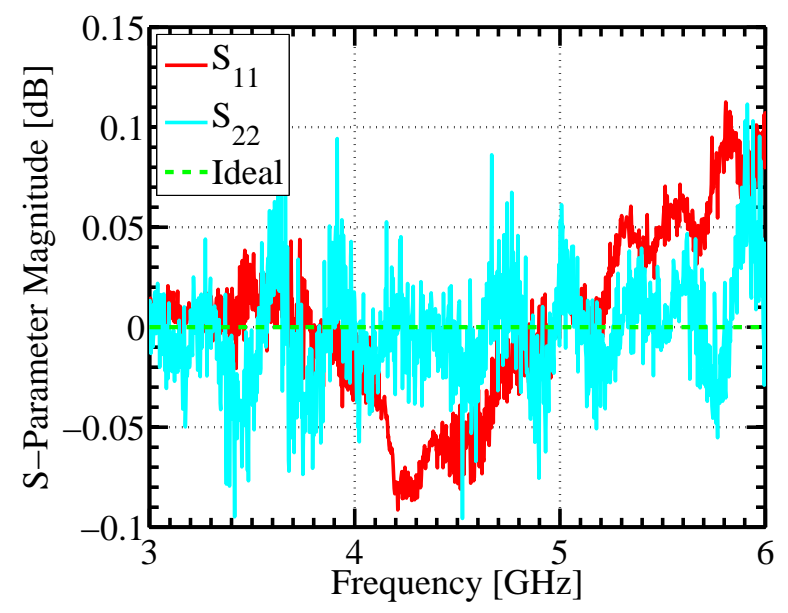

(c) Reflect Standard: Reflection Phase

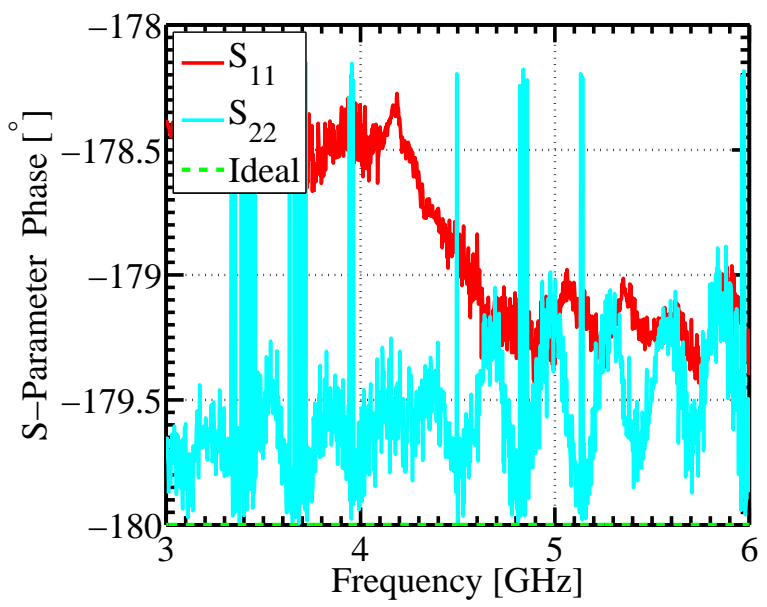

(b) Reflect Standard: Transmission Magnitude

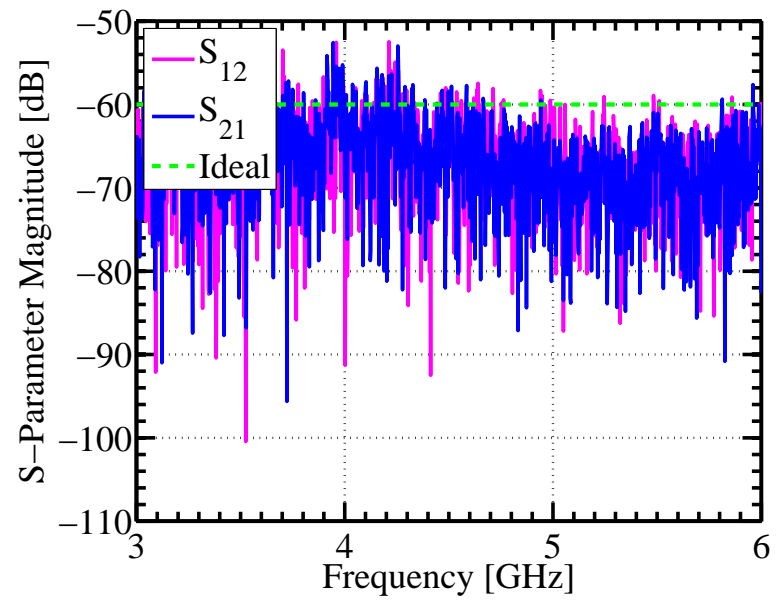

(d) Reflect Standard: Transmission Phase

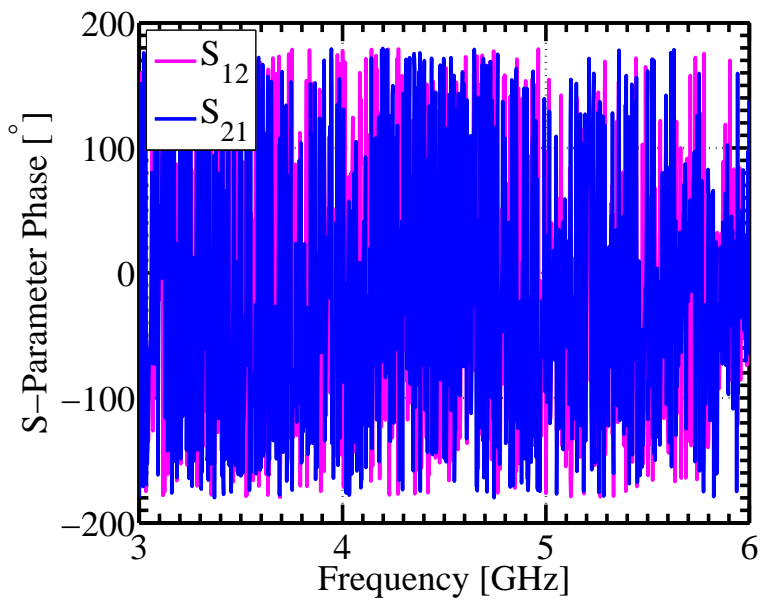

Figure A.1: Measurement uncertainties of the reflect standard. Error in Reflection magnitude is $\pm 0.12 \mathrm{~dB}$. Reflection phase error is $\pm 1.85^{\circ}$. Transmission magnitude is the isolation between the horns and is very good at less than $-50 \mathrm{~dB}$. Transmitted phase does not give accurate numbers since the measurement is in the noise of the measurement environment. 
(a) Thru Standard: Reflection Magnitude

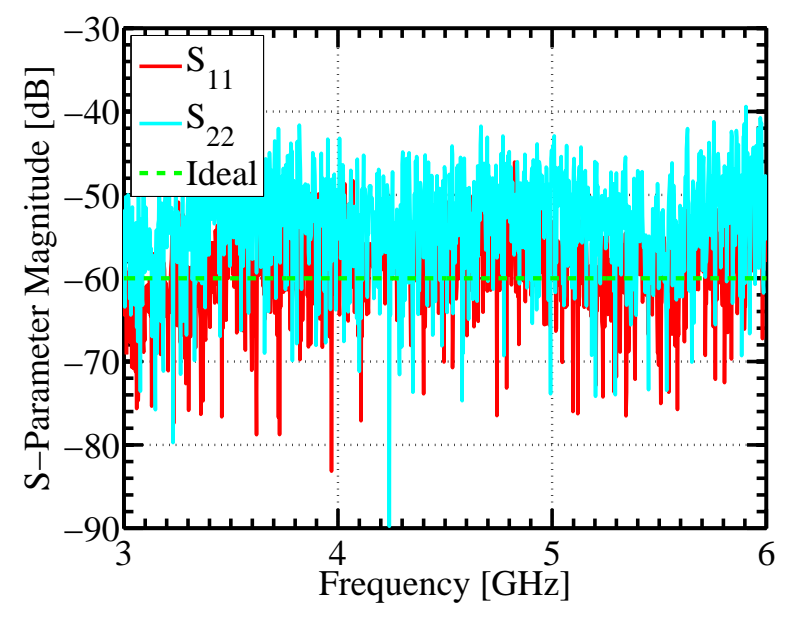

(c) Thru Standard: Reflection Phase

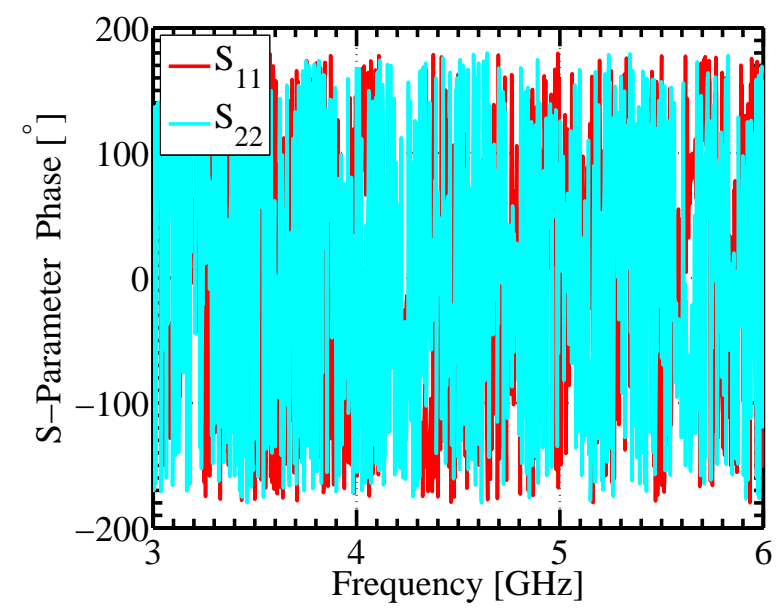

(b) Thru Standard: Transmission Magnitude

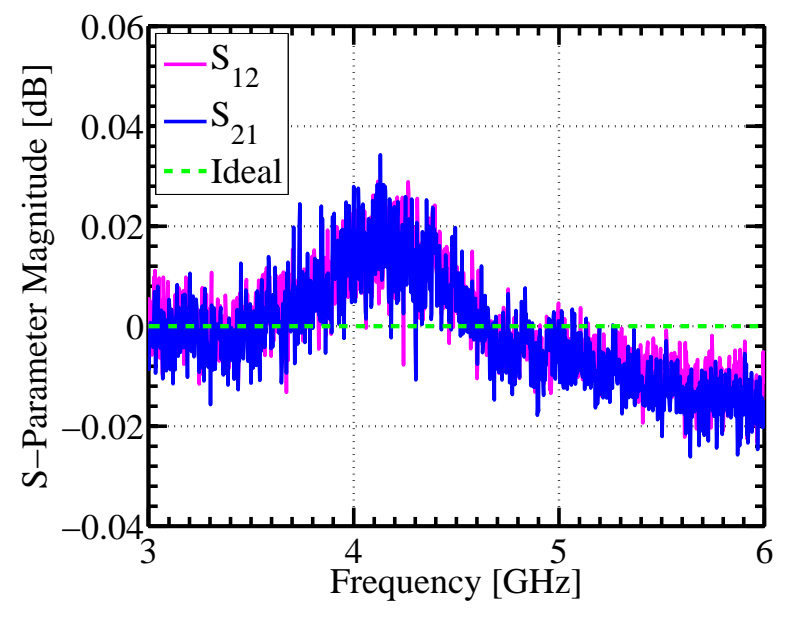

(d) Thru Standard: Transmission Phase

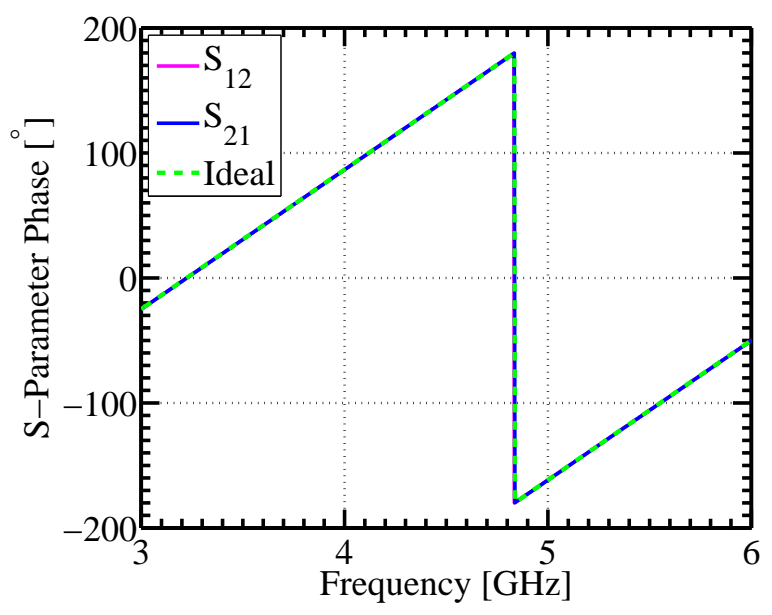

Figure A.2: Measurement uncertainties of the thru standard. Reflection magnitude was less than $-40 \mathrm{~dB}$, indicating that the back-reflection in the test range was very good. Reflection Phase is in the noise of the environment. Error in transmission magnitude is $\pm 0.03 \mathrm{~dB}$. Transmission Phase is in good agreement with the ideal phase with an error of $\pm 0.35^{\circ}$ degrees. 
(a) Line Standard: Reflection Magnitude

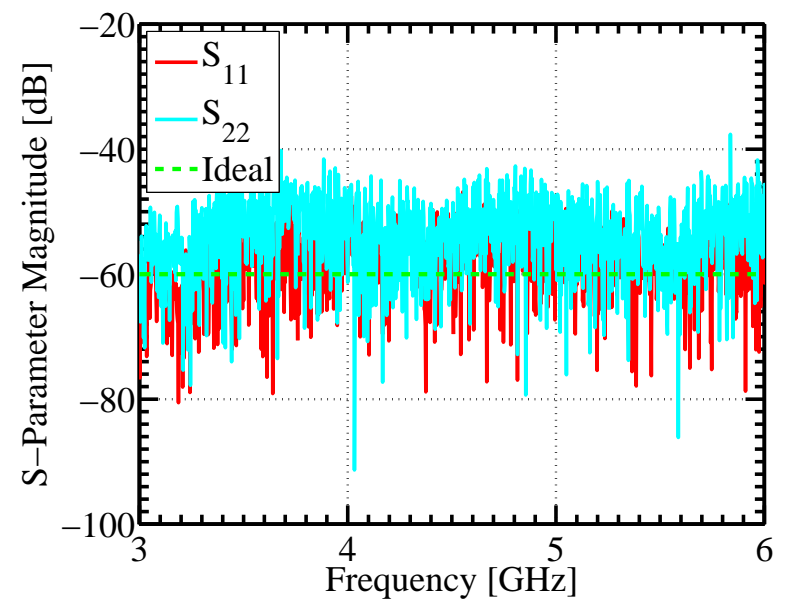

(c) Line Standard: Reflection Phase

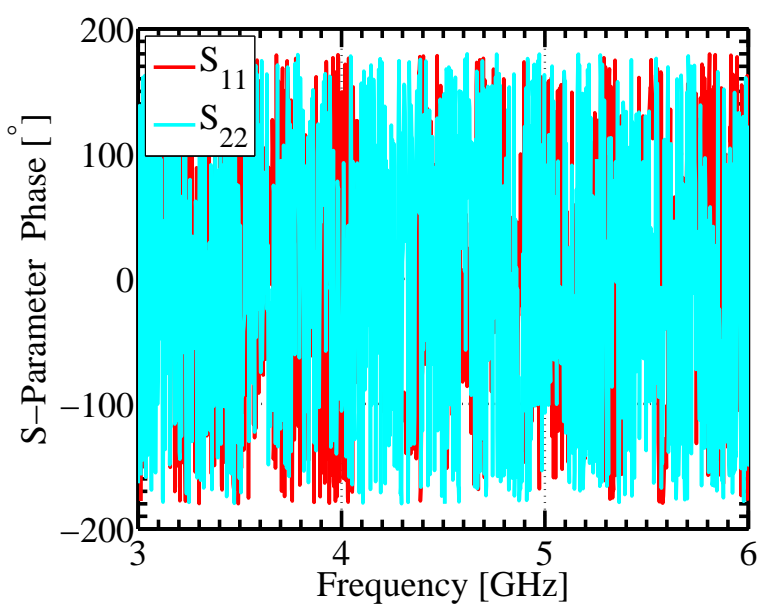

(b) Line Standard: Transmission Magnitude

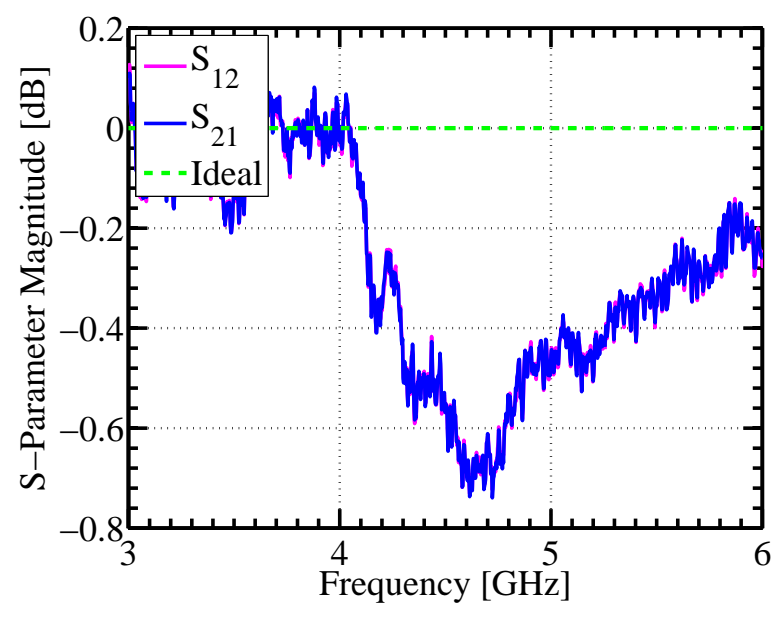

(d) Line Standard: Transmission Phase

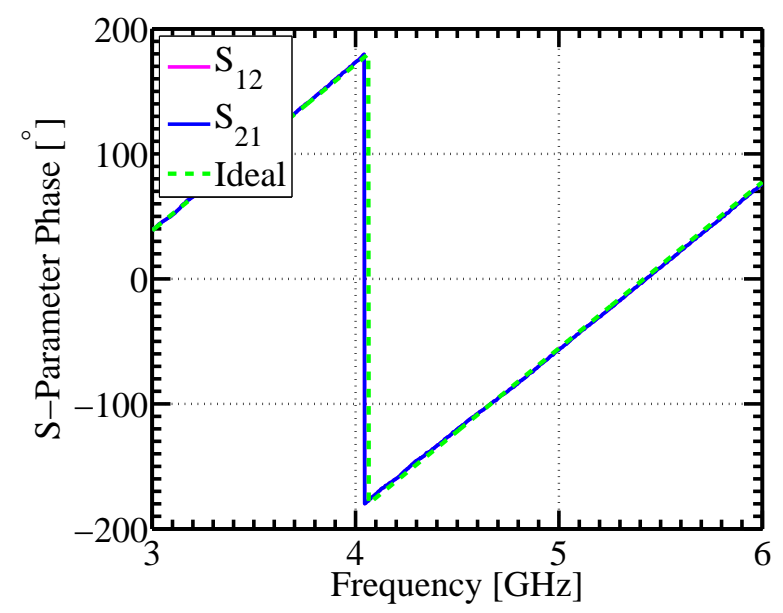

Figure A.3: Measurement uncertainties of the line standard. Reflection magnitude was less than $-40 \mathrm{~dB}$, indicating that the back-reflection in the test range was very good. Reflection phase is in the noise of the environment. In reference to a coaxial measurement environment, the ideal transmission magnitude would be 0 . The error in transmission magnitude is $\pm 0.78 \mathrm{~dB}$. Error in transmitted phase is $\pm 3.5^{\circ}$ degrees. 
The calibration standards were used to extract the measurement uncertainty in the EM properties characterization experiment. The uncertainties were calculated for magnitude and phase and applied to the S-parameter measurements. Measurement errors were then estimated through error propagation during the extraction of the index of refraction (eq. 4.1 through eq. 4.3). Error propagation was carried out using the techniques described in [94]. Fig. A.4 shows the errors associated with the measurement of the 2-D LFL for the transmission and reflection in magnitude (fig. A.4a), phase (fig. A.4b) and extracted index of refraction (fig. A.4c). The maximum error in magnitude for transmission and reflection were $\pm 6.8 \%$ and $\pm 0.8 \%$, respectively. The maximum phase measurement errors were $\pm 0.3 \%$ for reflection and $\pm 1.7 \%$ for transmission. Error propagations for index of refraction show uncertainties of up to $\pm 13 \%$ for frequencies below $4 \mathrm{GHz}$ and uncertainties up to $\pm 31 \%$ for frequencies above $4 \mathrm{GHz}$. The index of refraction is very susceptible to very small errors in S-parameter measurements. At the 2-D LFL resonance frequency of $3.658 \mathrm{GHz}$ the index of refraction has a measurement uncertainty of $\pm 6.4 \%$ which amounts to $n^{\prime}=-1.11 \pm 0.07$ and $\kappa=0.46 \pm 0.03$. Similar measurement uncertainty is expected for the characterization of the HFL. 
(a) Magnitude

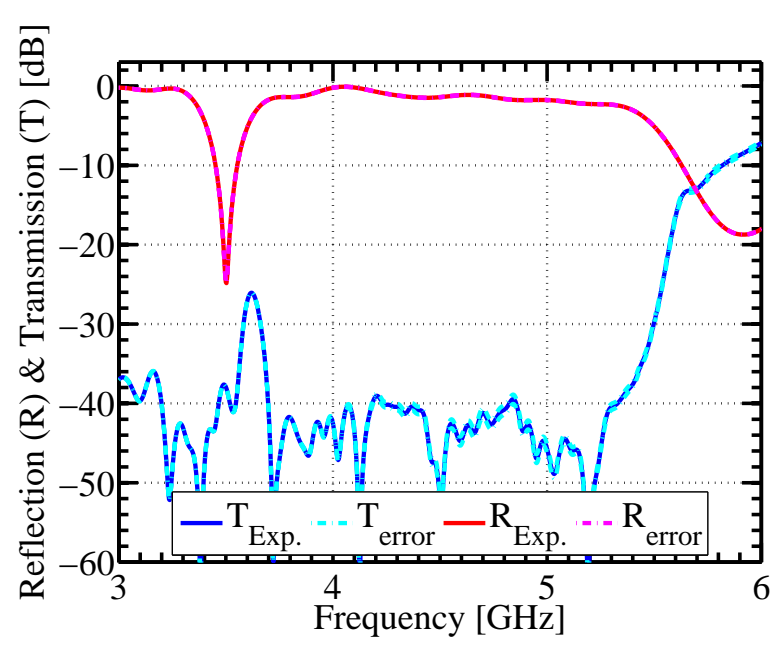

(b) Phase

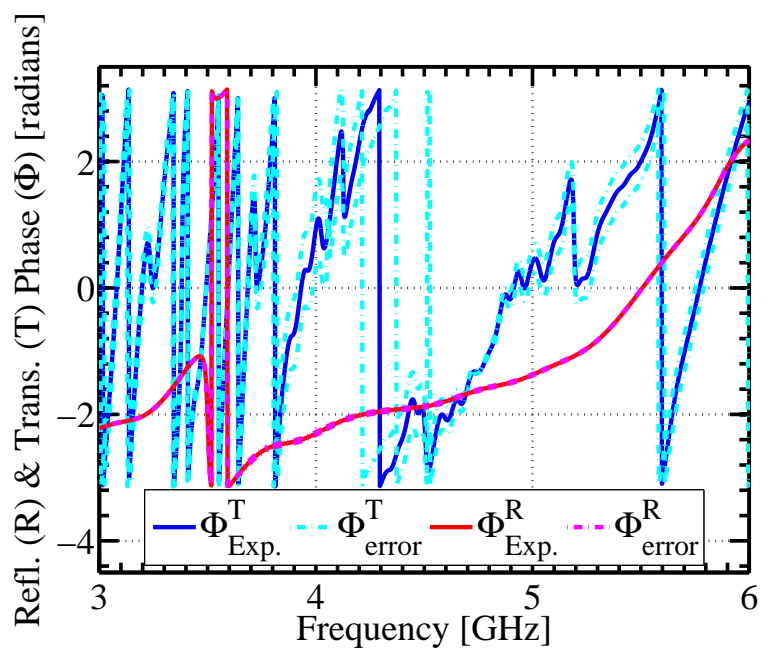

(c) Index of refraction

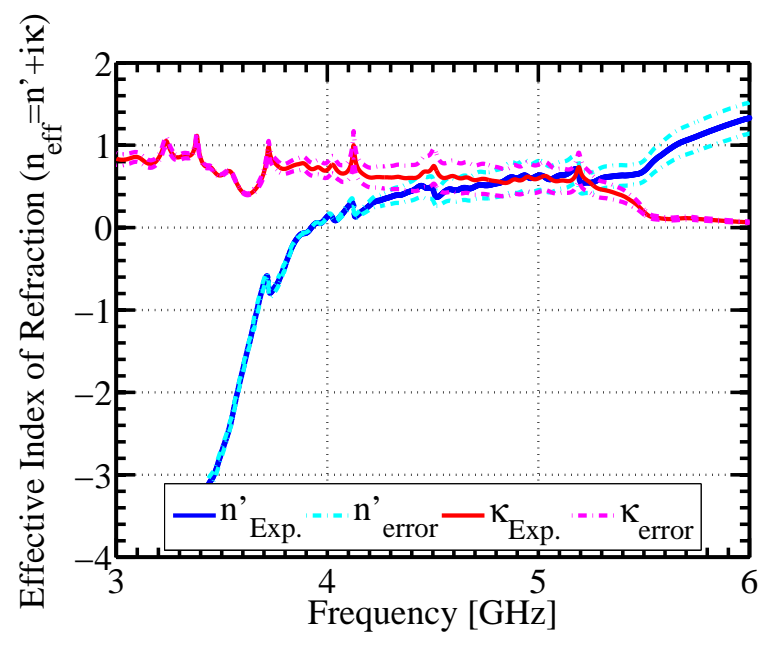

Figure A.4: Measurement uncertainties of the EM properties for the 2-D LFL. (a) errors in transmission and reflection magnitude were very small. (b) errors in phase were small except for higher frequency data. (c) errors propagation for the in the index of refraction show very small uncertainty for the negative index region, however grow above $4 \mathrm{GHz}$. 


\section{Appendix B: Detector Calibration}

Detector calibration was straight forward for the individual detectors. A detector was linked to one of the ports of the network analyzer. The network analyzer was set to continuous wave at the imaging frequency of the MTM lens. The network analyzer's internal power was then swept from $-60 \mathrm{dBm}$ to $0 \mathrm{dBm}$ and the detected voltage was recorded. The power sweep was cycled three consecutive times.

A second system measurement was performed, where the antenna and detector pair was placed into free space and illuminated in the far-field with a source horn antenna. The source horn was driven by a $+30 \mathrm{dBm}$ amplifier and the network analyzer. The source was set to continuous wave and the source power was then swept from $-60 \mathrm{dBm}$ to $0 \mathrm{dBm}$.

The combination of both measurements formed the basis for the calibration curve. The first measurement provided for an ideal voltage out versus power in measurement, while the second measurement provided for any voltage offsets due to the free-space measurement environment. The primary source for voltage offsets was traced to the grounding mechanism of the detector. When attached to the network analyzer, the detector shared the same ground as the network analyzer, that is the building ground. In free-space the detector was grounded to the data acquisition system, i.e. the laptop.

Fig. B.1 provides the calibration curve for detector 1; the same calibration procedure was carried out for all of the detectors. It is of note that the calibration curves were not all identical, as each diode detector proved to be susceptible to small variations in load resistance. The overall imaging system was calibrated by using this calibration procedure in combination with referencing each image to a blank sample image. 
(a) Detector 1

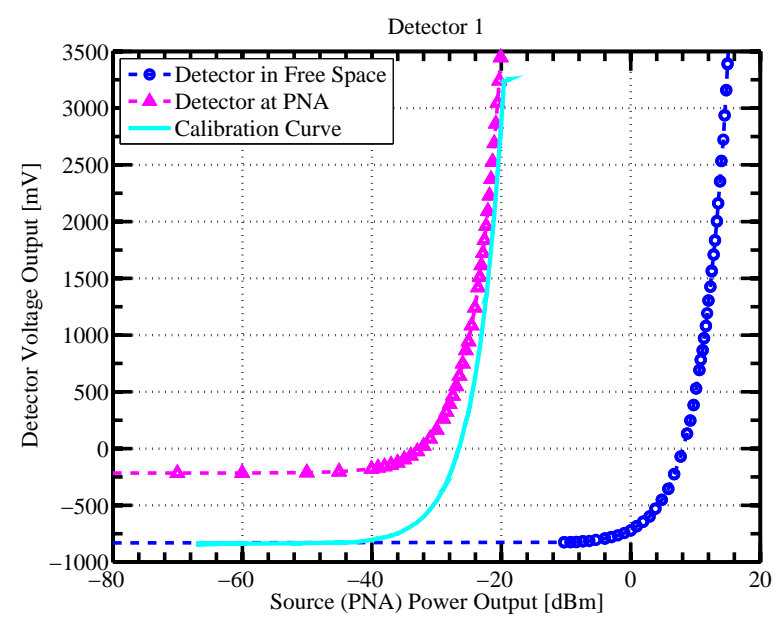

(b) Multi Detectors

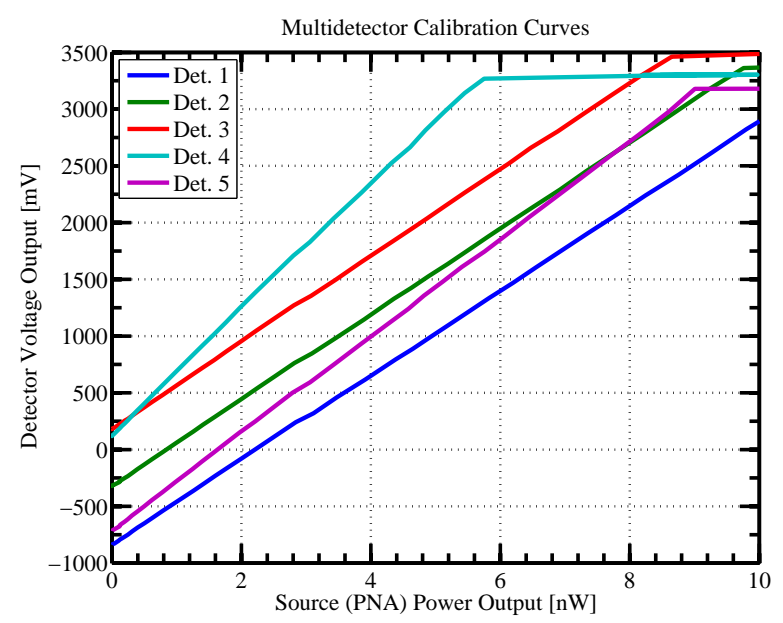

Figure B.1: Detector calibration curves as a function of power sweep versus detected voltage are presented. (a) the calibration curve is extracted by offsetting the curve fit data from the precision network analyzer (PNA) measurement by the voltage offset of the free-space measurement. (b) the various detector calibration curves are presented. 